\section{Breast Pathology}

101 Overexpression of FoxO3a Is Associated with Lymph Node Metastasis and Poor Disease-Free Survival in Triple-Negative Breast Cancers

Rehman Abdul, Yumin Chung, Hyein Ahn, Jongmin Sim, Min Sung Chung, Kiseok Jang. Hanyang University College of Medicine, Seoul, Republic of Korea.

Background: Triple negative breast cancer (TNBC) is the most aggressive type of breast cancer, whichis a heterogeneous group and has no proven molecular target. FoxO3a, a transcription factor, is involved in wide spectrum of biological processes, including cell cycle progression, proliferation, DNA damage repair, and apoptosis.Recently, accumulating evidences suggests that FoxO3a act as a tumor suppressorin variety of human cancers. However,the previous studies usingTNBC cell lines have shown controversial results. The aim of this study was to investigate the clinicopathological significance and role of FoxO3a in the progression of TNBC.

Design: Tissue microarrays consisting of 124 cases of TNBC were studied for FoxO3a expression by immunohistochemistry and interpreted by semi-quantitative scoring system. The FoxO3a expression correlated with various clinicopathological parameters, including patient's survival. Furthermore, cultured TNBC cell lines (MDA-MB-231, MDA-MB-468, BT20) were assessed for FoxO3a expression by western blot. MDAMB-468 breast cancer cell line was transiently transfected with exogenous siRNA, which wasspecific for FoxO3a. The effect of siRNA treatment on cell proliferation and migration ability was analyzed by MTT assay and wound healing assay, respectively. Results: Overexpression of FoxO3a was correlated with adverse clinicopathological features, such as lymph node metastasis $\left(\mathrm{p}=0.021, \chi^{2}\right.$-test) andperineural invasion $\left(\mathrm{p}=0.013, \chi^{2}\right.$-test). High FoxO3a expression is significantly correlated with poor disease-free survival ( $\mathrm{p}=0.015$, log-rank test). The tumors with high FoxO3a expression had higher Ki-67 proliferation index ( $\mathrm{p}=0.041$, $\mathrm{t}$-test). Among TNBC cell lines (MDAMB-231, MDA-MA-468, and BT20), MDA-MA-468 had expression of FoxO3a by western blot analysis. There was no significant change in cell proliferation by siRNAmediated FoxO3a down-regulation in vitro. However, the inhibition of FoxO3arevealed to decrease cellular migration compared with negative controlin wound healing assay. Conclusions: FOXO3a may have a potential role of promoting invasion and proliferation of TNBC cells, and may be useful as prognostic biomarker and a newpotential therapeutic targetin TNBC.

102 Role of Epithelial-to Mesenchymal Transition Markers in Metaplastic Breast Cancer with Coexistent Ductal Carcinoma In-Situ Eman Abdulfatah, Rahman Chaudhry, Vishakha Pardeshi, Muhammad K Alsafadi, Sherif Shazly, Rouba Ali-Fehmi, Sudeshna Bandyopadhyay. WSU, Detroit, MI; Mayo, Rochester, MN.

Background: Metaplastic breast carcinomas (MBC) are rare heterogeneous group of tumors that are part of the basal carcinoma spectrum, displaying a basal/myoepithelial and epithelial-to-mesenchymal (EMT) molecular makeup. However, the clinical significance of EMT in MBC has not been fully investigated. In this study, we evaluated the expression of EMT and cancer stem cell markers (CSC) in ductal carcinoma in-situ (DCIS) and invasive component and correlated with phenotypic features and clinical outcome.

Design: Retrospective review of MBC $(n=68)$ was conducted. Cinicopathologic parameters were analyzed (Table 1). Slides were reviewed for DCIS associated metaplastic features. IHC of CSC (CD44, CD24 and ALDH1) and EMT markers (vimentin, SNAIL, SLUG and loss of E-cadherin) expression was performed on whole sections of a subset $(\mathrm{n}=20)$. Each marker was evaluated per established criteria and their expression was correlated with overall survival (OS) using Kaplan-Meier anaylsis. Results: All tumors were triple negative. Axillary lymph node metastasis was identified in 22 cases (32\%). 27 cases showed co-existent DCIS, of which 14 cases $(52 \%)$ showed metaplastic features; the highest being squamous (11), followed by rhabdoid (2) and chondroid (1). EMT and CSC markers showed variable levels of expression among DCIS, glandular and metaplastic components (MC) (Table 2). Of all markers evaluated, high SNAIL expression correlated with decreased OS $(\mathrm{p}=0.03)$.

\begin{tabular}{|l|l|}
\hline Clincopathologic parameters & $\mathbf{n}=\mathbf{6 8}(\mathbf{\%})$ \\
\hline Predominant MC & \\
\hline Squamous & $31(45.5)$ \\
\hline Spindle & $15(22)$ \\
\hline Chondroid & $9(13)$ \\
\hline Osseous & $4(6)$ \\
\hline Pseudoangiomatous & $6(9)$ \\
\hline Mixed & $2(3)$ \\
\hline HG sarcomatoid & $1(1.5)$ \\
\hline DCIS with metaplastic features & $14(52)$ \\
\hline Squamous & $11(78.5)$ \\
\hline Chondroid & $2(14)$ \\
\hline Rhabdoid & $1(7.5)$ \\
\hline None & $13(48)$ \\
\hline Grade & \\
\hline 1 & $1(1.5)$ \\
\hline 2 & $3(4.5)$ \\
\hline 3 & $64(94)$ \\
\hline Stage & \\
\hline I-II & $46(68)$ \\
\hline III-IV & $22(32)$ \\
\hline Recurrence & \\
\hline Yes & $12(17.5)$ \\
\hline No & $56(82.5)$ \\
\hline & \\
\hline
\end{tabular}

\begin{tabular}{|l|l|l|l|l|l|l|l|}
\hline & CD44+ & CD24- & ALDH1 & SNAIL & SLUG & Vimentin & E-cadherin \\
\hline $\begin{array}{l}\text { DCIS, } \\
\mathbf{n = 1 4 ( \% )}\end{array}$ & $8(73)$ & $8(73)$ & $4(29)$ & $11(79)$ & $6(43)$ & $6(43)$ & $6(43)$ \\
\hline $\begin{array}{l}\text { Glandular } \\
\text { component, } \\
\text { n=20(\%) }\end{array}$ & $8(40)$ & $8(40)$ & $3(15)$ & $11(55)$ & $8(40)$ & $11(55)$ & $5(25)$ \\
\hline $\begin{array}{l}\text { Metaplastic } \\
\text { component, } \\
\text { n=20(\%) }\end{array}$ & $20(100)$ & $20(100)$ & $13(65)$ & $17(85)$ & $14(70)$ & $16(80)$ & $17(85)$ \\
\hline
\end{tabular}

Conclusions: DCIS associated with MBC may show metaplastic features and express CSC and EMT markers. In our study, high SNAIL expression was associated with poor $\mathrm{OS}$, suggesting its role as a potential prognostic marker.

103 Histologic Characteristics of Breast Carcinomas with Genetic Variants of Uncertain Significance:

Ahmed Abdulrahman, Rebecca Heintzelman, Melanie Corbman, Fernando Garcia. Drexel University College of Medicine, Philadelphia, PA; Cancer Treatment Centers of America at Eastern Regional Medical Center, Philadelphia, PA.

Background: Starting in 2013, genetic testing laboratories incorporated additional genes in the breast cancer molecular testing panel, resulting in detection of new mutations in approximately $40 \%$ of cases. When there is insufficient data to determine if the variant causes increased cancer risk, these have been reported as variants of uncertain significance (VUS). Histopathologic characteristics of VUS breast carcinomas (BCs) have not been previously well-described. Our purpose is to describe common pathologic features in cases with similar VUS genes.

Design: In total, 19 VUS BCs were retrospectively identified and IRB approved from the Genetic Counselor's office, consisting of eighteen female patients and one male patient. Ages ranged from 34 to 71 with a mean age of 53. Representative de-identified $\mathrm{H} \& \mathrm{E}$ tumor sections were reviewed by three of the authors along with the reported CAP tumor characteristics and prognostic markers. In addition, the reported VUS germline mutations, peritumoral inflammation and peritumoral fibrosis were described.

Results: Eight of the 19 cases were VUS with variants in the ATM gene. Three were in CHEK2, two in each of RAD51D, NBN, MUTYH, MLH1, BRCA2, CDKN2A, and one in each of MSH2, MSH6, PMS2, STK11, TP53, CDH1, BRCA1 and APC genes. The cases were grouped for histologic comparison into ATM (8) and non-ATM (11). ATM $\mathrm{BC}$ cases shared similar histologic characteristics when compared to variants in other genes. However, molecular subtyping assessed by immunohistochemistry was different.

\begin{tabular}{|l|l|l|}
\hline Histologic Characteristics & ATM gene VUS (8/19) & Non-ATM gene VUS (11/19) \\
\hline Peritumoral Inflammation & Absent & Present \\
\hline Peritumoral Fibrosis & Prominent & Variable \\
\hline Cytology & $\begin{array}{l}\text { Nuclear Grade 2 } \\
\text { Abundant eosinophilic } \\
\text { cytoplasm }\end{array}$ & $\begin{array}{l}\text { Nuclear Grade 3 } \\
\text { Scant to moderate cytoplasm }\end{array}$ \\
\hline $\begin{array}{l}\text { Molecular Subtypes (in } \\
\text { decreasing frequency) }\end{array}$ & $\begin{array}{l}\text { Luminal A, B and Her2 (A is 4 4 } \\
\text { cases, B is 3 and Her2 is 1) }\end{array}$ & $\begin{array}{l}\text { Luminal B, triple negative and } \\
\text { A (B is 6 cases, triple negative } \\
\text { is 3 and A is 2) }\end{array}$ \\
\hline
\end{tabular}

Conclusions: 1. VUS BCs with ATM gene mutations shared similar histologic characteristics with lack of peritumoral inflammation and marked peritumoral fibrosis 2. VUS BCs with BRCA2 gene mutations shared high histologic grade with anaplasia 3. VUS BCs with MLH1 gene mutations were of pleomorphic lobular type

4. Further study is required to better assess the association of germline gene mutations with peritumoral inflammation and fibrosis 
104 Epithelial-Mesenchymal Transition and Cancer Stem Cells Interactions in Phyllodes Tumours: Clinical Relevance and Prognostic Significance

Syed Salahuddin Ahmed, Jabed Iqbal, Aye Aye Thike, Jeffry Chun Tatt Lim, Puay Hoon Tan. Singapore General Hospital, Singapore, Singapore.

Background: Phyllodes tumours (PTs) are rare fibroepithelial neoplasms of the breast and account for approximately $1 \%$ of all breast tumours. A constellation of histological features such as stromal hypercellularity, stromal cell atypia, mitoses, stromal overgrowth and tumour borders, further classify PTs as benign, borderline and malignant. Histological assessment may not always translate into tumor behaviour, posing challenges in clinical management.

Epithelial-mesenchymal transition (EMT) is an important process during embryogenesis. In tumorigenesis, dysregulation of EMT is postulated to cause loss of cell polarity, decrease intercellular adhesion, increase motility and invasiveness, promoting tumor progression. Similarly it is hypothesized that cancer stem cells (CSCs) are associated with tumor growth, chemoresistance, and recurrence. We aim to evaluate immunohistochemical expression of EMT and CSCs markers in PTs and correlate the expression of these markers with clinicopathological parameters and outcomes. Design: Immunohistochemistry was performed on 360 PTs using tissue microarray sections. The expression of molecules related to EMT (Ezrin and HMGA2) and CSCs markers (EZH2) was assessed in stromal cells of tumors. Disease free survival (DFS) and overall survival (OS) were defined as time from diagnosis to death/date of last follow up. Biomarker expression was correlated with clinicopathological parameters and outcomes. Results: Of the 360 cases, 241 (67\%) tumors were benign, 87 (24.2\%) borderline and $32(8.9 \%)$ malignant. Patient age ranged from 15 to 79 years (mean and median 43 years), tumour size ranged from 8 to $250 \mathrm{~mm}$ (mean 51, median 58). Stromal expression of Ezrin, HMGA2 and EZH2 was observed in 16\%, $16 \%$ and $20 \%$ of tumors respectively. Statistically significant correlation $(p<0.001)$ was observed with expression of Ezrin, EZH2 and HMGA2 in relation to the tumor grade, stromal cell atypia, stromal overgrowth, increased stromal mitoses and microscopic permeative borders. Patients whose tumors expressed EZH2 disclosed early recurrence $(\mathrm{p}=0.012)$ and poorer $\mathrm{OS}(\mathrm{p}=0.009)$

Conclusions: Determination of EMT and CSCs markers provides significant biological insights into breast PTs. These markers may promote new therapeutic paradigms in management, especially for malignant tumors.

\section{Gene Expression Analysis in Estrogen Receptor Subsets Defined} by Immunohistochemistry in Breast Invasive Duct Carcinoma

Di Ai, Bing Leng, Kimberly L Walker, Shelby Hendrickson, Ada Werlang-Perurena, Arundhati Rao. Baylor Scott \& White Memorial Hospital, Temple, TX; MD Anderson Cancer Center, Houston, TX.

Background: Estrogen Receptor (ER) positivity by immunohistochemistry (IHC) in breast cancer was lowered according to $\mathrm{ASCO} / \mathrm{CAP}$ guidelines from $10 \%$ to $1 \%$ of tumor cells in 2010. Some studies have reported that there was no significant difference in overall survival and recurrent-free survival with negative ER IHC, ER IHC 1-5\% or ER IHC 6-10\% positive subsets, suggesting that the biological behavior of ER 1-10\% groups is similar to those in ER negative group. We hypothesize that gene expression analysis of ER pathway related genes may provide information regarding relatedness and identify other predictors of outcome.

Design: Total mRNA was extracted from breast cancer specimens (Qiagen, MD). mRNA expressions of 192 breast cancer-related genes in ER negative, ER 1-10\% and ER $>50 \%$ were quantitated using the nCounter Analysis System (NanoString, WA) and analyzed using nSolver Analysis Software (V2.5). Tissue microarray (TMA) of corresponding tumor sections was subjected to immunohistochemistry to confirm subsets with differential expression. Each IHC staining was assessed: a. percentage of positive cells ( 0 : negative, $1: 1-10 \%$ positive, $2: 10-50 \%$ positive, $3:>50 \%$ positive) and $b$. staining intensity $(0$ : negative, $1+, 2+$ and $3+)$. T-test was used for statistical analysis and $\mathrm{p}$ value of 0.05 is considered significantly different

Results: 46 breast cancer specimens with 3-5 years follow up and treatment data, including $27 \mathrm{ER}$ IHC negative, $12 \mathrm{ER}$ IHC $1-10 \%$ positive and $7 \mathrm{ER}$ IHC $>50 \%$ positive were selected, reviewed and confirmed by image analysis. Heatmap analysis identified 151 of $192(78.6 \%)$ genes in the low ER group positively correlated with those in ER negative group and negatively correlated with those in ER IHC $>50 \%$ group. Three proteins, progesterone receptor (PR), GATA 3 and serpin 1 had the highest negative correlation and correlated with IHC. GATA-3 IHC scores in ER negative, ER 1-10\% and ER $>50 \%$ are: $1.44,2.08$ and 7.29 , respectively. P value of ER negative vs ER $1-10 \%$; ER negative vs ER $>50 \%$ and ER negative vs ER $>50 \%$ were: $0.22,0.0013$ and 00023 , respectively.

Conclusions: mRNA expression of breast cancer-ER related genes in different ER IHC groups can be robustly profiled and the molecular profile (both mRNA and protein) in ER low positive group is similar to the ER negative group, rather than the ER $>50$ group. Addition of GATA 3 and PR statusto the low positive ER group may help with better classification for therapy.

106 Tumor Infiltrating Lymphocytes (TIL) in Predicting Neoadjuvant Therapy Response and Survival in HER2+ Breast Cancers

Caitlin Andrews, Elizabeth A Mittendorf, Juhee Song, Kelly K Hunt, Aysegul A Sahin, Yun Wu. UT MD Anderson Cancer Center, Houston, TX.

Background: HER2 + breast cancer $(\mathrm{BC})$ is an aggressive subtype that accounts for $15-20 \%$ of all BC. Patients with HER $2+$ BC benefit from treatment with trastuzumab, a monoclonal antibody targeting the HER 2 protein. Recent studies evaluating trastuzumab combined with chemotherapy (CT) in the neoadjuvant setting have reported complete pathologic response $(\mathrm{pCR}$ ) rates ranging from $40-60 \%$. Achieving $\mathrm{pCR}$ after neoadjuvant therapy (NAT) is a strong indicator of therapy benefit, and therefore, it is prognostic for favorable long-term outcome. To date, no biomarkers have been identified to reliably predict $\mathrm{pCR}$. Recent studies have shown that both tumor characteristics, such as grade and proliferation rate as well as host response, including immune infiltrate, play roles in the response to NAT. This study was undertaken to determine the role of TIL in predicting response to NAT with trastuzumab and anthracycline/taxane in patients with HER2+ BC

Design: A single H\&E stained slide was obtained from the diagnostic core biopsy from 99 HER2+ patients who received NAT with trastuzumab and anthracycline/taxane. TIL were evaluated using the recommended consensus guideline from the International TILs Working Group. Additional staining with anti-cytokeratin and anti-CD45, a panleukocyte antibody, was performed in difficult cases when TIL could not be accurately assessed on the H\&E. Stromal TIL (sTIL) were defined as mononuclear cells within the stroma not in direct contact with tumor cells and were categorized as $<50 \%$, or $\geq 50 \%$ of the tumor stromal area. Statistical analyses were performed to correlate sTIL with pCR and survival.

Results: Patient age at diagnosis ranged from 32-87 yrs (median 52 yrs). 67 (68\%) were white, 32 non-white. A significant sTIL infiltrate, defined as $\geq 50 \%$ sTIL, was identified in $11 \%(11 / 99)$ of the cases. In these cases, $73 \%(8 / 11)$ achieved a pCR. Among cases with $<50 \%$ sTIL infiltrate, $48 \%(42 / 88)$ achieved a pCR $(\mathrm{p}=0.118)$. High sTIL was also associated with better overall survival and recurrence-free survival [Figure 1].
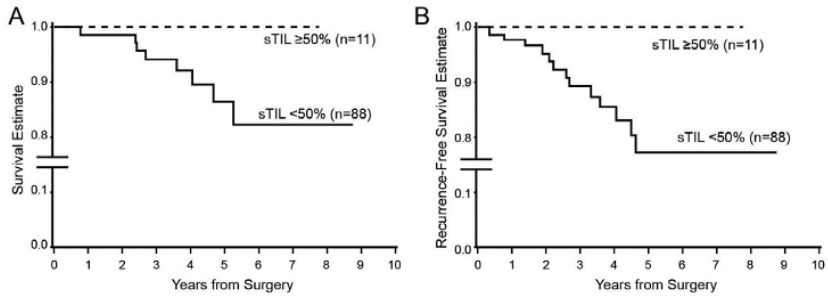

Figure. Kaplan-Meier survival curves for patients with HER2+ breast cancer in relation to STIL in pre-neoadjuvant diagnostic core biopsy.

Conclusions: sTIL may serve as a predictor for pCR in HER2+ BC after trastuzumab and anthracycline/taxane-based therapy. High sTIL is correlated with better overall survival and recurrence-free survival.

107 Programmed Cell Death Ligand 1 (PD-L1) Expression in Estrogen Receptor Positive (ER+) Invasive Breast Cancers (BC): A Pilot Study of 148 Cases

Javier Arias-Stella, Brie Kezlarian, Laura Favazza, Daniel Schultz, Dhananjay A Chitale. Henry Ford Hospital, Detroit, MI

Background: T-cell inhibitory molecule PD-L1 expression by tumor cells protects the tumor from destruction by cytotoxic T cells. Recent early-phase trials in different carcinomas targeting the PD-L1 have shown clinical efficacy that correlated PD-L1 tumor expression and responses. However, data on PD-L1 expression in breast cancer is limited and on $\mathrm{ER}+\mathrm{BC}$, even more scarce. The aim of this study was to determine the frequency of PD-L1 overexpression in $\mathrm{ER}+\mathrm{BC}$ and its correlation with tumor infiltrating lymphocytes (TILs) and clinicopathologic characteristics including molecular subtypes. Design: From 148 cases of ER+BC, tissue microarrays (TMAs) were constructed, each case represented in triplicate. Immunohistochemistry was performed using antibody against PD-L1 protein [SP263 Rabbit monoclonal primary antibody, Ventana, Tuscon, AZ) and leukocyte common antigen (LCA) pan-T lymphocyte marker (DAKO, Carpinteria, CA) to assess TIL density accurately. PD-L1 expression was scored in the cytoplasm or on the membrane of the tumor cells as follows : $0:<1 \%, 1: 1-5 \%, 2$ : $5-10 \%, 3:>10 \%$. The number of positive lymphocytes per core was counted. Multiple clinical and morphologic parameters including histologic type, Nottingham grad,e tumor size, TMN stage, angiolymphatic invasion, and receptor status were recorded from electronic pathology report. Statistical analysis was performed using t- test and ANOVA for correlations ( $p$-value $<0.05$ significant).

Results: Out of 148 cases of breast carcinomas, there were 97/148 (66\%) Luminal A (ER+/PR+/HER-2-/Low MIB1), and 51/148 (34\%) Luminal B (ER+/PR+/HER-2+ or high MIB1 labeling $>15 \%$ ). The average tumor size was $1.7 \mathrm{~cm}$ (SD: 1.0 , range: 0.4-8.5 cm, median: $1.5 \mathrm{~cm})$. There were $48 / 148(32 \%)$ grade $1,83 / 148(56 \%)$ grade 2 , and $17 / 148(12 \%)$ grade 3 tumors. All case were ER+(100\%), 130/148 (88\%) PR+, $8 / 148(5 \%)$ HER $2+$ tumors. PD-L1 expression in tumor cells was noted in only 9/148 $(6 \%)$ cases vs $103 / 148(70 \%)$ in the TIL. There was a statistically significant correlation between PDL-1 lymphocytes in high grade tumors $(p=0.0476)$ and luminal B molecular subtype $(p=0.0062)$

Conclusions: PD-L1 expression in the tumor cells was upregulated in a minority of $\mathrm{ER}+\mathrm{BC}$ in this pilot cohort. However, PD-L1 expression in the TIL was frequently found in high grade ER+BC . PD-L1 upregulation did correlate with strong local immune response in this cohort. Our findings suggest that further characterization of the $\mathrm{ER}+\mathrm{BC}$ immune microenvironment may yield targets for immune-based therapy and prevention in a subset of $\mathrm{BC}$ patients.

108 Outcomes of Benign Intraductal Papillomas (IDP) Diagnosed on Core Biopsy: Do All Need to Be Excised?

Jaya R Asirvatham, Angela Wu, Julie Jorns. University of Michigan, Ann Arbor, MI. Background: The necessity of excising IDPs diagnosed on core biopsy is controversial due to varying upgrade rates on excision (0-29\%). We studied the clinicoradiopathologic features associated with IDP upgrade at our institution. 
Design: An electronic database search (2007-2015) for all core biopsies of IDPs was performed. Biopsy and excision slides were reviewed, and clinicopathologic features between papillomas with upgrade (UP) and no upgrade (NUP) on excision to atypia/ carcinoma, both within the IDP and adjacent breast tissue, were compared.

Results: 83 IDPs from 79 women (mean age 52) were included. Residual IDP was present in $86 \%(71 / 83)$ of excisions. $11 \%(9 / 83)$ were UPs, and the majority had atypia/ carcinoma in the adjacent tissue (not within the IDP). $67 \%$ (6/9) of UPs had a history of or concurrent carcinoma (either contralateral or in a separate quadrant) versus $14 \%(10 / 74)$ of NUPs. The three with upgrade within the IDP had a mean size of $0.7 \mathrm{~cm}$ and 1.5 $\mathrm{cm}$ on biopsy and excision, respectively, versus $0.4 \mathrm{~cm} / 0.7 \mathrm{~cm}$ for all others $(\mathrm{n}=80)$; however, the overall mean size of UPs and NUPs were identical $(0.4 / 0.7 \mathrm{~cm}$ on core/ excision). There was no significant difference in age, menopausal status, radiologic mass, calcifications, detection mode, sampling method, percentage of cores involved, radiopathologic concordance and multiplicity between the groups.

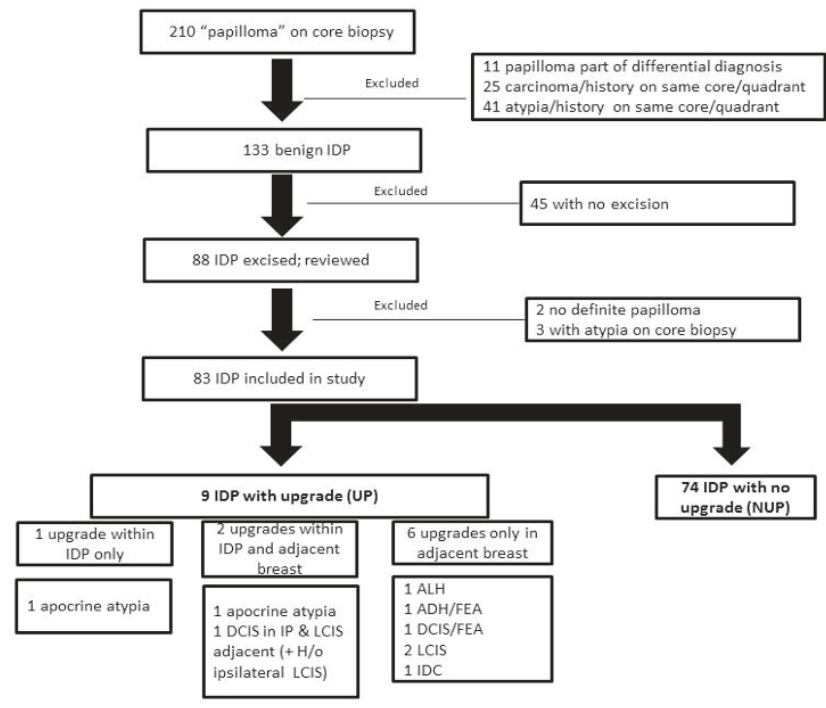

Conclusions: The vast majority of IDPs are not upgraded on excision, and the majority (67\%) of UPs had a history of/concurrent contra/ipsilateral carcinoma. These findings support that excision of IDPs should be decided on a case-by-case basis.

109 Integration of Androgen Receptor (AR) Expression to Predict Survival for Triple-Negative Breast Carcinoma (TNBC)

Kristine Astvatsaturyan, Yong Yue, Shikha Bose. Cedars Sinai Medical Center, Los Angeles, CA.

Background: TNBC is a heterogeneous group of aggressive cancers that represent a treatment challenge. Gene expression analyses have shown that AR is overexpressed in a subset and that these patients may benefit from anti-androgen therapy. This study aims to determine the prognostic value of AR in predicting survival of TNBC patients. Design: 114 consecutive invasive TNBCs diagnosed from 2008-2012 were retrieved. Patients ranged in age from 28-92 yrs (median=57). All carcinomas were surgically excised as primary treatment. Follow up was obtained from the tumor registry. Paraffing embedded formalin fixed sections were immunostained with EFGR, CK5/6 and AR antibodies and evaluated by two pathologists, concordant results were recorded. Cutoff values of immunoexpression were determined by time-dependent ROC curves associating survival outcome. Results were correlated with pathologic variables using a uni and multivariate Cox analysis. Patient risks were identified by Kaplan-Meier curves and log-rank tests.

Results: AR was positive in $52 \%$ cases, $25 \%$ showing strong positivity in $>25 \%$ tumor cells. EGFR $(\mathrm{p}=0.026)$ and $\mathrm{AR}(\mathrm{p}=0.073)$ correlated significantly with disease-free survival (DFS) on multivariate Cox analysis. Patients were categorized into low (1), intermediate (2) and high (3) risk groups. Group 1 with high AR $(>25 \%)$ and low EGFR ( $<15 \%)$ expression showed the best outcome. Group 2 included TNBCs with low EGFR/AR expression, and those with high EGFR/AR expression. A statistically significant worse outcome (7.9 months) was observed for Group 3, low AR and high EGFR expression $(\mathrm{p}=0.014)$.

\begin{tabular}{|l|l|l|l|l|l|}
\hline Risk Groups & Patient No. (\%) & DFS Months & Events\% & EGFR $\%$ & AR $\%$ \\
\hline Low & $5(4.4 \%)$ & 16 & 0 & 3.6 & 81.7 \\
\hline Intermediate & $55(48.2 \%)$ & 11.8 & 12.7 & 30.5 & 27.1 \\
\hline High & $54(47.4 \%)$ & 7.9 & 31.5 & 58.5 & 3.0 \\
\hline
\end{tabular}

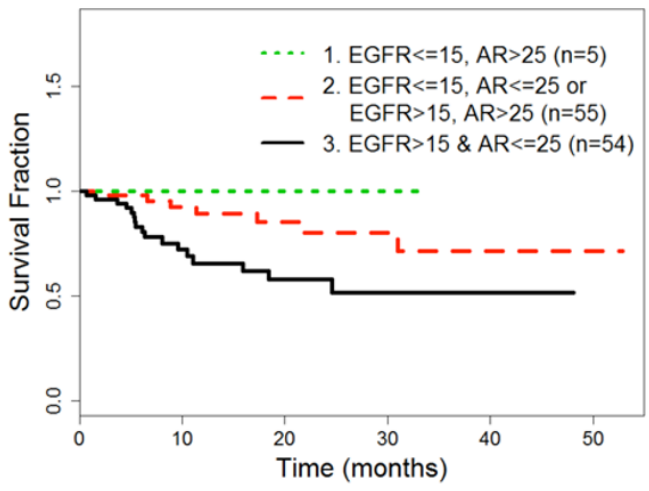

Conclusions: $25 \%$ of TNBCs showed strong AR immunoexpression. AR, in association with EGFR immunoexpression is useful in stratifying TNBCs into different risk groups. Low AR with high EGFR significantly reduces survival probability suggesting aggressive disease. AR immunoexpression may also be used to identify patients for antiandrogen targeted therapies.

110 Histologic Features of Lymph Node Metastases and Their Prognostic Significance in Node Positive Breast Cancer

Sura M Aziz, Elisabeth Wik, Goril Knutsvik, Tor Audun Klingen, Ying Chen, Benedicte Davidson, Hans Aas, Turid Aas, Ingunn Stefansson, Lars A Akslen. University of Bergen, Bergen, Norway; Haukeland University Hospital, Bergen, Norway; Vestfold Hospital Trust, Tønsberg, Norway; Akerhus University Hospital, Lørenskog, Norway. Background: Lymph node (LN) metastasis is a powerful prognostic factor in breast cancer. However, the prognostic impact of certain nodal tumor features, like number of positive nodes, metastatic tumor diameter (metTD), tumor location, nodal afferent and efferent vascular invasion (AVI, EVI), and extranodal extention (ENE) are not fully understood. Here, we examined node-related tumor features and their prognostic value. Design: A population- based cohort of node positive breast cancer $(n=218)$, as part of the prospective Norwegian Breast Cancer Screening Program (1996-2009), was used. Sections from the positive nodes were recorded for the largest metastatic TD, intranodal tumor location, nodal AVI and nodal EVI. Extranodal extension and its extent, by the number of ENE focuses and the largest horizontal (HD) and vertical diameter (VD) of the largest ENE focus, were recorded. Survival analysis (log-rank test) examined the impact of these features on disease free survival (DFS; 72/218) and breast cancer specific survival (BCSS; 43/218).

Results: High number of positive LN ( $\geq 4$ positive nodes), presence of nodal EVI, high number of ENE focuses $(\geq 4)$, and $\mathrm{HD} \geq 3 \mathrm{~mm}$, were associated with large primary tumor diameter $(P 0.001,0.05,0.04,0.03$, respectively). Metastatic TD was associated with histologic grade 3, ER negativity, and HER2 positivity $(P 0.006,0.039,0.001$, respectively). Univariate survival analysis (log-rank test) showed that number of positive LN, AVI, EVI, number of ENE focuses and its HD were significantly prognostic in DFS and BCSS. Multivariate survival analysis (Cox's proportional hazards method) showed an independent prognostic value of HD in predicting DFS and BCSS in addition to number of positive $\mathrm{LN}$ and histologic grade of primary tumor $(P 0.01,0.005,0.002$, respectively for DFS; $P 0.02,0.008,0.002$, respectively for BCSS).

Conclusions: Several nodal tumor features were significant prognostic factors. Of particular interest, the extent of ENE by its largest horizontal diameter was independently prognostic and should be considered in line with nodal tumor burden in treatment decisions.

111 Lipophyllodes of the Breast: A Multi-Institutional Study of 22 Cases with Clinicopathological Correlations, Molecular Insight and Follow Up Carlos E Bacchi, Sheila Wludarski, Janez Lamovec, David Ben Dor, Giovanna De Maglio, Stefano Pizzolitto, Elisa Ober, Fabrizio Zanconati, Giovanni Falconieri. Consultoria em Patologia, Botucatu, Sao Paulo, Brazil; Institute of Oncology, Ljubljana, Slovenia; Barzilai Medical Center, Askelon, Israel; University Hospital, Udine, Italy; University Hospital, Trieste, Italy.

Background: There are few studies addressing mammary lipophyllodes tumors (LPT), e.g., phyllodes tumors featuring adipose differentiation. Series with available special stains and follow-up are also limited. We have studied 22 cases of LPT, analyzing their clinicopathologic features along with available follow-up.

Design: Tissue material of 22 LPT was retrieved from authors' departmental files. To be included in the series cases, the adipose component needed to be atypical and preponderant; mature fat changes in ordinary phyllodes tumors were not considered. All cases were tested for cytokeratins, S100 protein, CD34, Bcl-2, MDM2 and desmin. Results: Patients were all females, 21-69 years of age, average 45 years; LPT size range was $1.6-30 \mathrm{~cm}$, average $9.7 \mathrm{~cm}$. Microscopically, LPT segregated as follows: atypical lipomatous tumors/ALT, 8 cases; myxoid, 6; pleomorphic/poorly differentiated/ round cell, 7; and dedifferentiated liposarcoma (LS), 1. Immunohistochemistry was positive for $\mathrm{S} 100$ and CD34 in ALT; desmin in 2 pleomorphic LS cases with evidence of rhabdomyoblastic differentiation. MDM2 positivity was focally seen in 1 LS case. Follow-up was available in 8 cases. 


\begin{tabular}{|l|l|l|l|}
\hline Age & $\begin{array}{l}\text { Salient gross features } \\
\text { and size, } \mathrm{cm}\end{array}$ & $\begin{array}{l}\text { LPT microscopic } \\
\text { features }\end{array}$ & $\begin{array}{l}\text { Treatment and follow up } \\
\text { (y, years; NED, no evidence of disease) }\end{array}$ \\
\hline 60 & Necrotic; 30 & Pleomorphic LS & $\begin{array}{l}\text { Pulmonary metastases on admission; dead } \\
\text { of tumor }\end{array}$ \\
\hline 47 & Nodular, 4 & Pleomorphic LS & $\begin{array}{l}\text { Mastectomy; metastases at neck and } \\
\text { lung, after 2 and 3y, respectively; } \\
\text { metastasectomy; NED 6 y after initial } \\
\text { diagnosis }\end{array}$ \\
\hline 39 & Nodular, 4 & Myxoid LS & $\begin{array}{l}\text { Excision; recurrent tumor after 4 y, treated } \\
\text { with mastectomy; NED 12 y after initial } \\
\text { diagnosis }\end{array}$ \\
\hline 45 & Nodular, 4.2 & Myxoid LS & $\begin{array}{l}\text { Excision; recurrent tumor after 23 y; } \\
\text { mastectomy; NED, 9 y }\end{array}$ \\
\hline 52 & Nodular, 4.2 & Myxoid LS & Excision; NED, 8 y \\
\hline 63 & Lobulated, 2.5 & ALT & Excision; NED, 15 y \\
\hline 46 & Nodular, 2.5 & ALT & Excision; NED, 2 y \\
\hline 30 & Lobulated, 3 & Myxoid LS & Excision; NED, 5y \\
\hline
\end{tabular}

Conclusions: SCs have a distinct gene expression profile when compared to IDC-NOS. Some SCs express ER at low levels, detectable by both IHC and qPCR. Results of prognostic/predictive assays must be interpreted in the entire clinical and pathologic context. Per SOP, RS results are not reported for SCs.

\section{HER2 FISH Equivocal Category: Does Retesting Resolve This} Clinical Grey Zone?

Morgan Ballard, Sucheta Srivastava, Robert B West, Kristin C Jensen, Richard K Sibley, Kimberly Allison. Stanford Healthcare, Stanford, CA; VA Healthcare System, Palo Alto, CA.

Background: Recent reports show an increase in cases with HER2 FISH equivocal results, a category for which the 2013 HER 2 testing guidelines recommend retesting. This study examines whether retesting provides resolution of equivocal results and how this category is clinically managed.

Design: Our database of HER2 FISH results was searched for cases considered equivocal by 2013 guideline criteria from 2009-2015. Available clinico-pathologic data including patient age, grade, ER status, HER2 IHC status and HER2 retesting results were collected. Treatment information was also collected.

Results: From 2009-September 2015, 79 cases were classified as equivocal based on the new guidelines, $52 \%$ of which were originally classified as negative pre-2013 guidelines adoption. FISH equivocal cases were mainly ER+ $(77 \%)$ and grade $2-3$ (83\%). Of cases with concurrent HER2 IHC $(63 \%), 10 \%$ were IHC positive, $34 \%$ negative, $56 \%$ equivocal. Of FISH equivocal results, $38 \%$ were on initial core biopsies and $62 \%$ on surgical specimens ( $24 \%$ were post-neoadjuvant therapy). $40 \%$ of cases reported as FISH equivocal were retested. Of FISH equivocal core biopsies retested on the surgical specimen or lymph node metastasis, $46 \%$ had the same result, $23 \%$ were positive, and $31 \%$ negative. For resection specimens with FISH equivocal results, retesting on additional blocks from the same specimen had $56 \%$ same result, $11 \%$ positive, $33 \%$ negative. Overall conversion to a positive result occurred in $17 \%$ of the retested cases. The majority of these cases $(75 \%)$ had retest ratios minimally elevated above the 2.0 cutoff, due to slight increases in the mean HER2 signals/cell and slight decreases in the mean CEP17 signals/cell (Table 1). The cases with a positive retest result were often strongly ER+, grade 2-3 with high Ki67. Of cases called equivocal $29 \%$ received HER 2 targeted therapy.

Conclusions: HER2 FISH equivocal cases are frequently retested with the same results. However conversion to a positive FISH result after an initial equivocal result on a core biopsy can occur in close to a quarter of cases. This is typically due to subtle shifts in the mean HER2 and CEP17 counts, with ratios just above the positive threshold.

Table 1: Positive retests of other genes that have been suggested to be good alternatives to the CEP17 probe Results: The analysis of copy number alterations (CNA) showed $0-6 \%$ alterations in $17 \mathrm{p}$ genes (WRAP53 2\%; FOXN1 4\%; ACACA 6\%; CYRBA1 6\%; CTNS 0\% respectively). Similarly CNA frequencies were in 17 q genes ranged from $5-8 \%$ (TOP2A $6 \%$; RAD $51 \mathrm{C} /$ FANC0 8\%; PYCR1 5\%; ACOX1 7\%; USH1G 7\% respectively). Alterations in SMS, RARA and TP53 were noted in $2 \%, 7 \%, 32 \%$ respectively. Of note, most of the alterations were amplification events except for TP53. TP53 harbored missense and truncating mutations and deep deletion, amplification was noted in only 1 of 951 cases. Conclusions: There is a remarkable instability in the genomic content of chromosome 17 centromeric region. The use of CEP17 probe for normalization of chromosome number in HER2 scoring can introduce significant biases in the analysis. Alternative probes such as SMS and RARA are also frequently amplified. TP53 amplification was rarely observed however mutations were very common. The study validates the use of single HER2 probe analysis for detection of copy number, as recommended in the new ASCO-CAP guidelines.

113 Secretory Carcinoma of the Breast: Molecular Characterization of 12 Cases by Standardized Quantitative RT-PCR Analysis

Helen Bailey, Debbie McCullough, Frederick L Baehner, Carolyn Mies. Genomic Health, Inc., Redwood City, CA.

Background: Secretory carcinoma (SC) is a rare subtype of invasive ductal carcinoma with fewer than 200 cases reported to date. SC was originally called "juvenile" carcinoma, as many cases occur in females and in males $<30$ years old. Most SCs are ER, PR, and HER2-negative ("triple negative"), but some express low levels of ER as detected by immunohistochemistry (IHC). Despite this biomarker profile, $\mathrm{SC}$ is known to have an indolent clinical course; most patients are cured with surgery, and the few patients who develop metastasis rarely die of their cancer. To help shed light on this paradoxical clinical behavior, we compared quantitative gene expression data for IHCER-positive SCs ( $\mathrm{N}=12)$ to ER-positive invasive ductal carcinoma, NOS (IDC-NOS; $\mathrm{N}=427,844$ ), sent to us for 21 -gene Recurrence Score $\mathbb{R}$ (RS) testing.

Design: We evaluated 12 invasive carcinomas with classic morphologic features of the SC subtype that were IHC-ER-positive at the submitting site (2009-2015). Gene expression is quantitated on a scale from 2 to 15 (relative to reference genes), where each one-unit increment is associated with an approximate two-fold change in expression. Results: SC patients (11 female, 1 male) ranged from 26 to 79 yrs (mean 52 yrs), similar to the age range of IDC-NOS (mean 58 yrs). There were distinct differences in gene expression. Mean ER expression was 6.5 (range 5.3 to 8.2), lower than that seen in IDC-NOS (mean 10.0, range 2.4 to 15.5). Mean PR expression was 4.1 (range 2.9 to 6.4), lower than that seen in IDC-NOS (mean 7.3, range 2.0 to 13.7). Compared to IDC-NOS, mean expression of the proliferation genes (Ki-67, STK15, SURV, CCNB1, MYBL2) was 1 unit lower, mean expression of the ER gene group (BCL2, CEGP1, ER and PR) was 2.8 units lower, and mean expression of the invasion genes (STMY3 and CTSL2) was 1 unit higher in SC. Mean HER2 expression was lower in secretory carcinoma (8.7) than in IDC-NOS (9.2). No differences were seen in other assay genes (CD68, GSTM1, BAG1, and GRB7).

\begin{tabular}{|l|l|l|l|l|l|}
\hline $\begin{array}{l}\text { Initial } \\
\text { Ratio }\end{array}$ & $\begin{array}{c}\text { Retest } \\
\text { Ratio }\end{array}$ & $\begin{array}{l}\text { Initial Mean } \\
\text { HER2 signals/cell }\end{array}$ & $\begin{array}{l}\text { Retest Mean } \\
\text { HER2 signals/cell }\end{array}$ & $\begin{array}{l}\text { Initial Mean } \\
\text { CEP17 signals/cell }\end{array}$ & $\begin{array}{l}\text { Retest Mean } \\
\text { CEP17 } \\
\text { signals/cell }\end{array}$ \\
\hline 1.29 & 2.09 & 4.32 & 5.52 & 3.36 & 2.64 \\
\hline 1.44 & 2.07 & 4.16 & 5.29 & 2.88 & 2.54 \\
\hline 1.46 & 2.2 & 5.10 & 7.28 & 3.5 & 3.28 \\
\hline 1.18 & 5.56 & 4.16 & 10 & 3.52 & 1.80 \\
\hline
\end{tabular}

115 Axillary Lymph Node FNA vs CNB in Breast Cancer Patients: A Blinded Prospective Study

Gonzalo Barraza, Robert L Schmidt, Elke Jarboe, Jason M Wells, Nicole Winkler, Rachel E Factor. University of Utah Health Sciences Center, Salt Lake City, UT.

Background: The status of lymph nodes is the most important prognostic factor for patients with invasive breast cancer. NCCN guidelines recommend sampling suspicious axillary lymph nodes either by core needle biopsy (CNB) or fine needle aspiration (FNA). At our institution, radiologists prefer CNB over FNA due to workflow difficulties, including coordination with cytopathologists to perform rapid on site evaluation (ROSE). Numerous retrospective studies, but only one prospective study, have looked at the diagnostic accuracy of FNA vs CNB. Our study prospectively investigates the diagnostic accuracy of FNA and CNB performed concurrently on enlarged axillary lymph nodes in breast cancer patients using ThinPrep without ROSE. Design: This is a prospective, blinded study, which is continuing to enroll. At this time, 52 patients could be evaluated for diagnostic accuracy and 63 patients could be evaluated for interobserver agreement. Each patient had an enlarged lymph node concurrently sampled by ultrasound-guided FNA and CNB. FNA samples were deidentified, placed in CytoLyt $\mathbb{R}$ and one ThinPrep was prepared. FNA samples were reviewed independently by three blinded cytopathologists, reporting both adequacy and diagnosis. All patients underwent lymph node dissection during resection. Interrater agreement was assessed using actual agreement and chance-corrected agreement (Cohen's kappa). Diagnostic accuracy of FNA was compared to cases with tissue results. Average accuracy was obtained by meta-analysis. Heterogeneity was assessed using Higgins $I^{2}$ statistic.

Results: For ease of interpretation, adequacy was unsatisfactory or satisfactory. Atypical was considered negative, and suspicious was considered malignant. The FNA adequacy rate was $98.4 \%$ (95\% CI: 91-100). The overall interrater agreement was $90 \%$. Chance corrected agreement (kappa) was 0.78 (95\% CI: 0.66-0.90). Diagnostic accuracy of CNB was $100 \%$. The diagnostic accuracy of FNA was lower than the diagnostic accuracy of CNB. For FNA, the average sensitivity was $82 \%$ (95\% CI: 73-89) and the average specificity was $96 \%(95 \% \mathrm{CI}: 87-100)$. The possibility that false negative FNAs were associated with micrometastases on the cores was explored, however, all cases were macrometastases. 
Conclusions: FNA using ThinPrep alone has acceptable diagnostic accuracy compared to $\mathrm{CNB}$, but it suffers from a higher false negative rate. This could be improved with the use of cell blocks for atypical cases, something we plan to investigate in the future.

116 Computational Image Analysis of Breast Biopsy Specimens with Atypia - Through the Looking Glass of Diagnostic Concordance among Pathologists

Francisco Beca, Irshad Humayun, Donald L Weaver, Joann Elmore, Andrew H Beck. Beth Israel Deaconess Medical Center, Boston, MA; Harvard Medical School, Boston, MA; University of Vermont School of Medicine, Burlington, VT; University of Washington School of Medicine, Seattle, WA.

Background: Approximately 1.2 million women undergo a breast biopsy each year in the United States and are diagnosed with a non-invasive breast lesion. Non-invasive breast lesions are challenging, especially in cases of atypia and DCIS, as the Breast Pathology (B-Path) study recently confirmed (Elmore J et al., 2015). However, the causes of discordance in the assessment of atypia are largely unknown. Therefore the goal of this study is to use computational image analysis to identify histologic features in breast biopsies with atypia that predict the agreement between pathologists.

Design: We performed a quantitative computational analysis using 30 digital tissue slide images of the 15 most concordant and the 15 most discordant cases with atypia from the B-Path study. After identifying regions of interest (ROIs), we computationally extracted and quantified 1300 image-level features (including morphometric features of nuclei and nuclear spatial distribution and measures of epithelial and stromal morphology). Afterwards, we built a multivariate image feature-based discriminatory model to classify each selected ROI as belonging to a high versus low agreement case. We performed model validation by the leave-one-patient-out cross validation method and assessed the model's performance by computing the area under the ROC.

Results: Sixteen nuclear and region level features showed significantly different patterns between high vs low concordance (FDR $<5 \%$ ) and were included in our model. The area under the ROC for classification performance was 0.72 in cross-validation. Top performing features included reduced variability in epithelial nuclear length, increased variability in epithelial nuclear crowdedness, and increased relative border of stromal region to epithelial region, all of which were indicative of greater concordance.

Conclusions: Through our computational analysis we identified morphologic features associated with diagnostic concordance among pathologists in the evaluation of breast biopsies with atypia. Additionally we built a model which in the future may assist in the identification of cases for referral for a second-opinion/external review.

117 Evaluation of EZH2 Protein Expression in Normal Epithelium as a Marker of Risk of Breast Cancer Development - Results from the Nurses' Health Study

Francisco Beca, Kevin Kensler, Benjamin Glass, Rulla M Tamimi, Andrew H Beck. Beth Israel Deaconess Medical Center, Boston, MA; Harvard Medical School, Boston, MA; Harvard T.H. Chan School of Public Health, Boston; Brigham and Women's Hospital, Boston, MA.

Background: EZH2 is a component of a Polycomb Group that is involved in stem cell renewal and carcinogenesis. Increased EZH2 expression is associated with aggressiveness in breast cancer and has been previously been suggested to identify normal breast epithelium at increased risk for breast cancer development (Kunju LP, et $\mathrm{al}, 2011)$. This association has not previously been evaluated on a large epidemiologic cohort. The objective of this study was to evaluate the association of EZH2 protein expression in normal breast epithelium and stroma with the development of subsequent breast cancer.

Design: We examined the association between EZH2 protein expression and subsequent breast cancer risk in a nested case-control study of benign breast disease (BBD) and breast cancer within the Nurses' Health Study. We performed immunohistochemistry on tissue microarrays and evaluated EZH2 expression quantitatively in the normal breast epithelium and stroma using Definiens Tissue Studio image analysis program. Logistic regression was used to compute the odds ratio (OR) for the associations described. All reported results have been adjusted for matching factors and concomitant BBD diagnosis.

Results: Three hundred and forty three women were included in our final dataset (74 cases and 269 controls). Women with a normal breast biopsy in which more than $20 \%$ epithelial cells expressed EZH2 had a significantly increased risk of developing breast cancer (OR: 2.67, 95\% CI: 1.24-5.76) compared to women with absent/low EZH2 epithelial expression, and the risk of developing breast cancer increased (OR: 1.20, 95\%CI: 1.04-1.38, p-value 0.010) with each 5\% increase in EZH2 expression.

Conclusions: These results provide further evidence that EZH2 expression in the normal breast epithelium is associated with breast cancer risk and might be used to assist in risk stratification of women with benign breast biopsies.

118 Concordance in the Cloud- A Multidisciplinary Software Tool for Breast Core Biopsy Correlation

Michael W Bennett, Tim Manning, Brian Kelly, Noel Murphy, Paul Walsh, Tara Jane Browne, Fionnuala O'Connell. Cork University Hospital, Cork, Ireland; nSILICO, Cork, Ireland; Health Innovation Hub, Cork, Ireland.

Background: Clinical-Radiologic-Pathologic correlation is the cornerstore of patient management following breast core biopsy and a forum to evaluate this is an essential part of a Breast Service. A 'triple assessment' breast multidisciplinary team meeting (MDTM) provides this forum where concordance/discordance is established and an appropriate outcome generated. This MDTM is associated with high volume paperwork. Significant data is generated but meaningful data-mining including audit of outcomes, trends and performance indicators is complex. Due to high case throughput including multiple and/or bilateral lesions potential for error exists. To address these limitations we developed a cloud-based software application that assists in coordination and execution of a breast core MDTM.

Design: Our center is a large academic center with approximately 600 breast cancer diagnoses per year. The breast core pathway was process-mapped from sampling through reporting and MDTM. The platform was developed to accommodate all aspects of the process in a user-friendly, cloud-based tool. The tool incorporates multiple fields including case identifiers, clinical, radiology and pathology scores (5-tier scoring system), concordance/discordance, diagnosis, outcome, comment and images. Symbols and 'traffic-light' color codes for recording concordance/discordance, benign, malignant and atypical categories are used and projected at MDTM as a visual management tool. Cases of educational merit are tagged. Deferred cases automatically populate to the next MDTM. Each lesion is uniquely identified to assure safe follow-up. A meeting summary is automatically generated. Retrieval of audit data across multiple paremeters can be automatically generated in graphical format. Past MDTM history is integrated. The system is secure to HIPAA standards and includes a fully traceable audit trial. It allows flexibility in MDT preparation to registered users. This platform was used in in parallel with our preexisting correlation system for validation.

Results: A cloud-based breast MDT tool has been developed that facilitates ease of access, data entry and visual management of concordant/discordant cases with comprehensive data analysis. This tool is as efficient as a traditional breast core correlation model and offers increased safety and quality improvement with potential for cross-institutional patient tracking/ collaboration.

Conclusions: This cloud-based MDT software tool optimises clinical-radiologicpathologic correlation of breast core biopsies in a MDTM context.

\section{Microglandular Adenosis and Risk of Breast Cancer: A Mayo Benign Breast Disease Cohort Study}

Melanie C Bois, Zahraa Al-Hilli, Daniel W Visscher, Tanya L Hoskin, Marlene H Frost, Derek C Radisky, Ruth Kraft, Amy Degnim, Jodi M Carter. Mayo Clinic, Rochester, MN. Background: Microglandular adenosis (MGA) is a very rare form of mammary adenosis characterized by an infiltrative population of glands, lined by a single epithelial layer and lacking a myoepithelial layer. Due to its rarity, the biologic behavior of MGA remains unclear. While MGA is generally considered a benign lesion, it can co-exist with invasive or in situ carcinoma, and molecular genetic data suggest it may be a non-obligate precursor lesion. We evaluated the frequency of MGA and its associated clinical outcomes in a large, well-defined cohort of women with biopsy-proven benign breast disease.

Design: By retrospective pathologic review, breast biopsies containing MGA were identified within the Mayo Clinic Benign Breast Disease Cohort $(\mathrm{N}=13,538$ women with benign breast biopsies at Mayo Clinic, MN between 1967 and 2001). The biopsies were sub-classified into benign breast disease categories including proliferative disease without atypia (PDWA) or with atypia. Follow-up for breast cancer events was obtained from electronic medical records, Mayo Tumor Registry and a study-specific questionnaire. Breast cancer risk was assessed using standardized incidence ratios (SIRs) with the expected number of events calculated using population incidence data from the Iowa Surveillance, Epidemiology, and End Results registry.

Results: MGA was identified in 21 of 13,538 biopsies $(0.2 \%)$ from patients with a median age of 61y (range 45-71y). None of the MGA-positive biopsies had atypical features or concurrent in situ or invasive carcinoma. 19 of 21 MGA-positive biopsies were categorized as PDWA. Among these MGA/PDWA biopsies, with a median followup time of $17 y$ (range 1y to 29y), no patient developed in situ or invasive carcinoma (SIR of 0, 95\% CI: 0-2.87) with 1.29 expected events in the general population based on age, biopsy date and length of follow-up. Two MGA-positive biopsies had concurrent atypical ductal hyperplasia; one of these patients developed an ipsilateral invasive ductal carcinoma two years later.

Conclusions: MGA is a very rare proliferative lesion in women with benign breast disease. In the context of proliferative disease without atypia, MGA appears not to increase breast cancer risk. Additional studies on larger series may clarify the biologic behavior of MGA and determine whether it is a non-obligate precursor of breast carcinoma.

120 Do Breast Papillomas without Atypia on Core Biopsy Require Excision? A 10-Year Single Institution Study and Review of the Literature Christine Bookhout, Sheryl Jordan, Thomas Lawton. UNC Health Care, Chapel Hill, NC. Background: The core biopsy literature considers breast papillomas a risk lesion based on studies showing variable chances of finding associated malignancy on excisional biopsy. Prior studies differ in design and structure, and recommendations for or against surgical excision are inconsistent. Determining the true risk potential of benign papillomas is critical for informing recommendations for management of these lesions. Design: We identified 55 published studies from 1999-2015 analyzing the rate at which breast papillomas on core needle biopsy $(\mathrm{CNB})$ are upgraded after surgical excision and/or clinical and radiologic follow-up, and assessed them for the following confounding factors: 1) no radiologic-pathologic concordance of $\mathrm{CNB}$ results and/or including discordant cases; 2) including CNBs with papilloma plus another risk lesion (e.g. ADH); 3) including risk lesions as upgrades; 4) no comment on the distance of the upgraded lesion from the biopsy site, raising the possibility that the upgraded lesion was unrelated to the papilloma; and 5) no histologic review of biopsies by a breast pathologist. At our institution, with IRB approval, we reviewed records of all breast CNBs performed from 7/1/2004-6/30/2014 with diagnosis of non-atypical solitary papilloma, addressing the above factors and including cases that were either excised or had $>2$ years of follow-up.

Results: At our institution, 60 benign, radiologically concordant papillomas were identified over the 10 -year period. Thirteen subsequently underwent surgical excision 
for symptomatic relief or growth on follow-up imaging; 1/13 (7.7\%) was upgraded to invasive ductal carcinoma (IDC) adjacent to papillary carcinoma. Of 47 cases with $>2$ years of follow-up, $1 / 47$ patients $(2.1 \%)$ was diagnosed with IDC involving the prior biopsy site after 2 years, for an overall upgrade rate of $2 / 60(3.3 \%)$.

In our literature review, 25 studies had no upgrades. Of the remaining 30 studies, 26 had at least one confounding factor. The studies included a total of 3617 papillomas; after attempting to apply the above criteria to each study, $151(4.2 \%)$ were upgraded to malignancy on excision. In the majority of these studies, however, all criteria could not be retrospectively applied, raising the possibility that the true upgrade rate is even lower. Conclusions: Our institutional data and literature review suggest that benign papillomas, when concordant and adequately sampled, have a relatively low risk of malignancy. Close radiologic follow-up for benign papillomas with radiology-pathology concordance can prevent unnecessary surgical excisions.

\section{Interobserver Agreement of Breast Cancer IHC4 after "Score the} Core" Training

Alexander Borowsky, Ronald Balassanian, Christina Yau, Jesse A Engelberg, Carlie K Thompson, Hanna EG Retallack, John W Bishop, Robert Cardiff, Philip M Carpenter, Yunn Yi Chen, Brian Datnow, Farnaz Hasteh, Gregor Krings, Fritz Lin, Yanhong Zhang, Linda S Lindstrom. UC Davis, Sacramento, CA; UCSF, San Francisco, CA; UCSD, La Jolla, CA; USC, LA, CA; UCI, Irvine, CA; Karolinska, Stockholm, Sweden. Background: IHC4 score combines ER, PR, HER2, and Ki-67 status to provide a semiquantitative prognostic score for breast cancer. Few previous efforts have been made to measure accuracy or decrease interobserver variance. We measured the interobserver variance across four sites and nine pathologists after web-based training.

Design: Study pathologists first completed "Score the Core" training (HumPath; Jul 29, 2015). A retrospective set of 727 invasive breast carcinomas were sectioned and stained in one laboratory for H\&E, ER, PR, Her2, and Ki67. Case assignments were randomized with every case read by two pathologists. Each case was provided as a complete set (H\&E, ER, PR, Her2, and Ki67) and scored: ER and PR by percent at each intensity level (H-score components); Her2 using standard guidelines; and Ki67 by percent positive. Additional comments and no-score were permitted if the quality of a slide was insufficient.

Results: Interobserver variability using a Kappa statistic method showed: ER: 0.94; PR: 0.84; Her2: 0.91 . For $\mathrm{H}$-score comparisons, a histogram comparing read A to read B for every case (Figure 1a) shows the distribution of $\mathrm{H}$ scores as well as a comparison of the scoring. Deviation (delta) between A and B were also mapped (Figure 1b) showing a roughly normal distribution with very few cases in the tails. Discrepancies were due to data entry errors, technical issues such as poor tissue adherence, or very light staining. One of the 9 pathologists was the most discrepant, and review of the training $\log$ on Score the Core showed that this individual had the longest time in training and poorest test results.

Conclusions: Overall, interobserver variability was extremely low in this group of expert pathologists after web-based training. Kappa statistics of greater than 0.81 are typically regarded as "almost perfect" agreement. Figure 1 illustrates the full range of $\mathrm{H}$ score variation. Experts at multiple sites trained with the Score the Core tool can provide high precision IHC quantitation suitable for clinical decision making.
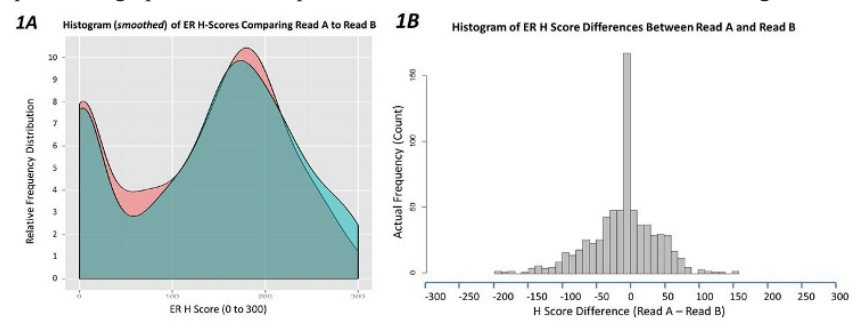

122 Phenotype and Immunophenotype Analysis of Gene Expression Defined "Indolent Risk" Breast Cancers

Alexander Borowsky, Carlie K Thompson, Ronald Balassanian, Christina Yau, Yunn-Yi Chen, Gregor Krings, Bo Nordenskjold, Olle Stal, Tommy Fornander, Laura $J$ Esserman, Laura J van't Veer, Linda S Lindstrom. UC Davis, Sacramento, CA; UCSF, San Francisco, CA; Karolinska, Stockholm, Sweden; Linköping University, Linköping, Sweden.

Background: Prospective identification of indolent or very low risk breast cancers may reduce overtreatment. Using a previously developed gene expression score/signature defining an indolent risk (IR) threshold (manuscript in preparation), we evaluated archived FFPE tumor samples from the 1976-90 Stockholm Tamoxifen (STO) trial of post-menopausal breast cancer patients who were randomized to tamoxifen (Tam) versus not, and followed for more than 20 years. Here we assessed the phenotype and immunophenotype of these IR cases.

Design: 727 FFPE tumor samples from the STO trial of patients with node negative breast cancer and tumor size less than $30 \mathrm{~mm}$, were sectioned and stained with $\mathrm{H} \& \mathrm{E}$, ER, PR, Her2 and Ki67 in a single laboratory. Gene expression analysis was available in 652 , and $15 \%(\mathrm{~N}=98)$ met the threshold for "indolent risk" (Figure 1). Breast cancer-specific survival was assessed by Kaplan-Meier analyses and multivariate Cox proportional hazard modeling. Phenotype, including grade and cell type, receptor (ER, PR, HER2) and Ki-67 status as well as semi-quantitative IHC scoring (H-score) was recorded by a team of expert pathologists.

Results: This subset had significantly lower risk of disease-specific death in multivariate Cox analysis. Most but not all cases in this subset were low grade, ER positive, and had higher $\mathrm{H}$ scores. This group was enriched for tubular carcinoma, lobular carcinoma, and invasive cribriform carcinoma, but mucinous carcinoma was excluded by this IR definition. No special type invasive ductal carcinomas were also characterized by common features. Quantitative IHC analysis, including a calculated IHC4 were also evaluated and correlated.

Conclusions: A gene expression definition of IR identified a subset of breast cancers in a unique retrospective cohort from the STO trial. The tumor phenotypes and immunophenotypes were evaluated. We found significant correlations between special types as well as features of these special types in the subset, suggesting that morphologic phenotypes can be used in conjunction with $\mathrm{IHC} 4$ and gene expression to prospectively identify IR breast cancers.

\section{Kaplan Meier Plot: Breast Cancer Specific Survival Indolent Risk Compared to MammaPrint High/Low}

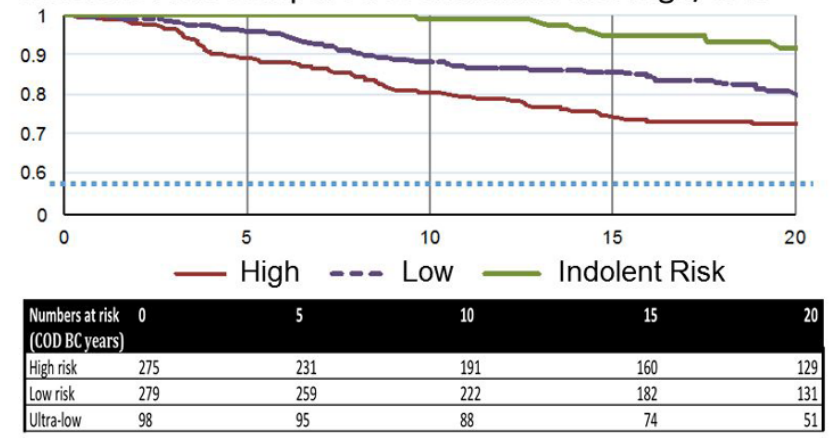

123 Comparison of Magee and OncotypeDx Recurrence Scores in Luminal-A Immunophenotype Breast Cancers

Shikha Bose, Ann E Walts. Cedars-Sinai Medical Center, Los Angeles, CA.

Background: Oncotype DX is currently used to estimate the risk of distant recurrence and predict chemotherapy benefit in estrogen receptor positive $(\mathrm{ER}+)$ breast cancer. Recent reports suggest that the Magee recurrence scores obtained using data routinely included in a pathology report can predict the OncotypeDX recurrence score (OncoRS) and obviate ordering the expensive assay in a substantial number of ER+ breast cancers. Design: Tumor size, Nottingham score, and ER, PR, Ki67, and Her 2 information were extracted from pathology reports of 128 consecutive ER+ invasive breast cancers with $\mathrm{Ki} 67<14 \%$ and available OncoRS. Data were entered into the automatic RS calculator accessible at the Univ. of Pittsburgh Dept. of Pathology website to obtain the recurrence scores for Magee equations \#1-\#3 (Klein et al. Mod Pathol 2012). Since all of our cases were evaluated for Ki67, the average of Magee equations \#1 and \#3 was calculated as the "average modified Magee recurrence score" (AMM RS). The AMM RS and OncoRS were compared per tumor.

Results: The median age at lumpectomy was 53 years (24-81 yrs) and the median tumor size was $1.5 \mathrm{~cm}(0.4-9.0 \mathrm{~cm})$. The OncoRS were 71 low-risk (LR), 52 intermediate-risk (IntR), and 5 high-risk (HR). The AMM RS were $77 \mathrm{LR}, 48$ IntR, and $3 \mathrm{HR}$. The table shows the distribution and concordance of OncoRS and AMM RS. 90 (70\%) tumors had concordant scores. Among the 38 tumors with discordant scores there were no LR/ HR discordances. All of the 3 HR AMM RS tumors were HR OncoRS.

Comparison of AMM RS and OncoRS ( $N=128$ )

\begin{tabular}{|l|c|c|c|}
\hline AMM RS & Low-risk OncoRS & Intermediate-risk OncoRS & High-risk OncoRS \\
\hline & & & \\
\hline Lowf-risk $(\mathbf{n}=77)$ & 56 & 21 & 0 \\
\hline & & & 2 \\
\hline Intermediate-risk ( $=48)$ & 15 & 31 & \\
\hline & & 0 & 3 \\
\hline High-risk $(\mathbf{n}=3)$ & 0 & 3 & \\
\hline
\end{tabular}

Conclusions: OncoRS and AMM RS were concordant in $70 \%$ of our study cohort. None of the 128 tumors showed a 2 -step (LR/HR) discordance. Given that recurrence scores impact treatment, the high percentages of tumors with IntR scores $(37.5 \%$ with AMM RS and $40.6 \%$ with OncoRS) is an important limitation of both recurrence scoring algorithms. All 3 of the tumors with HR AMM RS also had HR OncoRS. Larger prospective studies with long-term follow-up are needed to determine if OncoRS testing is unnecessary for HR AMM RS tumors.

124 GATA3 Expression Is Correlated with AR and ER Expression and Negatively Correlated with Nuclear Grade in Mammary Carcinomas

Agedi Boto, Rita Abi Raad, Sara Rutter, Lori Charette, Malini Harigopal. Yale New Haven Hospital, New Haven, CT.

Background: GATA3 is a transcription factor that plays a role in multiple lines of tissue differentiation. Immunohistochemically, it is used as a marker for breast and urothelial cancer due to its relative specificity and sensitivity for these tissues over normal tissues. In breast cancer, its expression has been correlated with ER expression and responsiveness to hormonal therapy in the past. However, no studies have examined correlations between GATA3 and AR to our knowledge.

Design: We assessed GATA3, ER, PR, AR, GCDFP15, mammaglobin, and Her2 expression by immunohistochemistry in breast carcinomas of various histologic types using a tissue microarray approach. GATA3, AR, GCDFP15, and mammaglobin expression was scored semiquantitatively as 0,1 , or 2 , corresponding to the absence of 
staining, weak/focal staining, and strong/diffuse staining, respectively. ER, PR, and Her2 expression was scored semiquantitatively as $0,1,2$, or 3 corresponding to no staining, weak/focal staining, moderate staining, and strong diffuse staining, respectively.

Results: A total of 259 breast cancer cases were studied including 9 lobular carcinomas, 2 medullary carcinomas, 7 mucinous adenocarcinomas, and 241 ductal adenocarcinomas. We re-validated the previously reported correlations between GATA 3 and ER ( $r=0.46$ in our study) and ER and AR ( $r=0.33$ in our study). We also saw the expected correlation between GATA 3 and PR expression $(\mathrm{r}=0.38)$. The strongest correlation, however, was between GATA3 and AR ( $\mathrm{r}=0.61)$. There was no statistically significant correlation between GATA3 expression and Her2, GCDFP15, mammaglobin, or tumor size. Consistent with GATA3's association with prognostically favorable hormonally positive tumors, there was a negative correlation between GATA3 expression and nuclear grade $(\mathrm{r}=-0.2)$.

\begin{tabular}{|l|l|l|l|l|l|l|l|}
\hline & GATA3 & ER & PR & AR & Her2 & GCDFP & $\begin{array}{l}\text { Nuc } \\
\text { Grade }\end{array}$ \\
\hline GATA3 & 1.00 & & & & & & \\
\hline ER & 0.46 & 1.00 & & & & & \\
\hline PR & 0.38 & 0.34 & 1.00 & & & & \\
\hline AR & 0.61 & 0.33 & 0.34 & 1.00 & & & \\
\hline Her2 & -0.07 & -0.08 & -0.06 & -0.02 & 1.00 & & \\
\hline GCDFP & -0.05 & 0.02 & 0.00 & 0.10 & 0.02 & 1.00 & \\
\hline Nuc Grade & -0.20 & -0.18 & -0.13 & -0.08 & 0.21 & -0.07 & 1.00 \\
\hline
\end{tabular}

Conclusions: GATA3 expression is positively correlated with ER, PR, and AR expression and negatively correlated with nuclear grade in mammary carcinomas.

125 Sensitivity and Specificity of Five Immunohistochemical Markers in Distinguishing Breast Primary CK7+ Lesions from Lung Primary CK7+ Lesions

Agedi Boto, Lori Charette, Marina Baine, Ugur Ozerdem, Malini Harigopal. Yale New Haven Hospital, New Haven, CT.

Background: Breast cancer and lung cancer are two common types of cancer that can be seen in the $\mathrm{CK} 7+, \mathrm{CK} 20$ - metastatic setting. To distinguish these, stains like GCDFP15, ER, mammaglobin, GATA3, or the lung adenocarcinoma marker TTF-1 have been suggested. This study aims to resolve which test would yield the greatest diagnostic benefit in this commonly encountered scenario.

Design: We assessed GATA3, ER, GCDFP15, mammaglobin, and TTF-1 expression in breast and lung carcinomas using a tissue microarray approach. GATA3, GCDFP15, mammaglobin, and TTF-1 expression was scored semiquantitatively as 0,1 , or 2 , corresponding to the absence of staining, weak/focal staining, and strong/diffuse staining, respectively. ER in the lung was scored similarly but ER in the breast added a category of 2 for moderate staining.

Results: A total of 82 lung carcinoma and 259 breast carcinoma cases were studied. The 82 lung carcinomas contained 67 adenocarcinomas, 6 adenosquamous carcinomas, and 9 large cell carcinomas. The breast carcinomas included 9 lobular carcinomas, 2 medullary carcinomas, 7 mucinous adenocarcinomas, and 241 ductal adenocarcinomas. 4 of $82(4.9 \%)$ lung carcinomas showed some GATA3 expression while 2 of $82(2.4 \%)$ stained for mammaglobin and 5 of $82(6.1 \%)$ stained for ER. GCDFP15 was negative in all lung carcinomas. 41 of $82(50 \%)$ of lung carcinomas showed at least focal TTF-1 staining. 215 of $259(83 \%)$ breast carcinomas showed some GATA3 staining. 139 of $259(53.7 \%)$ breast carcinomas showed some ER expression. 46 of $259(17.8 \%)$ breast carcinomas showed some GCDFP-15 staining. 49 of 259 (18.9\%) breast carcinomas showed some mammaglobin staining. 0 of 259 breast carcinoma cases showed TTF-1 staining. Thus, the stain with the highest sensitivity for lung carcinoma in this study is TTF-1, correctly identifying $50 \%$ of lung carcinomas. The stain with the highest sensitivity for breast carcinoma is GATA3, labeling $83 \%$ of breast carcinomas. Although GATA3 shows far greater sensitivity for breast carcinoma, its specificity for breast carcinoma (95.1\%) is slightly less than that of TTF-1 (100\%) for lung carcinomas. Conclusions: In the setting of a metastatic CK $7+$ tumor of unknown origin, the two top stains to distinguish a breast from a lung primary are GATA3 and TTF-1. While GATA3 is more sensitive for identifying breast carcinoma than TTF-1 is for detecting lung cancer ( $83 \%$ vs $50 \%$ ), TTF-1 is slightly more specific for lung carcinoma than GATA3 is for breast carcinoma (specificity of $100 \%$ vs $95.1 \%$ )

\section{Breast Cancer in Women over 75: An Evaluation of Predictive} Markers and Aggressive Subtypes in Non Screen Detected Cancers

Tara Jane Browne, Michael W Bennett, Linda Feeley, Aoife McCarthy, Mark Corrigan, Fionnuala O'Connell. Cork University Hospital, Cork, Ireland.

Background: Breast cancer is a common disease of the elderly with incidence increasing with age. Many studies site increased comorbidities, higher stage at diagnosis and less effective treatment as the reasons for poorer survival rates. The aim of our study was to evaluate poor prognostic parameters including histologic grade and predictive markers in elderly women with invasive breast cancer.

Design: Patients aged 75 years and over at the time of diagnostic breast core needle biopsy for invasive carcinoma were identified from the institutional files of a tertiary referral subspecialised combined symptomatic and screening breast service over a 5 year period (2010-2014). All core needle biopsies were taken from the primary breast cancer and processed in a single laboratory with standardized uniform pre-analytics. Hormone and HER2 evaluation with immunohistochemistry and Brightfield dual in situ studies were performed as per CAP Guidelines.

Results: 1603 patients with non screen detected cancers were diagnosed over a 5 year period. $374 / 1603(23.3 \%)$ were 75 years or over at the time of diagnosis. 348/374
(93\%) had invasive carcinoma. Age range was 75-99 years, mean age of 81.9 years. Histologic subtypes; $76.2 \%$ invasive ductal carcinoma, $18.7 \%$ lobular carcinoma, $2.3 \%$ mucinous carcinoma, $1.1 \%$ papillary carcinoma, $0.9 \%$ mixed ductal and lobular carcinoma, $0.4 \%$ micro-papillary carcinoma and $0.4 \%$ mixed tubular and cribriform carcinoma. Histologic grades; $30.7 \%$ grade $3,58 \%$ grade $2,11.3 \%$ grade 1 . HER 2 profile; $86.3 \%$ HER2 negative, $13.6 \%$ HER2 positive. Triple negative tumours; $9.2 \%$. In total, $36.8 \%$ tumours were either grade 3 , HER 2 positive or triple negative. Conclusions: Our study showed that over one third (36.8\%) of women with symptomatic breast cancer diagnosed over the age of 75 had poor prognostic parameters including grade 3, HER2 positive or triple negative tumors.

127 When Pathological and Radiological Correlation Is Achieved Excision of Fibroadenoma with Lobular Neoplasia on Core Biopsy Is Not Warranted

Tara Jane Browne, Cassie Fives, Michael W Bennett, Linda Feeley, Rose Murphy, Mark Corrigan, Fionnuala O'Connell. Cork University Hospital, Cork, Ireland.

Background: Diagnosis and management of lobular carcinoma in situ (LCIS) and atypical lobular hyperplasia (ALH) remains controversial. Management options include excision biopsy, clinical and radiologic follow up or endocrine chemoprophylaxis. One area which lacks consensus guidelines is the optimum management of LCIS and ALH as the highest risk lesion diagnosed on core needle biopsy (CNB) particularily when associated with a radiologic mass. Aim of the study was to evaluate whether excision biopsy is warranted in cases of lobular neoplasia associated with fibroadenoma (FA) on CNB when the imaging target is a mass concordant with a FA.

Design: The pathology database at a single large academic centre with a combined screening and symptomatic, breast subspecialty service was reviewed for all CNBs over a 3year period with a diagnosis of FA. All cases with FA associated with ALH or classical LCIS were identified. Cases with coexistent DCIS, invasive carcinoma, papilloma, radial scar, atypical ductal hyperplasia or flat epithelial atypia and non classical LCIS were excluded as were cases where the radiologic target was discordant with a FA.

Results: 2878 consecutive radiologically guided CNB with a diagnosis of FA were identified. 21 cases met the selection criteria of concomitant ALH or classical LCIS. All cases underwent excision biopsy. Mean age 53 years (range 41-70y). CNB diagnosis was LCIS and FA in 16 cases and ALH and FA in 5 cases. Average size of fibroadenoma was $1.9 \mathrm{~cm}$ (range $0.5-2.4 \mathrm{~cm}$ ). 16 cases had residual LCIS or ALH on excision. One of the twenty-one cases ( $4.8 \%$ ) was upgraded on excision to invasive ductal carcinoma, Grade 2, $0.2 \mathrm{~cm}$ in dimension.

Conclusions: Our study includes a large number of sequential FA and reflects the rarity of FA associated with classical LCIS/ALH on CNB. To date it appears to be the only study specifically addressing radiologically concordant cases with a diagnosis of classical LCIS/ALH and FA on CNB.

We conclude that when strict pathological and radiological correlation is achieved excision of FA with LCIS/ALH on core biopsy where the imaging target is a mass $\mathrm{c} / \mathrm{w}$ a FA excision may not be required. This of course needs to be in the setting of close follow up and the understanding that 1) carcinoma that may have been seen at the time of excision is likely small and incidental and 2) that excision does not preclude the patient from developing carcinoma at this site or at any site elsewhere in the breast

\section{Is Excision Biopsy of Fibroadenomas Based Soley on Size Criteria} Warranted?

Tara Jane Browne, Michael W Bennett, Linda Feeley, Mark Corrigan, Fionnuala O'Connell. Cork University Hospital, Cork, Ireland.

Background: Fibroadenomas (FA) are the most common benign tumour in the female breast and arise in approximately $25 \%$ of asymptmatic women. The majority of FAs have classical clinical and radiologic findings and the diagnosis can be readily confirmed by core needle biospy (CNB). Most FA are managed conservatively provided there is radiologic concordance with the histologic findings. Conversely surgical excision is typically recommended for cellular fibroepithelial lesions to exclude a diagnosis of phyllodes tumor. Some studies have suggested surgical excision in all $\mathrm{FA}>3 \mathrm{~cm}$ to reduce CNB sampling errors. The aim of our study was to evaluated if surgical excision was warranted based on size criteria alone.

Design: The pathology data base at a large academic center with combined screening and symptomatic breast subspeciality service was reviewed for all CNBs with a diagnosis of FA that had a subsequent surgical excision at our institution over a 3 year period. Patient demographics including patient age was recorded, CNB diagnosis, excision diagnosis and preoperative radiologic size of FA.

Results: 2878 consecutive radiologically guided CNB with a diagnosis of FA were identified. 160 cases went on to have surgical excision at our institution. Average age 34.4years. Atypical features were reported in 38/160 CNB (23.8\%) including lobular neoplasia $(n=16)$, atypical ductal hyperplasia $(n=2)$ and cellular fibroepithelial lesion $(n=20)$. The remaining cases were reported as FAs on CNB. Average preoperative radiologic size was $26 \mathrm{~cm}$ (range $0.5-6.8 \mathrm{~cm}$ ) with over $50 \%$ of the cases $(n=81)$ measuring $>/=3 \mathrm{~cm}$. Only 1 of the 122 cases with a diagnosis of FA without atypia turned out to be a low grade phyllodes tumour on excision. The lesion in this case measured $3.4 \mathrm{~cm}$ and showed marked histologic heterogenity on excision with areas resembling a hyalinized FA.

Conclusions: Our study although relaively small suggests that surgical excision based soley on size is not warranted in radiologically concordant cases with a diagnosis of FA on CNB. 
129 Ductal Carcinoma In Situ (DCIS) -Invasive Carcinoma Risk Prediction Model (DRPM): A Novel Biomarker Model to Predict Local Recurrence in Large Cohort of Women Diagnosed with DCIS and $>15 \mathrm{yr}$ Follow Up

Gautam Bulusu, Adam Brufsky, Jennifer Picarsic, Gretchen Ahrendt, Gloria Carter, Mamatha Chivukula. Mills-Peninsula Health Services (a Sutter Health Affiliate); Dorothy Schneider Breast Cancer Center, Burlingame, CA; Univeristy of Pittsburgh, Magee Women's Hospital, Pittsburgh, PA; University of Pittsbrugh, Magee Womnen's Hospital, Pittsburgh, PA.

Background: Ductal carcinoma in situ (DCIS), a pre-invasive breast carcinoma incidence is on raise since the onset of screening mammography. Unlike invasive breast carcinoma (IC), DCIS is heterogeneous without clear prognostic indicators for recurrence, defined as either recurrent DCIS or IC. Tumor heterogeneity in DCIS makes it difficult to stratify into a single subtype. Very few prognostic tests like Oncotype DX breast cancer assay, shown to selectively predict recurrence in ER+ low-grade DCIS patients. The aim of our study was to develop a risk prediction model suitable for all subtypes of DCIS patients.

Design: Two hundred and ninety-one $(\mathrm{n}=291)$ cases of DCIS were retrieved from our Pathology database with follow-up period from 1988-2009. Paraffin blocks containing sufficient tissue involved by DCIS were selected. A set of biomarkers was used in the prediction model along with clinical and pathological factors.Statistical analysis: We identified risk factors and logically clustered to simplify probability elicitation. Clusters included factos such as estrogen exposure, biopsy histology and biomarker expression etc. Conditional probabilities using Bayesian networks that affect risk estimation, and graphical structures networks were derieved. Prediction model was built to observe the mpact of these variables through four different outputs of recurrence vs non-recurrence. Results: A total of 219 cases with complete data were evaluated. Cases with recurrence $(n=26)$ and without $(n=193)$ were evaluated. Our initial results showed that DRPM could improve the recurrence prediction sensitivity, while keeping high accuracy and specificity.

Conclusions: Our study compared a set of novel biomarkers and other important clinical variables in a novel risk prediction model (DRPM) to determine which factors can predict the recurrence in DCIS. Our initial observations of DCIS and invasive IC risk, complements traditional clinical and pathologic factors, and provide a new clinical tool to improve selecting individualized treatment for women with DCIS.

130 HER2 Immunohistochemical and Fluorescence In Situ Hybridization Discordances in Invasive Carcinoma with Micropapillary Features

Justin Caron, Erinn Downs-Kelly, H Evin Gulbahce, Rachel E Factor. University of Utah/ARUP, Salt Lake City, UT.

Background: The 2013 ASCO/CAP HER2 guidelines have a key recommendation for pathologists with respect to invasive micropapillary carcinoma (MPC) suggesting that HER2 immunohistochemical staining that is intense but incomplete (basolateral or $\mathrm{U}$ shaped) and would be considered $1+$ may actually be HER 2 amplified by fluorescence in situ hybridization (FISH). Thus, pathologists should consider reporting these specimens as equivocal and perform an alternative testing methodology. In part, this recommendation is based on a study by Vingiana et al 2013 Histopathology 63, 217-224, wherein the authors tested a series of 23 MPC that were considered 1+ by HercepTest and identified HER 2 amplification in 1 case (4\%). We sought to evaluate this possible discordance between IHC and FISH in a series of invasive carcinomas with micropapillary features that had both testing methodologies performed.

Design: A series of invasive carcinomas with MP features as described by the WHO as a carcinoma composed of small, hollow or morula-like clusters of cells surrounded by clear stromal spaces having both HER2 IHC (DAKO Hercept) and definitive HER2 FISH (DAKO HER2 IQFISH pharmDx and reflex when needed to a laboratory developed test FISH HER2/RAI1) were evaluated. All assays were scored according to the ASCO/CAP 2013 HER2 testing guidelines. Specifically for this study, IHC was scored regardless of MP features.

Results: Twelve cases with MP features composing anywhere from $40 \%-100 \%$ of the tumor histology were identified ( 7 core needle biopsies and 5 whole tissue slides from resections). By IHC, 5 cases were scored as $2+, 6$ were scored as $1+$ and one was scored as 0 . Overall, 6/12 (50\%) were ultimately FISH amplified; 2 cases were initially equivocal and required the reflex probe set. The HER2/CEN-17 or HER2/RAI1 ratios ranged from $2.1-2.6$ with an average ratio of 2.3. With respect to the FISH amplified cases, the corresponding IHC was $1+$ in four cases (33\%) and $2+$ in two cases $(17 \%)$. Conclusions: Although this represents a small case series, the presence of MP features warrants attention when evaluating HER2 IHC. The ASCO/CAP recommendation that this morphology may not stain in the typical pattern is highlighted as we found that $33 \%$ of cases with MP features had HER 2 staining that would otherwise be scored as 1+ were HER2 amplified by FISH, albeit low level amplification.

\section{Repeat HER-2/neu Testing on Grade 3 Invasive Breast Carcinoma} Resections

Cody Carter, Julie Jorns. University of Michigan, Ann Arbor, MI.

Background: The advent of trastuzumab therapy revolutionized the treatment of breast cancer and serves as a framework for theranostic testing for personalized cancer therapy. Recent American Society of Clinical Oncology and College of American Pathologists updated guidelines for Human Epidermal Growth Factor Receptor 2 (HER2) testing recommend retesting of HER2 if prior testing was negative and the tumor was grade 3, purportedly due to the increased proportion of HER 2 overexpression and heterogeneity in grade 3 tumors. We present a 21 month experience (1/2014-9/2015) of retesting HER2 at a large academic center.
Design: HER2 immunohistochemistry (IHC) was prospectively repeated on all consecutive resections with grade 3 histology and either prior negative or equivocal HER2 results. All equivocal IHC results were reflexed to dual probe fluorescence in situ hybridization (FISH) testing. Clinicopathological data was recorded. Resection H\&E and HER2 slides were reviewed for morphologic subtype, tumor grade, HER2 staining and heterogeneity.

Results: Of 221 grade 3 carcinomas, $169(76.5 \%)$ had a prior negative $(\mathrm{N}=164 ; 97 \%)$ or equivocal $(\mathrm{N}=5 ; 3 \%)$ HER2 result and had repeat testing on resection. Of these, 8 showed discordant HER2 results between biopsy and resection, 3 of which had final positive results that led to change in management. All 3 cases which led to the addition of Herceptin to the patient's regimen were equivocal by IHC at biopsy. Equivocal results were also seen in all $8(100 \%)$ discordant vs 43/161 (26.7\%) of non-discordant cases. No other clinicopathologic features were associated with discordance. Heterogeneity of tumor grade was more common in $\geq \mathrm{T} 2(7 / 96 ; 7.3 \%)$ vs $\mathrm{T} 1(3 / 73 ; 4.1 \%)$ tumors and heterogeneity in histology $(3 / 128 ; 2.3 \%$ vs $4 / 31 ; 12.9 \%)$, grade $(6 / 128 ; 4.7 \%$ vs $3 / 31 ; 9.7 \%)$ and HER2 staining $(16 / 127 ; 12.6 \%$ vs $5 / 31 ; 16.1 \%)$ were more common in $\geq$ N2 vs N0-1 stage. Clinicopathologic features of discordant cases are shown below.

\begin{tabular}{|c|c|c|c|c|c|c|c|c|c|c|}
\hline \begin{tabular}{|l|}
$\begin{array}{l}\text { Discordant } \\
\text { Cases }\end{array}$ \\
\end{tabular} & \begin{tabular}{|l|l|} 
Diagnosis \\
\end{tabular} & pT stage & pN stage & ER & PR & 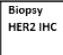 & $\begin{array}{l}\text { Biopsy HER2 FISH } \\
\text { (HER2/CEP17 ratio / } \\
\text { HER2 copyf) }\end{array}$ & \begin{tabular}{|l|} 
Resection \\
HER2 IHC
\end{tabular} & $\begin{array}{l}\text { Resection HER2 FISH } \\
\text { (HERZ/CEP17 ratio/ } \\
\text { HER2 copyy) }\end{array}$ & \begin{tabular}{|l|l}
$\begin{array}{l}\text { Trastuzumab } \\
\text { given }\end{array}$ \\
\end{tabular} \\
\hline 1 & IDC & T2 & No & Pos & \begin{tabular}{|l|l|} 
Pos \\
\end{tabular} & Neg & & \begin{tabular}{|l|l} 
Equiv \\
\end{tabular} & \begin{tabular}{|l} 
Equiv (1.85/5.8) \\
\end{tabular} & No \\
\hline 2 & IDC-1 & T1c & N1mi & Pos & Pos & Fquiv & Fquiv (1.52/3.8) & Fquiv & Neg & No \\
\hline 3 & IDC & T4 & N2a & Neg & Neg & Equiv & Neg & \begin{tabular}{|l} 
Equiv \\
\end{tabular} & ONS & No \\
\hline 14 & ILC & T4 & N2a & Pos & Pos & Equilv & Neg & Equiv & Equiv (1.71/5.3) & No \\
\hline 5 & $10 \mathrm{C}$ & T1c & N2a & Pos & Pos & \begin{tabular}{|l} 
Equiv \\
\end{tabular} & Equiv (1.71/4.2) & Neg & & No \\
\hline 6 & IDC & I1c & No & Pos & Pos & Neg & & Equiv & Pos (1.57/6.8) & Yes \\
\hline 7 & IDC & T1c & No & Neg & Neg & Nce & & Equiv & Pos (2.57/5.8) & Yes \\
\hline 8 & IDC & 12 & No & Pos & Nee & NeE & & \begin{tabular}{|l} 
Equiv \\
\end{tabular} & Pos (2.58/4.0) & Yes \\
\hline
\end{tabular}

Conclusions: Biopsy-resection HER2 discordance is uncommon and most frequently seen in cases when there is one or more equivocal result. Heterogeneity was also infrequent but most common in higher stage cancers. These findings support that repeat HER2 testing does not need to be performed on all grade 3 breast carcinoma resections, but rather on a case by case basis.

132 Clinical-Pathological Analysis of Women with Proliferative Breast Disease Undergoing Multiple Benign Biopsies: A Mayo Benign Breast Disease Cohort Study

Jodi M Carter, Derek C Radisky, Ryan D Frank, Robert A Vierkant, Stacey Winham, Marlene H Frost, Amy Degnim, Daniel $W$ Visscher. Mayo Clinic, Rochester, MN; Mayo Clinic, Jacksonville, FL.

Background: A significant proportion of women with benign proliferative breast disease at initial biopsy undergo additional subsequent biopsies for ongoing or recurring imaging or physical exam abnormalities. Changes in histopathological features over time and resulting outcomes for this subset of benign breast disease patients have not been well studied.

Design: Detailed histological analysis and overall diagnostic impression (nonproliferative disease $[\mathrm{NP}]$ vs proliferative disease $[\mathrm{PD}]$ vs atypical hyperplasia $[\mathrm{AH}]$ ) were assessed in second biopsies from 486 women with PD on initial biopsy who underwent at least two (non synchronous) breast biopsies during the years 1967-2000. Associations of changes in overall histological impression with later breast cancer risk were assessed using a multivariate proportional subdistribution model using time from second biopsy to breast cancer as the outcome. Time from index biopsy to second biopsy was included as a stratification term. Age and year of index biopsy were treated as adjustors, and death was treated as a competing event.

Results: Second biopsies were performed within five years of initial biopsy in $45 \%$, between 5 and 10 years of follow up in $25 \%$ and after 10 years of follow up in $30 \%$ of women. About half of women $(261 / 486,53.7 \%)$ retained proliferative histological changes on second biopsy, although a significant subset changed status to non-proliferative disease $(156 / 486,32.1 \%)$ or atypical hyperplasia $(69 / 486,14.2 \%)$. Breast cancer developed in 60 women overall $(12.3 \%$, overall median follow up after second biopsy $=13.5$ years). Compared to women with $\mathrm{PD}$ whose second biopsy also showed PD (i.e. no change, Ref 1.0), women with PD and found to have NP at second biopsy ( $54 \%$ of the group) had a significantly decreased hazard ratio for later breast cancer $(\mathrm{HR}=0.39,95 \% \mathrm{CI} 0.20-0.76, \mathrm{p}=0.005)$. Women with PD at first biopsy who progressed to $\mathrm{AH}$ at second biopsy (only $14 \%$ of the group) had a higher risk of breast cancer, although not statistically significant $(\mathrm{HR}=1.44,95 \% \mathrm{CI} 0.71-2.93, \mathrm{p}=0.32)$. Conclusions: Histological alterations of proliferative breast disease do not necessarily persist, and change appreciably to non-proliferative disease or conversely, to atypia in approximately half of follow-up biopsies. Changes in the histological status of breast tissue suggest a role for dynamic assessment of breast cancer risk.

133 Impact of Atypia within Multiple Non-Synchronous Biopsies on Breast Cancer Risk: A Mayo Benign Breast Disease Cohort Study

Jodi M Carter, Ryan D Frank, Robert A Vierkant, Stacey Winham, Derek C Radisky, Marlene H Frost, Ruth Kraft, Amy Degnim, Daniel Visscher. Mayo Clinic, Rochester, $\mathrm{MN}$; Mayo Clinic, Jacksonville, FL.

Background: A significant proportion of women with atypia at initial biopsy undergo subsequent biopsies for ongoing or recurring imaging or physical exam abnormalities. The impact of atypia in multiple non-synchronous benign breast biopsies on subsequent breast cancer risk has not been well studied.

Design: Retrospective histologic categorization (non-proliferative [NP], proliferative $[\mathrm{PD}]$ or atypical hyperplasia $[\mathrm{AH}]$ ) was performed in biopsies from women with $\mathrm{AH}$ index biopsies and $\geq 1$ non-synchronous biopsies. Associations between initial number of atypical foci and $\mathrm{AH}$ on subsequent biopsy were assessed by multivariate logistic 
regression with age and year of biopsy as adjustors and time between biopsies as a stratification term. Associations of histologic categories in non-synchronous biopsies with breast cancer risk were assessed using a multivariate proportional sub-distribution model (adjusted and stratified as above plus histologic category at second biopsy) using time from second biopsy to breast cancer as the outcome and death as a competing event. Results: AH occurred in index biopsies of 65/1414 (4.6\%) women with non-synchronous biopsies, compared to $5.3 \%$ of the entire cohort. Atypias comprised ADH (48\%), ALH (44\%) or both (8\%), with 1 atypical focus (per biopsy) in $54 \%, 2$ foci in $23 \%$ and $\geq 3$ foci in $23 \%$. Among 65 women with AH on index biopsy, the intervals to second biopsy were $<5 \mathrm{y}(52 \%), 5-10 \mathrm{y}(30 \%)$ and $>10 \mathrm{y}(18 \%) .35 \%(23 / 65)$ of women had AH on the second biopsy (AH/AH), while 42/65 (65\%) had NP or PD on second biopsy. Women with $\geq 3$ atypical foci on index biopsy were significantly more likely to have AH on subsequent biopsy compared to those with $\leq 2$ atypical foci (OR 7.00, 95\% CI $1.4-34.5$, $p=0.02$ ). With a mean follow-up of $15.67 \mathrm{y}$ (index biopsy) and $10.03 \mathrm{y}$ (second biopsy), breast cancer (BC) developed in $15(23 \%)$ women with AH on index biopsy. Cancers were predominantly ductal phenotype $(70 \%)$ with similar frequency in the ipsi- or contralateral breasts. By multivariate analysis, women with $\mathrm{AH}$ in multiple biopsies ( $\mathrm{AH} / \mathrm{AH}: \mathrm{N}=23$, with $8 \mathrm{BC}$ ) had a higher risk of breast cancer than women with $\mathrm{AH}$ only in their initial biopsy (AH/NP or PD: $\mathrm{N}=42$ with $7 \mathrm{BC}$ ); however, this did not achieve statistical significance $(\mathrm{HR}=1.61,95 \% \mathrm{CI} 0.49-3.99, \mathrm{p}=0.37)$.

Conclusions: Among women with multiple non-synchronous breast biopsies, multiple foci of $\mathrm{AH}$ at index biopsy is significantly associated with likelihood of $\mathrm{AH}$ on subsequent biopsy. Non-synchronous AH biopsies may confer an increased risk of subsequent cancer.

134 Adjust Cut-Off Values of Immunohistochemistry Models to Predict Risk of Distant Recurrence in Invasive Breast Carcinoma Patients

Yen-Ying Chen, Ling-Min Tseng, Ching-Fen Yang, Chih-Yi Hsu. Taipei Veterans General Hospital, Taipei City, Taiwan.

Background: Multi-gene assays are recommended for hormone receptor-positive invasive breast carcinoma to determine the risk of recurrence, but they are highly expensive. Prognostic models based on four immunohistochemical (IHC) markers (ER, PR, HER2, and Ki-67), such as IHC4 scores and Magee equations, work similarly to multi-gene assays to provide prognostic information. However, these prognostic models require adjustment and standardization before widespread use since inter-laboratory variation exists. This study aims to investigate if the prognostic values of these models can be improved by adjusting the cut-off values.

Design: Clinicopathological information of 642 consecutive cases of HER2- luminal type early breast cancer and 55 cases with multi-gene assays results was collected. The risk categories estimated by the IHC-based prognostic models were correlated to those estimated by the multi-gene assays for external references, and to the follow-up results for clinical validation.

Results: The cut-off values of the prognostic models were adjusted by the maximum positive likelihood ratio in predicting the risk categories derived from multi-gene assays in the 55 cases group. Applying the adjusted cut-off values to the cohort of 642 cases, the positive predictive values of a low risk estimate were higher than $90 \%$. The prognostic values of these models were also improved by using cut-off values refined by distant recurrence-free survival (DRFS). However, cut-off values adjusted by multi-gene assays were different from those refined by survival. Table 1 . Cut-off values and prognostic values evaluated by Harrell C index. Data presented as cut-off values (Harrell C index).

\begin{tabular}{|l|l|l|l|l|}
\hline & IHC4 score & Magee 1 & Magee 2 & Magee 3 \\
\hline Original & $\begin{array}{l}-29.9-29.9 \\
(0.7091)\end{array}$ & $\begin{array}{l}18-31 \\
(0.7428)\end{array}$ & $\begin{array}{l}18-31 \\
(0.6906)\end{array}$ & $\begin{array}{l}18-31 \\
(0.7593)\end{array}$ \\
\hline $\begin{array}{l}\text { Optimized } \\
\text { by survival }\end{array}$ & $\begin{array}{l}-19.4-49.6 \\
(0.7476)\end{array}$ & $\begin{array}{l}17.5-24.5 \\
(0.7846)\end{array}$ & $\begin{array}{l}16.3-22.2 \\
(0.7268)\end{array}$ & $\begin{array}{l}17.0-26.3 \\
(0.7619)\end{array}$ \\
\hline $\begin{array}{l}\text { Adjusted by } \\
\text { multi-gene } \\
\text { assays }\end{array}$ & $\begin{array}{l}-22.9-22.3 \\
(0.7465)\end{array}$ & $\begin{array}{l}17.1-22.5 \\
(0.7646)\end{array}$ & $\begin{array}{l}16.4-23.6 \\
(0.7094)\end{array}$ & $\begin{array}{c}16.2-21.5 \\
(0.7620)\end{array}$ \\
\hline
\end{tabular}

Conclusions: Adjusting the cut-off values of IHC-based prognostic models according to the purpose can improve their predictive values. Cut-off values adjusted by survival and multi-gene assays can be used to predict prognosis and benefit from chemotherapy, respectively.

135 HER-France - A National Database Containing 105000 Result Records of Predictive Breast Cancer Markers. Focus on HER2 Positivity Marie-Pierre Chenard, Caroline Egele, Dominique Fetique, Jean-Pierre Bellocq. Hôpitaux Universitaires de Strasbourg, Strasbourg, France; AFAQAP (Association Française d'Assurance Qualité en Anatomie et Cytologie Pathologiques), Strasbourg, France.

Background: Pathologists are at the front line in providing essential prognostic and predictive factors like HER2, ER, PR and Ki67 in invasive breast cancer. Quality of results relies on: 1) strict immunohistochemistry and/or in situ hybridization procedures, 2) systematic quality controls, 3) proficiency testing programs, and 4) collection of data allowing intra- and inter-laboratory comparison. HER-France is a web-based data collection system dedicated to these results. This tool has been developed for a local and national monitoring of these data by the AFAQAP (French national quality control association in histo- and cyto-pathology).

Design: The aim of HER-France is to provide French pathology labs with statistics on their own data compared with the national data (obtained by aggregation of data of all participating labs) to detect possible deviations from reference conditions and amend their practice if necessary.
The tool is cost-free and accessible to all French pathologists since 2011. It respects lab's anonymity and does not include any patient's personal information. Collected data include type of sample (biopsy vs lumpectomy/mastectomy), tumor type, percentage of positive tumor cells for ER, PR and Ki67, mean staining intensity for ER and PR, HER2 score and HER2 amplification status.

Results: Currently, 124 labs, both belonging to the public and private system have voluntarily joined HER-France and more than 105000 specimens have been registered. In order to avoid possible bias in the IHC/ISH results due to adverse pre-analytical conditions, focus was made on core-biopsies. Among 34178 biopsies concerning untreated primary tumors, percentage of HER2 scored $0,1,2$ and $3+$ was $49.6 \%$, $26.3 \%, 14.0 \%$ and $10.1 \%$ respectively. Among score 2 cases, $7.1 \%$ were ISH equivocal over the last year. HER2 mean positivity ( $3+$ and $2+$ amplified) was $12.13 \%$ (IC95\% [11.79-12.47]). HER2 positivity rate was $13.6 \%, 4.1 \%, 4.9 \%$ and $0.7 \%$ for NOS, lobular, mucinous and tubular carcinoma respectively. Over time, outlier labs with results that initially deviated too much, either in more or in less, from the national average came gradually closer to this average. When outlier labs were excluded, HER 2 mean positivity in France was $12.85 \%$ (IC95\% [12.23-13.47]).

Conclusions: Over time, HER-France is considered by French pathologists as an essential continually updated tool providing good practice indicators.

136 Dysregulation of the PIK3CA/AKT Pathway in Spindle Cell Metaplastic Carcinomas Arising from Papillary/Adenomyoepithelial Tumors

Esther Cheng, Timothy D'Alfonso, Yifang Liu, Sandra J Shin. Weill Cornell Medical College, New York, NY.

Background: Mutations in the PIK3CA/AKT pathway have been independently reported in both spindle cell metaplastic carcinoma (SCMC) and benign and malignant papillary tumors of the breast. In rare instances, SCMC is found arising from papillary/ adenomyoepithelial (P/AME) tumors which is evident by morphology, however, the molecular underpinnings of this relationship have not been studied. We hypothesized that dysregulation of the PIK3CA/AKT pathway is an alteration common to both tumor types which may, in part, explain this causal relationship.

Design: 33 cases of SCMC arising in a P/AME tumor were identified in our breast pathology consultation files. Of the cases with sufficient study material, immunohistochemistry using antibodies (Cell Signaling Technology) against targetable downstream mediators of the PIK3CA pathway [p110 $\alpha$ (C73F8), p110 3 (C33D4), and phospho-AKT (Ser473) as well as PTEN (138G6)] was performed using the Bond III autostainer after antigen retrieval. The SCMC and P/AME tumors were separately evaluated for each stain. A positive result was defined as $>5 \%$ of lesional cells demonstrating immunoreactivity. The SCMC and P/AME tumors of a single case were separately analyzed for PIK3CA mutations by next-generation sequencing. Results: Positive staining in both tumors for p110 $\alpha$, p110 $\beta$, phospho-AKT and PTEN was observed in $21 \%(3 / 14), 55 \%(6 / 11), 0 \%(0 / 14)$ and $71 \%(10 / 14)$ of cases, respectively. Negative staining in both components for $\mathrm{p} 110 \alpha, \mathrm{p} 110 \beta$, phospho-AKT and PTEN was observed in 64\% (9/14), 27\% (3/11), 100\% (0/14), and 7\% (1/14) cases, respectively. Results of mutational analysis of one case are pending.

Conclusions: Similar expression patterns of PIK3CA/AKT pathway proteins were commonly observed in SCMC and their associated P/AME tumors. These data provide support that these seemingly unrelated tumors share common molecular alterations in the PIK3CA/AKT pathway which, in part, may explain why SCMC arise in P/AME tumors in rare instances.

137 Subareolar Sclerosing Ductal Hyperplasia (SSDH): Further Characterization of a Distinct Clinicopathological Entity Based on Review of 35 Cases

Esther Cheng, Maria Arafah, Paula S Ginter, Timothy D'Alfonso, Syed Hoda. Weill Cornell Medical College, New York, NY; College of Medicine, King Saud University, Riyadh, Saudi Arabia.

Background: Rosen first described SSDH as a "distinct" proliferative entity without "proclivity to evolve into carcinoma" (Cancer 1987;59:1927-30). Since then, SSDH has not been further characterized.

Design: Cases diagnosed with SSDH over 15 years (2000-2015) were identified. All available clinical \& pathological material for each case was reviewed.

Results: 35 cases of SSDH were reviewed. All patients were female (age range:18-80, mean:59) with a unilateral solitary lesion (left:22, right:13) who typically presented with a mass $(31 / 35,88 \%)$, (figure 1) with mean size of $1.3 \mathrm{~cm}$ (range:0.4-3.0 $\mathrm{cm}$ ).

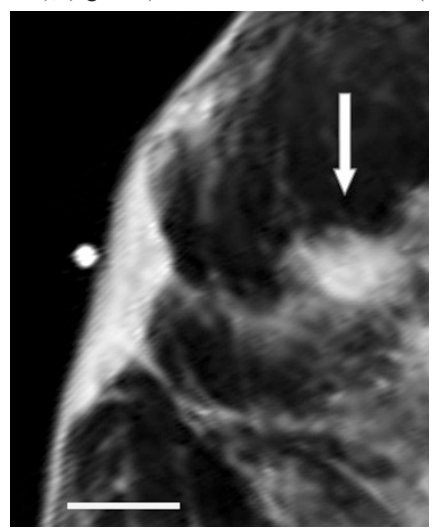


All cases showed papillary \& florid epithelial hyperplasia (diffuse), stromal proliferation (diffuse), epithelial entrapment by stroma (more at periphery).

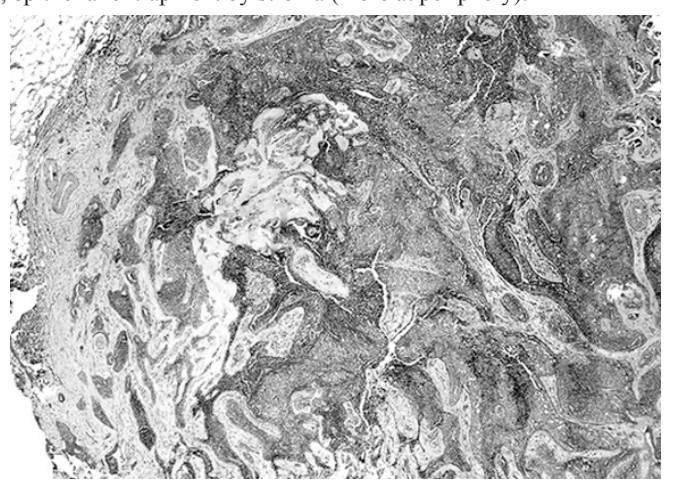

There was no involvement of nipple-areola epidermis in any case. Concurrent lesions included: low-grade adenosquamous carcinoma (n:1), DCIS (n:1), LCIS (n:1) \& ADH $(\mathrm{n}: 13)$. There was no recurrence of SSDH on follow-up of $26(75 \%)$ cases in a mean followup of 44 months (range:6-189). Subsequently occuring notable lesions included: DCIS (n:3, contralateral:1), ipsilateral ADH (n:2), ipsilateral ALH (n:1) \& "fibrocystic changes" in either breast (n:5).

Conclusions: SSDH typically occurs in older women as a central, solitary \& unilateral lesion with distinct histopathology. This benign proliferative lesion is commonly concurrent with $\mathrm{ADH}(37 \%)$, and less commonly so with carcinoma (8\%). No case recurred in this study; however, long-term follow-up is indicated as DCIS can rarely occur $(8 \%)$ in either breast.

\section{Oncotype DX Test and Modified Magee Equations: Are They}

\section{Different?}

Sachica Cheris, Jianmin Wang, Bradley Turner, Kristin Skinner, David G Hicks, Ping Tang. University of Rochester Medical Center, Rochester, NY; RTI Health Solution, Research Triangle Park, NC.

Background: Klein et al developed 3 Magee Equations (ME) that calculate Estimated Recurrence Score (ERS) with relatively high concordance with Recurrence Score (RS) of Oocotype DX test (ODX). The ERS from Modified Magee Equations (MME) developed in our institution shows good correlation with RS and is an independent prognostic factor for breast cancer (BC) patient. We investigated factors that contribute to the discordance between RS and ERS.

Design: 444 consecutive ER positive and node negative BC with both RS of ODX and ERS of MME and diagnosed between 2008 and 2014 were included. Clinical and pathology features such as patient age, tumor size, tumor grade, the expression of ER, PR, HER2 and Ki67 were documented.

Results: $69 \%$ of 444 cases had complete agreement between RS and ERS. All discordant cases were within one risk group from each other. All cases with high risk ERS also had high risk RS; $65 \%$ of high risk RS had intermediate risk ERS.

\begin{tabular}{|c|c|c|c|c|c|}
\hline & & \multicolumn{3}{|c|}{ ERS of MME } & \multirow{2}{*}{$\begin{array}{l}\text { Case } \\
\text { No. }\end{array}$} \\
\hline & & $\begin{array}{l}\text { High ERS } \\
(\%)\end{array}$ & $\begin{array}{l}\text { Intermediate ERS } \\
(\%)\end{array}$ & \begin{tabular}{|l} 
low ERS \\
$(\%)$
\end{tabular} & \\
\hline \multirow{3}{*}{$\begin{array}{l}\text { RS of } \\
\text { ODX }\end{array}$} & High RS (\%) & 35 & 65 & 0 & 34 \\
\hline & $\begin{array}{l}\text { Intermediate } \\
\text { RS (\%) }\end{array}$ & 0 & 69 & 31 & 160 \\
\hline & Low RS (\%) & 0 & 22 & 78 & 250 \\
\hline Case No. & & 12 & 186 & 246 & 444 \\
\hline
\end{tabular}

Comparing "all discordant cases" with discordant cases that with $>10$ points different between RS and ERS.

\begin{tabular}{|l|l|l|l|l|l|l|l|}
\hline & & $\begin{array}{l}\text { Case } \\
\text { No. }\end{array}$ & $\begin{array}{l}\text { HG } \\
1\end{array}$ & $\begin{array}{l}\text { ER } \\
>270\end{array}$ & $\begin{array}{l}\text { PR } \\
>270\end{array}$ & $\begin{array}{l}\text { Ki67 } \\
<15\end{array}$ & T1 \\
\hline $\begin{array}{l}\text { Low RS to } \\
\text { Intermediate ERS }\end{array}$ & all discordant cases & 56 & $40 \%$ & $92 \%$ & $50 \%$ & $68 \%$ & $75 \%$ \\
\hline & $\begin{array}{l}\text { discordance with }>10 \\
\text { points }\end{array}$ & 29 & $19 \%$ & $48 \%$ & $5 \%$ & $50 \%$ & $38 \%$ \\
\hline & & & & & & & \\
\hline $\begin{array}{l}\text { Intermediate RE to } \\
\text { Low ERS }\end{array}$ & All discordant cases & 63 & $35 \%$ & $53 \%$ & $13 \%$ & $55 \%$ & $50 \%$ \\
\hline & $\begin{array}{l}\text { Discordance with }>10 \\
\text { points }\end{array}$ & 10 & $30 \%$ & $90 \%$ & $90 \%$ & $62 \%$ & $80 \%$ \\
\hline & & & & & & & \\
\hline $\begin{array}{l}\text { High RS to } \\
\text { Intermedieate ESR }\end{array}$ & all discordant cases & 20 & $5 \%$ & $75 \%$ & $5 \%$ & $35 \%$ & $45 \%$ \\
\hline & $\begin{array}{l}\text { Discordance with }>10 \\
\text { points }\end{array}$ & 10 & $10 \%$ & $80 \%$ & $10 \%$ & $49 \%$ & $50 \%$ \\
\hline & & & & & & & \\
\hline
\end{tabular}

We found that all pathologic factors (tumor grades and size, and expression of ER, PR and Ki67) were important for the shift from Low RS to intermediate ERS; ER and PR expression and tumor size were important for the shift from intermediate RS to low ERS; and Ki67 were important for the shift from high RS to Intermediate ERS.
Conclusions: ERS accurately assigns the relative risk for most BC. Different pathologic factors are involved in the shift of between different risk subgroups.

139 Assessment of Pathologic Response and Long-Term Follow Up in Locally Advanced Breast Cancers after Neoadjuvant Chemotherapy: Comparison between Classifications

Misun Choi, Soo Youn Cho, Eun Yoon Cho. Samsung Medical Center, Seoul, Korea. Background: Neoadjuvant chemotherapy in the treatment of breast cancer is being used increasingly. Several histopathologic classification systems have been developed to assess the pathologic response of breast cancer after neoadjuvant chemotherapy (NAC). As there is a lack of consensus regarding the pathological response of postNAC specimens, reporting and interpretation is challenging. Thus, we compared seven assessment systems in relation to clinical outcome. In addition, we analyzed multiple factors associated with patient survival.

Design: We retrospectively retrieved four hundred and twenty-eight cases treated with anthracyclin with or without taxane-based regimens between 2004 and 2013 in our institute. Core biopsies before NAC and surgical sections after NAC were reviewed. National Surgical Adjuvant Breast and Bowel Project protocol B18 (NSABP-B18), Miller-Payne grading, Sataloff tumor and nodes, Chevallier's classification, Residual Cancer Burden (RCB), Residual Disease in Breast and Nodes (RDBN) and R category by AJCC were applied to assess the pathologic response of NAC. The results were correlated with survival using Kaplan-meier curves and Cox hazard regression model. Results: Mean follow-up period was 43 months. One hundred and seventeen patients (27.3\%) developed distant metastases during follow-up. Fifty-three patients (12.4\%) died of disease. Eighty-one cases (18.9\%) had complete pathologic response after NAC. All classification systems were significantly related to overall survival (OS) and disease-free survival (DFS) by univariate analysis except NSABP-B18. T-sataloff and RDBN were associated with OS by multivariate analysis ( $p=.003$ and .002 , respectively), as were Miller-Payne grading system and RDBN with DFS by multivariate analysis $(p=.009$ and $<.001$, respectively). Additionally, multivariated analysis revealed that invasive tumor size after NAC, lymphovascular invasion, and Ki-67 index after NAC were associated with $\mathrm{OS}(p=.023, .003,>.001)$, as were invasive tumor size after NAC, number of metastatic lymph node after NAC, lymphovascular invasion and Ki-67 index after NAC with DFS $(p=.030, .004,<.001,<.001$ and $<.001$, respectively).

Conclusions: RDBN index combined in post-NAC invasive tumor size, lymphovascular invasion and post-NAC Ki-67 index correlate with long-term outcome for evaluation of breast specimens after neoadjuvant chemotherapy. Incorporating these factors into routine reporting system feasible to each institutional practice could provide additional prognostic information.

\section{Micropapillary Mucinous Breast Carcinoma: An Underrecognized} Variant

Katrina Collins, Andrew Ricci. Hartford Hospital, Hartford, CT.

Background: Recent studies have described a histologic variant of mucinous breast carcinoma (MBC) that displays a combined mucinous and micropapillary pattern, i.e. micropapillary variant of mucinous carcinoma (MpVMC). Both histologic \& clinical features of this variant are incompletely characterized. Extant cases suggest a more aggressive lesion than classical MBC with greater propensity for lymphovascular invasion (LVI) and metastases to regional lymph nodes (LN). We present our institution's experience with MpVMC including clinicopathologic (CP) \& immunohistochemstry (IHC) analysis.

Design: We queried our institution's COPATH $(\mathrm{R})$ database for all MBC diagnosed over a 5-year period (2010-2015). Pathology reports and histologic slides were reviewed for cases meeting criteria for MpVMC. A representative paraffin block from each identified case was selected for $\mathrm{IHC}$ analysis.

Results: Our database screen yielded 121 cases of MBC, and 11 met histologic criteria for MpVMC, including abundant mucin in $>90 \%$ of tumor and dyshesive micropapillary "balls" of invasive carcinoma cells. CP characteristics are presented in Table 1. All cases were ER +/ve; one was HER2 -/ve. IHC for e-cadherin, MUC1 and epithelial membrane antigen highlighted small pseudoacinar tumor clusters within abundant mucin displaying an inverted growth pattern and externalized apical snouts.

\begin{tabular}{|c|c|c|c|c|c|c|}
\hline Age & Tumor size $(\mathrm{cm})$ & LVI & LN & Stage & $\mathrm{NG} / \mathrm{HG}$ & 1st Course Rx \\
\hline 31 & $0.5 / 1.3 / 1.3$ & - & $0 / 17$ & урT1с,ypN0 & III/III & NEO \\
\hline 37 & 0.53 & + & $4 / 10$ & урТ3,ypN2 & II/II & NEO \\
\hline 42 & 1.8 & + & $0 / 5$ & pT1c,pN0 & II/II & SM SLN \\
\hline 45 & 8 & - & $0 / 3$ & pT3,pN0 & II/II & TM SLN \\
\hline 64 & $0.8 / 1.2$ & - & $0 / 2$ & pT1c,pT1b(m),pN0 & $I / I$ & TM SLN \\
\hline 65 & 3.5 & - & $0 / 4$ & pT2,pN0 & $\mathrm{II} / \mathrm{II}$ & SM SLN \\
\hline 69 & 19 & - & $1 / 13$ & pT3,pN1a & I/I & TM AX \\
\hline 72 & 5 & - & $2 / 15$ & pT3,pN2a & II/II & TM AX \\
\hline 79 & Core biopsy & - & ND & N/A & III/II & $\mathrm{N} / \mathrm{A}$ \\
\hline 79 & 1.5 & - & $1 / 21$ & pT1c,pN1mi & III/III & SM AX \\
\hline 81 & 0.1 & - & ND & pT1b,pNx & $\mathrm{I} / \mathrm{I}$ & SM \\
\hline
\end{tabular}

NEO: Neoadjuvant Rx; TM/SM: Total/Segmental mastectomy; SLN: Sentinel LN; AX: Axillary LN dissection; ND: Not done

Conclusions: Classical MBC tends to affect peri- and post-menopausal women, presenting an indolent course with little LVI or LN metastases. Our 2 index cases occured in young women and were discovered after partial tumor responses to neoadjuvant 
therapy. MpVMC accounts for $<10 \%$ of the MBC diagnosed over the study period. Recognition of this variant may lead to an approach emphasizing the hormone receptor targets even perhaps in young women presenting with LN positive disease.

141 Breast Cancer Tumor Stroma Ratio and Stroma Type Are Not Associated with Hormone Receptor Status, Lymph Node Status or Tumor

\section{Grade}

Frances Compton, Yumi Kojima, Hongxia Sun, Xiaohong I Wang, Michael Covinsky, Songlin Zhang. The University of Texas Health Science Center at Houston, Houston, TX. Background: Tumor microenvironment plays a significant role during tumor development and has important prognostic and therapeutic values. Breast cancer tumor stroma ratio has been shown to correlate with cancer prognosis. Specifically, stroma-rich ( $\geq 50 \%$ stroma) early breast cancer has been associated with relapse-free and overall survival, especially in triple-negative breast cancer. The goal of the current study is to evaluate the tumor stroma ratio and tumor stroma type in relation to hormone receptor status, lymph node status and tumor grade.

Design: The study was approved by our Institutional Review Board, and 127 cases from 2009 to 2014 were selected for evaluation. One Hematoxylin and Eosin stained slide with representative tumor tissue was selected from each case and was then independently and blindly evaluated by 2 observers to determine tumor stroma ratio and tumor stroma type for each case. A third review was performed if the first 2 reviewers had differing opinions. Tumor stroma ratio was classified as stroma-rich ( $\geq 50 \%$ stroma) or stroma-poor; and the predominant stroma type was classified as fibrotic/sclerosing, desmoplastic, inflammatory, or normal breast-like. Additionally, hormone receptor status, lymph node status and tumor grade was collected from the original pathology reports for analysis.

Results: Our results showed stroma-rich tumor in 46/127 (36.2\%) cases and stroma-poor tumor in $81 / 127$ (63.8\%) cases. Results of stroma type showed that $62 / 125(49.6 \%)$ cases contained fibrotic/sclerosing stroma, 44/125 (35.2\%) cases contained desmoplastic stroma, 10/125 (8.0\%) cases contained inflammatory stroma and 9/125 (7.2\%) cases had normal breast-like stroma. Two cases of mucinous carcinoma had predominant mucinous stroma. Stroma ratio (rich or poor) was not significantly associated with tumor hormone status (ER/PR+ vs ER/PR-), tumor grade, or lymph node metastasis. The two predominant stroma types (desmoplastic and fibrotic/sclerosing) were not associated with tumor grade, and the inflammatory-type stroma (only 10 cases) was seen in both triple negative and ER/PR positive breast cancers.

Conclusions: Our study shows that while approximately one third of the breast cancer cases are stroma-rich, tumor stroma ratio shows no association with hormone receptor status, tumor grade, or lymph node status. Our findings are similar to previous literature results. Correlation with patient clinical survival and recurrence will be carried out in the future.

\section{Optimizing Intraoperative Sentinel Lymph Node Evaluation}

Margaret Compton, Emily S Reisenbichler. Vanderbilt University Medical Center, Nashville, TN.

Background: Intraoperative evaluation of sentinel lymph nodes for breast cancer identifies patients needing same-day completion axillary dissection. We previously demonstrated intraoperative touch prep has low sensitivity and results in same-day axillary dissection in patients with isolated micrometastases, a population that, per the Z0011 study, does not benefit from axillary dissection. A modified touch prep protocol incorporating gross evaluation was introduced at our institution.

Design: Under the prior standard protocol, lymph nodes were diagnosed as "negative" or "positive" for carcinoma by touch prep only. Under the modified protocol, lymph nodes were diagnosed as "negative for carcinoma" (no tumor cells on touch prep regardless of gross findings), "atypical cells, not diagnostic of carcinoma" (atypical cells on touch prep regardless of gross findings), "tumor cells present but no definitive macrometastatic disease" (tumor cells on touch prep without gross features of macrometastasis), or "tumor cells consistent with macrometastatic carcinoma" (tumor cells on touch prep AND gross features of macrometastasis). Data were collected on cases using the modified protocol for 1 year, and compared to data using the standard protocol from the prior 2 years. Results: There was no statistical difference in age, surgical procedure, tumor type, or frequency of macrometastases between cohorts. As expected, the modified protocol resulted in reduced sensitivity but achieved the desired $100 \%$ specificity. The prior protocol resulted in unnecessary same-day axillary dissection for isolated micrometastases in $4 / 15$ patients undergoing same-day dissection. With the modified protocol, all patients undergoing same-day dissection $(n=4)$, had macrometastases. A second surgery was required for completion dissection in 2.5 patients/year under the standard protocol and 3 patients/year under the modified protocol.

\begin{tabular}{|l|l|l|}
\hline & Standard Protocol $(\mathrm{n}=560)$ & Modified Protocol $(\mathrm{n}=203)$ \\
\hline Sensitivity & $60 \%$ & $38 \%$ \\
\hline Specificity & $99 \%$ & $100 \%$ \\
\hline PPV & $88 \%$ & $100 \%$ \\
\hline NPV & $96 \%$ & $96 \%$ \\
\hline
\end{tabular}

Conclusions: Despite low sensitivity of the modified protocol, no one underwent unnecessary axillary dissection and there was no increase in the number of second surgeries needed. A small but clinically significant number of patients benefited from same-day dissection. As touch prep of grossly negative lymph nodes requires substantial resources, gross examination only with reflex touch prep confirmation is now being considered for intraoperative evaluation of sentinel lymph nodes at our institution.
143 miR-139 in Breast Cancer Tumors and Serum a Surrogate Marker for Tumor Aggression

Hongyan Dai, Sarah Schmitt, Ziyan Pessetto, Fang Fan, Andrew K Godwin, Ossama Tawfik. Kansas University Medical Center, Kansas City, KS.

Background: Micro RNAs (miRNA) are non-protein coding endogenous RNAs that involve in the post-transcriptional regulation of gene expression. Many miRNAs were found to be associated with carcinogenesis and metastasis of breast cancer. Our previous data demonstrated that miRNA-139-5p (miR-139) level is differentially expressed in breast cancer. Evaluating the serum miR-139 signature profile could serve as a potential noninvasive diagnostic/prognostic marker. In this pilot study we compared and correlated miR-139 expression in paired serum and tissue samples from patients with breast cancer as a surrogate marker for tumor aggression.

Design: RNA was isolated from formalin fixed paraffin embedded (FFPE) tissue and serum samples from 18 patients diagnosed with invasive ductal carcinoma. miR-139 expression was measured by RT-qPCR in paired of matched serum and FFPE samples from patients with IDC. Each sample type (serum or FFPE) was normalized to miR-139 expression levels in a set of 10 serum or 10 FFPE normal breast tissue samples with no diagnostic abnormalities (normal) to yield a fold change in expression relative to normal samples. Tumor grade and size, estrogen receptot (ER)/progesterone receptor (PR)/Her2/Ki67 status, and patient outcome were recorded.

Results: There was significant down regulation of miR-139 expression in all FFPE tissue samples with tumor compared to normal control tissue samples. Corresponding serum samples showed down regulation of miR-139 expression in 10 of 18 samples. miR-139 expression in tissue samples was associated with aggressive pathologic and clinical features. It was down regulated in higher grade (grades I and II vs. III), ER negative, PR negative, Her 2 positive and highly proliferative tumors (Ki67 $>10 \%$ ). Additionally its expression was down regulated in larger tumors $(\geq 2 \mathrm{~cm})$ and in deceased patients. When miR-139 serum level of expression was correlated with the above parameters, down regulation correlated with only PR negative, Her $2+$ status, with highly proliferative and larger tumors and in deceased patients. There was no correlation between tumor grade or ER status and miR-139 serum expression.

Conclusions: miR-139 is differentially expressed in breast carcinomas. Its expression is significantly associated with aggressive tumor behavior and disease progression. Modulation of miRNA expression may serve as an attractive approach for treatment of aggressive and advanced breast carcinomas. The development of reliable risk assessment biomarkers will tailoring treatment options that are more effective with less undesirable side effects.

\section{Sentinel Node Metastasis in Breast Cancer: Size Matters}

Joanna Dalland, Patrick C McGrath, Reed Spaulding, Robert McDonald, Shannon Crook, Luis Samayoa. University of Kentucky, Lexington, KY.

Background: In 2007 we reported that the probability of N2 disease in breast cancer patients with sentinel node (SN) metastasis $\leq 5 \mathrm{~mm}$ was low $^{1}$. At that time our suggestion to plan the extent of axillary dissection (AD) accordingly was controversial. In view of the $\mathrm{Z} 11$ trial $^{2}$ we revisited our previous observation and confirmed with this new series that the extent of AD can be determined by the size of SN tumoral deposits.

Design: A data search from 2006 to present identified 1,202 SN breast cancer patients. Data including type of resection (mastectomy versus lumpectomy), SN status, non-SN (NSN) status, number of SN and NSN, size of tumor deposits, T stage, and N stage was collected. Thirty-seven patients with $(+)$ axilla were excluded from the final analysis (21 SN failure and 16 no tumor deposit size reported). Patients with (+) SN were then stratified into a N1b or lower and a N2a or higher categories according to the size of SN tumor deposits $\leq 5 \mathrm{~mm}$ or $>5 \mathrm{~mm}$. Results were then submitted for Chi-square statistical analysis.

Results: See tables 1 and 2 .

Table 1. Overall Nodal Stage Distribution.

\begin{tabular}{|l|l|l|l|l|l|l|}
\hline & N0 & N0(itc) & N1(mi) & N1a/N1b & N2a & N3a \\
\hline \# Patients (\%) & $973(80.9 \%)$ & $15(1.2 \%)$ & $61(5.1 \%)$ & $120(10.0 \%)$ & $26(2.2 \%)$ & $7(0.6 \%)$ \\
\hline
\end{tabular}

Table 2. Nodal Stage According to SN size of tumor deposits $\leq 5 \mathrm{~mm}$ versus $>5 \mathrm{~mm}$.

\begin{tabular}{|l|l|l|}
\hline & N1b or Lower $(\%)$ & N2a or Higher (\%) \\
\hline SN Tumor Deposit $\leq 5 \mathrm{~mm}$ & $134(97.8 \%)$ & $3(2.2 \%)$ \\
\hline SN Tumor Deposit $>5 \mathrm{~mm}$ & $34(61.8 \%)$ & $21(38.2 \%)$ \\
\hline
\end{tabular}

Of the 3 patients with $\mathrm{SN} \leq 5 \mathrm{~mm}$ tumor deposits and $\mathrm{N} 2 \mathrm{a}-3 \mathrm{a}$ disease, 2 had a SN largest deposits reported as measuring exactly $5 \mathrm{~mm}$, of which one had a $7 \mathrm{~mm}$ largest tumor deposit in a NSN. The third patient had a $3 \mathrm{~mm}$ largest SN deposit with $5(+)$ total lymph nodes (2 SN and $3 \mathrm{NSN}$ ).

Conclusions: Irrespective of $\mathrm{T}$ stage, the probability of having N2 disease with a SN tumor deposit $\leq 5 \mathrm{~mm}$ is very low $(2.2 \%, \mathrm{p}<0.05)$. To avoid overtreatment of the axilla and in the context of Z11, AD should be based on SN tumor deposit $>5 \mathrm{~mm}$ regardless of type of resection.

${ }^{1}$ Samoilova E1, Davis JT, Hinson J, Brill YM, Cibull ML, McGrath P, Romond E, Moore A, Samayoa LM. Size of sentinel node tumor deposits and extent of axillary lymph node involvement: which breast cancer patients may benefit from less aggressive axillary dissections? Ann Surg Oncol. 2007;14(8):2221-2227.

${ }^{2}$ Giuliano AE, Hunt KK, Ballman KV, Beitsch PD, Whitworth PW, Blumencranz PW, Leitch AM, Saha S, McCall LM, Morrow M. Axillary Dissection vs No Axillary Dissection in Women With Invasive Breast Cancer and Sentinel Node Metastasis, A Randomized Clinical Trial. JAMA. 2011;305(6): 569-575. 
145 Nuclear Grading of Breast Cancer Based on Training by Examination of Estrogen-Receptor Negative Tumors: Performance with and without Incorporation into the Edinburgh Simplification of Nottingham Grade

Leslie Dalton. South Austin Medical Center, Austin, TX.

Background: Nuclear grade is the least satisfying of Nottingham component scores. Little work has been performed in trying to refine nuclear grade.

Design: The training set was an H\&E stained tissue microarray providing samples of 60 estrogen receptor negative (ER-) tumors. 25 were triple negative (TN), and 23 ER-/ PR-/HER2+. Subjective analysis defined a "very high" nuclear grade (VHG). Tumors with VHG had any two of: nucleoli obvious at intermediate microscopic power (200X), very large nuclei (estimate of $>12$ microns in diameter), or marked variation in size and shape of nuclei (VSS). Unpredictable spacing of nuclei was commonly associated with VSS. VHG was tested on 512 stage 1 cancers, 376 stage 2, and 176 stage 3 . Survival analysis evaluated VHG as a stand-alone attribute, and after incorporation into the Edinburgh simplification (ES) of the Nottingham scheme. The ES allows for high nuclear grade to promote low mitotic score (MS) tumors to a high combined grade. VHG was a conservative approach for high grade assignment in those cancers with low MS. Results: Relevant hazard ratios (HR) and p-values for each stage are shown in table 1:

\begin{tabular}{|l|l|l|l|}
\hline Attributes with hazard ratio $; \mathrm{p}$-values & Stage one & Stage two & Stage three \\
\hline Very high nuclear grade & $4.3 ; 0.00001$ & $2.9 ; 0.0001$ & $2.2 ; 0.004$ \\
\hline High mitotic score & $4.3 ; 0.00002$ & $3.0 ; 0.0004$ & $2.2 ; 0.004$ \\
\hline Edinburgh score cutpoint $>4$ & $5.0 ;<0.00001$ & $3.4 ; 0.0002$ & $3.1 ; 0.0001$ \\
\hline Edinburgh score cutpoint $>5$ & $3.6 ; 0.0002$ & $3.3 ; 0.00002$ & $2.2 ; 0.005$ \\
\hline
\end{tabular}

While VHG and high MS corresponded to similar HR there was $25 \%$ discrepancy between the two (Cramer's V $=0.44$ ). HRs increased after VHG and high MS were combined into ES (ES score $>4$ ). Statistically significant survival stratification remained at ES score $>5$. This was indicative of poor prognosis in patients having cancers with both VHG, and high MS. Despite training on ER- tumors, $47 \%$ of VHG tumors were ER+. VHG showed 18\% ER+/lowMS, 29\% ER+/highMS, 33\%TN, 15\% ER-/PR-/HER2+. High MS showed $54 \%$ ER+, 27\%TN, 12\% ER-/PR-/HER2+. Of all TN cancers, ES $>4$ was inclusive of $76 \% .87 \%$ of ER-/PR-/HER $2+$ had ES $>4$.

Conclusions: Examination of ER negative tumors was helpful in defining VHG. After VHG was integrated into the ES, VHG had enough relevancy without redundancy to improve survival prediction. While not a validation, the Edinburgh simplification shows promise in data outside of Edinburgh.

146 The Detection of IHC-/FISH- ERBB2 Non-Amplification Mutations in 5,606 Cases of Refractory and Metastatic Breast Cancer: An Emerging Opportunity for Anti-HER2 Targeted Therapies

Siddhartha Dalvi, Katherine Pinheiro, Ann B Boguniewicz, Kai Wang, Siraj M Ali, Julia A Elvin, Jo-Anne Vergilio, James Suh, Vincent A Miller, Philip J Stephens, Jeffrey S Ross. Albany Medical College, Albany, NY; Foundation Medicine Inc., Cambridge, MA. Background: $E R B B 2$ (HER2) short variants (ERBB2mut) are activating mutations in either the kinase or extracellular domains (ECD) of the $E R B B 2$ gene that can activate ERBB2 signaling in the absence of ERBB2 gene amplification (FISH-) or ERBB2 protein overexpression (IHC-). Recent case reports suggest that metastatic breast cancers (mBC) with ERBB2mut can be successfully targeted with anti-HER2 therapies. Design: DNA was extracted from 40 microns of FFPE sections from 5,606 mBC. Comprehensive genomic profiling (CGP) was performed using a hybrid-capture, adaptor ligation based next generation sequencing assay to a mean coverage depth of $>600 \mathrm{X}$. The results were analyzed for short variant (SV) genomic alterations (GA) consisting of base substitutions, insertions and deletions and select rearrangements as well as for copy number changes (amplifications).

Results: $698(12.5 \%)$ of 5,605 mBC featured ERBB2 GA. $596(10.6 \%)$ featured $E R B B 2$ amplifications (ERBB2amp) and $137(2.4 \%)$ featured $E R B B 2$ mut. $35(0.6 \%)$ of total $\mathrm{mBC}$ had both $E R B B 2 \mathrm{amp}$ and $E R B B 2 \mathrm{mut}$, which accounted for $5.0 \%$ of all $E R B B 2$ altered $\mathrm{mBC}$. The 137 ERBB2mut $\mathrm{mBC}$ cases had a median age of 61 years (range 29 to 93 years). Samples utilized for CGP included $52(38 \%)$ from the patient's primary BC and $85(62 \%)$ from metastatic sites including bone/soft tissue/skin (12\%), liver $(20 \%)$, LN (14\%), serous cavities (6\%), lung (4\%) and miscellaneous sites $(6 \%)$. $71(52 \%) \mathrm{mBC}$ were submitted as carcinoma NOS, $44(32 \%)$ as invasive ductal, 22 $(16 \%)$ as invasive lobular and $1(1 \%)$ as mucinous carcinomas. Of the 137 ERBB2mut cases, $8(6 \%)$ featured more than 1 ERBB2mut. There were $124(85 \%)$ ERBB2 kinase domain mutations and $15(10 \%)$ extra-cellular domain ERBB2mut. The most common genes co-altered in ERBB2mut mBC were TP53 (49\%), PIK3CA (42\%), CDH1 (37\%), $M Y C(17 \%)$, and CCND1 (16\%). The enrichment of ERBB2mut in CDH1 mut mBR was significant $(\mathrm{p}=0.0006)$ and associated with relapsed lobular $\mathrm{mBC}$. Multiple case examples of kinase domain and extra-cellular domain $E R B B 2$ mut $\mathrm{mBC}$ responding to a variety of anti-HER 2 targeted therapies will be presented.

Conclusions: $E R B B 2$ mut are responsible for nearly $20 \%$ of $E R B B 2 \mathrm{GA}$ in $\mathrm{mBC}$ thus impacting thousands of patients in the US. ERBB2mut represent an emerging opportunity for targeted therapy especially for the relapsed lobular and triple negative cancers (IHC/ FISH tested ony). Further development of clinical trials exploiting the detection of $E R B B 2$ mut in $\mathrm{mBC}$ appear warranted.
147 BRCA1 and BRCA2-Associated Breast Cancers Demonstrate Distinct Histopathologic Features, Biomarker Expression Patterns and Molecular Subtypes

Constance Albarracin, Sagar Dhamne, Banu Arun, Kristin Ward, Yun Wu, Daniel G Rosen, Xiaoyan Cui, Erika Resetkova, Xiuzhen Duan, Hui Chen, Isabelle Bedrosian, Angelica Gutierrez Barrera. MD Anderson, Houston, TX; Loyola University, Chicago, IL; Baylor College Medicine, Houston, TX; University Texas, El Paso, TX.

Background: Hereditary breast cancers constitute $5-10 \%$ of all breast cancers, of which more than $50 \%$ are due to known mutations in breast cancer susceptibility genes, BRCA1 and BRCA2. Studies have shown that BRCA1 and BRCA2 associated tumors differ clinicopathological. As the BRCA-associated breast cancers continue to be characterized, studies addressing protein and molecular biomarker expression to find novel therapeutic targets in mutation associated breast cancers, are still limited. The present study was undertaken 1) to compare histopathologic features and expression of classic biomarkers in these tumors, and 2) to compare expression of potential novel markers and therapeutic targets in BRCA1, BRCA2, nonBRCA breast cancers.

Design: High risk patients undergoing genetic testing were identified from the institutional database. Breast cancers cases not fulfilling the criteria for genetic testing, were identified and served as sporadic controls. Tissue microarrays were prepared and immunohistochemical stained for ER, PR, HER2, Ki67, CK5/6, EGFR, SOX9, AR, and Annexin A1 (ANXA1). Fischer exact and chi-square tests were used for statistical analysis. A total of 164 patients were included, comprising of high risk group tested for BRCA mutation (30 BRCA1, 31 BRCA2, 34 BRCA neg), and 69 sporadic breast cancers with no family history.

Results: Majority of the BRCA1 breast cancers are grade $3(87 \%)$ and more than half belong to the triple negative (TN) $(57 \%)$ group. Compared to BRCA2 tumors, BRCA1 tumors are likely to be negative for $\mathrm{ER} / \mathrm{PR}(\mathrm{p}=0.003)$ and $\mathrm{AR}(\mathrm{p}=0.03)$; with high expression of SOX9 $(p=0.02), C K 5 / 6(p=0.02)$ and ANXA1 $(p=0.06)$. Majority of the BRCA2 cancers were also grade $3(65 \%)$ but luminal B subtype (48\%), and had the highest Ki67 index $(\mathrm{p}=0.03)$. EGFR expression did not show any statistical significance. BRCA negative tumors show features not statistically differing from the sporadic group. Conclusions: BRCA1 and BRCA2-associated breast cancers demonstrated distinct histopathologic features, specific patterns of protein expression and molecular subtypes. BRCA1-associated breast cancers are likely to be TN, grade 3, with high SOX9, CK5/6 and ANXA1 expression and loss of ER and AR compared to BRCA2 cancers. BRCA2 cancers show features intermediate to BRCA1, and BRCA negative and sporadic cancers. BRCA negative cancers from our high risk group of patients revealed characteristics similar to sporadic breast cancers.

148 Detection of Gene Amplifications in Invasive Breast Cancers by SNP-based Chromosomal Microarray

Jessica L Dillon, Jonathan Marotti, Joel Lefferts, Laura J Tafe. Dartmouth-Hitchcock Medical Center, Lebanon, $\mathrm{NH}$.

Background: Detection of biomarkers beyond hormone receptor (HR) expression and HER2 amplification in breast cancer is desirable as more targeted therapies and clinical trials become available. SNP-based chromosomal microarray can provide a whole genome overview of a tumor's chromosomal alterations including gene amplifications. Design: We selected 17 invasive breast cancers from our pathology archives. Two cases were invasive lobular carcinoma (ILC) with pleomorphic features and 15 invasive ductal carcinomas. Tumors were grouped based on HR expression and HER2 amplification status by fluorescence in situ hybridization (FISH): HR+ /HER2- ( $\mathrm{n}=9$ ), HR-/HER2$(\mathrm{n}=5)$, and HR+/HER2 $+(\mathrm{n}=3)$. FFPE tissues were macrodissected from 8 unstained, 4 micron slides and DNA extraction was performed with the QIAamp DNA FFPE Tissue Kit (Qiagen). The DNA samples were processed using the Affymetrix OncoScan FFPE Array (Affymetrix) according to the manufacturer's protocol. Nexus Express (BioDiscovery) and Affymetrix software packages were used for analysis. We defined gene amplification as a cut-off of 6.0 for high copy number gains.

Results: HER2 (17q12) amplification was confirmed in the three cases, two of which showed co-amplification of the centromeric region of chromosome 17, confirming FISH findings. One of these also showed amplification of MYC (8q24). FGFRI (8p11) amplification (copy number: 6-17) was identified in three cases, all HR+/HER2(included one of the pleomorphic ILC cases); one of these also had MYC amplification and another had CCND1(11q13)amplification. In addition, a fourth HR+/HER2- case showed amplification of both $M Y C$ and CCND1. Of note, no EGFR amplifications were identified in this sample set. 

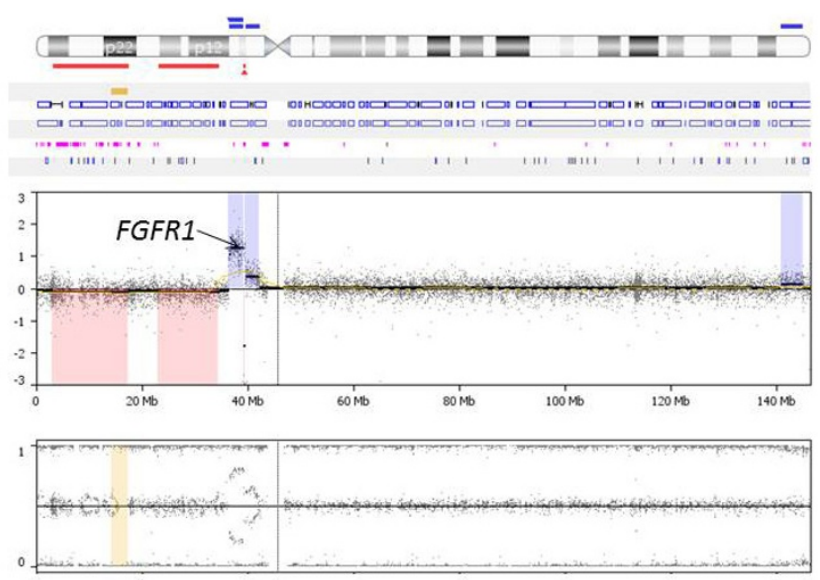

Figure 1. Chromosome 8 showing FGFR1 amplification at 8 p11.

Conclusions: In our cohort, there is a correlation with non-HER2 gene amplification and positive $\mathrm{HR}$ status (4/9 $\mathrm{HR}+$ versus $0 / 5 \mathrm{HR}-)$. Detection of $F G F R 1$ amplifications in particular may be of clinical significance as it may indicate elligibility for clinical trials for patients with FGFR alterations. Additional studies are needed to determine if breast cancers can be further subtyped based on amplification data which may inform treatment decisions.

\section{Decreased Expression of miR-26b in Advanced Breast Cancer} Qingqing Ding, Yan Wang, Zhuang Zuo, Naoto T Ueno, Wei Wei, Lei Huo. University of Texas MD Anderson Cancer Center, Houston, TX.

Background: MicroRNAs (miRNAs) are small non-coding RNAs that have been implicated in tumorigenesis, metastasis and response to treatment in cancer. Published profiling studies have identified differentially expressed miRNAs in breast cancer in general; however, data on miRNA expression in advanced breast cancer are scant. The goal of this study is to identify miRNAs as targetable markers in advanced breast cancer. Recent studies have suggested that miR-26b may act as a tumor suppressor in glioma, colorectal cancer and hepatocellular carcinoma. The role of miR-26b in advanced breast cancer has not been elucidated and is the interest of this abstract.

Design: Quantitative real-time PCR was performed using miRNA-specific primers on total RNA extracted from fresh frozen tissue. Forty-five invasive breast tumors and 11 normal breast tissue samples from 50 patients treated between 1997 and 2011 at our institution were included in the PCR analysis. The inclusion criteria were as follows: clinically and/or pathologically, T4, or tumor $\geq 5 \mathrm{~cm}$, or T2 (tumor $\geq 3 \mathrm{~cm}$ ) N2. In situ hybridization (ISH) was carried out using DIG-labeled miRNA probes in 29 formalinfixed paraffin-embedded (FFPE) invasive breast tumor samples with normal epithelium on the same slides to confirm the differential expression. The ISH signal intensities were analyzed with an intensity measurement software. Wilcoxon rank sum test and student $\mathrm{t}$-test were used for statistical analysis. Patient survival was plotted by the Kaplan-Meier method. P-values $<0.05$ were considered significant.

Results: All 45 patients were women of 27 to 98 years of age at the time of diagnosis. The clinical/pathologic stages were: IIB (2), IIIA (8), IIIB (17), IIIC (14) and IV (4). MiR-26b expression was significantly decreased in tumor compared with normal tissue by $q$ PCR analysis $(3.1$-fold, $p<0.0001)$. ISH showed that miR-26b was decreased in tumor cells compared with the normal breast epithelium on the same FFPE tissue slides (2.6-fold, $\mathrm{p}<0.0001)$. The follow-up time in our cohort of 45 cancer patients ranged from 3 months to 159 months (median, 39 month). Compared with those with higher miR-26b expression $(n=21)$, patients with lower miR-26b expression $(n=24)$ had shorter distant metastasis-free survival $(\mathrm{p}=0.022)$ and overall survival $(\mathrm{p}=0.023)$.

Conclusions: MiR-26b expression was significantly decreased in an advanced breast cancer cohort compared with normal breast tissue, and lower expression of miR26 was associated with worse survival. MiR-26b and molecules in its regulatory pathways may serve as candidate therapeutic targets in advanced breast cancer.

150 Cliniopathologic Features in Predicting Complete Pathologic Response (pCR) after Neoadjuvant Herceptin Chemotherapy in HER2+ Breast Cancers

Qingqing Ding, Elizabeth A Mittendorf, Genevieve R Lyons, Roland L Bassett, Kelly K Hunt, Aysegul A Sahin, Yun Wu. UT MD Anderson Cancer Center, Houston, TX.

Background: HER2 + breast cancer (BC) is an aggressive subtype that accounts for 15$20 \%$ of all BC. Targeted therapy with trastuzumab has improved the survival of HER $2+$ $\mathrm{BC}$ patients (pts) in the adjuvant settings. Recent studies showed that neoadjuvant therapy (NAT) with trastuzumab and anthracycline (A)/taxane (T) resulted in $40-60 \%$ complete pathologic response (pCR) in HER2+ BC. Achieving pCR after NAT is a strong indicator of therapy benefit, and an early surrogate for favorable long-term outcome. However, there are currently no models to predict pCR accurately in those pts. Previous nomograms designed to predict $\mathrm{pCR}$ in $\mathrm{BC}$ preceded the routine use of trastuzumab as part of the NAT regimen. This study aims to identify predictors for $\mathrm{pCR}$ for HER2 + BC after NAT with trastuzumab and AT.

Design: Data from 591 HER2+ pts. who received NAT with trastuzumab and AT between 2007-2012 were collected. Clinicopathologic features including age, race, clinical stage, hormonal status, tumor nuclear grade, tumor Ki67 proliferation rate, chemo-agent received and pathological response ( $\mathrm{pCR}$ vs non-pCR) were recorded. Univariate logistic regression models were used to explore the relationship between these features and $\mathrm{pCR}$.

Results: Patient (pt) age ranged from 21-87 yrs (median 51 yrs). 349 (59\%) were white, 121 (21\%) Hispanic, 83 (14\%) African-American

and $38(6 \%)$ Asian. $15(2.5 \%)$ pts. presented with clinical stage I disease, $336(57 \%)$ stage II, and $240(40.5 \%)$ stage III disease. $288(49 \%)$ pts. achieved a pCR after NAT. Clinicopathologic predictors for pCR after NAT with transtuzumab and A/T in HER2+ $\mathrm{BC}$ are summarized in Table 1.

Table 1. Clinicopathologic predictors for pCR after NAT in HER2+ BC

\begin{tabular}{|l|l|l|l|}
\hline Clinicopathologic Factors & Odds Ratio & 95\% Confidence Interval & p-value \\
\hline ER (numeric) & 0.987 & $0.983-0.991$ & $<0.0001$ \\
\hline PR (numeric) & 0.991 & $0.986-0.996$ & 0.0002 \\
\hline Ki67 (numeric) & 1.013 & $1.002-1.024$ & 0.0165 \\
\hline $\begin{array}{l}\text { Nuclear Grade } \\
\text { 2 vs 1 }\end{array}$ & 7.158 & $0.297-172.681$ & 0.0040 \\
\hline 3 vs 1 & 12.435 & $0.520-297.291$ & \\
\hline $\begin{array}{l}\text { Neoadjuvant Therapy } \\
\text { Taxol only vs. Taxol + } \\
\text { Anthracycline }\end{array}$ & 0.42 & $0.26-0.68$ & 0.0005 \\
\hline
\end{tabular}

Conclusions: Our study showed that high nuclear grade, high Ki67, and NAT with A and $\mathrm{T}$ (vs. Taxol alone) were associated with increased odds ratio of pCR while ER positivity was associated with the reduced odds of pCR. For every percentage increase of ER expression, there was an approximate $1 \%$ reduction in odds of $\mathrm{pCR}$. These results will be used to build an updated nomogram that can be used in contemporary therapy for HER2+ BC.

151 Impact of an Alternative Chromosome 17 Probe and the 2013 ASCO/CAP Guidelines on FISH for the Determination of HER2 Gene Status in Breast Cancer

Alana R Donaldson, Shashirekha Shetty, Christine Rivera, Bryce P Portier, James $R$ Cook, G T Budd, Erinn Downs-Kelly, Roger D Klein, Christopher P Lanigan, Raymond Tubbs, Benjamin C Calhoun. Cleveland Clinic, Cleveland, OH; Houston Methodist Hospital, Houston, TX; University of Utah, Salt Lake City, UT.

Background: FDA-approved dual probe $H E R 2$ FISH assays provide the ratio of HER2 to CEP17, a centromeric enumeration probe for chromosome 17. However, CEP17 copy number alterations may skew this ratio. Our goal was to analyze the use of an alternative chromosome 17 reference locus (D17S122) to accuratelyassess HER2 gene status in breast cancer.

Design: An alternative chromosome 17 reference locus (D17S122) with a stable copy number was identified using an array-based approach as previously described (Portier BP et al. J Mol Diagn 2013;15:912 abstr. ST38). Over a 30 month period, 317 specimens with suspected chromosome 17 aneusomy on HER2:CEP17 FISH underwent reflex testing with HER2:D17S122 probes.

Results: Using CEP17 and following the $2007 \mathrm{ASCO} / \mathrm{CAP}$ Guidelines, there were $50(16 \%)$ amplified, $28(9 \%)$ equivocal, and $239(75 \%)$ non-amplified cases. With CEP17 and the $2013 \mathrm{ASCO} / \mathrm{CAP}$ Guidelines, there were 70 (22\%) amplified, 74 (23\%) equivocal, and $173(55 \%)$ non-amplified cases. Reflex HER2:D17S122 FISH changed the interpretation in 86/317 (27\%) and 88/317 (28\%) of cases using the 2007 and 2013 guidelines, respectively. Following 2007 guidelines, 41/317 (13\%) were re-classified from non-amplified to amplified: $28(68 \%)$ with a mean $H E R 2$ copy number $\geq 4.0$ and $<6.0,9(22 \%)$ with a mean HER2 copy number $<4.0$, and $4(10 \%)$ with $\geq 6.0$ HER 2 on the initial HER2:CEP17 FISH. Using 2013 guidelines, 40/317 (13\%) were re-classified from equivocal to amplified, all of which had a mean $H E R 2$ copy number $>4.0$ and $<6.0$ on the initial HER2:CEP17 FISH.

Conclusions: In this study, $13 \%$ of cases were re-classified from negative to amplified under the $2007 \mathrm{ASCO} / \mathrm{CAP}$ Guidelines or from equivocal to amplified under the 2013 ASCO/CAP Guidelines. The addition of the HER2:D17S122 reflex probes resolved HER2 status in tumors with CEP17 copy number alterations. These findings underscore the 2013 Guideline recommendations for testing with an alternative chromosome 17 probe and have important implications for selecting patients for targeted therapy.

152 Impact of Chromosome 17 and HER2 Copy Number Variability on HER2 FISH in Breast Cancer Cases with CEP17 Copy Number Alterations Alana R Donaldson, Shashirekha Shetty, Christine Rivera, Bryce P Portier, James R Cook, G T Budd, Erinn Downs-Kelly, Roger D Klein, Christopher P Lanigan, Raymond Tubbs, Benjamin C Calhoun. Cleveland Clinic, Cleveland, OH; Houston Methodist Hospital, Houston, TX; University of Utah, Salt Lake City, UT.

Background: FDA-approved dual probe $H E R 2$ FISH assays provide the ratio of $H E R 2$ to $C E P 17$, a centromeric enumeration probe for chromosome 17. We analyzed HER2 and chromosome 17 copy number variability as assessed by FISH with CEP17 and an alternative chromosome 17 probe, D17S122, and the impact on determining HER2 status for the selection of patients for targeted therapy.

Design: An alternative chromosome 17 reference locus (D17S122) with a stable copy number was identified using an array-based approach as previously described (Portier BP et al. J Mol Diagn 2013;15:912 abstr. ST38). Over a 30 month period, 317 specimens with suspected chromosome 17 aneusomy on HER2:CEP17 FISH were reflexed to HER2:D17S122 FISH.

Results: Reflex HER2:D17S122 FISH changed the interpretation (using $2013 \mathrm{ASCO} /$ CAP Guidelines) in 88/317 (28\%) of cases: $17(5 \%)$ negative to equivocal; $15(5 \%)$ negative to amplified; 40 (13\%) equivocal to amplified; $9(3 \%)$ equivocal to negative; 
$2(<1 \%)$ amplified to equivocal; and $5(2 \%)$ amplified to negative. Reclassification was based on "correction" of the chromosome 17 copy number with D17S122 in 40/88 (46\%), changes in mean HER2 copy number in $38 / 88(43 \%)$, or changes in both in $10 / 88(11 \%)$. Of the 40 cases reclassified from equivocal to amplified, $33(83 \%)$ were based on "correction" of the chromosome 17 (D17S122) copy number. All 40 cases had a mean $C E P 17$ copy number $\geq 2.6$ and a mean $H E R 2$ copy number $\geq 4.0$ and $<6.0$ on initial HER2:CEP17 FISH. Of the 221/317 (70\%) cases with an average of $\geq 2.6$ CEP17 signals, $126 / 221$ (57\%) had an average of $<2.6$ D17S122 signals.

Conclusions: In a population enriched for CEP17 copy number alterations, an additional HER2:D17S122 FISH study changed the interpretation in $28 \%$ of cases. Reclassification was due to changes in chromosome 17 copy number and $H E R 2$ copy number. The utility of an alternative chromosome 17 probe (D17S122) appears to be greatest in cases with an average of $>2.6$ CEP17 signals and $>4.0$ and $<6.0$ HER 2 signals. These findings have important implications for HER 2 FISH testing and the selection of breast cancer patients for HER2-targeted therapy.

153 Isolated FEA Is Not Associated with a Significant Upgrade Rate: Correlation of Biopsy and Excision Specimens

Kathryn S Dyhdalo, Hannah Gilmore, Philip Bomeisl. University Hospitals Case Medical Center, Cleveland, OH.

Background: Flat epithelial atypia (FEA) is characterized by low-grade monomorphic nuclei replacing the normal epithelium of the terminal duct lobular unit. Although the management of these patients is controversial, many, including the majority at our institution, are referred for local excision. In an era of cost-containment and awareness of over-treatment, re-evaluating the risks and benefits of the management of this lowgrade lesion is important.

Design: All breast biopsies at our institution with a diagnosis of FEA between 1/2010 and 6/2015 were retrieved. All biopsies were signed out by a select group of breast sub-specialty pathologists. Cases with FEA representing the worst diagnosis on the biopsy were included; cases with additional findings of atypia (atypical ductal and/or lobular hyperplasia) or carcinoma (in situ or invasive) on the same biopsy, concurrent ipsilateral breast biopsy or previous ipsilateral breast biopsy (within 6 months) were excluded. Excision information, if performed, was recorded including a review of the hospital's online medical records to obtain radiologic and pathologic data. IRB approval was granted for this study.

Results: 1937 breast biopsies were performed during the study period; 245 had a diagnosis of FEA. Biopsies were excluded if they had atypia or carcinoma (156 cases), a concurrent ipsilateral biopsy with atypia or carcinoma (4 cases), or a previous ipsilateral biopsy with atypia or carcinoma ( 5 cases). 80 biopsies had isolated FEA with no previous or concurrent diagnoses of ipsilateral atypia or carcinoma; 56 of the 80 cases were excised. $33 / 56(58.9 \%)$ showed FEA and/or benign findings as the worst finding on excision, 22/56 (39.3\%) had ADH/ALH, 0/56 ( $0 \%$ ) had in situ carcinoma and $1 / 56(1.8 \%)$ had invasive carcinoma. Of note, the invasive carcinoma found on excision showed radiologic/pathologic discordance $(2.9 \mathrm{~cm}$ of calcifications on imaging, $<25 \%$ removed at biopsy), and was referred to excision regardless of the FEA diagnosis. Excluding this case due to the radiologic/pathologic discrepancy, the upgrade rate in this series is $0 / 56(0 \%)$.

Conclusions: Our series showed that the finding of isolated FEA is not associated with a significant upgrade rate, and when careful radiologic and pathologic correlation is performed, no cases were upgraded. Patients with the finding of isolated FEA after careful radiologic/pathologic correlation may be able to be monitored with clinical follow-up and imaging, rather than proceed directly to local excision.

\section{A Functional Assay for Homologous Recombination (HR) DNA} Repair Assessment in Breast Cancers

Marcia Edelweiss, Nadeem Riaz, Salvatore Piscuoglio, Charlotte KY Ng, Britta Weigelt, Robert W Mutter, Robert Delsite, Dilip Giri, Tari King, Jorge S Reis-Filho, Simon Powell. MSKCC, NY, NY.

Background: Germline mutations in the $B R C A 1$ and $B R C A 2$ genes lead to hereditary breast cancers that are defective in homologous recombination (HR) repair and sensitive to DNA damaging agents. HR deficiency (HRD) also occurs in sporadic breast cancers, but its incidence and etiology are unclear. Genomic signatures of HRD recently were employed as biomarkers in clinical trials with modest success. Recent genomics-based methods to define HRD have been shown to have excellent negative predictive value, but their positive value is limited, potentially because the HR defect that resulted in the development of the 'genomic scar' is no longer operative. We sought to develop a functional assay to define the presence of HRD at a given time in tumor development. Design: We developed an assay to assess the ability of cancer cells to localize RAD51 into sub nuclear foci in response to ex-vivo irradiation (IR) in fresh sporadic breast cancer tissue specimens from 60 patients. Positive controls, in the form of BRCA1 mutant breast cancers, were included. Cancer cells were scraped from fresh specimens at the time of grossing, were placed in RPMI solution and divided into two aliquots: one part was irradiated to $10 \mathrm{~Gy}$ and the second part was sham-treated. Following treatment, the specimens were incubated in humidified cell culture incubators at $37^{\circ} \mathrm{C}$ with $5 \% \mathrm{CO} 2$ for 4 hours, and processed for histologic and immunohistochemical analysis. RAD51 focus formation was compared between mock and IR conditions to determine relative fold induction. To rule out false negative results due to low proliferation rates, Ki67 was assessed in all cases.

Results: Seventeen of 60 (28\%) tumors displayed defective RAD51 recruitment following ex-vivo IR (RAD51-DEF). RAD51-DEF was seen in all breast cancer subtypes , including 7 of $33(21 \%)$ estrogen receptor+/HER2-, 4 of $14(29 \%)$ HER2+ and 6 of $13(48 \%)$ triple-negative cases. A bi-modal distribution of RAD51 foci was observed: defective tumors displayed negligible to no induction of RAD51 foci formation after ionizing radiation, whereas the proficient tumors displayed a $>1.75$-fold increase in the number of cells with foci.

Conclusions: We have developed a RAD51 foci formation assay following ex-vivo ionizing irradiation that may be complementary to genomics-based tests to define the subset of breast cancers lacking competent HR DNA repair and likely candidates for PARP inhibitor treatment.

155 Impact of Genetic Heterogeneity on HER2 Status with Implementation of the 2013 ASCO/CAP HER2 Reporting Guidelines

Monica V Estrada, Maria G Kuba, Brent Rexer, Suzanne Manning, Ferrin Wheeler, Ashwini Yenamandra, Melinda E Sanders. Vanderbilt University Medical Center, Nashville, TN

Background: Evidence indicates patients with HER2+ breast cancer $(\mathrm{BC})$ benefit from targeted therapies. Accurate testing for HER2 amplification/over-expression is critical to ensure that the correct patients are offered targeted treatment while patients with HER2negative tumors - who are unlikely to benefit from anti-HER2 therapy - are spared the adverse effects of these costly drugs. Guidelines for performance and reporting HER2 status, first published in 2007 by American Society of Clinical Oncology (ASCO)/ College of American Pathologist (CAP), were updated in October 2013. The new scoring criteria utilize a combination of HER2:CEP17 ratio and HER2 average copy number. Design: We retrospectively reviewed HER2 dual probe FISH results performed at our institution and/or a local reference laboratory between by $7 / 2008$ and 10/2011, classified by the 2007 guidelines. Using the existing data and the 2013 guidelines, the tumors were reclassified as follows: Amplified (AMP; HER2:CEP17 ratio $\geq 2.0$ or HER2 average copy number $\geq 6$ ), Equivocal (EQ; HER2:CEP17 ratio $<2.0$ and average HER 2 copy number $\geq 4$ but $<6$ ) or Not amplified (NA; ratio $<2$ and HER 2 average copy number $<4)$. Genetic heterogeneity $(\mathrm{GH})$ was defined as $>5-50 \%$ of scored tumors cells demonstrating HER2 amplification.

Results: Using the 2007 guidelines a total of 522 cases were scored as follows: $424(81 \%)$ NA, $15(2.9 \%)$ EQ and $83(16 \%)$ AMP. Fifty cases $(9.6 \%=50 / 522)$ changed HER2 status by the 2013 guidelines: $388(74.3 \%)$ NA, $40(7.7 \%)$ EQ and $94(18 \%)$ AMP. The new classification resulted in the following: 36 cases changed from NA to EQ, 8 cases from EQ to AMP and 3 from NA to AMP. 3 EQ cases also changed to NA. This represents a 2.75 fold increase in EQ results. GH was present in $32 \%(16 / 50)$ of cases changing HER 2 status vs. $3.8 \%(18 / 472)$ of cases not changing status. ER+ cancers were over-represented $(70 \% ; 24 / 34)$ among tumors with genetic heterogeneity.

Conclusions: The re-classification significantly increased the number of EQ cases but not AMP cases. The presence of GH was highly correlated with a change in the HER2 FISH status with application of the 2013 ASCO/CAP guidelines. Management for the newly EQ patients under standard of care guidelines would not have been altered by these results; however, subsequent correlation with outcome is likely to identify subsets of EQ tumors benefiting from therapy. The 11 patients whose status changed to AMP would have been offered anti-HER 2 therapy under the 2013 guidelines. Available follow-up information on this patient population will be subsequently reported.

156 Correlation of Protein Expression with Chromosomal Copy Number Alterations in the Progression of Early Breast Neoplasia to DCIS

Erna Forgo, Sushama Varma, Robert B West. Stanford University School of Medicine, Stanford, CA.

Background: The rise in the number of ductal carcinoma in situ (DCIS) diagnosis is contributing to concerns of over-diagnosis and subsequent over-treatment. We aimed to study the progression of breast cancer from early neoplasia to DCIS by correlating protein expression with frequent chromosomal copy number alterations (CNAs), with the ultimate goal of identifying early high-risk lesions. By studying the progression of invasive breast carcinoma at the level of breast neoplasia, we aim for the ultimate goal of improved personalized management for early breast cancer.

Design: Tissue microarrays (TMAs) of samples containing DCIS with matched early neoplasia (EN, defined as flat epithelial atypia, columnar cells change, usual ductal hyperplasia, and atypical ductal hyperplasia) were used to assess the correlation of CNAs of 8q24 (BAC clone RP11-1136L8) by fluorescence in situ hybridization (FISH), reported by The Cancer Genome Atlas to be among the most frequent CNAs in invasive breast cancer, with protein expression of C-MYC on 8q24 (Leica 1472-1) by immunohistochemistry (IHC) in both components.

Results: Previously, we found common CNAs in DCIS with concurrent invasive cancer to be closer correlated with protein expression when compared to DCIS alone $(61.2 \%$ versus $38.0 \%$ ). To expand these observations to other breast progression lesions, we studied 93 samples diagnosed as DCIS with 123 matched early neoplasia for IHC and FISH correlation for C-MYC expression. We found that the fraction of C-MYC expression positive cases was similar between DCIS and EN (57 positive of 93 total DCIS versus 124 positive of 231 total EN). However, upon stratifying the cases by C-MYC amplification, the presence of the C-MYC amplification in DCIS was associated with nearly half of the cases with positive protein expression with a sensitivity of $43.9 \%$ $(25 / 57)$ when compared to EN at $7.3 \%(9 / 124)$, and these differences were statistically significant ( $\mathrm{P}=0.0001$ by Fisher's exact test)

Conclusions: There is an increased need for risk-adapted management of DCIS and early breast neoplasia. We found additional evidence that gene specific protein expression may precede gene amplification at early stages of breast neoplasia. We aim to better understand breast cancer progression from early neoplasia to DCIS to invasive carcinoma for improving the classification of high-risk early breast cancer lesions. 
157 Increased HER2 FISH-IHC Discordance and Decreased FISH Equivocals Result from 2013 ASCO-CAP HER2 Scoring Guidelines: A Study of 11,813 Cases

Regan Fulton, Allen M Gown. PhenoPath, Seattle, WA.

Background: The 2013 ASCO-CAP HER2 breast cancer testing guidelines introduced new criteria for the designation of "amplified", "equivocal" and "nonamplified" HER2 FISH assays. In the two probe assay, "amplified" is now defined as a HER2 value of greater than or equal to 6 , or a HER2:CEP17 ratio of greater than or equal to 2 . The 2007 guidelines required a HER2:CEP17 ratio of greater than or equal to 2.2 for designation as "amplified", with no discrete value for the HER2 signal count per cell. We were interested in determining the impact of the new guidelines on HER2 IHC and FISH concordance as well as the distribution of FISH scores among the various categories. Design: We performed a retrospective review of breast cancer cases referred to our laboratory for HER2 FISH and IHC analysis by examining cases from the period of 1.5 years prior to, and 1.5 years after, the implementation of the 2013 guidelines update in our laboratory (January 2014). Our case series included 5539 and 6274 cases from the "pre" and "post" implementation periods, for a total of 11,813 cases. We compared frequencies of various categories of HER2 FISH results, as well as the HER2 IHC-FISH concordance, in the "pre" and "post" implementation periods.

Results: Implementation of the 2013 updated guidelines and definitions resulted in an increased percentage of IHC-negative ( 0 or $1+)$ cases with discordant, i.e., amplified, HER2 FISH (from $0.5 \%$ to $0.64 \%$ ). A significant fraction of these discordant cases were those with CEP17 signals/cell well below 2, resulting in a HER2:CEP17 ratio of greater than 2 even in the absence of elevated HER2 signals/cell. In the period prior to January 2014, the percentage of discordant cases with CEP17 values less than 1.8 was $17.9 \%$, while in the post 2013 guidelines period, this was $37.5 \%$. Furthermore, an overall decrease in the percentage of cases with equivocal FISH results dropped precipitously, from $2.3 \%$ to $0.9 \%$, using the new 2013 guidelines.

Conclusions: The 2013 ASCO-CAP HER2 testing guidelines for breast cancer have introduced new criteria for the definition of FISH amplification, including a group of tumors with HER2:CEP17 ratios above 2 owing to decreased CEP17 rather than increased HER2 signals/cell. These tumors would not have been called "amplified" under the 2007 guidelines, and the clinical outcome of this new subgroup is uncertain. Furthermore, our experience with a large database suggests that, unlike previous reports, the new guidelines have resulted in a significant decrease, not increase, in the percent of cases now scored as HER2 FISH equivocal.

158 PD-1/PD-L1 Expression in Brain Metastases from Breast Cancer Kara Gawelek, Nicole Williams, Vinay Varadan, Lyndsay Harris, Hannah Gilmore. Case Western Reserve University, Cleveland, OH; University Hospitals Case Medical Center, Cleveland, $\mathrm{OH}$

Background: Approximately $15 \%$ of all metastatic breast cancers (BC) lead to symptomatic brain metastases (BM). Patients with BM are difficult to treat and have a median survival of less than one year. Therefore, there is a great need to explore new therapies. More recently, targeted immune checkpoint inhibitors against programmed cell death 1 (PD-1) and programmed cell death ligand 1 (PD-L1) have been proposed as potential agents against $\mathrm{BM}$ from $\mathrm{BC}$. However, the presence of PD-1 and PD-L1 in $\mathrm{BM}$ from $\mathrm{BC}$ has not been well characterized.

Design: Tissue microarrays from a well-annotated cohort of $\mathrm{BC}$ metastatic to the brain $(n=85)$ were evaluated for PD-1 and PD-L1 expression by immunohistochemistry. Tumors were considered PD- 1 positive if staining was present in $>=10 \%$ of TILs and PD-L1 positive if there was $>=10 \%$ staining in the tumor cells and/or associated inflammatory infiltrate. Gene expression profiling was performed using FFPE material on Affymetrix HTA 2.0 arrays, and PAM50 subtypes were derived. Results of the PD-1/PD-L1 staining were correlated with ER/HER2 status and PAM50 subtype. When available, the PD-1/PD-L1 staining results were compared between the matched primary and metastatic $\mathrm{BC}$.

Results: Only 4/85 (5\%) BM showed positive staining for PD-1. When PD-1 expression was analyzed by ER/HER2 status and PAM50 subtype, PD-1 positivity was seen only the ER-HER2+ (ERBB2+) and ER-HER2- (Basal) groups. The overall frequency of PD-L1 expression was considerably higher than PD-1 and was detected in $33 / 83(40 \%)$ of all BM. PD-L1 positivity was seen in all the ER/HER2 and PAM50 subtypes. Of note, $3 / 4(75 \%)$ of the BM that were PD-1 positive were also PD-L1 positive. When looking at expression levels of both PD-1 and PD-L1 in matched cases where both the primary $\mathrm{BC}$ and $\mathrm{BM}$ were available $(\mathrm{n}=21)$, there was some gain of $\mathrm{PD}-1$ and/or PD-L1 in the metastatic tissue. No cases showed loss of PD-1 or PD-L1 expression in the BM when the primary tumor was positive.

\begin{tabular}{|l|l|l|l|l|}
\hline$\underline{\text { Marker }}$ & $\underline{\text { Neg }}=>$ Neg & $\underline{\text { Pos } \Rightarrow>\text { Pos }}$ & $\underline{\text { Neg }=>\text { Pos }}$ & $\underline{\text { Pos } \Rightarrow>\text { Neg }}$ \\
\hline PD-1 & $17(81 \%)$ & $1(5 \%)$ & $3(14 \%)$ & $0(0 \%)$ \\
\hline PD-L1 & $9(43 \%)$ & $3(14 \%)$ & $9(43 \%)$ & $0(0 \%)$ \\
\hline
\end{tabular}

Conclusions: These findings demonstrate that only $5 \%$ of brain metastases from breast cancer express PD-1, and approximately $40 \%$ express PD-L1. Because of the low frequency of PD-1 expression overall, the data suggest that targeted immune checkpoint therapies against PD-1 may not be effective in the majority of BM. PD-L1 is expressed more frequently and may be a better target, though the pathway and mechanism need to be better understood.
159 Diagnostic Terminology of Atypia on Breast Core Needle Biopsy: Correlation with Excision and Upgrade Rates

Michael George, Debra L Zynger, Gary Tozbikian. The Ohio State University, Columbus, $\mathrm{OH}$.

Background: Qualitative descriptors are sometimes used to describe atypical breast lesions. The term "focal ADH" (FADH) may be used in cases in which atypical ductal hyperplasia (ADH) is subjectively limited in extent. Focal cytologic atypia (FCA) is a descriptor that can be used if there is insufficient architectural atypia to diagnose $\mathrm{ADH}$. It is uncertain how these diagnostic modifiers impact clinical management. We conducted a retrospective study to determine the impact of terminology on excision rates of breast core needle biopsies (CBXs) with atypia and compared the upgrade rates of ADH, FADH, flat epithelial atypia (FEA) and FCA.

Design: We reviewed CBX reports from 2009-2014 with a diagnosis of ADH, FADH, FEA or FCA as the most significant finding. Excision reports were reviewed for upgrades to ductal carcinoma in situ (DCIS) or invasive carcinoma and slides from upgrade cases were reviewed. Residual atypia was noted in non-upgrades. Excision and upgrade rates were tested for correlation with diagnosis, family or personal history of breast cancer, age, mammographic target, needle size, vacuum assistance, CK5/6 use and case pathologist.

Results: We identified 390 CBXs with atypia. Diagnostic category correlated with excision rate $(\mathrm{p}=0.005)$; $\mathrm{ADH}$ had the highest and $\mathrm{FCA}$ the lowest excision rate $(84.6 \%$ vs $58.3 \%)$. FADH and $\mathrm{ADH}$ had similar upgrade rates $(11.7 \%$ vs $10.6 \%, \mathrm{p}=0.83)$, while FEA $(5.2 \%)$ and FCA $(4.8 \%)$ had lower upgrade rates. The rate of no residual atypia followed an inverse trend to the diagnostic category $(\mathrm{p}=0.08)$. Upgrade rates correlated with age $(\mathrm{p}=0.004)$ and family history $(\mathrm{p}=0.001)$, but not other variables.

\begin{tabular}{|l|l|l|l|l|l|l|}
\hline & $\begin{array}{l}\% \\
\text { Excised }\end{array}$ & $\begin{array}{l}\text { \% No } \\
\text { Residual } \\
\text { Atypia }\end{array}$ & $\begin{array}{l}\text { \% } \\
\text { Residual } \\
\text { Atypia }\end{array}$ & $\begin{array}{l}\text { \% } \\
\text { Upgrade } \\
\text { to DCIS }\end{array}$ & $\begin{array}{l}\text { \% Upgrade } \\
\text { to Invasive } \\
\text { Carcinoma }\end{array}$ & $\begin{array}{l}\text { \% Overall } \\
\text { Upgrade }\end{array}$ \\
\hline $\begin{array}{l}\text { ADH } \\
\text { n=156 }\end{array}$ & $84.6 \%$ & $30.3 \%$ & $59.1 \%$ & $8.3 \%$ & $2.3 \%$ & $10.6 \%$ \\
\hline $\begin{array}{l}\text { FADH } \\
\text { n=121 }\end{array}$ & $77.7 \%$ & $39.4 \%$ & $48.9 \%$ & $7.4 \%$ & $4.3 \%$ & $11.7 \%$ \\
\hline $\begin{array}{l}\text { FEA } \\
\text { n=77 }\end{array}$ & $74.0 \%$ & $33.3 \%$ & $61.4 \%$ & $3.5 \%$ & $1.8 \%$ & $5.2 \%$ \\
\hline $\begin{array}{l}\text { FCA } \\
\text { n=36 }\end{array}$ & $58.3 \%$ & $57.1 \%$ & $38.1 \%$ & $0 \%$ & $4.8 \%$ & $4.8 \%$ \\
\hline $\begin{array}{l}\text { All Atypia } \\
\text { n=390 }\end{array}$ & $77.9 \%$ & $35.5 \%$ & $54.9 \%$ & $6.6 \%$ & $3.0 \%$ & $9.5 \%$ \\
\hline
\end{tabular}

Conclusions: Qualitative descriptors impacted the excision rate of atypical breast lesions. While this terminology may indicate a lower rate of residual atypia, upgrades were noted in all diagnostic categories. Pathologists should use qualitative descriptors cautiously as they may prevent necessary excisions, and our data does not support excising FADH at a lower rate than $\mathrm{ADH}$.

\section{Are Microglandular Adenosis and Breast Acinic Cell Carcinomas} Related Lesions?

Felipe C Geyer, Salvatore Piscuoglio, Charlotte KY Ng, Elena Guerini-Rocco, Caterina Marchio, Zsolt Hodi, Emad Rakha, Ian O Ellis, Stuart Schnitt, Britta Weigelt, Jorge S Reis-Filho. Memorial Sloan Kettering Cancer Center, New York, NY; European Institute of Oncology, Milan, Italy; University of Turin, Turin, Italy; University of Nottingham, Nottingham, United Kingdom; Beth Israel Deaconess Medical Center and Harvard Medical School, Boston, MA

Background: Microglandular adenosis (MGA) and acinic cell carcinoma (ACC) are of triple-negative (TN) phenotype. ACC is an indolent form of invasive TN breast cancer (TNBC), whereas MGA has been shown to be a clonal and neoplastic proliferation. Both entities may give rise to high-grade TNBCs and harbor somatic genomic alterations typical of TNBCs. Due to morphologic overlap, differentiation between these lesions is challenging; hence, some have suggested that MGA and ACC constitute a spectrum of TN lesions. Here we sought to compare the genomic landscape of MGAs and ACCs. Design: DNA extracted from 4 usual and 3 atypical MGAs, 8 ACCs and matched normal tissues was subjected to targeted massively parallel sequencing (MPS) using a set of baits targeting all exons of 241 genes recurrently mutated in breast cancer and/or related to DNA repair. Somatic mutations and insertions/deletions were detected using state-of-the-art bioinformatics algorithms.

Results: MPS yielded a median coverage of $193 x$ (48x-653x), identifying a median of $7.0(1-16)$ and $6.0(2-10)$ somatic mutations per MGA and ACC, respectively. TP53 was the only highly frequently mutated gene in $\operatorname{MGAs}(5 / 7,71 \%)$ and $\operatorname{ACCs}(7 / 8,88 \%)$. All cases harboring TP53 mutations showed loss of heterozygosity (LOH) of the wild-type allele. TP53 mutations were missense (MGA 4/5; ACC 5/7) or frame-shift, and all but one located in the DNA-binding domain. Additional somatic mutations shared by both groups included those of BRCA1 (coupled with LOH), FGFR2, ERBB3, INPP $4 B$ and $P I K 3 C A$. Recurrent alterations restricted to MGA included PTEN (2/7) and MED12 (2/7) somatic mutations; whereas recurrent somatic mutations of $K M T 2 D(2 / 8), E R B B 4$ $(2 / 8)$ and $N E B(2 / 8)$ were restricted to ACCs. No significant differences between MGA and ACC were found in the overall mutation rate (Mann Whitney $U$ test, $\mathrm{p}>0.05$ ), nor in single gene comparison (Fisher's exact tests, $\mathrm{p}>0.05$ ).

Conclusions: Our findings lend further credence to the contention that MGA and ACC are part of the same spectrum of lesions, with frequent TP53 somatic mutations, and likely represent low-grade forms of $\mathrm{TN}$ disease with no/minimal metastatic potential, but with a tendency for progression to high-grade TNBCs. 
161 Loss of LINC00478 Expression Is Associated with Development of Nodal Metastasis in ER+ Breast Cance

Yesim Gokmen-Polar, Maria Zavodszky, Xiaowei Chen, Xiaoping Gu, Chinnappa Kodira, Sunil Badve. Indiana University School of Medicine, Indianapolis, IN; GE Global Research, Niskayuna, NY.

Background: Recurrence and metastasis remain a clinical challenge in ER + breast cancer. The involvement of lymph nodes is the best indicator of likelihood of distant metastases. Identification of the molecular mechanisms of underlying this process is key for prevention of metastases. Given the emerging role of long intergenic RNAs (lincRNAs) in cancer biology, we sought to identify their association in with development of nodal metastasis.

Design: Paired-end RNA sequencing (RNA-Seq) analyses was performed on matched pairs of primary and nodal metastatic tumors in patients with $E R+$ breast cancer $(n=11)$. RNA-seq data analysis pipeline, including TopHat, HTSeq and DESeq2, was used to identify lincRNAs differentially expressed in the metastatic relative to the primary tumors. qRT-PCR validation was performed using an independent cohort $(n=21$ pairs). Correlation analysis was performed between the expression of the candidate LINC00478 and known protein coding genes on the public breast cancer datasets. Gene set enrichment analysis (GSEA) and functional annotation clustering was done to determine possible functions of genes correlated with $L I N C 00478$

Results: RNA-seq analysis of matched primary and nodal mets identified 16 differently altered lincRNAs. Using a collective bioinformatics approach, we identified LINC00478 (ENSG00000215386.5) as the most significant lincRNA (FC: -2.2 fold-down; $P=0.0027$ ). Quantitative RT-PCR (qRT-PCR) further significantly validated the lower expression of LINC00478 in nodal metastasis. It is notable that LINC00478 has also been lost in most of the tumor samples as well as in lymph node metastasis. In TCGA breast cancer dataset, LINC00478 was significantly downregulated in patients with ER-positive tumors compared to uninvolved normal breast tissue. Across the ER+ and normal samples, correlation analysis with protein coding genes indicated that genes with similar expression pattern to LINC00478 were involved in cytoskeleton and/or extracellular matrix organization, or interacted with the cell membrane, and participated in regulation of cell proliferation.

Conclusions: We document for the first time that LINC00478 may play a key role in the progress of lymph nodes metastasis of ER-positive breast cancer. Its loss is associated with tumor progression and recurrence/metastasis. Novel strategies that focus on upregulation of LINC00478 might be useful for targeting breast cancer and preventing the metastases.

162 Apocrine Carcinomas Represent a Distinct, Androgen ReceptorPositive Clinicopathologic Subset of Triple-Negative Breast Cancers

Chelsea E Gottlieb, Emaculate V Tebit, Scott M Wendroth, Kristen A Atkins, Tiffany Pierson, Christiana M Brenin, Anne M Mills. UVA, Charlottesville, VA.

Background: Apocrine carcinomas (AC) comprise $0.3-0.4 \%$ of all breast cancers and are characterized by large cells with abundant eosinophilic granular cytoplasm, round nuclei, and prominent nucleoli. They are typically negative for estrogen receptor (ER) and progesterone receptor (PR) and most show no evidence of HER2 amplification. As a result, they are often clinically classified and managed as triple negative cancers (TNC). However, this subgroup may have distinct pathologic and behavioral characteristics when compared to non-apocrine triple negative cancers (NA-TNC). Androgen receptor (AR) is often expressed by AC but not NA-TNC. AR therefore represents a potential diagnostic tool and therapeutic target for AC.

Design: Our Departmental files were retrospectively queried for all AC diagnosed between 2004 and 2014. H\&E slides were reviewed and whole sections were stained with AR immunohistochemistry (AR IHC). Representative tissue microarray (TMA) cores from $27 \mathrm{NA}$-TNC (including 18 basal-like carcinomas, 7 metaplastic carcinomas, 1 carcinoma with pleomorphic lobular features and 1 carcinoma with signet ring features) were also stained for AR IHC. AR status and clinicopathologic variables were compared for the AC and NA-TNC

Results: A total of $19 \mathrm{AC}$ were identified. The grades varied (3 grade 1, 11 grade 2, and 5 grade 3 ) whereas all NA-TNC were grade 3. Mean age at diagnosis was 70 (range: 43-91) for AC and 56 (range: 29-86) for NA-TNC. 89.5\% (17/19) AC were originally diagnosed as apocrine carcinoma, while 2 were identified on morphologic review of TNC represented on the TMA. The majority (17/19) were not HER2 amplified. 19/19 AC were AR-positive and ER/PR-negative. 16/19 were strongly (3+) AR positive while $3 / 19$ were moderately $(2+)$ positive; all showed $>50 \%$ staining and $16 / 19$ showed $>80 \%$ staining. In contrast, the majority $(26 / 27)$ of NA-TNC were AR-negative. The AR-positive case showed basal-like morphology. The majority of AC presented at low stage [T1: $68.4 \%(13 / 19)$, T2: $21.1 \%(4 / 19)$, T3: $10.5 \%(2 / 19)]$, whereas NA-TNC more often presented at stage 2 or higher [T1: $48.1 \%(13 / 27)$, T2: $33.3 \%(9 / 27)$, T3: $11.1 \%(3 / 27)$, T4: $7.4 \%(2 / 27)] .31 .6 \%(6 / 19)$ AC and 29.6\% (8/27) NA-TNC had nodal metastases at presentation.

Conclusions: AC represents a clinicopathologically distinct subgroup of triple negative breast cancer characterized by AR-positivity. When compared to NA-TNC, AC more often present in older women and are lower grade and stage. However, nodal metastases are common in both groups.

163 Mass Spectrometry-Based Proteomics Identifies Lactotransferrin as Co-Localizing Protein in Amyloidosis of the Breast

Karen L Grogg, Julie A Vrana, Bolette L Caron, Jason D Theis, Paul J Kurtin. Mayo Clinic, Rochester, MN

Background: Lactotransferrin has known amyloidogenic properties, and is found in familial subepithelial corneal amyloid and seminal vesicle amyloid. While initially seen as the primary amyloidogenic protein at both sites, semenogelin is now known as the protein causing seminal vesicle amyloid. The absence of a mutation in the lactotransferrin gene in patients with familial corneal disease suggests it is a co-localizing protein. Whether lactotransferrin can serve as the sole instigating protein in amyloid fibril formation is uncertain. In our practice, we have found lactotransferrin within amyloid deposits in the breast.

Design: The Mayo Clinic LC MS/MS amyloid typing database was queried for cases in the breast. The data was screened for cases with lactotransferrin as an amyloid component. The histologic pattern of amyloid deposition was assessed by Congo red staining as well as immunohistochemistry for lactotransferrin. When possible, clinical information was obtained.

Results: Of 109 breast amyloid cases, 10 contained lactotransferrin by mass spectrometry-based proteomic analysis. 7 cases also met diagnostic thresholds for assignment of a specific amyloid type (6 AL type, 1 SAA type). In 3 cases, lactotransferrin was the only protein represented in significant amounts aside from the chaperone proteins of the amyloid proteome. These 3 patients presented with calcifications on mammography and did not have evidence of systemic amyloidosis or clonal serum immunoglobulin proteins.

The 10 cases each showed a distinct pattern of amyloid deposition, concentrically distributed around ducts and lobules. In the AL type amyloid cases, nodular deposits in the breast parenchyma were also present. Immunohistochemistry for lactotransferrin showed staining within the periglandular deposits specifically.

Conclusions: A diagnosis of amyloidosis in the breast is cause for concern as it raises the possibility of a serious systemic disease. Previous studies have shown that amyloid deposits in the breast are almost always AL type, with about half representing a localized process associated with a lymphoproliferative disorder, and the other half representing a manifestation of systemic amyloidosis. Our data shows that lactotransferrin may be incorporated into amyloid deposits at this anatomic site. In a subset of cases, lactotransferrin may be the sole instigating precursor protein. In these cases, the diagnosis of amyloid would represent a truly incidental finding of no clinical significance.

\section{Molecular Features of Breast Cancers with Triple-Negative} Phenotype among Older Women

$H$ Evin Gulbahce, Rachel E Factor, Philip Bernard, Bette J Caan, Carol Sweeney. University of Utah, SLC, UT; ARUP, SLC, UT; Kaiser Permanente Northern California, Oakland, CA.

Background: Triple-negative breast cancer (TNBC) often thought of as a feature seen among younger women. We confirmed a corresponding trend based on PCRbased molecular profiling, describing decreasing prevalence of basal-like subtypes with older age at diagnosis in a population-based breast cancer cohort (Sweeney et al. CEPB2014;23:714-24). However, TNBC do occur in older women. It is not clear whether the implications of TN immunohistochemistry(IHC) are similar in older patients.

Design: In the population-based Life After Cancer Epidemiology (LACE) breast cancer cohort, we compared molecular features of TNBC diagnosed in women ages $\leq 50,50$ 65 , and $\geq 65$. Molecular subtypes were determined using the PAM50 expression assay. Sensitivity and specificity were calculated treating clinicopathologic classification as TN from IHC as the "test" and PAM50 basal-like subtype assignment as the gold standard. We used linear regression to determine association between age and several PCRquantified molecular features: proliferation index (a summary measure of expression of 11 genes indicative of proliferative activity in the PAM50), expression of ER,PR, and ERBB2 among basal-like tumors.

Results: Based on molecular classification, the prevalence of basal-like subtype among women diagnosed $\leq 50$ years, 50-65 years, and $\geq 65$ of age were $0.16,0.09$, and 0.06 , respectively. Sensitivity of TN phenotype to correctly classify basal-like subtype was 0.91 for women $\leq 50,0.80$ for ages $50-65$, and 0.72 for women $\geq 65$. Specificity was $>0.95$ for all age groups. The trend of proliferation scores by age among tumors with basal-like subtype was inverse but did not reach statistical significance $(p=0.20)$. Among women with basal-like subtype, age was not associated with ER, PR, nor ERBB2 gene expression.

Conclusions: We found the sensitivity of TN phenotype from IHC to be an accurate classifier of basal-like subtype in all age groups, although sensitivity was lower for ages $\geq 50$. Tumors from older women with basal-like subtype did not differ from those from younger women on proliferation index, ER, PR, nor ERBB2 expression. Because of the high incidence of $\mathrm{BC} \geq 65$, the estimated $6 \%$ who have basal-like subtype are a population of interest. This analysis confirmed that basal-like tumors among women $\geq 65$ are similar to the same subtype among younger women on several molecular features.

165 HPV Detection by Chromogenic In Situ Hybridization (CISH) and p16 Immunohistochemical (IHC) Staining in Breast Cancer

Hua Guo, Juan P Idrovo, Louis J Auguste, Tawfiqul Bhuiva, Silvat Sheikh-Fayyaz. Hofstra North Shore LIJ School of Medicine, Lake Success, NY; Hofstra North Shore LIJ School of Medicine, New Hyde Park, NY.

Background: Controversy exists about the prevalence of HPV in breast tissue and its potential role in the pathogenesis of breast cancer. Most of the prior studies used PCR techniques, disadvantaged by lab contamination and the inability to the signal localization. HPV detection by CISH technology is considered more specific and added advantage of localization. There are no studies evaluating p16 IHC as a surrogate marker in breast cancer.

Design: This study is to evaluate the presence of HPV6/11 and HPV16/18 in benign and malignant breast tissue, and to assess the concordance of p16-IHC and HPV-CISH. 24 benign breast cases, 11 DCIS and 20 invasive ductal carcinoma (IDC) were studied as a 
preliminary investigation. Any nuclear staining in epithelium by CISH was considered positive. Moderately to strong cytoplasmic and/or nuclear, in a block pattern by 16 staining, was considered positive.

Results: HPV was observed in 11 of $31(35.5 \%)$ breast malignant cases by CISH.

\begin{tabular}{|l|l|l|l|}
\hline & p16 (\%) & HPV 6/11 (\%) & HPV 16/18 (\%) \\
\hline Benign breast cases & $3 / 24(12.5)$ & $2 / 18(11.1)$ & $3 / 17(17.6)$ \\
\hline Normal breast tissue adjacent to carcinoma & $1 / 30(3.33)$ & $4 / 24(16.7)$ & $4 / 22(18.2)$ \\
\hline DCIS & $2 / 11(18.2)$ & $4 / 11(36.4)$ & $5 / 11(45.5)$ \\
\hline IDC & $11 / 20(55)$ & $3 / 20(15)$ & $7 / 20(35)$ \\
\hline TNBC & $4 / 4(100)$ & $2 / 4(50)$ & $3 / 4(75)$ \\
\hline Total & $13 / 31(41.9)$ & $7 / 31(22.6)$ & $12 / 31(38.7)$ \\
\hline
\end{tabular}

The median patient age was 70 years (range $32-86$ years). Out of 31 cases, $13(41.9 \%)$, $7(22.6 \%)$ and $12(38.7 \%)$ were positive for p16 IHC, HPV6/11 and HPV16/18, respectively. 7 out of $39(17.9 \%)$ benign breast tissue were positive for high-risk HPV, including benign breast cases and normal breast tissue adjacent to carcinoma. Five out of $11(45.5 \%)$ DCIS and 7 out of 20 (35\%) IDC cases showed high-risk HPV genotype. There were 4 triple-negative breast cancer (TNBC) cases, in which $4(100 \%), 2(50 \%)$ and $3(75 \%)$ are positive for p16, HPV6/11 and HPV16/18, respectively. p16 IHC expression showed good concordance with HPV-CISH $(\mathrm{p}=0.06)$.

Conclusions: HPV DNA expression was seen in a significant number of malignant breast cases. There was higher frequency of HPV detection in TNBC. Study is being expanded to a large cohort of benign and malignant breast cases to further investigate the role of HPV in breast cancer pathogenesis. p16 appears to be a suitable surrogate marker for HPV DNA expression in breast cancer.

166 Molecular Alterations and Clinical Test Utilization in Advanced Breast Cancer - Parallel Characterization of Two Next-Generation Sequencing Panels

Grzegorz T Gurda, Kate M Serdy, David Dabbs, Rachel Jankowitz, Shannon L Puhalla, Adam Brufsky, Rohit Bhargava. Magee-Womens Hospital of University of Pittsburgh Medical Center, Pittsburgh, PA

Background: Recent advances in genomic profiling represent a new paradigm of cancer classification, with the potential to match patients unresponsive to standard treatment with targeted therapies.

Design: 48 advanced breast cancer samples underwent in-house next generation sequencing (NGS) with the AmpliSeq personalized cancer mutation panel (PCMP), whereas 33 other samples were referred for Foundation ONE testing (FO). All genomic alterations were categorized as single nucleotide variants $(S N V S)$, copy number variants (CNVS) or other (insertion, frameshift, fusion, splices site variant) and either actionable/ potentially actionable or variants of uncertain significance (VUS).

Results: Both NGS panels were used predominantly for invasive ductal carcinoma $(\sim 85 \%)$, either triple negative (TN;ER -/PR -/HER2-) or Luminal B-type (LB;ER+/PR +//HER2-/Ki67:15\%). On average, FO showed more $S N V$ s than PCMP for both TN (1.7, range $0-4$ vs. 0.87 , range $0-3$ ) and LB (1.7, range $0-3$ vs. 1.1 , range $0-2$ ). FO also detected more $C N V s$ for both TN (avg 0.5 vs. 3.2) and LB tumors (avg 0.5 vs. 2.7). All 4 subcategories of other variants $(\mathrm{n}=29)$ were detected by $\mathrm{FO}$, whereas findings for PCMP were limited $(\mathrm{n}=3)$. Overall, FO testing produced more actionable variants than PCMP (avg 5.6 vs. 1.5 ) but significantly more VUSs (avg 10.3 vs. 1.4). Among the top variants ( $\mathrm{n}>5$ cases), the same gene(s) were seen in both panels: TP53, PIK3CA, KRAS, PTEN, ERBB2/ERBB4 and FGFR1/FGF3. For genes not tested by PCMP, the top FO variants included MYC, CCND1 and other members of FGF/FGFR family. From a clinical standpoint, testing was ordered at similar rates in women of similar age and predominantly in a metastatic setting. Among all patients with actionable variants not including anti-ERBB2 (HER2), 11/33 (33\%) tested by FO and 8/32 (25\%) tested by PCMP received targeted therapy.

Conclusions: Every patient with advanced breast cancer had a unique molecular profile, with a few recurrent aberrations. The more extensive FO panel correlated with detection of more CNVs and other variants, but also more VUSs. This was less pronounced for SNVs, where the same high-prevalence cancer-related genes were seen in both panels. The majority of the tested samples were metastases and either TN or LB invasive ductal carcinomas. NGS reports were promptly reviewed, but no significant difference in utilization of targeted therapies was observed.

\section{The Impact of 2013 ASCO/CAP Guidelines on the Practice of HER2} Testing in a Reference Laboratory

Wedad Hanna, Elzbieta Slodkowska, Fang-I Lu, Guangming Han, Houman Nafisi, Sharon Nofech-Mozes. SHSC, U Toronto, Toronto, ON, Canada.

Background: The 2013 updated ASCO/CAP recommendations for HER2 testing in breast cancer (BC) differ from the previous mainly in A) definition of immunohistochemistry (IHC) score 3+; B) definition of amplification and equivocal categories for situ hybridization (ISH); C) acceptance of testing core needle biopsies (CNB). We examined the impact of the updated guidelines on HER2 testing practice and results in a reference laboratory.

Design: We compared data on HER2 tests in BC over a year before and after the new guidelines were implemented. The thresholds and definitions used in 2012 and 2014 were based on ASCO/CAP 2007 and 2013 guidelines respectively. Difference between two independent proportions was assessed using z-test.

Results: Table 1 summarizes our observations. There was $16.8 \%$ increase in overall cases tested. The proportion of HER $2+$ ve by either IHC or ISH remained constant. The proportion of CNB used increased significantly but the practice was not adopted by all the referring labs. The proportion of repeat testing doubled to $11.8 \%$.

\begin{tabular}{|c|c|c|c|}
\hline Table 1: & 2012 & 2014 & P value \\
\hline \# Cases tested & 2201 & 2558 & $\mathrm{~s}$ \\
\hline \# Tumors tested & 2278 & 2660 & $\mathrm{~s}$ \\
\hline \multicolumn{4}{|l|}{ Specimen type: } \\
\hline CNB (\%) & $713(32.3)$ & $1253(48.9)$ & $\mathrm{s}$ \\
\hline Excisions & $1337(60.7)$ & $1100(43)$ & $\mathrm{s}$ \\
\hline Other metastatic site (\%) & $151(6.9)$ & $205(8.1)$ & $\mathrm{S}$ \\
\hline HER $2+$ by any test $(\%)$ & $357(15.7)$ & $412(15.5)$ & NS \\
\hline Repeat testing in $<6$ months & $122(5.5)$ & $302(11.8)$ & $\mathrm{s}$ \\
\hline \multicolumn{4}{|l|}{ IHC (\%) } \\
\hline $3+$ & 268 & 306 & NS \\
\hline $2+(\%)$ & $576(25.3)$ & $541(20.3)$ & $\mathrm{s}$ \\
\hline $0 / 1+$ & 1434 & 1812 & $\mathrm{~s}$ \\
\hline Overall cases tested by ISH ${ }^{*}$ & $696(30.1)$ & $833(31.3)$ & NS \\
\hline ISH in $\mathrm{IHC}+$ & 576 & 541 & \\
\hline A & $69(12)$ & $87(16.1)$ & 0.048 \\
\hline NA & $471(81.8)$ & $348(64.3)$ & $\mathrm{s}$ \\
\hline E & $22(3.8)$ & $82(15.2)$ & $\mathrm{s}$ \\
\hline $\mathrm{U}$ & $14(2.4)$ & $8(1.5)$ & NS \\
\hline ISH in IHC $0 / 1+$ & $103(4.5)$ & $275(10.3)$ & $\mathrm{s}$ \\
\hline A & $3(2.9)$ & $2(0.7)$ & \\
\hline NA & 97 (94.2) & $249(90.5)$ & NS \\
\hline E & 0 & $20(7.3)$ & \\
\hline $\mathrm{U}$ & $3(2.9)$ & $4(1.5)$ & \\
\hline
\end{tabular}

$\mathrm{A}=$ amplified; $\mathrm{NA}=$ not amplified; $\mathrm{E}=$ equivocal; $\mathrm{U}=$ =uninterpretable; $\mathrm{S}=$ significant $\mathrm{p}<0.002 ; \mathrm{NS}=$ not significant

* 17 IHC $3+$ cases were tested by ISH in each group and all of these were amplified. Conclusions: Implementation of the 2013 updated guideline recommendations results in increase in overall tests due to primary testing CNB and repeat testing as required, however, there is no change in the \% of HER2+ve. There is a significant increase in cases categorized as equivocal by ISH in both IHC $2+$ and IHC $0 / 1$. The benefit of targeted therapy in this group is not proven and the rationale for having this category needs to be considered in future iteration on the guideline.

\section{Impact of the Change of the Criteria for HER2 Immunohistochemistry} Interpretation on HER2 Status in Breast Cancer

Shuko Harada, Dejun Shen, Shi Wei. University of Alabama at Birmingham, Birmingham, AL.

Background: It has been more than one year since ASCO/CAP updated the guidelines for HER2 immunohistochemistry (IHC) interpretation for invasive breast cancer (IBC). The major change was that IHC overexpression $(3+)$ is defined as complete membranous staining in $>10 \%$ of the cells in the 2013 guidelines as compared to $>30 \%$ of the cells in the 2007 guidelines. The aim of this study was to evaluate if the guideline changes had an impact on the HER2 IHC interpretation and on the concordance between IHC and ISH (in-situ hybridization) results.

Design: All cases of IBC evaluated for HER2 status between April 2014 and March 2015 at UAB were reviewed retrospectively. HER2 IHC was performed using anti c-erb2 antibody clone SP3 and ISH was performed using INFORM HER2 DualISH (Ventana) on sections of breast biopsy or resection specimens. HER2 IHC results were evaluated using both 2007 and 2013 criteria and the results were compared with ISH results. Since DualISH criteria did not have equivocal before 2013 guideline, the same criterial was applied to evaluate ISH status (i.e. no equivocal).

Results: A total of 844 IBC cases were evaluated for HER2 status, of which 789 had both IHC and ISH results. HER2 IHC evaluation using the 2013 criteria resulted in an increased number of overexpression ( $3+$ ) from 79 to 87 , and a decreased number of equivocal cases $(2+)$ from 63 to 55 . Criteria changes had no impact on the cases of IHC negative $(0 / 1+)$. Among the 8 cases $(1.0 \%)$ that were changed from $2+$ to $3+, 6$ were amplified by ISH whereas 2 remained non-amplified. The changes could potentially decrease the number of reflex ISH cases, however, two cases with IHC 3+ ISH could have been classified incorrectly if ISH were performed only on $2+$ cases. Overall, the discordance rates were low using both the 2013 (2.0\%) and the 2007 (1.8\%) criteria.

\begin{tabular}{|c|c|c|c|c|c|c|}
\hline & \multicolumn{5}{|l|}{ IHC } \\
\hline & & $\begin{array}{l}\text { Neg } \\
(0 / 1+)\end{array}$ & $\begin{array}{l}\text { Equivocal } \\
(2+)\end{array}$ & $\begin{array}{l}2+\text { in } 2007 \text { and 3+ } \\
\text { in } 2013\end{array}$ & $\begin{array}{l}\text { Overexpressed } \\
(3+)\end{array}$ & Total \\
\hline \multirow{3}{*}{ ISH } & $\begin{array}{l}\text { Non- } \\
\text { Amp }\end{array}$ & $\begin{array}{l}635 \\
(80.5 \%)\end{array}$ & $39(4.9 \%)$ & $2(0.3 \%)$ & $1(0.1 \%)$ & $\begin{array}{l}677 \\
(85.8 \%)\end{array}$ \\
\hline & AMP & $\begin{array}{l}12 \\
(1.5 \%)\end{array}$ & $16(2.1 \%)$ & $6(0.7 \%)$ & $78(9.9 \%)$ & $\begin{array}{l}112 \\
(14.2 \%)\end{array}$ \\
\hline & Total & $\begin{array}{l}647 \\
(82.0 \%)\end{array}$ & $55(7.0 \%)$ & $8(1.0 \%)$ & $79(10.0 \%)$ & 789 \\
\hline
\end{tabular}

Conclusions: Our study indicated that overall impact of guideline changes on IHC interpretation was minimal. The new guidelines slightly increased the sensitivity $(86.7 \%$ to $87.5 \%)$ and decreased the specificity ( $99.8 \%$ to $99.5 \%)$ if using ISH results as gold standard. In rare occasion, the 2013 criteria might lead to a false positive result by IHC when HER2 is not amplified by ISH. 
169 Correlation of p16 Expression on Cancer and Stromal Cells with Clinicopathological and Immunohistochemical Features of Lobular Breast Carcinoma

Aparna Harbhajanka, Ihab Lamzabi, Richa Jain, Ritu Ghai, Vijaya B Reddy, Paolo Gattuso. Rush University Medical College, Chicago, IL.

Background: Cancer associated fibroblasts (CAF), play a central role in the complex process of tumour-stroma interaction and, consequently, growing evidence suggests that they promote tumorigenesis, neoplastic progression, angiogenesis and metastasis. However, it is still unclear how these processes are regulated. The aim of this study is to investigate whether the p16 expression in cancer and stromal cells affects tumor biology and behavior of invasive lobular carcinoma(ILC).

Design: Clinicopathologic parameters and Immunohistochemical (IHC) stains for ER, PR, E-cadherin, HER2, and MIB-1 of 70 ILC cases were retrieved. In addition, IHC were performed for $\mathrm{p} 53$, p16, and cyclinD1 on 70cases. The patient with $\mathrm{p} 16$ expression in cancer cells and stromal cells were divided in 4 groups as follows; p16(-)cancer and stromal cells, p16(-)cancer cells and p16(+)stromal cells, p16(+)cancer cells and p16(-) stromal cells and p16(+)cancer and stromal cells and were correlated with respect to different clinicopathologic parameters.

Results: Of the 70 cases, 8 cases were p16(-)cancer and stromal cells, 14 p16(-)cancer and p16(+) stromal cells, 14 p16(+)cancer and p16(-)stromal cells, and 34 p16(+)cancer and stromal cells. $31 / 59$ cases showed axillary lymph node metastases. Lymph node metastasis was absent in 28 cases, micrometastasis was present in 3 cases, and stage $\mathrm{N} 1, \mathrm{~N} 2, \mathrm{~N} 3$ in 15, 1, and 12 cases respectively. Nodal involvement, recurrence and metastasis of ILC with p16(+)cancer cells and p16(-) stromal cells were more frequent compared to other groups (p-value-0.025 and 0.02). ILC with p16(+)cancer cells and p16 (-) stromal cells were frequently negative for ER, PR and cyclin D1, p53 positive and triple negative compared to other groups. There was no recurrence and metastasis in ILC with p16(-) cancer cells and p16(+) stromal cells. ILC with p16 (+)cancer and stromal cells were significantly node negative and positive for ER and cyclin D1 compared to other groups. ( $\mathrm{p}=0.039,0.018$ respectively).

Conclusions: Invasive lobular carcinoma with p16 (+)cancer cells and p16(-)stromal cells were characterized by frequent nodal involvement, recurrence and metastatic propensity. These results suggest that $\mathrm{p} 16$, in addition to its well-known anti-tumor proliferation function, has novel anti-cancer properties capable of suppressing cancer cell migration and invasion and pharmacologic restoration of $\mathrm{p} 16$ level in stromal fibroblasts may be exploited as therapeutic strategy to prevent their nodal or distant metastasis.

170 Mitotic Index after Neoadjuvant Chemotherapy Prognosticates Breast Cancer Recurrence or Metastasis

Nicholas Harding-Jackson, Christopher Hartley, Zainab Basir. Medical College of Wisconsin, Milwaukee, WI.

Background: Neoadjuvant chemotherapy for invasive breast cancer is established treatment for locally advanced disease. Several studies have examined the pre- and post-chemotherapy features to determine predictive and prognostic markers in evaluating the need for subsequent chemotherapy regimens. Higher pre-therapy proliferation is understood to show a better response to therapy, and proliferative indices are expected to fall following therapy. Previous studies have demonstrated that post-therapy Ki-67 is an independent prognostic variable in breast cancer (PMID 18592370). However, to our knowledge, the prognostic significance of post-neoadjuvant mitotic index has not been evaluated.

Design: 205 cases of residual invasive mammary carcinoma previously treated with neoadjuvant chemotherapy from 2007 through 2014 were reviewed retrospectively. Tumors classified as AJCC stage ypT $1 \mathrm{~b}(>5 \mathrm{~mm})$ or greater with $>15 \%$ cellularity were re-graded using the modified Bloom-Richardson (BR) grading system, as well as a raw mitotic count per 10 high power fields (40x). Using patient medical records, the following information was also obtained: primary diagnosis, age at diagnosis, ER/PR/ HER2 status, vital status, and presence of metastasis or local recurrence.

Results: 50 cases of residual carcinoma of ypT $1 \mathrm{~b}$ or greater with $>15 \%$ tumor cellularity were identified. 17/50 (34\%) had documented radiologic or histologic evidence of recurrence or metastasis. The median mitotic count for cases with documented recurrence or metastasis was $4 / 10 \mathrm{HPF}$, compared to $1 / 10 \mathrm{HPF}$ in cases with no evidence of recurrence or metastasis, $\mathrm{P}=0.023$. Cases showing Mitotic indices corresponding to BR mitotic grades 1-2 had an incidence of recurrence or metastasis of $9.1 \%$, compared to $35.8 \%$ for BR mitotic grade 3, after mean follow-up of 4.6 years, $\mathrm{P}=0.041$

\begin{tabular}{|l|l|l|l|}
\hline Outcomes vs mitotic count and Bloom-Richardson mitotic grade (BR) \\
\hline Outcome & $\begin{array}{l}\text { Mitotic index } \\
\text { Median (range) }\end{array}$ & $\begin{array}{l}\text { BR 1-2 } \\
(0-14 / \mathrm{HPF})\end{array}$ & $\begin{array}{l}\text { BR 3 } \\
(>14 / \mathrm{HPF})\end{array}$ \\
\hline No disease, $\mathrm{n}=33$ & $1(0-36)$ & $30(90 \%)$ & $3(9 \%)$ \\
\hline Recurrence or Metastasis, $\mathrm{n}=17$ & $4(1-82)$ & $11(65 \%)$ & $6(35 \%)$ \\
\hline Death (cumulative incidence), $\mathrm{n}=8$ & $6(1-82)$ & $5(63 \%)$ & $3(38 \%)$ \\
\hline
\end{tabular}

Conclusions: Post-neoadjuvant mitotic index is an independent prognostic variable in breast cancer. A morphologic assessment of mitotic index may obviate the use of Ki-67 in predicting disease recurrence or metastasis, and may be of utility in guiding more aggressive adjuvant therapy regimens. Compared to BR mitotic grades 1 and 2, BR grade 3 is $3.8 \mathrm{X}$ more likely to show local recurrence or metastasis.
171 Comparison of Immune Response and PDL1 Distribution in Triple Negative Invasive Breast Carcinomas in Different Ethnic Populations

Jian He, Arundhati Rao, Arun Sreekumar. Baylor, Scott \& White Memorial Hospital, Temple, TX; Baylor College of Medicine, Houston, TX.

Background: Triple negative breast cancers (TNBC) are among the most immunogenic of hormonally defined subsets and tumor-infiltrating lymphocytes (TILs) are associated with better prognosis in triple negative breast cancers. Recently increased expression of programmed cell death receptor ligand 1 (PDL1) expression in this group has also been reported. Very limited data is available on TILs of TNBC in the different ethnic populations and if $\mathrm{PD}-\mathrm{L} 1$ can be used as a prognosis or predictive therapeutic marker. Our study aims to investigate if the expression of PD-L1 is related to TILs in TNBCs and their clinical relevance in TNBCs in self-reported Caucasian (CA) and African American (AA) groups.

Design: A search of the Baylor, Scott \& White Hospital database identified 47 AA women and $52 \mathrm{CA}$ women with triple negative breast cancer resection samples with a minimum of 5 year follow up and treatment data. Immunohistochemistry for PD-L1, $\mathrm{CD} 3, \mathrm{CD} 4, \mathrm{CD} 8$ and $\mathrm{CD} 68$ were performed on constructed tissue microarray slides using areas of highest inflammatory infiltrate and analyzed with assisted image analysis (Applied Spectral Imaging/ASI).

Results: The mean CD3 count in the AA group is 1144 (Median=905, SD $=174,95 \%$ $\mathrm{CI}=350$ ) while the mean $\mathrm{CD} 3$ count of $\mathrm{CA}$ group is 800 (Median $=439, \mathrm{SD}=133,95 \%$ $\mathrm{CI}=267)(\mathrm{p}=0.06)$. Expression of PD-L1 in tumor cells was observed in $60 \%$ of AA TNBCs (27/45) and $38 \%$ of CA TNBCs (19/50). (The Chi-square statistic $\mathrm{p}=0.03)$. If $\mathrm{CD} 3$ count was less than median of AA group, the frequency of expression of PD-L1 is $27 \%(6 / 22)$ vs $91 \%(21 / 23)$. If CD3 count was less than median of CA group, the frequency of expression PD-L1 is $12 \%$ (3/25) vs $64 \%$ (21/25).

The mean CD4 count in the AA group is 253 (Median=103, $\mathrm{SD}=409,95 \% \mathrm{CI}=124$ ) and the CA group is 428 (Median $=179, \mathrm{SD}=540,95 \% \mathrm{CI}=153)(\mathrm{p}=0.18)$. The mean $\mathrm{CD} 8$ count in the AA group is 279 (Median $=185, \mathrm{SD}=605,95 \% \mathrm{CI}=184$ ) and in the $\mathrm{CA}$ group is 343 (Median $=123, \mathrm{SD}=367,95 \% \mathrm{CI}=103)(\mathrm{p}=0.07)$. The mean $\mathrm{CD} 68$ count in the AA group is 301 (Median=179, $\mathrm{SD}=331,95 \% \mathrm{CI}=100$ ) and the CA group is 92 $($ Median $=56, \mathrm{SD}=101,95 \% \mathrm{CI}=28)(\mathrm{p}<0.01)$.

Conclusions: Our data suggest that TILs in the AA TNBCs group is higher than in the CA cohort and correlates with a higher PD-L1 expression. This data could have therapeutic implications but may require a larger study for true assessment of significance.

\section{Prediction of Oncotype DX Recurrence Score: Use of a Pathology-} Generated Equation

Yanjun Hou, Gary Tozbikian, Debra L Zynger, Zaibo Li. The Ohio State University Wexner Medical Center, Columbus, $\mathrm{OH}$.

Background: The 21-gene Oncotype DX assay (Genomic Health, CA) is a RT-PCR based assay to estimate distance recurrence and guide chemotherapy decision for patients with estrogen receptor (ER) positive and node negative breast cancers. Recently, estimated recurrence scores (ERS) from Magee equations derived by linear regression analysis (based on tumor size, grade, hormone receptors and HER2 status) demonstrated relatively high concordance with the recurrence score of Oncotype DX assay.

Design: 313 cases with Oncotype DX recurrence score were analyzed by a modified Magee equation (equation \# 2) to predict recurrence score.

Results: The Pearson correlation coefficient for the Oncotype DX and modified ERS was $0.6068(\mathrm{p}<0.001)$. There was an overall $63.6 \%(n=199)$ agreement between the modified ERS and the Oncotype DX recurrence score. 98.2\% ( $\mathrm{n}=171)$ of cases with modified ERS $<18$ had an Oncotype DX recurrence score $<30$ (range 0 - 29). $73.1 \%$ $(125 / 171)$ of these cases had an Oncotype DX recurrence score $<18$ (likelihood ratio $=2.32, \mathrm{p}<0.001) .87 .5 \%(7 / 8)$ of cases with modified ERS $>30$ had Oncotype DX recurrence score $>30$

Table 1. Correlation between Magee equation scores and Oncotype Dx scores

\begin{tabular}{|c|l|l|l|l|}
\hline \multirow{2}{*}{ ERS (Modified Magee equation \# 2) } & \multicolumn{4}{|c|}{ Oncotype DX recurrence score } \\
\cline { 2 - 5 } & $<18$ & $18-30$ & $>30$ & Total \\
\hline$<18$ & 125 & 43 & 3 & 171 \\
\hline $18-30$ & 44 & 67 & 23 & 134 \\
\hline$>30$ & 0 & 1 & 7 & 8 \\
\hline Total & 169 & 111 & 33 & 313 \\
\hline
\end{tabular}

Conclusions: Our findings support that the use of modified Magee equation combined with standard H\&E and immunohistochemistry, may constitute a cost-effective alternative to Oncotype DX.

173 Strong ROR1 Expression Is an Independent Adverse Prognostic Factor in Triple Negative Invasive Breast Carcinoma

Chuen Hsueh, Hui-Ping Chien, Shin-Cheh Chen, Yu-Sun Chang, Shir-Hwa Ueng, YungChang Lin, Yun-Feng Lo, Hsien-Kun Chang, Wen-Yu Chuang. Chang Gung Memorial Hospital, Linkou, Taoyuan, Taiwan; Chang Gung Memorial Hospital, Keelung, Keelung, Taiwan; Chang Gung University College of Medicine, Taoyuan, Taiwan.

Background: Type 1 receptor tyrosine kinase (RTK)-like orphan receptor 1 (ROR1) is an oncofetal protein of importance in normal embryonal development. Overexpression of ROR1 in several human malignancies is associated with aggressive behavior. In breast cancer, high ROR1 expression is associated with higher grade cancers, distant metastasis and shorter overall survival (OS). Therapeutic agents targeting ROR1 have shown promising results in vivo and in vitro studies. Triple negative invasive breast carcinoma (TNBC) is known to have a worse prognosis with no available target therapy. The study was aimed to assess the potential prognostic and therapeutic usefulness of ROR1 expression in TNBC 
Design: We retrieved 210 TNBC mastectomy specimens from our departmental file between 2005 and 2010. Immunostaining of ROR1 was evaluated by two pathologists and only cytoplasmic staining was considered positive. The result was correlated with age, tumor size, tumor grade, lymph node status, TNM stage, and patient survival. The expression of ROR 1 was assessed by extent $(1=1-<30 \%$ positive tumor cells, $2=30-75 \%$, $3=>75 \%)$ and intensity $(1=$ weak, $2=$ moderate or $3=$ strong $)$ of the staining. Two scores were multiplied to give a total score of 0 to 9 .

Results: Strong ROR1 expression (intensity score 3) was present in 47 cases $(22.4 \%)$ and correlated with tumor recurrence $(\mathrm{P}=0.003)$. It was associated with shorter diseasefree survival (DFS), distant metastasis-free survival (DMFS) and OS by both univariate $(\mathrm{P}<0.001,<0.001,=0026$, respectively $)$ and multivariate analyses $(\mathrm{P}<0.001,<0.001$, $=0.031$, respectively), while total score had no statistical significance. In addition, 70 TNBC cases $(33.3 \%)$ showing medullary features which was associated with longer DFS, DMFS and OS $(\mathrm{P}=0.012,=0.011,<0.001$, respectively). When two groups were divided based on the presence or absence of medullary features, strong ROR 1 expression remained significant for DFS and DMFS in both former $(\mathrm{P}=0.018,=0.006)$, and latter $(\mathrm{P}=0.004,=0.0043)$ groups.

Conclusions: Our study found that strong ROR 1 expression is an independent adverse prognostic factor in TNBC patients, thus it may serve as a potential marker for patient selection for ROR1-targeted therapy.

\section{Breast Pathologic Diagnosis and BIRADS Classes Correlation} Study on $\mathbf{4 1 1 8}$ Patients

Shaomin Hu, James Szymanski, Yungtai Lo, Yanhua Wang. Montefiore Medical Center/ Albert Einstein College of Medicine, Bronx, NY; Albert Einstein College of Medicine, Bronx, NY.

Background: Standardized Breast imaging-reporting and data system ${ }^{\circledR}$ (BIRADS) has been used to classify breast imaging findings. Although recent technological advances, there is still discordance between breast imaging findings and pathologic diagnosis. This study aims to correlate the breast imaging BIRADS classes with pathologic diagnosis. Design: Female patients who had a breast imaging test and a reported BIRADS class from $01 / 01 / 2010$ to $05 / 16 / 2014$, and had a breast biopsy/excision within 90 days of the breast imaging test, were identified using $C L_{G}{ }^{\circledR}$ software. Each patient's pathologic diagnosis (the first one if more than one) was assigned to a category either 'malignant' or 'benign'. Patients were then divided into 7 groups based on their BIRADS classes $(0$, $1,2,3,4,5$ and 6$)$. For each group, the percentage of benign and malignant pathologic diagnosis was analyzed, the patients' racial, socioeconomic status (SES), age and BMI distributions between benign and malignant group were compared.

Results: A total of 99,071 patients with a reported BIRADS class were analyzed. 4,118 patients had a pathology report within 90 days, with an average age of 53 years. 1,564 (38.0\%) were African Americans, 1,321 (32.1\%) were Hispanics/Latinos, 450 (10.9\%) were Whites and $783(19.0 \%)$ were other races. $260(6.3 \%)$ of 4,118 patients were reported as BIRADS 0, $342(8.3 \%)$ were BIRADS 1, $332(8.1 \%)$ were BIRADS 2, 271 (6.6\%) were BIRADS 3, 2,551 (61.9\%) were BIRADS 4, 231 (5.6\%) were BIRADS 5, and $131(3.2 \%)$ were BIRADS 6. Malignant pathologic diagnosis was found in $35.4 \%$ of patients with BIRADS $0,15.8 \%$ of patients with BIRADS $1,13.9 \%$ of patients with BIRADS 2 , and $7.7 \%$ of patients with BIRADS 3. Under the same BIRADS class, patients with malignant diagnosis were older $(\mathrm{p}<0.01)$ and more likely to be African American or White compared to those with benign pathologic findings. SES and BMI were not significantly different between the two groups.

Conclusions: Significant percentage (up to $15.8 \%$ ) of patients with BIRADS 1-3 had malignant breast pathologic findings in this study. African American or white patients and those with older age are at higher risk. Further studies will address the underlying reasons for the patients with BIRADS 1-3 to receive breast biopsy/excision (SH and JS contributed equally to this study).

\section{HER2 FISH Testing with RAl1 Alternate Probe Will Resolve HER2} Status in Majority of Equivocal Cases

Ling Hui, Katherine Geiersbach, Erinn Downs-Kelly, Rachel E Factor, H Evin Gulbahce. University of Utah, SLC, UT; ARUP, SLC, UT.

Background: The 2013 ASCO/CAP guidelines changed the equivocal (EQ) category for HER 2 in situ hybridization (ISH) testing. A HER 2 copy number $\geq 4-5.9$ per cell with a HER2:CEP17 ratio $<2$ is EQ and alternate testing methods are recommended. These guidelines propose an alternative probe to avoid false negative results due to increased $C E P 17$ copy number. Alternate loci on chromosome 17, which are not expected to coamplify with HER2, have been used for this purpose. RAII (previously SMS) makes an attractive alternative locus as TCGA data showed $<1 \%$ high level $R A I 1$ amplification (Amp) in breast cancers (BC) with $<0.1 \%$ of HER2 Amp BC also co-amplified for $R A I 1$. However, up to $37 \%$ of $\mathrm{BC}$ may show a loss of $R A I 1$, potentially resulting in over estimation of $H E R 2$ Amp cases. We review our experience with the RAI1 probe following EQ HER2 FISH.

Design: HER2 FISH testing performed between April 2015, when the alternate RAI1 probe was available in our lab, and July 2015 were identified. Information on site (breast versus gastric), prior Hercept IHC testing (if done), and FISH results with an FDA approved (CEP17) and alternate (RAI1) probe were retrieved from electronic files including HER2, CEP17, RAII copy numbers per cell as well as ratios. Deletion of RAII was defined as $R A I 1$ copy number per cell $<1.5$ and 17 or more out of 40 cells with only 1 RAII signal per cell. H+E stained slides were reviewed to determine histologic type, morphologic features and grade.

Results: HER2 FISH testing performed with CEP17 as the reference probe, identified 52 breast primary cases which were EQ. Testing with the $R A I 1$ probe identified $34.6 \%$ (18/52) as Amp with a HER2:RAI1 ratio ranging from 2.0 to 2.9 (average ratio: 2.4); $61.5 \%(32 / 52)$ were non-amplified (NonAmp), and 3.8\% (2/52) were still unclassifiable owing to deletion of RAII. Tumors that had EQ HER2 FISH requiring alternate RAII testing had the following morphologic features: 46 ductal NOS, 2 ductal with lobular features, 3 lobular, and 1 cell block is not-classifiable. Histomorphology of the 18 cases that were Amp following RAI1 testing were as follows: ductal $(\mathrm{n}=16)$, ductal with lobular features $(n=1)$, lobular $(n=1)$.

Conclusions: The RAI1 alternate probe following EQ HER2 FISH will resolves HER2 status in the majority $(96.2 \%)$ of cases. Close to $35 \%$ of cases were designated as Amp following testing with $R A I 1$, making them eligible for targeted therapy. In our series, deletion of RAII was much lower than reported in the previous literature with only $3.8 \%$ of cases not resolved due to deletion of RAII1.

\section{CD3+ and FOXP3+Tumor-Infiltrating Lymphocytes Are Associated} with Better Prognosis in Triple Negative Breast Cancers

Jabed Iqbal, Joe Yeong, Jeffrey Chun Tatt Lim, Puay Hoon Tan. Singapore General Hospital, Singapore, Singapore.

Background: Triple negative breast cancers (TNBCs) are defined by the absence of estrogen receptor (ER), progesterone receptor (PR) and c-erbB2 expression. Recently tumor-infiltrating lymphocytes (TILs) have been reported to associate with survival and therapy response in a variety of cancers including breast cancer. FoxP3 is a marker for immunosuppressive CD4+CD25+ regulatory T cells (Tregs). In this study, we investigate the role of CD3+ and Foxp3+ TILs as candidate biomarkers for clinical outcome in a cohort of TNBCs in Singapore.

Design: The cohort comprised 164 TNBCs diagnosed from 1994 to 2010. The antibodies of CD3 and Foxp3 antibodies were applied to sections obtained from tissue microarray blocks. Following recommendations by the International TILs Working Group, TILs were counted as stromal when within cancer stroma (sTILs). TILs with direct contact with carcinoma cells within cancer cell nest were considered as intratumoral (iTILs). A median cut-off value was used to define low and high TILs groups. Disease free survival (DFS) and overall survival (OS) were correlated with CD3+ and Foxp3+ TILs. A p-value $<0.05$ defined statistical significance.

Results: By using a median cut-off, the presence of high CD3+ iTILs and sTILs were observed in $52.8 \%$ and $52.2 \%$ of the cases respectively while the presence of high Foxp3+ TILs, both intratumoral and stromal, were observed in $42.1 \%$ and $56.6 \%$ of the cases respectively. Foxp $3+$ stromal TILs showed significant association with higher tumor grade $(\mathrm{p}=0.025)$ and lymph node status $(\mathrm{p}=0.039)$. The iTIL Foxp3/CD3 ratio was inversely associated with tumor size $(0.021)$.

According to Kaplan Meier analysis, high CD3 in both intratumoral and stromal compartments were associated with better DFS [iTILS $(p=0.019)$ and sTILS $(p=0.019)$ ] and OS [iTILS $(\mathrm{p}=0.0050)$ and sTILS $(\mathrm{p}=0.018)$ ]. Low Foxp3+iTILs but not sTILs were associated with worse DFS $(\mathrm{p}=0.001)$ and the result was confirmed by multivariate survival analysis (HR 3.926, 95\% CI 1.477-10.441, $\mathrm{p}=0.006$ ).

Conclusions: High CD3+ iTILs and sTILs as well as Foxp3+ iTILs augur a better survival oucome in TNBCs patients. Foxp3+ iTILs was shown to be an independent prognostic factor in this study. However, a high Foxp3 sTILs correlated with higher tumor grade and lymph node metastasis. Foxp3 $+\mathrm{T}$ regulatory cells possibly have a different role in the two copmartments within TNBC tumor microenvironment. The interaction between tumor and TILs, both intratumoral and stromal, should be further interrogated to facilitate the use of TILs as biomarkers.

\section{Increased BCL2 Expression Predicts Poor Survival in Triple Negative Breast Cancer Patients}

Jabed Iqbal, Aye Aye Thike, Takashi Yamasaki, Joe Yeong, Puay Hoon Tan. Singapore General Hospital, Singapore, Singapore; Kobe University School of Medicine, Kobe, Japan.

Background: Triple negative breast cancer (TNBC) represents a subgroup of breast cancers that lack expression of estrogen receptor (ER), progesterone receptor (PR) and human epidermal growth factor-2 (HER2). TNBCs are generally aggressive tumours and represent a heterogeneous group of tumours with regards to clinical presentation, pathology, prognosis and response to treatment. Recent studies have shown the antiapoptotic $\mathrm{Bcl} 2$ protein and gene expression to be a promising prognostic and predictive marker in ER-positive, node-negative breast cancers whereby studies have reported mostly favorable prognosis in ER-positive breast cancer pateints receiving adjuvant endocrine therapy. The role of $\mathrm{Bcl} 2$ in TNBC, however, is not so well-defined.

Design: The cohort comprised 164 TNBCs diagnosed from 1994 to 2010 . BCL2 antibody was applied to sections obtained from tissue microarray blocks. The tumour cores were evaluated by two pathologists for concordance. Positive $\mathrm{Bcl} 2$ cytoplasmic expression was defined as $\mathrm{H}$ score of $>100$. Disease free survival (DFS) and overall survival (OS) were correlated with BCL2 expression. A p-value $<0.05$ defined statistical significance.

Results: Mean age of TNBC patients in our study was 54y (range 24y-84y). Bcl-2 expression was not associated with any of the clinicopathologic parameters. Only $38 \%$ of Bcl2-positive tumors showed LVI. Majority of TNBC cases were $\mathrm{Bcl} 2$ negative and positive $\mathrm{Bcl} 2$ expression was observed in $14 \%$ patients. $\mathrm{Bcl} 2$ expression was significantly associated with basal-like phenotype and was inversely associated with high tumor grade. On univariate analyses, Bcl2-positivity was associated with poor DFS $(0.041)$ and $O S(P=0.044)$ in TNBC patients.

Conclusions: High Bcl2 expression appears to associated with basal phenotype and is a predictor of poor outcome in TNBC; low or negative BCL2 expression was associated with better survival outcome. Studies on the role of BCL2 expression in TNBC outcome has yielded mixed results in the literature. Our sample size may be a limiting factor and further studies on a larger TNBC cohort is warranted. Standardizing BCL-2 expression analysis is also important to appreciate the role of BCL-2 in prognosis. 
178 The Role of a Negative Axillary Fine Needle Aspiration in Identifying Breast Cancer Patients with No Axillary Metastasis

Mark Jabbour, Mothana Saadeldine, Ghina Berjawi, Faek Jamali, Jaber Abbas, Fouad Boulos. American University of Beirut Medical Center, Beirut, Lebanon.

Background: Previous work (Jabbour et al USCAP 2014) showed that a positive Fine Needle Aspiration (FNA) failed to accurately predict the number of involved lymph nodes in breast cancer patients, with $43 \%$ of cases demonstrating $\leq 2$ involved lymph nodes. The aim of the current study is to determine whether a negative axillary FNA precisely identifies patients with a negative subsequent axillary dissection and/ or sentinel lymph node biopsy.

Design: Following review of the pathology and cytology database between 1996-2014, a total of 36 cases of negative FNA of the axilla with a corresponding axillary dissection and/or sentinel lymph node biopsy were examined. Clinicopathologic characteristics including age, tumor histology, size, lymph node involvement, metastasis size, extranodal extension and neoadjuvant therapy were recorded.

Results: The median age of the studied patients was 51 years (range: $37-79$ ). $83.3 \%$ $(30 / 36)$ cases were IDC, $5.6 \%(2 / 36)$ were ILC, $8.3 \%(3 / 36)$ were mixed and $2.8 \%$ $(1 / 36)$ was adenosquamous carcinoma. $52.8 \%(19 / 36)$ were grade $3,33.3 \%(12 / 36)$ were grade 2 and $8.3 \%(3 / 36)$ were grade 1 while in $5.6 \%(2 / 36)$ histologic grade was not available. Median tumor size was $1.8 \mathrm{~cm}$ (range: $0.1-5 \mathrm{~cm}$ ). Lymph node involvement was identified in $33.33 \%(12 / 36)$ of cases with a median size of $0.9 \mathrm{~cm}$. Extranodal extension was present in $33.33 \%$ (4/12) of involved lymph nodes. $8.3 \%$ (3/36) of cases received neoadjuvant chemotherapy with $1 / 3$ cases showing absent consequent lymph node involvement. The negative predictive value (NPV) of a negative FNA was $66.7 \%$ with a corresponding rate of positive lymph nodes equivalent to $33.3 \%$. Excluding micrometastasis $(n=3)$, the NPV reaches $72.7 \%$ while the rate of positive lymph nodes equals $27.3 \%$. There was no statistically significant correlation between any of the recorded clinicopathologic parameters and the axillary lymph node status following dissection/sentinel node sampling.

Conclusions: A negative axillary FNA fails to predict a negative axilla in breast cancer patients in a significant proportion of cases. Given the current importance of quantitative rather than qualitative assessment of axillary lymph node status prior to axillary clearance, and the lack of such predictive value in axillary lymph node aspiration, the role of FNA in axillary staging and management decisions is challenged, while the role of sentinel node biopsy is reinforced.

\section{Intraductal Papillomas of the Breast on Core Biopsy, Re-Visiting} 10 Years Experience with More Than $\mathbf{5 0 0}$ Cases

Shabnam Jaffer, Bailey A Reindl, Ira J Bleiweiss, Anupma Nayak. Mount Sinai Medical Center, New York, NY.

Background: The management of pure intraductal papilloma (IDP) without atypia/ malignancy diagnosed on core biopsy (cbx) remains controversial. Due to the variable upgrade rates to atypia/malignancy, there is uncertainty regarding excision (exc) versus observation of cbx diagnosed IDP. At our institution, all targeted cbx diagnosed IDP have been consistently excised since 2000 . We previously published our upgrade rate to malignancy of $8.7 \%$ and atypia of $7.7 \%$ from $2000-2004$. We reevaluated these cases and assessed our upgrade rate from 2005-2015 on a larger dataset.

Design: Using the pathology database we retrospectively identified $512 \mathrm{cbx}$ diagnosed IDP that underwent exc. All specimens were reviewed to confirm the cbx and exc diagnosis, presence of cbx site on exc and performed radiologic-pathologic correlation. Results: The method of cbx was as follows: US for mass $=431(84.3 \%)$, stereotactic for calcifications $=63(12.3 \%)$ and MRI for enhancement $=18(3.5 \%)$. The diagnoses on exc were as follows: no residual IDP $=51(9.9 \%)$, residual $\mathrm{IDP}=407(79.5 \%)$, atypia $=30(5.9 \%)$, and ductal carcinoma in situ (DCIS) $=24(4.7 \%)$. The method of $\mathrm{cbx}$ in the DCIS cases were as follows: US $=19$, stereotactic $=2$ (granular calcifications) and MRI=3(linear enhancement=1). The US features in the DCIS cases were as follows: irregular mass $=4$, multilobulated mass from 0.5 to $3.0 \mathrm{~cm}=4$, cystic $/$ solid $=11$. $\mathrm{Cbx}$ diagnosis of IDP was confirmed in all cases. In 4 of the 24 cases of DCIS, pagetoid spread of atypical cells was seen involving IDP. In the DCIS exc, residual IDP was present in all cases but 2, involved by DCIS in 12 and atypia in 7 . The size of the DCIS ranged from $0.2 \mathrm{~cm}-0.7 \mathrm{~cm}$ and was either focal or widespread. In 2 cases the DCIS was at the cbx site, the remaining 22 away from it. The method of cbx for the ADH cases was $U S=23$, stereotactic $=5$ and $\mathrm{MRI}=2$. Residual IDP was present in all cases, involved by atypia in 8 , present at the cbx in 6 , and away in 24 . ADH was focal in 23 and scattered in 7.

Conclusions: The upgrade rate to atypia/malignancy improved over time. If we exclude the 4 cases of atypia involving IDP on cbx, the true upgrade rate to malignancy is $3.9 \%$. Of note unlike our previous experience, no invasive carcinomas were found. Given the high sampling error in the DCIS and atypia cases, we continue to recommend exc of IDP on cbx, especially since IDP are multilobulated, heterogeneous and irregular appearing on imaging. We noticed a trend towards higher use of US for detecting and sampling IDP with smaller needles, leaving a significant number of cases with residual IDP, which may potentially harbor atypia or malignancy.

\section{A Systematic Method of Assessing ER/PR/Her2 on Core Biopsy} and or Excision Specimens

Shabnam Jaffer, Bailey A Reindl, Ira J Bleiweiss, Anupma Nayak. The Mount Sinai Medical Center, New York, NY.

Background: It is standard practice to assess ER/PR and Her2 analysis on all invasive breast cancers. We have traditionally accomplished this by immunohistochemistry (ihc) on surgical excision (exc) specimens, preoperatively testing core biopsies (cbx) only in Stage IV patients and those being considered for neoadjuvant therapy. Recently there has been a demand from clinicians and patients to obtain this information preoperatively.
Thus in 2013 we changed our practice to reflexively test all invasive breast cancers diagnosed on cbx and repeat only initially negative tests on surgical exc. We evaluated the value of this practice.

Design: From 2013 to date, using the pathology database, we retrospectively identified 627 invasive carcinomas of the breast diagnosed on cbx that underwent ER/PR/Her2 testing by ihc. All Her2 equivocal cases were further tested by FISH. All specimens met the ASCO/CAP guidelines for fixation, and ihc stains for hormone receptor and Her2 were interpreted using ASCO/CAP guidelines.

Results: With the exception of 24 triple + cases $(\mathrm{ER}+/ \mathrm{PR}+/ \mathrm{Her} 2+)$, retesting was necessary in 1 or more of these components (ER/PR/Her2) in 603 cases on exc, as shown on the [table 1] below. Repeat testing on exc changed the status of 5 cases from being ER negative to $+($ ave $=6 \%$, range $1-20 \%$ ) and 20 cases from being PR negative to + (ave $=16 \%$, range $1-50 \%)$. Only 1 Her2 equivocal $(2+30 \%)$ case converted to + $(3+30 \%)$ on excision. FISH was attempted to resolve the equivocal result on the cbx but was technically inadequate. There were also 3 cases on cbx that converted from being Her2 equivocal to + by FISH testing on cbx.

\begin{tabular}{|l|l|l|l|l|l|l|}
\hline CBX & & & CBX & EXC & & \\
\hline ER & PR & HER2 & TOTAL & ER & PR & HER2 \\
\hline+ & + & + & 24 & 0 & 0 & 0 \\
\hline+ & + & - EQ & 470 & 0 & 0 & 470 \\
\hline+ & - & $-/ E Q$ & 54 & 0 & 54 & 54 \\
\hline- & - & $-/ E Q$ & 52 & 52 & 52 & 52 \\
\hline- & - & + & 14 & 14 & 14 & 0 \\
\hline+ & - & + & 8 & 0 & 8 & 0 \\
\hline- & + & - & 5 & 5 & 0 & 5 \\
\hline TOTAL & & & 627 & 71 & 128 & 581 \\
\hline
\end{tabular}

Conclusions: The repeat test rate $(780 / 627 \times 3(\mathrm{ER} / \mathrm{PR} / \mathrm{Her} 2=1881)$ on excision was approximately $41 \%$, mostly due to Her2 (74.4\%) retesting, followed by PR (16.4\%) and ER (9.1\%). Retesting exc specimens made a difference in $27(4.3 \%)$ patients, converting their status from hormone receptor negative to + (albeit low and weak in some cases), and Her 2 negative to + in only 1 patient. Repeating initially negative tests may result in treatment changes in a small number of patients; however, as we move toward a more cost conservative environment, we may need to reevaluate the efficacy of testing ER/PR/Her2 on cbx.

181 Minimal Clinical Impact of Intraoperative Examination of Sentinel Lymph Nodes in Patients with DCIS: An Opportunity for Improved Resource Utilization

Amy S Joehlin-Price, Debra L Zynger. The Ohio State University, Columbus, OH Background: Intraoperative sentinel lymph node (SLN) examination for patients with breast carcinoma is a widely utilized procedure that can guide operative management. However, the utility in the context of ductal carcinoma in situ (DCIS) has not been well elucidated. We examined the usage and clinical impact of intraoperative SLN frozen sections in patients with DCIS.

Design: Departmental records were searched for breast resection specimens with SLNs submitted from 2012-2013. Resection reports were analyzed for use of SLN frozen section, diagnostic results and pathology resource utilization (number of parts and blocks submitted). Diagnoses in prior biopsies were reviewed. Proportions of positive SLNs and pathology resource utilization variables were compared between DCIS and invasive carcinoma using chi-squared and Fisher exact tests. For reference, our institutional cryosection procedure includes serial sectioning SLNs to a thickness of 0.2 $\mathrm{cm}$ with three cryosections examined intraoperatively. Remaining tissue is submitted for permanent section, in which an additional two H\&E levels and one intervening cytokeratin immunostain are examined.

Results: 143 of 152 cases (94.1\%) with biopsy-proven DCIS had SLNs submitted for frozen section, significantly higher than the proportion of invasive cases with SLNs submitted for frozen section during the same time frame $(590 / 746,79.1 \% ; \mathrm{p}<0.001)$. Of the SLNs sent for frozen section with a history of DCIS, there were 10 SLN metastases with 3 macrometastases, 3 micrometastases and 4 isolated tumor cells. Only the macrometastases were identified at the time of cryosection and 2 of these resulted in synchronous axillary lymphadenectomies. Final pathology revealed significantly fewer DCIS cases with SLN positivity than those with a history of invasive carcinoma ( $6.6 \%$ in DCIS vs $27.4 \%$ in invasive carcinoma, $\mathrm{p}<0.001)$. No additional differences in resource use were identified between the in situ and invasive groups, to include no differences in SLN part types $(\mathrm{p}=0.097)$, total SLNs per case $(\mathrm{p}=0.374)$ or total SLN blocks per case $(\mathrm{p}=0.714)$.

Conclusions: Intraoperative SLN examination impacted patient management in only $1.4 \%$ of cases with a history of DCIS. As intraoperative SLN examination in the context of DCIS has minimal clinical impact, eliminating the routine use of this procedure represents an opportunity for health care cost containment. 
182 RNA-Seq of Pleomorphic \& Classic Lobular Carcinoma In Situ Identifies Significant Differences in Gene Expression Indicating Pleomorphic LCIS Resembles Luminal B-type Breast Cancer

Arthur Johnson, Christine Henzler, Molly Klein, Stuart Cameron, James Richter, Sumaya Al Rawi, Jessica Gruenberg, Aqsa Nasir, Maryam Shahi, Evan W Sell, Kathryn L Schwertfeger, Richard Zera, Andrew C Nelson. HCMC, Minneapolis, MN; University of MN, Minneapolis, MN.

Background: Classic lobular carcinoma in situ (cLCIS) is considered a marker for breast cancer risk rather than a precursor lesion. In contrast, pleomorphic LCIS (pLCIS) confers a higher risk of concomitant invasive lobular carcinoma (ILC), suggesting a direct precursor relationship between pLCIS and ILC. Previous work has shown pLCIS has lower ER expression, increased HER2 \& p53 expression, and higher Ki-67 labeling index compared to cLCIS. To further characterize the biologic differences between pLCIS \& cLCIS we completed RNA-seq analysis of these 2 entities.

Design: 198 cases with LCIS were identified from 2 separate institutions. 24 cases of cLCIS and 7 cases of potential pLCIS that were selected for independent review by 3 surgical pathologists. Only cases with consensus agreement were classified as pLCIS. RNA-seq was preformed using Illumina's TruSeq RNA Access Kit, with 2x100 bp reads and an average of over 27M reads/sample. Successful RNA-seq data was obtained from 19 cLCIS, 3 pLCIS, and 6 control samples consisting of normal breast tissue. Results: 1336 genes had significantly different expression levels in cLCIS or pLCIS in comparison to the controls. $44 \%$ were differentially expressed in both pLCIS and cLCIS, while $19 \%$ were unique to pLCIS and $38 \%$ were unique to cLCIS. Comparing pLCIS to cLCIS demonstrated 134 genes with significantly different expression between the two types of neoplasia. Pathway analysis indicated that the cLCIS vs pLCIS differential expression gene set is enriched for genes involved in receptor tyrosine kinase signaling (including ERBB2/HER2, RET, \& Retinoic Acid Receptor), inflammatory activation, cell proliferation, migration, and apoptosis. Furthermore, the set of genes differentially regulated in pLCIS support the conclusion that pLCIS is molecularly similar to Luminal B type breast cancer (based on 34 genes demonstrating coordinated regulation in comparison to published profiles of Luminal B breast cancer). Initial hierarchical clustering analysis distinguished 12 of the cLCIS cases into distinct clusters separate from the pLCIS cases, while 6 cLCIS cases showed some overlap with the pLCIS cases. Conclusions: This analysis suggests that the more aggressive behavior of pLCIS may be due to its biologic similarities to Luminal B type breast cancer. Validation of these findings should lead to molecular diagnostic methods that better characterize cLCIS vs pLCIS and identify potential mechanisms for therapeutic intervention.

183 Breast Frozen Section of Margins (FSM) Reduces Reexcision: The First 5 Years at an Ambulatory Surgical Center (ASC)

Julie Jorns, Michael Sabel, Stephanie Daignault, Angela Wu. University of Michigan, Ann Arbor, MI.

Background: In 2009 we established a specialized breast FSM practice at an ASC, leading to $36 \%$ and $34 \%$ reductions in reoperation (55.3\% to $19.3 \%)$ and margin reexcision ( $48.9 \%$ to $14.9 \%$ ) for breast conserving therapy (BCT) patients, respectively, in the first year. In light of evolving surgical practices in BCT we evaluated its impact in the first 5 years.

Design: All patients undergoing breast or axillary frozen section (FS) were included (2009-2014). FSM \& sentinel lymph node (SLN) FS patients had prior diagnoses of ductal or pleomorphic lobular carcinoma in situ (DCIS/pLCIS) and/or invasive carcinoma. SLN FS patients were clinically node-negative and had negative or deferred preoperative axillary staging. Breast FS, including FSM, was performed via a special technique using liquid nitrogen. Positive (tumor at ink) and close (tumor $<0.2 \mathrm{~cm}$; focal: $\leq 2$ foci with $<0.1 \mathrm{~cm}$ extent and extensive: $>2$ foci with $\geq 0.1 \mathrm{~cm}$ extent) margins, FS block volume, turnaround time (TAT) and clinicopathologic features were reviewed. Results: 1248 patients (mean age 57) undergoing 1303 procedures had: invasive ductal carcinoma $(65.9 \%)$, DCIS/pLCIS (18.6\%), invasive lobular carcinoma $(9.7 \%)$ and other (5.8\%) diagnoses. FS was performed for: lumpectomy (58.6\%), SLN (19.3\%), lumpectomy and SLN (15.4\%), reexcision (3.8\%), reexcision and SLN $(0.2 \%)$ and other $(2.9 \%)$ surgeries.

Requests for SLN FS declined (with a proportionate increase in FSM) over time. Of $1014 \mathrm{FSM}$ cases reexcision was performed in $39(3.9 \%), 48(4.7 \%)$ and $60(5.9 \%)$ with focally close, extensively close and positive margins, respectively, with overall reexcision rate of $14.5 \%$. Reexcision was lowest (12.3\%) in 2013-14, likely driven by a decrease in reexcision for focally close margins. Reexcision was significantly associated with $\geq \mathrm{pT} 2$ stage $(\mathrm{p}=.0005)$, lobular subtype $(\mathrm{p}<.0001)$ and multifocality $(\mathrm{p}<.0001)$. TAT was directly proportionate to block volume.

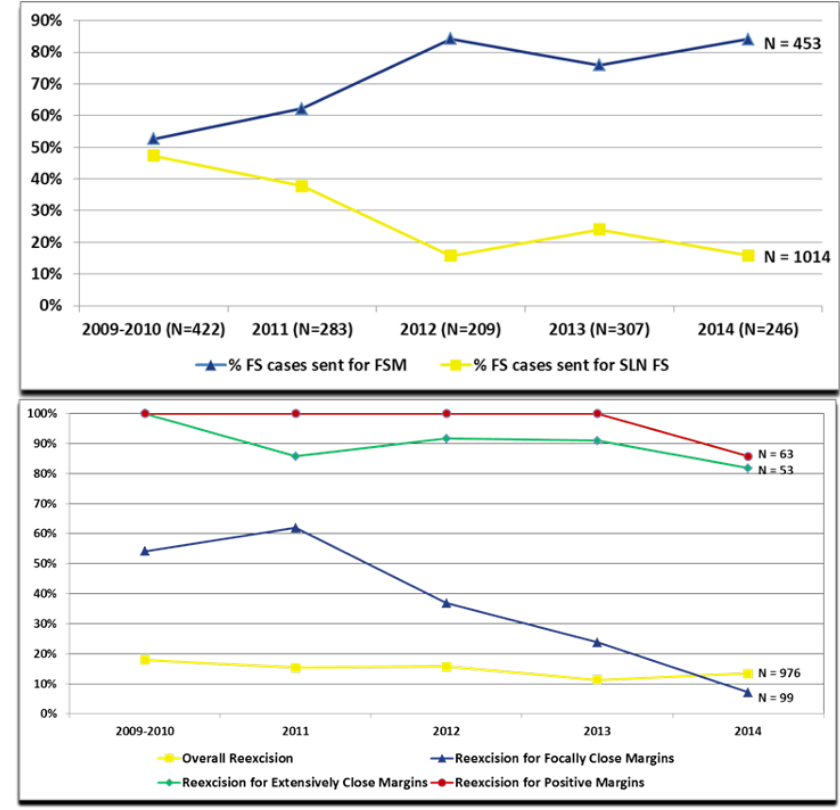

Conclusions: FSM decreases the liklihood of positive and/or close margins. Shifting surgical attitudes toward reexcision and continued use of FSM are likely to result in continued low reexcision rates.

184 Atypical Ductal Hyperplasia (ADH) Versus Ductal Carcinoma In-Situ (DCIS) in a Papilloma: Comparison of Linear Dimension and Proportion Criteria

Elizabeth Kalife, Kamaljeet Singh, C James Sung, M Ruhul Quddus. Women and Infants Hospital, Providence, RI.

Background: Linear dimension ( $\geq 3 \mathrm{~mm}$ ) and proportion ( $\geq 90 \%$ ) of atypia have been proposed as diagnostic cut-offs for DCIS in a papilloma. Recently, the size criterion of $\geq 3 \mathrm{~mm}$ was favored by the $\mathrm{WHO}$ consensus group, albeit as a pragmatic approach lacking firm evidence. The aim of our study is to compare the difference in categorization of ADH \& DCIS in a papilloma using these criteria.

Design: Core needle biopsies (CNBs)/excisions from 1/2007-7/2015 diagnosed as papilloma with ADH/DCIS were identified. Cases with intermediate-high nuclear grade DCIS, overt DCIS outside the papilloma or invasive carcinoma were excluded. Each case was reviewed by a breast pathologist to assess for the presence, linear extent and proportion of atypical ductal proliferation within the papilloma. Concomitant UDH, contiguity of the ADH/DCIS, number of ducts involved and extra-lesional ADH were also noted. Follow-up was included where available. Statistical analysis was performed using SPSS.

Results: In total, $41 \mathrm{CNBs}$ and 13 excisions from 54 patients qualified for the study. The mean size of the papillary lesion was $4.9 \pm 3 \mathrm{~mm}$ (range 1-14mm), mean linear extent of atypia was $2.99 \pm 2.39 \mathrm{~mm}(0.5-10 \mathrm{~mm})$ and mean proportion of atypia was $67.5 \pm 28 \%$ (10-100\%). The papillary lesion involved 1 duct in $19(35 \%)$ cases, 2 ducts in 5(9\%) and $\geq 3$ ducts in $19(35 \%)$ cases. DCIS was diagnosed in $21(39 \%)$ and $23(43 \%)$ cases using $>90 \%$ proportion and $>3 \mathrm{~mm}$ linear extent criteria respectively. ADH was identified outside the papillary lesion in 17(31\%) cases; extra-lesional ADH was not associated with papillary lesions $<2 \mathrm{~mm}$. Papilloma size did not predict follow-up excision findings. Follow up excision was benign in 8/37 cases; ADH or carcinoma was present in 29/37 cases. The follow-up excision findings were significantly different with linear extent criteria $(p=.027)$ as compared to proportion criteria $(p=0.3)$ and were independent of the papillary lesion size and presence of extra-lesional ADH. UDH, sclerosis, and separation of the ducts by stromal tissue and cystic dilatation of the ducts hampered the assessment of both linear extent and proportion of atypia.

Conclusions: Both linear dimension and proportion criteria labeled a similar number of papillomas with atypia as DCIS; however the linear extent criteria did slightly better in predicting atypia in follow-up excisions. Atypia in follow up excisions was independent of papillary lesion size and presence of extra-lesional ADH. Assessment of atypia in a papilloma by linear extent or by proportion is equally complex.

185 Expression of Splicing Variants of Androgen Receptor in Primary and Metastatic Breast Cancer

Yehonatan Kane, Fang-Ming Deng, Yang Zhan, Yanfeng Qi, Jane D Hon, Xichun Liu, Haitao Zhang, Jinhua Wang, Rachel Brody, Rosemary Wieczorek, Yan Dong, Peng Lee, Baljit Singhb. New York University Langone Medical Center, New York, NY; Tulane University, New Orleans, MI

Background: The androgen receptor (AR) and its pathway have been implicated in tumorigenesis and progression of breast cancer. Anti-androgen therapy has shown efficacy in the metastatic breast cancer and numerous clinical trials are underway to study efficacy in various clinical settings. 15 splicing variants of AR (AR-Vs) have been described in prostate cancer. In prostate cancer, some of the AR-Vs especially AR-V7 are associated with aggressive disease and resistance to anti-AR therapy. The AR-V prevalence in human breast cancer specimens has hitherto not been studied. We 
aimed at studying the expression of AR-Vs in breast cancer specimens and present the data on AR-V1, AR-V7, AR8, and ARv567es, in AR-positive triple negative (TNBC), $\mathrm{ER}+/ \mathrm{Her} 2-$, and metastatic breast cancer.

Design: 98 cases of TNBC, 40 cases of ER+/Her2- primary breast cancer, 16 cases of metastatic cancer and 17 cases with reduction mammoplasty were analyzed for AR expression by immunohistochemistry (IHC). IHC for AR was performed using antibody clone N-20 (Santa Cruz) at 1:100 dilution. Normal breast tissue was used as internal control and $10 \%$ nuclear staining was used for categorizing a tumor as AR positive. A subset of cases which over-expressed AR were macrodissected from formalin fixed paraffin embedded sections with total RNA extracted by using the PureLink $\mathbb{R}$ FFPE RNA Isolation Kit (Invitrogen). Reverse-transcription was performed by using

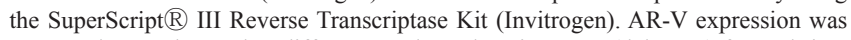
presented as cycle number difference to housekeeping gene (delta $\mathrm{CT}$ ) for real-time PCR or as absolute copy number for digital PCR.

Results: $\mathrm{AR}+\mathrm{TNBC}$ and $\mathrm{AR}+/ \mathrm{ER}+$ cases ranged from stage $1 \mathrm{~A}$ to IIIA. IHC for AR showed $>10 \%$ staining in 27 of $98 \mathrm{TNBC}$ cases and in 39 of $40 \mathrm{ER}+/ \mathrm{Her} 2$ - cases. ARV7 was expressed in 11 of $13 \mathrm{AR}+/ \mathrm{TNBC}$ cases $(\mathrm{p}<0.05)$; AR-V1 and AR-V4 were expressed in 4; ARV8 and ARv567es were expressed in 3 and 2 cases respectively. In $25 \mathrm{AR}+/ \mathrm{ER}+/$ Her2- cases AR-V7 was expressed in 14; AR-V4 in 9; ARv567es in 5, AR-V1 in 3 cases. Surprisingly, these AR-Vs are rarely expressed in metastatic breast cancer with AR-V7 expression in only one case and AR-V567 in one case of 16 cases. Full length AR is expressed in all cases as control.

Conclusions: We report expression of different various spliced variants in TNBC, ER+/ Her2- and metastatic breast cancer. A statistically significant expression of AR-V7 is seen in TNBC. AR-Vs are rarely expressed in metastatic breast cancer. AR-V status may predict breast cancer progression.

186 Does Tumor Infiltrating Lymphocyte (TIL) Count Influence Oncotype DxTM Recurrence Score and Magee Equation Scores?

Brie Kezlarian, Laura Favazza, Javier Arias-Stella, Daniel Schultz, Dhananjay A Chitale. Henry Ford Hospital, Detroit, MI.

Background: Genomic health Inc. -Oncotype Dx ${ }^{\mathrm{TM}}$ assay (GHI), a RT-PCR based genomic test analyzes the expression of 21 genes to give a distant breast cancer Recurrence Score (RS), stratifying ER+, node negative breast cancer patients for or against chemotherapy. The Magee equations (ME) use standard pathologic and immunohistochemical parameters [estrogen receptor (ER), progesterone receptor (PR), MIB1 proliferation index (PI) and tumor size] to estimate the RS. Proliferation group of genes are heavily weighted in most of these mathematical equations and correlates with PI. TILs generally have high PI. High TIL count is known to indicate good prognosis in breast cancer and is reported to predict sensitivity for chemotherapy. Our aim of this study was to investigate the correlation between TIL, PI, RS, and ME in estrogen receptor positive $(\mathrm{ER}+)$ invasive breast cancers $(\mathrm{BC})$.

Design: Cases of ER+BC that had GHI-RS results available were identified, tissue microarrays (TMAs) with a $1.0 \mathrm{~mm}$ diameter, each case represented in triplicate, were constructed. Multiple clinical and morphologic parameters including histologic features, hormonal status and TMN stage were recorded from electronic pathology report. TMAs were stained with different T lymphocyte markers (CD3, CD4, CD8) and leukocyte common antigen (LCA) to score all the lymphocytes. The average number of positive cells per case was counted. $\mathrm{ER}+\mathrm{BC}$ were subclassified as Luminal $\mathrm{A}$ (ER+/PR+/HER2-/Low MIB1), Luminal B (ER+/PR+/HER-2+ or high MIB1 labeling $>15 \%)$. Pearson correlation was performed to compare clinicopathologic parameters, GHI-RS and ME. Results: A total of 160 cases of breast carcinomas were identified. The average tumor size was $1.7 \mathrm{~cm}$ (SD: 1.0 , range: $0.4-8.5 \mathrm{~cm}$, median: $1.5 \mathrm{~cm}$ ). There were 52/160 $(32.5 \%)$ grade $1,88 / 160(55 \%)$ grade $2,20 / 160(12.5 \%)$ grade 3 tumors. There were 160/160 (100\%) ER+, 139/160 (87\%) PR+, 21/160 (13\%) PR-, 152/160 (95\%) HER2and $8 / 160(5 \%)$ HER $2+$ tumors. The GHI-RS included $94 / 160(59 \%)$ low RS, 59/160 (37\%) intermediate RS, 7/160 (4\%) high RS. Pearson correlation between TIL count vs GHI-RS \& ME were: GHI-RS : $0.169412(\mathrm{p}=0.0339), \mathrm{ME} 1=0.298713(\mathrm{p}=0.000226)$; ME2: $0.235647(\mathrm{p}=0.002974)$, ME3: $0.339557(\mathrm{p}<0.00002)$.

Conclusions: In this study, the high TIL count in $\mathrm{ER}+\mathrm{BC}$ was associated with an elevation of both GHI-RS and ME. More studies to support this observation are necessary for confirmation.

\section{Loss of PTEN in High Grade Advanced Stage Triple Negative Breast} Ductal Cancers in African American Women

Farhan Khan, Tammey Naab, Luisel J Ricks-Santi, Yasmine Kanaan, Girmay Asgedom. Howard University Hospital, Washington, DC; Hampton University, Hampton, VA. Background: PTEN is a tumor suppressor gene that inhibits cell proliferation by inhibiting phosphoinositide 3- kinase (PI3K) signaling pathway. The significance of PTEN mutations and their impact on prognosis of breast cancer is not well established. The objective of our study was to correlate the immunohistochemical expression of PTEN in the four major subtypes of breast carcinoma (Luminal A, Luminal B, HER2 positive, and Triple Negative) in a population of 202 African-American (AA) females with other clinicopathological factors including grade, stage, disease-free, and overall survival.

Design: Tissue microarrays (TMAs) were constructed from FFPE tumor blocks from primary ductal breast carcinomas in 202 African-American females. Two separate $1 \mathrm{~mm}$ cores represented each case. The polymer-HRP system was utilized for immunostaining. Five micrometer sections are stained with a mouse monoclonal antibody against PTEN (6H2.1, Dako). The sections were evaluated for the intensity of cytoplasmic and nuclear reactivity. Specifically, the cytoplasmic and/or nuclear immunoreaction was scored based on intensity whereby a score of 2 = positive (equal in intensity to normal epithelial cells), a score of 1 = weak (reduced intensity as compared with normal epithelial cells), and a score of $0=$ negative (no immunoreaction). Bivariate analysis was done via $\chi 2$ analysis and survivability data was calculated via the generation of Kaplan-Meier curves (SPSS v19). Statistical significance was assumed if $\mathrm{P}<0.05$.

Results: Loss of PTEN expression was associated with ER negative $(p=0.021)$, PR negative $(p=0.024)$ and triple negative $(p=0.0024)$ breast ductal cancers. It was marginally associated with distant metastasis $(\mathrm{p}=0.074)$. There was no association between PTEN loss and recurrence-free survival or overall survival.

Conclusions: In our study, a statistically significant association between PTEN loss and the triple negative breast cancers (TNBC)was found in AA women. PTEN loss was marginally associated with distant metastasis. PTEN inhibits PI3K resulting in decreased activation of downstream effector, mammalian target of rapamycin (mTOR). Loss of PTEN results in cell proliferation through activation of mTOR. Target therapy with $\mathrm{mTOR}$ inhibitors seems promising in the treatment of TNBC.

188 Inconsistency in Following the AJCC Reporting Guidelines for T-Staging of Breast Cancer with Skin Ulceration

Thaer Khoury, Rouzan G Karabakhtsian, Zaibo Li, Mohamed M Desouki, Souzan Sanati, Yisheng V Fang, Dan Wang, Carmelo Gaudioso, Rebecca Jacobson. Roswell Park, Buffalo, NY; Montefiore, Bronx, NY; Ohio State, Columbus, OH; Vanderbilt, Nashville, TN; Washington University, Saint Louis, MO; University Texas Southwest, Dallas, TX; University Pittsburgh Medical Center, Pittsburgh, PA.

Background: The AJCC staging for $\mathrm{pT}$ category in breast cancer $(\mathrm{BC})$ is based on tumor size (T1 to T3). pT4b is a unique entity which is defined as tumor with skin ulceration (SU) regardless of tumor size. The purpose of this study is to evaluate the pathologist adherence to the AJCC staging guidelines.

Design: We used mixed methods including a survey and archival data analysis. Sixtythree practicing pathologists from multiple institutions were surveyed on how these tumors $(8-\mathrm{mm}, 15-\mathrm{mm}, 30-\mathrm{mm}$ and $60-\mathrm{mm})$ with SU should be staged. A copy of the AJCC staging guidelines was provided to the participants at the time of the survey. The type of practice (academic vs. private), specialization in breast pathology, number of breast cases reviewed in a week, and the number of years practiced were recorded. Reports of BC cases $(n=111)$ with SU were pooled from 7 academic institutions. The tumor size, T-stage, and the lymph node stage were abstracted from the records. Results: The surveyed pathologists were $92 \%$ academic, $70 \%$ specialized in breast pathology, $56 \%$ review at least 10 cases a week, and $72 \%$ had at least 6 years of experience. The tumors measuring $8-\mathrm{mm}, 15-\mathrm{mm}, 30-\mathrm{mm}$ and $60-\mathrm{mm}$ were staged as pT4b by $90 \%, 92 \%, 95 \%$ and $95 \%$ of the participants, respectively. Although there was a trend with regard to tumor size, it was not statistically significant. For the reported cases $(\mathrm{n}=111)$ the mean (range) of tumor size was $58.51(2-186) \mathrm{mm}$. The tumors measured $\leq 20$-mm, 21-50-mm and $>50-\mathrm{mm}$ in $19(17.1 \%), 41(36.9 \%)$ and $51(46 \%)$ cases respectively. The reported T-stage was pT4b in $88(79.3 \%)$ cases and non-pT4b in 23 $(20.7 \%)$ cases. The tumors were staged as pT1 in 9 of $19(47.4 \%)$ cases that measured $\leq 20-\mathrm{mm}, \mathrm{pT} 2$ in 9 of $41(22 \%)$ cases that measured $21-50-\mathrm{mm}$, and pT3 in 5 of 51 $(9.8 \%)$ cases that measured $>50-\mathrm{mm}(\mathrm{p}=0.003)$. After excluding cases with stage IV $(n=21)$ and stage $X(n=10), 17$ of $80(21.3 \%)$ cases were incorrectly staged (6 patients IA; 4 IIA: 2 IIB and 5 IIIA) $(\mathrm{p}<0.0001)$. The pathologists tend to down-stage cases in the real life (reports) than in the survey $(\mathrm{p}<0.0001)$.

Conclusions: There is inconsistent adherence to the AJCC staging guidelines regarding staging BC with SU. The pathologist tends to down-stage a tumor with a small size. Studies are needed to investigate if small tumors with skin ulceration behave like pT4 tumors.

189 Skin Ulceration in Breast Cancer Carries No Increased Risk of Disease Free or Overall Survival

Thaer Khoury, Yisheng V Fang, Souzan Sanati, Mohamed M Desouki, Zaibo Li, Rouzan G Karabakhtsian, Dan Wang, Carmelo Gaudioso, Rebecca Jacobson. Roswell Park, Buffalo, NY; University Texas Southwest, Dallas, TX; Washington University, Saint Louis, MO; Vanderbilt, Nashville, TN; Ohio State, Columbus, OH; Montefiore, Bronx, NY; University Pittsburgh Medical Center, Pittsburgh, PA.

Background: The AJCC guidelines stage breast cancer (BC) with skin ulceration (SU) as pT4b (locally advanced disease) regardless of the tumor size. The purpose of this study is to evaluate if $\mathrm{SU}$ is an independent risk of survival.

Design: $\mathrm{BC}$ cases with $\mathrm{SU}(\mathrm{n}=27)$ from 7 academic institutions were included in this study (study cohort). The inclusion criteria are: no neoadjuvant treatment, not a recurrence, ulceration not due to biopsy site or infection, procedure not a small skin biopsy (shave or punch), and not clinically inflammatory carcinoma. These cases were matched with the following matching criteria: age (50 years cutoff), gender, histologic type, tumor size (2-20-mm, 21-50-mm), lymph node stage (0 to 3), molecular subtype [hormone receptor (HR)/HER2], margin status, chemotherapy (CT) including antiHER2 therapies, radiation therapy (RT) and hormonal therapy (HT). Disease free survival (DFS) and overall survival (OS) are the end points to compare between study cohort $v s$. matching cohorts.

Results: The mean and range of tumor size was 28.63 (2 to 50) $\mathrm{mm}$ for the study cohort and $24.11(5$ to 45$) \mathrm{mm}$ for the matching cohort. There were $8(30 \%)$ cases with tumor size $\leq 20-\mathrm{mm}$, and $19(70 \%)$ with tumor size 21-50-mm. Pathology-node stage was $\mathrm{pN} 0$ in $6(22 \%)$, pN1mi in $1(4 \%)$, pN1 in $9(33 \%)$, pN2 in $8(30 \%)$, and pN3 in $3(11 \%)$ cases. There were 17 (63\%) HR+/HER2- cases, $6(22 \%)$ HER2+, and $4(15 \%)$ triple negative. The number of patients who had CT, anti-HER2 therapy, RT and HT was 14 $(52 \%), 5(19 \%), 14(52 \%)$, and $19(70 \%)$, respectively. While $6(22 \%)$ patients died of disease in the study cohort, $8(30 \%)$ died in the matching cohort. While $10(37 \%)$ patients developed local recurrence in the study cohort, $6(22 \%)$ developed local recurrence in the matching cohort. There was no statistical significant difference in the DFS or OS 
between the two cohorts. The 5-year DFS probability was $61 \%$ for the study cohort and $65 \%$ for the matching cohort; and for OS $77 \%$ for the study cohort and $83 \%$ for the matching cohort with no statistical significant difference.

Conclusions: Skin ulceration in $\mathrm{BC}$ may not be an independent factor to predict a more aggressive clinical behavior. Therefore, the AJCC staging system should be interpreted with caution and more studies with larger number of cases are needed to validate our findings.

\section{Breast Cancer with Skin Ulceration: Should All Cases Be Staged as pT4b?}

Thaer Khoury, Souzan Sanati, Zaibo Li, Yisheng V Fang, Mohamed M Desouki, Rouzan G Karabakhtsian, Dan Wang, Carmelo Gaudioso, Rebecca Jacobson. Roswell Park, Buffalo, NY; Washington University, Saint Louis, MO; Ohio State University, Columbus, OH; University Texas Southwest, Dallas, TX; Vanderbilt, Nashville, TN; Montefiore, Bronx, NY; University Pittsburgh Medical Center, Pittsburgh, PA. Background: Breast cancer (BC) with skin ulceration (SU) is considered by AJCC a locally advanced disease (stage III). The purpose of the study is to investigate if tumor size plays a role in the tumor behavior.

Design: BC cases with SU ( $=111)$ from 7 academic institutions were included in this study. These patients were treated in the adjuvant setting and had no clinical inflammatory carcinoma. Disease free survival (DFS) and overall survival (OS) were correlated with the tumor size (T1, T2, and T3) for all patients, then after excluding patients with stage IV disease. Then, we staged the patients as if there was no SU into stage I, II or III following the AJCC guidelines for the tumor size (T1, T2, T3) and node status (N0, N1, N2, N3). Finally, when all cases were T-staged as pT4b, they fell into two staging categories, either IIIB or IIIC.

Results: The mean (range) of tumor size was $58.5(2,186) \mathrm{mm}$ with $19(17.1 \%)$ tumors measuring $\leq 20-\mathrm{mm}$ and $41(36.9 \%) 21-\mathrm{mm}$ to $50-\mathrm{mm}$. There were $21(18.9 \%)$ patients with stage IV disease. Patients with larger tumor tended to present with distant metastases more often than patients with smaller tumors [median (range) $70(25,180)$-mm vs. 40 $(2,186)-\mathrm{mm}$, respectively $(\mathrm{p}=0.004)]$. When the tumors were staged as T1, T2 or T3, patients with larger tumors had worse OS $(\mathrm{p}=0.009)$ and worse DFS with borderline significance $(\mathrm{p}=0.067)$. When stage-IV cases were excluded, tumor size did not correlate with DFS ( $\mathrm{p}=0.8$ ) or OS ( $\mathrm{p}=0.165)$. However, when the cases (excluding stage IV) were staged based on tumor size (as if there was no SU) and node status into stage I, II or III, the OS was statistically significant $(\mathrm{p}=0.047)$ but not the DFS $(\mathrm{p}=0.195)$. When patients were staged as IIIB $v$. IIIC, the later had worse DFS $(\mathrm{p}=0.012)$, but not OS $(\mathrm{p}=0.265)$. None of the patients $(\mathrm{n}=12)$ who had tumor size $<18$-mm died from disease. Patients who had multifocal disease had 2.17 times higher risk of dying from disease than patients with a single focus $(\mathrm{p}=0.036)$.

Conclusions: Larger tumors with SU tend to present with distant metastases more often then small tumors. Small tumors had an extent of disease and overall survival similar to that observed in patients with early stages (stage I-II) disease. Therefore, it may be indicated to stage small tumors with SU in a staging category different from pT4b. However, more cases are needed to validate our findings.

191 Two Scoring Systems of Tumor Infiltrating Lymphocytes in Breast Cancer: Evaluating Inter-Observer Variability and TILs Heterogeneity Thaer Khoury, Vidya Nagrale, Dan Wang, Fadi Habib. Roswell Park, Buffalo, NY. Background: The goals of this study are to investigate 1) the inter-observer variability for tumor infiltrating lymphocytes (TILs) in breast cancer (BC); 2) if a single full section (FS) is enough to score for BC treated in the adjuvant setting through evaluating TILs heterogeneity between two FSs; and 3 ) the validity of scoring core biopsy (CB) in $\mathrm{BC}$ treated with neoadjuvant therapy through examining TILs heterogeneity between $\mathrm{CB}$ and $\mathrm{FS}$

Design: Matching CBs and two FSs (FS-1 and FS-2) of BC cases $(n=100)$ were independently reviewed by two pathologists. TILs were scored using two different scoring systems: intra-tumoral lymphocytes (iTu-Ly) and percentage of stromal lymphocytes (str-Ly). iTu-Ly was scored semi-quantitatively. str-Ly was scored following the recommendations of the International TILs Working Group 2014. To evaluate TILs heterogeneity the average scores of both observers were compared between both FSs. To assess the validity of scoring $\mathrm{CB}$, the average scores of CBs were compared to the average scores of both FSs among both observers. We evaluated if the number of cores, size of the tumor in the core or degree of necrosis had any effect on the concordance with the FSs. For practical reasons, cutoffs were arbitrary chosen to separate TILs into three classes, no lymphocytes, focal lymphocytes and predominant lymphocyte $\mathrm{BC}$

Results: The weighted kappa values for the degree of agreement between both observers across all samples were substantial for str-Ly and moderate for iTu-Ly. The weighted kappa values for both str-Ly and iTu-Ly was almost perfect when the cases were grouped into three classes. TILs Pearson correlation between FS-1 and FS-2 was $93 \%$ for iTuLy and $91 \%$ for str-Ly $(\mathrm{p}<0.0001)$. The number of cases that had agreement in TILs classes between FS-1 and FS-2 was $84 \%$ for iTu-Ly and $79 \%$ for str-Ly $(p<0.0001)$. The CB scores reflected the average FSs with Pearson correlation of $84 \%$ for iTu-Ly and $86 \%$ for str-Ly $(\mathrm{p}<0.0001)$. The number of cases that had agreement in TILs classes between CB and average FSs was $78 \%$ for iTu-Ly and $69 \%$ for str-Ly $(p<0.0001)$. The multivariate linear regression analysis showed that CB and average FSs were still significantly correlated after adjusting the number of cores, size of the tumor in the core and degree of necrosis $(\mathrm{p}<0.0001)$

Conclusions: The inter-observer variability of scoring TILs might not be an issue regardless of TILs location. Due to TILs heterogeneity, scoring two FS might be better than scoring a single FS. TILs scores in CB should be interpreted with caution, as the class of up to one-third of the cases may be misclassified
192 Prognostic Significance of Stromal Versus Intratumoral Infiltrating Lymphocytes in Different Molecular Subtypes of Breast Cancer Treated with Neoadjuvant Therapy

Thaer Khoury, Vidya Nagrale, Xuan Peng, Mateusz Opyrchal, Dan Wang, Song Yao. Roswell Park, Buffalo, NY.

Background: It has been shown that tumor infiltrating lymphocytes (TILs) predict pathologic complete response (pCR) in HER2 + and triple negative breast cancer (TNBC) treated with neoadjuvant therapy (NAT). Achieving pCR has been shown to portend better prognosis in these subtypes. We hypothesize that tumor-immune interactions adds another layer of tumor heterogeneity across tumor molecular subtypes. To test this hypothesis, we investigated if there are differences in associations of TILs with pCR among BC molecular subtypes and if TILs location [intra-tumoral (iTu-Ly) vs. stromal (str-Ly)] differentially predicts pCR in patients treated with NAT.

Design: Hematoxylin and Eosin slides of BC core biopsy cases $(n=355)$ were reviewed from a single institution between 2000 and 2014. str-Ly was scored following the International TILs Working Group 2014 recommendations ranging from $0 \%$ to $100 \%$. iTu-Ly was scored semi-quantitatively incorporating the infiltrate grade ( 0 to 3 ) and the corresponding percentage resulting in a score ranging from 0 to 300 . Key clinicopathologic variables were recorded. pCR was defined as no residual infiltrating tumor in the tumor bed and the lymph nodes.

Results: pCR was achieved in 29 of 95 (30.5\%) TNBC cases, 25 of $77(32.5 \%)$ HER2+, and 9 of $183(4.9 \%)$ luminal/HER2-. In univariate analysis, younger age, invasive nonlobular carcinoma, higher Nottingham grade, non-luminal/HER2- subtype, Herceptin therapy, str-Ly, and iTu-Ly predicted pCR. In multivariate analysis of all cases, both str-Ly and non-luminal/HER2- subtype were independent predictors of $\mathrm{pCR}$ ( $\mathrm{p}<0.0001$ and 0.0004 , respectively). Similarly, both iTu-Ly and non-luminal/HER2- subtype also independently predicted $\mathrm{pCR}$ ( $\mathrm{p}=0.0008$ and 0.0009 , respectively). In multivariate analysis within each of the molecular subtype groups, iTu-Ly $(\mathrm{p}=0.0053)$ but not str-Ly $(\mathrm{p}=0.062)$ predicted $\mathrm{pCR}$ in the luminal/HER2- subtype. In the TNBC subtype, both iTu-Ly and str-Ly predicted pCR, with str-Ly being a stronger predictor ( $\mathrm{p}=$ 0.009 and 0.0004 , respectively). In the HER $2+$ subtype, only Herceptin treatment was statistically significant in predicting pCR, where 25 of $68(36.8 \%)$ treated patients had $\mathrm{pCR}(\mathrm{p}=0.027)$; however, neither str-Ly nor iTu-Ly predicted pCR $(\mathrm{p}>0.05)$.

Conclusions: TILs might be functionally heterogeneous with regard to their role in mediating anti-tumor immune response, depending on their location and BC molecular subtypes. Our results suggest that TILs are predictive of pCR in the luminal/HER2- and the TNBC subtypes, but not in the HER2+ subtype.

193 Comprehensive Evaluation of Ki67 in Luminal/HER2-Negative Breast Cancer in the AMBER Consortium

Thaer Khoury, Gary Zirpoli, Stephanie M Cohen, Mihai Merzianu, Melissa Troester, Joseph Geradts, Amber Worral, Wiam Bshara, Christine Ambrosone. Roswell Park, Buffalo, NY; University North Carolina, Chapel Hill, NC.

Background: The goal of this study was to comprehensively evaluate $\mathrm{Ki}-67$ index in luminal/HER2- breast cancer (BC) for potential use of automated image analysis and scoring in a large epidemiologic study.

Design: Tissue microarrays (TMA) (two 1-mm cores and three 0.6-mm) were constructed from 110 BC. Full sections (FS) and TMA slides were stained with Ki-67 (MIB1, DAKO). Image analysis (Aperio) was performed using the Genie algorithm that enriched for epithelial cells in five layers: FS (around the tumor), tumor/stroma interface $(\mathrm{InF})(\mathrm{n}=108)(0.5-\mathrm{mm}$ thick), hot spot (HS) $(\mathrm{n}=52)$ (visually identified), ductal carcinoma in situ (DCIS) $(n=55)$ and aggregated lymphocytes $(n=74) .14 \%$ cutoff was arbitrarily chosen to separate luminal A from luminal B. Ki-67 in FS was compared with all other layers, with $1-\mathrm{mm}$ core and $0.6-\mathrm{mm}$ core. Comparison analysis for cases that had FS and all TMA cores $(n=49)$ was performed. We assessed the best way of collapsing cores in order to better reflect the FS. We tested two methods, one using tumor cellularity weighted approach and the other classifying a case as luminal $\mathrm{B}$ if any core had $\mathrm{Ki}-67$ index $>14 \%$

Results: The median (range) of Ki-67 index (\%) was 8.2 (0.56 to 28.3) for FS, 6.4 (0.21 to 32.9) for DCIS, 9.7 (0.48 to 40.3$)$ for InF, 18.6 (3.7 to 46.6$)$ for HS, and 4 (0 to 9.9 ) for lymphocytes $(\mathrm{p}<0.0001)$. Compared to FS and using $14 \%$ cutoff, DCIS had higher Ki-67 index in 3 of $55(5.5 \%)$ cases and lower in $6(10.9 \%)$; InF had higher in 10 of $108(9.3 \%)$ cases and lower in $1(0.9 \%)$; HS had higher in 23 of $52(44.2 \%)$ cases and lower in $0 \%$; and lymphocytes had higher in 0 of $74(0 \%)$ cases and lower in $14(18.9 \%)$. For cases with at least a single $0.6-\mathrm{mm}$ TMA core $(\mathrm{n}=107), 15 \%$ were misclassified (luminal A vs. luminal B) compared to FS using 14\% cutoff approach and $10.3 \%$ using weighted approach. For cases with at least a single 1-mm TMA core $(\mathrm{n}=101), 5 \%$ were misclassified compared to FS using either approach. For cases with both 1.0-mm TMA cores, $8.2 \%$ were misclassified using $14 \%$ cutoff approach and $10 \%$ using weighted approach. For cases that had all three $0.6-\mathrm{mm}$ TMA cores, $14.3 \%$ were misclassified using $14 \%$ cutoff approach and $12.2 \%$ using weighted approach. Subtracting lymphocytes and DCIS from FS had no effect on the comparison between the FS and TMA cores

Conclusions: Hot spots are common in luminal type BC. InF tends to have higher Ki-67 index. Scoring HS or InF skews the Ki-67 index to the high end. Using at least $1.0-\mathrm{mm}$ core had the lowest discordance with the FS. DCIS and lymphocytes did not have effects on the Ki-67 index of FS 
194 Negative Association between GATA3 and Fascin Could Predict Relapse-Free and Overall Survival in Patients with Breast Cancer

Dong-Hoon Kim, Kyueng-Whan Min, Sung-Im Do, Seoung Wan Chae, Jin Hee Sohn. Kangbuk Samsung Hospital, Sungkyunkwan University School of Medicine, Seoul, Republic of Korea; Hallym University Sacred Heart Hospital, Hallym University College of Medicine, Anyang, Gyeonggi-do, Republic of Korea.

Background: GATA3 and fascin proteins are known prognostic markers in several cancers. GATA3 is a key regulator of mammary-gland morphogenesis and luminal-cell differentiation, whereas fascin is a pro-metastatic actin bundling protein.

Design: In this study, we analyzed and compared the predictive abilities of GATA3 and fascin for clinical outcomes of patients with breast cancer. The combined expression pattern based on GATA3-/+ and fascin-/+ was evaluated by immunostaining using a tissue microarray, and relationships between protein expression and several clinicopathological parameters were analyzed.

Results: GATA3 expression was associated with good prognostic parameters, but fascin was correlated with poor prognostic parameters. On comparing GATA3 and fascin, we found an inverse relationship between fascin and GATA3 expression. On analysis of combined markers, GATA3+/fascin- was correlated with improved clinical outcomes compared to GATA3-/fascin+. Univariate and multivariate analyses revealed significant differences in relapse-free and overall survival between GATA3+/fascinand GATA3-/fascin+

\begin{tabular}{|c|c|c|c|c|}
\hline Relapse-free survival & $\begin{array}{l}\text { Univariate } \\
\text { significance }^{1}\end{array}$ & $\begin{array}{l}\text { Multivariate } \\
\text { significance }^{2}\end{array}$ & $\begin{array}{l}\text { Relative } \\
\text { risk }\end{array}$ & $95 \% \mathrm{CI}$ \\
\hline GATA3+/fascin- vs. GATA3-/fascin+ & 0.031 & 0.026 & 5.343 & $1.2-23.4$ \\
\hline GATA3+/fascin- $v s$. GATA3-/fascin- & 0.032 & 0.163 & 1.306 & $0.9-1.9$ \\
\hline GATA3-/fascin- $v s$. GATA3-/fascin+ & 0.584 & 0.265 & 1.605 & $0.9-3.7$ \\
\hline GATA3+/fascin- $v s$. GATA3+/fascin + & 0.709 & 0.875 & 1.066 & $0.48-2.35$ \\
\hline GATA3+/fascin+ $v s$. GATA3-/fascin + & 0.609 & 0.472 & 2.424 & $0.22-27.1$ \\
\hline GATA3-/fascin- $v s$. GATA3+/fascin+ & 0.731 & 0.688 & 0.655 & $0.08-5.19$ \\
\hline \multicolumn{5}{|l|}{ Overall survival } \\
\hline GATA3+/fascin- vs. GATA3-/fascin+ & 0.002 & 0.032 & 5.107 & $1.16-22.58$ \\
\hline GATA3+/fascin- $v s$. GATA3-/fascin- & 0.121 & 0.483 & 1.174 & $0.75-1.84$ \\
\hline GATA3-/fascin- vs. GATA3-/fascin+ & 0.084 & 0.116 & 1.985 & $0.85-4.67$ \\
\hline GATA3+/fascin- vs. GATA3+/fascin+ & 0.421 & 0.914 & 0.953 & $0.4-2.28$ \\
\hline GATA3+/fascin $+v s$. GATA3-/fascin + & 0.577 & 0.807 & 1.322 & $0.14-12.36$ \\
\hline GATA3-/fascin- $v s$. GATA3+/fascin+ & 0.908 & 0.362 & 0.37 & $0.04-3.13$ \\
\hline
\end{tabular}

Conclusions: Combined marker analysis of GATA3/fascin showed an inverse association and improved prognostic information for patients with breast cancer.

195 A Novel Risk-Predictive Metric Stratifies Triple-Negative Breast Cancers Based on Cell Cycling Kinetics

Sergey Klimov, Andrew Green, Mohammed A Aleskandarany, Emad Rakha, Ian O Ellis, Guilherme Cantuaria, Ayodeji O Agboola, Michelle Reid, Xiaoxian Li, Padmashree CG Rida, Ritu Aneja. Georgia State University, Atlanta, GA; University of Nottingham, Nottingham, United Kingdom; Northside Hospital Cancer Institute, Atlanta, GA; Olabisi Onabanjo University, Sagamu, Nigeria; Emory University, Atlanta, GA.

Background: Ki67 Index (KI) and mitotic index (MI) are proliferation markers with established prognostic value in breast cancer. These indices are evaluated individually and on disparate measurement scales; they therefore fail to capture information about cell cycling kinetics of proliferating cells.

Design: Pathology reports of breast cancer patients ( $\mathrm{n}=10,504$ from Northside Hospital, Atlanta and $\mathrm{n}=1560$ from Nottingham Hospital, UK) were retrospectively analyzed for mitotic scores, $\mathrm{KI}$ and clinical outcomes. $267 \mathrm{H} \& \mathrm{E}-$ stained breast carcinoma samples were analyzed by two pathologists, to transform mitotic scores from a range to an average, and then into \%MI based on cellularity. \%MI: \% $\mathrm{KI}$ ratio was defined as the Ki67-Adjusted Mitotic Score (KAMS), which reflects cycling kinetics of proliferative cells. Ability of KAMS to stratify triple-negative breast cancers (TNBCs) was tested in three cohorts who received only adjuvant chemotherapy $(n=478$ from Northside Hospital, USA; $n=322$ from Nottingham Hospital, UK, and $n=108$ from Olabisi Onabanjo University, Nigeria). Kaplan-Meier survival analysis, $\mathrm{c}^{2}$ values, multivariate analyses and model fit statistics were used to compare stratification yielded by KAMS, $\mathrm{KI}$ and MI. Slow-cycling and fast-cycling TNBC subgroups from Nottingham Hospital were analyzed for biomarker expression.

Results: KAMS stratified TNBCs into two subgroups significantly different in outcomes. This stratification was superior to that by either KI or MI, regardless of hospital, and KAMS retained its significance in multivariate analyses. Fast-cycling TNBCs have poorer prognosis than slow-cycling TNBCs, perhaps due to higher intratumoral heterogeneity in fast cycling tumors. Fast-cycling TNBCs showed high expression of proteins implicated in DNA damage response, sumoylation, EGFR and Androgen Receptor signaling, and metastasis. By contrast, slow-cycling TNBCs showed extensive chromatin modification.

Conclusions: KAMS quantifies cell cycling kinetics, stratifies TNBCs and yields new risk-predictive information that is not revealed by either KI or MI. KAMS reveals the underlying heterogeneity in cycling kinetics among TNBCs and helps identify TNBCs who might benefit from treatments that target the cell cycle machinery.
196 Predicting Metastatic Site of Triple Negative Breast Cancers Using Immunohistochemical Biomarkers

Sergey Klimov, Andrew Green, Mohammed A Aleskandarany, Emad Rakha, Ian O Ellis, Michelle Reid, Padmashree CG Rida, Ritu Aneja. Georgia State University, Atlanta, GA; University of Nottingham, Nottingham, United Kingdom; Emory University, Atlanta, GA, United Kingdom.

Background: Unique organ microenvironment may preferentially support growth of specific tumor clones because of which different breast cancer subtypes show distinct tropisms for sites of metastasis. While a few gene expression-based signatures are known to predict site-specific metastasis of breast cancer, little work has focused on identification of clinically facile immunohistochemical predictors of metastasis to specific sites, especially for triple negative breast cancers (TNBCs).

Design: Primary tumor samples from 322 TNBC patients diagnosed between 1987 and 1998 were stained for 133 biomarkers and assessed by immunohistochemistry. Differences in average levels of these biomarkers were compared between patients with metastasis to a specific site (brain, bone, lungs, liver, lymph nodes), and those that did not show metastasis to that site. Significantly different biomarkers were analyzed within a Cox regression model to evaluate their prognostic value when patients with metastasis to the site of interest were compared to patients with no metastasis. Ideal thresholds of prognostic markers were then found, which maximized stratification based on time-to-metastasis to the specific sites, between cohorts that show high and low expression of each biomarker

Results: Our analysis uncovered several biomarkers ( 8 for bone, 4 for brain, 8 for lung, 8 for liver, and 11 for lymph node) whose expression levels in primary tumors can predict the site of future metastasis in TNBCs. The variables which provided the largest significant $(\mathrm{p}<0.01)$ change in hazard for a specific site included a low Adjacent CD8 for bone (Hazard Ratio $=3.642$ ), high Poly (ADP-ribose) polymerase-1 (PARP1) $\%(\mathrm{HR}=4.35)$ for brain, retinoid-related orphan receptor- $\gamma$ cytoplasmic $\mathrm{H}$-score $(\mathrm{HR}=$ $5.292)$ for lung, high trefoil protein TFF1 \% $(\mathrm{HR}=2.194)$ for liver, and a low Vitamin D Receptor Nuclear H-score $(\mathrm{HR}=8.767)$ for lymph node. When tested using the whole dataset, (a) biomarkers that predicted metastasis to liver and brain showed sensitivity $>0.75$, and (b) biomarkers that predicted metastasis to liver and lung, showed specificity $>0.75$, respectively.

Conclusions: Relatively simple and inexpensive immunohistochemical analyses of biomarkers in primary tumors of TNBCs may allow prediction of the site of future metastasis. High-risk patients may benefit from increased surveillance of such sites.

197 Reproducible Evaluation of Tumor-Infiltrating Lymphocytes (TILs) Using the Recommendations of International TILs Working Group 2014 Yumi Kojima, Frances Compton, Hongxia Sun, Xiaohong I Wang, Michael Covinsky, Songlin Zhang. University of Texas Health Science Center at Houston, Houston, TX. Background: Over the recent years, results from different groups have shown tumorinfiltrating lymphocytes (TILs) to have both prognostic and predictive value in breast cancer. In the BIG(Breast International Group) 2-98 study, high TILs ( $>50 \%$ ) in operable, triple negative breast cancer was associated with favorable disease free and overall survival, and every $10 \%$ increase of TILs was associated with $17 \%$ reduction in the risk of relapse and death. A standardized methodology for evaluating TILs is necessary for integrating this parameter in daily practice, and recommendations and instructions by an International TILs Working Group 2014 have recently been published in Annals of Oncology (2015). The goal of our current study is to investigate the consistency and reproducibility of these recommendations among pathology trainees and pathologists. Design: The study was approved by our Institutional Review Board, and 129 cases from 2009 to 2014 were selected for evaluation. One Hematoxylin and Eosin stained slide with tumor tissue was selected from each case based on the recommendations outlined by the International TILs Working group 2014. TILs were evaluated at an interval of $10 \% .3$ pathology trainees (PGY2, PGY3 and PGY4) and 3 pathologists (2 general pathologists and 1 breast pathologist) were involved in this study. The recommendations and instructions were reviewed by each person, and a group review of 5 selected cases was performed prior to the individual evaluation. Each case was than independently evaluated by 2 observers.

Results: Our results showed that in 107 cases $(107 / 129,82.9 \%)$ the TILs percentage was the same between the 2 observers. In 16 cases $(107 / 129,12.4 \%)$, the difference between the two observers was by $10 \%$ and in 6 cases $(107 / 129,4.7 \%)$ there was a difference of $20 \%$. There were no discrepancies of greater than $20 \%$ between the two observers. In our study set, we reported 10 cases with TILs greater than 50\% (10/129, $7.8 \%$ ). The majority of our cases (90/129) had less than $10 \%$ of TILs and 29 cases had TILs ranging from $10-50 \%$

Conclusions: Our study shows that the recommendations and instructions for TILs evaluation set by the International TILs Working Group 2014 are sufficiently detailed to be uniformly applied for generating reproducible TILs evaluation in breast cancer in the daily practice. Our results showed that in $95 \%$ of the time, the two observer outcome only differed by less than $10 \%$.

198 Establishing Recommendations for Her2 Staining in Cytology Cell Block Material

Fumiko Konno, Nyasha Bullock, Elyn Riedel, Gitika Aggarwal, Muzaffar Akram, Marcia Edelweiss. MSKCC, New York, NY.

Background: Her2 overexpression in breast carcinoma (ca) guides adjuvant anti-Her2 therapy. Immunohistochemistry (IHC) is one of the current methods in determining the Her2 status. ASCO/CAP guidelines of Her2 IHC are well established for surgical biopsy (Bx), but recommendations have not been made for cytology cell block (CB) material. Design: $\mathrm{Bx}$ with known $\mathrm{Her} 2$ status of invasive ca measuring $>1 \mathrm{~cm}$ with subsequent excision were studied. 8 cytology scrapings and $1 \mathrm{Bx}$ were performed on each ca at 
the time of grossing. Cytology scrapings were placed in $10 \%$ neutral buffered formalin (NBF) and processed after 1, 3, 6, 8, 12, 24, 48 and $72 \mathrm{~h}$, respectively. CBs were prepared as per protocol. Paired Bxs were placed in $10 \% \mathrm{NBF}$ for $8 \mathrm{~h}$. Histological processing included a short cycle with no NBF fixation step. H\&E-stained slides were prepared from CBs and Bxs. Slides were evaluated for cellularity $(0,<20$ or $>20$ cells) prior to IHC for Her2. Her2 scoring was recorded. Sensitivity (SEN) based on cytology results is estimated at each time point using biopsy results as the gold standard. SEN estimates are presented with exact $95 \%$ confidence intervals (CI).

Results: Ten cases of Her2 $3+$ invasive ca measuring $>1 \mathrm{~cm}$ were prospectively identified. Two patients (pts) underwent mastectomy and 8 pts had an excision over 14-months (9/2012-11/2013). Pts were female with mean age 58.9 (range 34 - 81). Mean tumor size was $2.3 \mathrm{~cm}$ (range $1.2-2.9 \mathrm{~cm}$ ). Final pathology diagnoses were all invasive ductal ca. The total number of CBs that met cellularity inclusion criteria was 56 (mean 5.6 and range 3-7 per time point). Number of Bxs that met inclusion criteria was 8 .

\begin{tabular}{|l|l|l|}
\hline Time point & $\#(3+)$ Positive/Total & Sensitivity $(95 \% \mathrm{CI})$ \\
\hline 1 & $5 / 8$ & $63 \%(24 \%-91 \%)$ \\
\hline 3 & $3 / 6$ & $50 \%(12 \%-88 \%)$ \\
\hline 6 & $5 / 7$ & $71 \%(29 \%-96 \%)$ \\
\hline 8 & $4 / 7$ & $57 \%(18 \%-90 \%)$ \\
\hline 12 & $6 / 9$ & $67 \%(29 \%-93 \%)$ \\
\hline 24 & $3 / 5$ & $60 \%(15 \%-95 \%)$ \\
\hline 48 & $2 / 7$ & $29 \%(4 \%-71 \%)$ \\
\hline 72 & $6 / 7$ & $86 \%(42 \%-99 \%)$ \\
\hline
\end{tabular}

Overall, 34/56 CBs $(61 \%)$ had $3+$ staining. Results at $72 \mathrm{~h}$ have the highest SEN. SEN in every other time point has an estimate in the range of $50 \%-71 \%$ except at $48 \mathrm{~h}$. For the cases fixed for less than $6 \mathrm{~h}$ (at 1 or $3 \mathrm{~h}$ ), all cases showed either $3+(8 / 14,57 \%)$ or $2+(6 / 14,43 \%)$ staining. In comparison, the $6-72 \mathrm{~h}$ CBs showed $3+$ in $26 / 42,62 \% ; 2+$ in $10 / 42,24 \%$; and $0 / 1+$ in $6 / 42,14 \%$ of the cases.

Conclusions: $\mathrm{CB}$ material fixed in NBF for as little as $1 \mathrm{~h}$ and as much as $72 \mathrm{~h}$ can be used to assess Her2 staining by IHC. The negative results found in well established time frames (6-72h) could be explained by pre-analytical variables such as an artifact introduced by the collection method (scraping vs actual FNA).

199 Feasibility of Using Confocal Fluorescence Microscopy for Imaging Breast Tissue in Surgical Pathology Practice

Savitri Krishnamurthy, Andrea Cortes, Mirtha Lopez, Michael Wallace, Sharjeel Sabir, Gordon Mills, Kenna Shaw. MD Anderson Cancer Center, Houston, TX.

Background: Optical imaging techniques are currently available for imaging tissues without the need for any special type of preparation. We studied the feasibility of a confocal fluorescence microscopy platform (Vivascope 2500, Caliber Inc. Rochester $\mathrm{NY}$ ) for examination of breast tissue.

Design: Fragments of breast tissue $(0.5-1.0 \mathrm{~cm}$ in maximum dimension) or 18 gauge core needle biopsies (CNBs) were procured from breast surgical resections. The tissues were stained with $0.6 \mathrm{mM}$ acridine orange for 6 seconds and imaged using the confocal microscopy platform with illumination of the tissues using a $400 \mathrm{~nm}$ laser. Mosaics of gray scale confocal fluorescent images were acquired after scanning through the tissue. The images were studied at different magnifications to obtain a primary diagnosis. The imaged tissue was then fixed in formalin to generate hematoxylin and eosin (H\&E) stained tissue sections for histopathological examination. The sensitivity and specificity of CFM for the interpretation of breast tissues was determined using the histological diagnosis as the gold standard.

Results: We imaged 38 breast specimens ( 32 tissue fragments and 6 CNBs). Mosaics of confocal fluorescent images of breast tissue fragments were obtained in 5-8 min and that of the CNBs in 3-5 min. The interpretation of CFM mosaics including zooming through the mosaics to visualize higher magnification to arrive at a primary diagnosis was achieved in $3 \mathrm{sec}$ to $5 \mathrm{~min}$ (mean: $1 \mathrm{~min}$ ) and for CNBs in $3 \mathrm{sec}$. The breast tissue was categorized as normal in 16 and malignant in 22 specimens: 18 cases of invasive ductal carcinoma (IDC), 2 invasive lobular carcinoma (ILC) and 2 ductal carcinoma in situ (DCIS). While the categorization of the breast tissues as benign or malignant was the same for CFM diagnosis, 2 cases of DCIS was incorrectly categorized as IDC on CFM. The overall sensitivity and specificity of CFM for the distinction of benign vs malignant categorization of the tissue when compared with the gold standard was $100 \%$ with a kappa coefficient of $0.5(95 \% \mathrm{CI} ; 0.08$ to 0.92$)$ for the agreement of specific tissue typing between the two platforms.

Conclusions: 1 . The relative ease and speed of image acquisition together with the quality of images and the high sensitivity and specificity of the diagnosis made possible from CFM images suggests that this platform has an excellent potential for rapid examination of breast tissue.

2. Prospective studies are warranted to establish the role of CFM as a robust, reproductive and reliable platform for rapid examination of breast tissue in surgical pathology practice.

\section{High Peripheral Stromal Lymphocytic Infiltration Is Associated} with Better Survival in Triple Negative Breast Carcinoma

Uma Krishnamurti, Ceyda Sonmez, Jing Yang, Limin Peng, Xiaoxian Li, Ritu Aneja. Emory University, Atlanta, GA; Georgia State University, Atlanta, GA.

Background: Tumor infiltrating lymphocytes (TIL) are associated with better response to neoadjuvant therapy and improved survival in breast carcinoma. However, the role of TIL in breast carcinoma without neoadjuvant therapy is not clear.

Design: TIL was evaluated in 157 triple negative (TNBC) and 187 estrogen receptor positive $(\mathrm{ER}+)$ breast carcinoma. TIL was defined as percentage of stroma occupied by infiltrating lymphocytes. TIL at the tumor periphery (peripheral TIL) as well as overall
TIL combining both TIL the periphery of and within the tumor were evaluated. TIL was correlated with lymphovascular invasion (LVI), tumor grade, stage, LN metastasis, and overall survival (OS) in TNBC and ER+ carcinoma and additionally with Oncotype DX scores in ER+ carcinoma.

Results: TIL was more noticeable at the tumor periphery in both ER+ and TNBC. In ER+ carcinoma, both peripheral and overall TIL were positively associated with Oncotype DX recurrence score ( $\mathrm{P}=0.0119$ and $\mathrm{P}=0.0007$ respectively). Only peripheral TIL was significantly associated with tumor grade $(\mathrm{P}=0.0184)$ in $\mathrm{ER}+$ carcinoma, with no association between TIL and OS observed. In TNBC, both peripheral and overall TIL were significantly associated with tumor grade $(\mathrm{P}<.0001$ and $\mathrm{P}=0.0004$ respectively), with higher TIL tending to have higher grade. Peripheral but not overall TIL was significantly associated with $\mathrm{OS}(\mathrm{P}=0.0338)$ and $\mathrm{DFS}(\mathrm{p}=0.0132)$ in TNBC, with higher peripheral TIL tending to have better OS and DFS.

Conclusions: Evaluating peripheral TIL is more relevant than overall TIL in breast carcinoma. Higher peripheral TIL is associated with better OS in TNBC and its role in $\mathrm{ER}+$ breast carcinoma needs further investigation.

201 Distant Metastases in Breast Cancer Patients with Oncotype Dx Recurrence Score Lower Than 18

Melissa Krystel-Whittemore, Edi Brogi, Zenica L Bowser, Maura Dickler, Clifford Hudis, Hannah Y Wen. The University of Kansas Medical Center, Kansas City, KS; Memorial Sloan Kettering Cancer Center, New York, NY.

Background: Oncotype DX (OncDx) is a 21-gene RT-PCR assay that estimates the likelihood of recurrence of estrogen receptor positive, HER2-negative breast carcinoma $(\mathrm{BC})^{1}$. The OncDx recurrence score (RS) is used in the treatment planning of patients (pts) with $\mathrm{BC}$, and pts with low RS (defined as RS $<18$ ) are usually spared chemotherapy ${ }^{1}$. The prognostic power of RS was reported to be independent of age using 50 years as cutoff point ${ }^{1}$. TailoRx is a prospective randomized trial to assess the benefit of chemotherapy for $\mathrm{BC}$ with $\mathrm{RS}$ 11-25. Initial results ${ }^{2}$ confirm low risk for $\mathrm{BC}$ with $\mathrm{RS} \leq 10$, but do not provide data for $\mathrm{BC}$ with $\mathrm{RS} 11-25$. We reviewed the outcome of patients with $\mathrm{BC}$ and $\mathrm{RS}<18$ treated at our center.

Design: We identified BC patients treated at our center between 09/2008 and 03/2013 and reviewed clinicopathological characteristics, OncDx RS, treatment and outcome data. The Institutional Review Board approved the study.

Results: In the study period, BCs from 2904 pts underwent OncDx testing. A total of $1545(53 \%)$ BCs had low RS [RS 0-10: 537 (18\%), RS 11-17: 1008 (35\%)]. Seven pts with RS $<18$ (1 RS 0-10; 6 RS 11-17) developed distant metastases with median disease free interval of 25 months (Table 1). Their median age at BC diagnosis was 39 years, and $6 \mathrm{pts}$ were premenopausal. BC of the $7 \mathrm{pts}$ had median size of $2.1 \mathrm{~cm}$, and was multifocal in 4 cases; none was well-differentiated. One pt had micrometastatic carcinoma in one lymph node. Six pts received hormonal therapy ( $\# 5$ declined Tamoxifen). Two pts received adjuvant cyclofosphamide, metotrexate and 5-fluorouracil (CMF).

\begin{tabular}{|l|l|l|l|l|l|}
\hline Case & RS & Age & BC size $(\mathrm{cm})$ & Time to metastasis $(\mathrm{mo})$ & Site of Mets \\
\hline 1 & 5 & 50 & $2.1^{\mathrm{a}}$ & 58 & Bone \\
\hline 2 & 12 & 54 & $1.3^{\mathrm{a}}$ & 41 & Multiple \\
\hline $3^{\mathrm{b}}$ & 12 & 37 & $2.7^{\mathrm{a}}$ & 25 & Lung \\
\hline 4 & 13 & 71 & $2.3^{\mathrm{a}}$ & 20 & Bone \\
\hline 5 & 14 & 38 & 1.6 & 48 & Bone \\
\hline $6^{\mathrm{b}, \mathrm{c}}$ & 16 & 39 & 1.2 & 6 & Bone \\
\hline 7 & 17 & 39 & 2.1 & 12 & Bone \\
\hline \multicolumn{5}{|l}{ a. Multifocal; b. Received adjuvant CMF; c. Node with micrometastasis } \\
\hline
\end{tabular}

Conclusions: Distant metastases developed in $0.5 \%$ of 1545 BC pts with OncDx $\mathrm{RS}<18$, mostly in the RS 11-17 group. Four of the 7 pts with distant metastases were younger than 40 years old at $\mathrm{BC}$ diagnosis, and 4 had multifocal disease. Our results indicate that in $\mathrm{BC}$ patients with $\mathrm{RS}<18$, multifocality and young age ( $<40$ years old) might have independent prognostic value.

References:

1. Paik S. NEJM 2004.

2. Sparano JA. NEJM 2015

\section{SAE2 Expression in c-MYC Positive Triple-Negative Breast} Carcinomas

Kimberly Lally, Xuemo (Sean) Fan, Serhan Alkan. Cedars-Sinai Medical Center, Los Angeles, CA.

Background: While triple-negative breast carcinoma (TNBC) represents approximately $10-20 \%$ of invasive breast cancers, its immunophenotypic profile has limited the availability of tailored therapy. Based on synthetic lethality mechanism, recent literature has reported that SUMO-activating enzyme subunit 2 (SAE2) may be a therapeutic target in breast carcinomas with MYC amplification; however the specific role of SAE2 in c-MYC-expressed TNBC requires additional investigation. Herein, a pilot study was undertaken to further characterize the expression of SAE2 in TNBC with MYC expression.

Design: Forty-five cases of patients with TNBC with c-MYC expression were selected from institutional archives. A representative formalin-fixed paraffin-embedded block was selected from each case and evaluated for both nuclear and cytoplasmic expression of SAE2 within tumor cells by immunohistochemistry (IHC).

Results: Samples included 45 TNBC cases, all of which expressed c-MYC. Forty-three of the total 45 cases $(95.5 \%)$ showed positive staining for SAE2, of which $9(21 \%)$ exhibited only nuclear staining, $0(0 \%)$ exhibited only cytoplasmic staining, and 34 $(79 \%)$ exhibited both nuclear and cytoplasmic staining. Among these cases, the intensity 
of staining ranged from $1+$ to $3+$ and the percentage of positive tumor cells ranged from $3 \%$ to $95 \%$. Several cases showed intratumoral heterogeneity with considerable variation in strength and pattern of staining within a given tumor.

Conclusions: Inhibition of SAE2, and subsequent alteration of sumoylation of various proteins, has been shown to impair MYC-driven tumorigenesis. Our study demonstrates that SAE2 is expressed in the majority of c-MYC-expressing TNBC cases. Given our previous studies have shown that MYC expression is commonly observed in TNBC, these results suggest that TNBC with MYC expression may be vulnerable to synthetic lethality by inactivation of SAE2. Thus, this finding warrants further clinical studies targeting SAE2 as a potential therapeutic option for MYC-driven TNBC.

\section{Androgen Receptor Expression in HER2-Positive Breast}

\section{Carcinomas}

Alexandra Larson, Irene B Helenowski, Jennifer Pincus, Julianne M Ubago, Adriana Rosca, Luis Z Blanco, Kalliopi P Siziopikou. Northwestern University Feinberg School of Medicine, Chicago, IL.

Background: Amplification of HER2 is found in $20-30 \%$ of breast cancers and confers a poorer prognosis. While treatment with trastuzumab has improved outcomes in this group, a subset of tumors develops resistance, requiring novel therapeutic options. Estrogen receptor (ER)+, HER2+ tumors (Luminal B) are another subtype of aggressive HER2+ breast carcinomas which could benefit from additional therapies. Interest in androgen receptor (AR) expression in breast carcinoma has increased as AR may represent a potential prognostic and predictive marker, especially for aggressive tumor subtypes and metastatic disease. In this study, we evaluated expression of AR in HER 2 + breast tumors.

Design: The study population consisted of 114 patients with HER2+ invasive breast carcinoma treated with surgical excision or mastectomy (mean age 53, range 18-80, period of study 2009-2014). Electronic medical records were reviewed for patient demographics. Pathologic tumor characteristics (histologic type, size, grade, lymph node status) and tumor marker profile (ER, PR, p53 \& Ki-67) were evaluated. Tissue microarrays were constructed (3 cores/case) for immunohistochemical evaluation of AR (Dako, AR441).

Results: Overall, AR was expressed in $78 \%$ of the HER2+ tumors (89/114). Expression of AR correlated with ER expression: $87 \%$ of Luminal B cancers expressed AR $(52 / 60)$ compared with only $69 \%$ in the HER2 subtype $(37 / 54)(\mathrm{p}=0.02)$. Dual ER/ AR expression was seen in 52/114 (45.6\%) of the cases. Of interest, AR+/ER+/HER2+ tumors were grade $2(36.5 \%)$ or grade $3(63.5 \%)$ tumors while AR-/ER-/HER $2+$ tumors were almost exclusively poorly differentiated, grade 3 tumors $(94.1 \%)(\mathrm{p}=0.01)$. No association of AR expression with age, race, tumor size, lymph node status, p53 expression or Ki-67 proliferation index was observed.

Conclusions: 1 . AR is expressed in over $3 / 4$ of the HER2+ carcinomas. 2. AR expression is more common in Luminal B compared to the HER2+ subtype of breast carcinomas. 3. HER2+ tumors with dual absence of AR \& ER are almost exclusively grade 3 carcinomas. 4. AR expression does not have a differential expression between age or racial groups and does not correlate with tumor size or lymph node status. Our findings add to the understanding of the molecular mechanisms that drive the different subtypes of breast carcinomas and raise the possibility of targeting the AR pathway as a novel therapeutic approach for the management of patients with HER $2+$ tumors.

204 Hyaline Fibrous Involution: A Novel Histologic Finding That Is More Common in BRCA+ Breast Tissue

Hee Eun Lee, Muhammad Arshad, Rushin D Brahmbhat, Tanya L Hoskin, Stacey Winham, Marlene H Frost, Derek C Radisky, Lori Denison, Amy Degnim, Daniel W Visscher. Mayo Clinic, Rochester, MN.

Background: We describe a possibly novel benign histologic entity in breast tissue and evaluate its frequency in benign breast tissues from women with and without known BRCA mutations. We term this finding "hyaline fibrous involution" (HFI), characterized by symmetric and regular deposition of basal lamina like periacinar hyaline material in association with atrophic epithelium.

Design: A cohort of 93 women with germline BRCA mutation who underwent prophylactic mastectomy (BRCA group) was compared to an age-matched sample of women who underwent biopsy for benign breast disease (BBD group). Median age was 45 years (range, 25-72 years). Representative sections were reviewed to evaluate the total number of terminal duct lobular units (TDLUs) and the number of TDLU with HFI in each case. The presence of any HFI and the prevalence of HFI lobules as a percent of total lobules were estimated and compared between groups. Logistic regression adjusted for total lobules was used to compare groups with respect to the presence of any HFI lobule, while negative binomial models for count data were used to analyze the number of HFI lobules with the logarithm of total lobules as an offset. Results: The median total number of lobules per sample was 106 for BRCA and 62 for BBD. Presence of any HFI was significantly more frequent in the BRCA group compared to the BBD group, $47.3 \%$ versus $15.1 \%$ ( $p<0.0001$ ) adjusted for total lobules. Within the BRCA group, frequency of any HFI present was significantly higher in the perimenopausal age group (45-55 years: $62.9 \%)$ compared to other age groups $(<45$ years, $44.4 \%$; $>55$ years, $15.4 \%), p=0.05$ and $\mathrm{p}=0.02$, respectively. In the subset who did have HFI, the percent of HFI lobules relative to total lobules was median $3.3 \%$ in BRCA specimens $(n=44)$ and median $3.2 \%$ in BBD $(n=14), p=0.14$.

Conclusions: Increased presence of HFI in the setting of BRCA mutation suggests that it may represent a pathologic entity, possibly reflecting abnormal involution or a response to DNA damage.
205 Validation of a "Random Forests" Based Model Using Routine Pathologic Features to Predict OncoType Dx Risk Scores

Zach Lee, Hyunseok Kim, Tyler A Jensen, Chris B Umbricht, Leslie Cope, Bradley Turner, David G Hicks, Martin Rosman, W Charles Mylander, Antonio C Wolff, Dylan Miller. University of Utah, Salt Lake City, UT; Hopkins University School of Medicine, Baltimore, MD; Johns Hopkins, Baltimore, MD; University of Rochester, Rochester, NY; Anne Arundel Medical Center, Annapolis, MD; Intermountain Medical Center $/$ University of Utah, Salt Lake City, UT; Intermountain Biorepository, Salt Lake City, UT. Background: Oncotype DX (ODX) is a gene expression profiling assay for breast cancer that yields a numeric recurrence risk score (RS). 21 genes comprise the ODX profile, but hormone receptor and proliferation genes are heavily weighted in the RS algorithm. Recent reports suggest that quantitative immunohistochemistry (IHC) for ER, PR, HER2, and Ki67 can closely approximate the ODX RS using linear regression models, at a fraction of the cost.

Design: Histopathologic and IHC results for ER, PR, HER2, and Ki67 were collected on cases with known ODX RS from 4 institutions (training set). A random forests algorithm was applied to the training set, identifying case clusters with similar grade and IHC patterns. A random forests-based model (RFM) (rather than linear regression) was developed to predict ODX RS high and low risk categories (ODX RS $>25=$ high risk) with $95 \%$ accuracy when a given case successfully matched a case cluster from the training set. This RFM was then applied to a validation set of new cases from our institution and compared to their actual ODX RS.

Results: Of 1,113 cases in the training set, $559(50 \%)$ showed enough cluster congruency by the RFM to be classified as low risk and $57(5 \%)$ could be classified as high risk. In 215 subsequent validation set cases, 111 (54\%) cases were classified as low risk by the RFM. $98 \%$ of these 111 were ODX RS low risk. $9(4 \%)$ were classified as high risk and all $(100 \%)$ had ODX RS high risk scores. For the remaining 95(46\%) patients in the validation set, the ODX RS could not be predicted with $95 \%$ accuracy.

Conclusions: This study further supports the premise that routine pathologic features can be used to approximate the ODX RS in a substantial proportion of cases, suggesting that an integrated RFM - ODX predictive testing model could be a feasible approach.
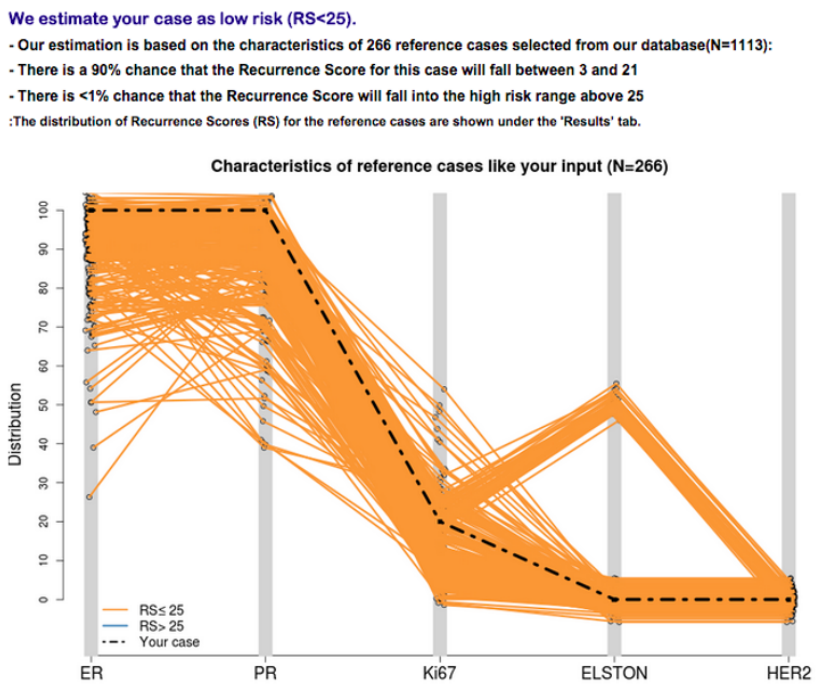

206 Intratumoral Heterogeneity of Common Protein Biomarkers Assessed by Immunohistochemistry in Breast Cancer Tissues

Jie Li, Theresa Dawidek, Veronika Liegsalz, Vasilis Tzalis, Anette Feuchtinger, Stefanie Avril. Case Western Reserve University and University Hospitals Case Medical Center, Cleveland, OH; Technische Universität München, Munich, Germany; Helmholtz Zentrum München, Neuherberg, Germany.

Background: Although protein biomarkers assessed by immunohistochemistry are commonly used as prognostic and predictive factors, variability of protein expression within a single tumor is not well studied. This study assessed potential intratumoral heterogeneity of protein expression a) within primary breast carcinomas and b) between axillary lymph node metastases from the same patient.

Design: The expression of 7 biomarkers including estrogen- (ER) and progesterone receptor (PR), HER2, Bcl-2, E-Cadherin, Ki-67 and cyclin-D1 was assessed by immunohistochemistry on 133 formalin-fixed and paraffin embedded tissue samples from 25 large $(\geq 3 \mathrm{~cm})$ primary invasive breast cancers. From each case 6-10 samples from different zones of the primary tumor (peripheral, intermediate, central) were analysed; in addition, 2-5 axillary lymph node metastases from 9 cases were included. Protein expression was quantified within 2-4 manually defined regions of interest (ROI) for each sample using Definiens image software. The percentage of positive tumor cells per number of tumor cells or tumor area was calculated for all markers, except for HER2 which was scored semiquantitative ( $1+$ to $3+$ ) according to ASCO/CAP guidelines. Results: All 7 proteins showed considerable intratumoral heterogeneity in their expression within primary breast cancers with a mean coefficient of variation $(\mathrm{CV})$ of $58 \%$ (range $42-90 \%$ ). The extent of intratumoral heterogeneity was different among the 7 individual proteins analysed, with Bcl-2 and E-Cadherin demonstrating the highest intratumoral variability. Interestingly, the extent of intratumoral heterogeneity within a defined tumor zone (mean CV $72 \%$, range $35-135 \%$ ) was not considerably lower than between different tumor zones (mean CV 46\%, range 31-70\%). Individual lymph node 
metastases from the same patient showed a similar heterogeneity in protein expression (CV 59\%, range 23-89\%). In comparison, we assessed the variation of protein expression amongst tumors from different patients, which revealed a CV of $72 \%$ (range 33-149\%) for primary tumor samples and $80 \%$ (range $18-145 \%$ ) for lymph node metastases. Conclusions: Our data indicate that assessment of established and novel proteins as diagnostic or prognostic markers may require sampling of the tumor in several distinct locations or sampling of several tumor-involved lymph nodes to avoid sampling bias.

207 Biomarkers Predicting Pathological Complete Response to Neoadjuvant Chemotherapy in Breast Cancer

Xiaoxian (Bill) Li, Uma Krishnamurti, Shristi Bhattarai, Sergey Klimov, Michelle Reid, Ritu Aneja. Emory University, Atlanta, GA; Georgia State University, Atlanta, GA. Background: Recent studies have shown strong correlation of pathological complete response (pCR) to neoadjuvant chemotherapy with survival and prognosis in breast cancers. The objective of this study is to correlate pCR with tumor morphology and biomarker status in order to determine which patients are most likely to benefit from neoadjuvant chemotherapy.

Design: Clinical data from 237 breast cancer patients who received neoadjuvant chemotherapy between 2012 and 2014 were reviewed. Correlations were sought between $\mathrm{pCR}$ and estrogen receptor (ER), progesterone receptor (PR), and HER2 status; Nottingham and nuclear grades; tumor tubule formation; mitotic score; Ki67 index; and tumoral and stromal lymphocytic infiltration (TLI and SLI, respectively). Results: Of the 237 cases, 104 (43.9\%) achieved pCR. The HER2-positive (HER2+) and triple negative breast cancer (TNBC) subtypes had higher pCR rates compared with the luminal subtype (ER or PR positive and HER2 negative). ER and PR negativity, HER2 positivity, Nottingham grade 3, increased TLI and SLI, high mitotic count and Ki67 score correlated significantly with pCR in the overall cohort. TLI and SLI correlated significantly with pCR in the HER2+ and TNBC subtypes in multivariate analysis, whereas no biomarkers correlated with pCR in the luminal subtype.

Conclusions: In addition to the pathologic parameters and biomarkers already routinely assessed, evaluation of TLI and SLI may help to better select patients with HER2positive and TNBC for neoadjuvant chemotherapy.

208 Management of Benign Papilloma of the Breast Diagnosed on Core Biopsy, a Review of $\mathbf{4 1 8 2}$ Cases

Xiaoxian (Bill) Li. Emory University, Atlanta, GA.

Background: Benign papilloma is a common diagnosis in the current era with wide usage of mammography screening. Whether to excise all benign papilloma diagnosed on core biopsy is controversial.

Design: Fifty three publications from 2000 to 2015 were fully reviewed. A total of 4182 cases of benign papilloma diagnosed on core biopsy were retrieved from the 53 studies. The upgrade rate to invasive carcinoma or ductal carcinoma in situ in subsequent excision or follow up, and radiological and pathological features associated with upgrade rate were examined.

Results: The upgrade rate ranged from $0-20 \%$ in the 53 studies. Overall, $205(4.9 \%)$ of the 4182 cases were upgraded. Large size of the lesion, palpable or mass forming, associated calcification, poor sampling by small gauge needles, pathology-radiology discordance, and uncertainty of pathological diagnosis were associated with upgrade rate.

Conclusions: When an accurate pathological diagnosis can be rendered, microscopic papilloma with concordant benign radiological findings can be safely followed. Small papilloma with mass formation or associated calcifications maybe followed if the lesion is well sampled and the pathological diagnosis is concordant with radiological findings. Multidisciplinary discussion among pathologists, radiologists and surgeons is essential for appropriate management.

\section{Male Breast Carcinoma Has Worse Prognosis Than Female Breast}

\section{Carcinoma}

Xiaoxian (Bill) Li, Uma Krishnamurti, Lei Huo, Limin Peng. Emory University, Atlanta, GA; University of Texas MD Anderson Cancer Center, Houston, TX.

Background: Male breast carcinoma (MBC) is uncommon. Compared with female breast carcinoma (FBC), the prognosis and clinico-pathological features of $\mathrm{MBC}$ have not been well studied.

Design: A total of 182,523 breast carcinoma cases were retrieved from the National Cancer Institute Surveillance, Epidemiology and End Results (SEER) database from 2010 to 2012. Carcinomas were classified into three subtypes: hormonal receptor positive $(\mathrm{HR}+)$ /HER2-, HER2+ regardless HR status and HR-/HER2-. Incidence, carcinoma subtype, stage and overall survival were examined.

Results: The overall incidence of MBC was $0.8 \%$ (1527 cases). Compared with FBC, MBC was more frequently HR+/HER2- (86\% vs $73 \%)$ and less frequently HR-/ HER 2 - ( $2 \%$ vs $12 \%, \mathrm{P}<.001)$. More MBC was staged as III or IV $(25 \%$ vs $17 \% \mathrm{FBC}$, $\mathrm{P}<.001)$. MBC had significant worse overall survival than $\mathrm{FBC}(\mathrm{P}<.0001)$. Stage I and II MBC patients had significantly worse survival than stage-matched FBC patients in $(\mathrm{P}<.001$ for stage $\mathrm{I}, \mathrm{P}<.001$ for stage II), which was not observed in stage III or IV patients. MBC patients had worse survival than $\mathrm{FBC}$ patients in all subtypes: $\mathrm{HR}+/$ HER2- $(\mathrm{P}<.001)$; HER 2+ $(\mathrm{P}<.0001)$ and HR-/HER2- $(\mathrm{P}=0.002)$. When comparison was made for stage-matched and subtype-matched patients, MBC patients had significantly or marginally significantly worse survival than FBC for stage I HR+/HER2- $(\mathrm{p}=0.001)$, stage I HR-/HER2- $(\mathrm{p}=0.048)$, stage II HR+/HER2- $(\mathrm{p}=0.017)$, stage II HER2+ $(p=0.044)$, stage III HER $2+(p<0.001)$, stage IV HR-/HER2- $(p=0.059)$. Multivariate analysis that adjusted for stage and subtype also indicated the survival disadvantage of MBC (Hazard Ratio=1.41, $\mathrm{p}=0.001$ ).
Conclusions: $\mathrm{MBC}$ has poorer survival than FBC. More studies are needed to elucidate the underlying mechanisms in order to optimize treatment options.

210 HER2 Gene Protein Assay Is Useful to Determine HER2 Status of Breast Carcinoma with HER2 IHC and FISH Double Equivocal Results

Zaibo Li, Yanjun Hou, Hiroaki Nitta. Ohio State University, Columbus, OH; Ventana Medical Systems, Inc, Tucson, AZ.

Background: HER2 status determined by HER2 assays is both a prognostic factor in breast cancer and a predictive factor for the effects of HER2-targeted drugs. Approximately $15 \%$ of breast cancers show equivocal HER2 results on HER2 immunohistochemistry (IHC) and are reflexed for fluorescence in situ hybridization (FISH). However, some of those cases remain equivocal after FISH. In this study, we evaluated these double equivocal cases by using a novel gene protein assay (GPA), which can simultaneously assess HER 2 gene copy number and protein on a single slide using bright-field microscopy.

Design: 42 HER2 IHC and FISH (CEP17 probe) double equivocal cases were retrieved from our pathology archive. Some of these cases also had repeated HER2 FISH with CEP17 probe or alternative probes (D17Z1 and D17S122) on either same or different tissue blocks. GPA was performed on these cases according to manufacturer's protocol. The GPA slides were interpreted according to the 2013 HER2 ASCO/CAP guidelines and the results were compared with FISH results.

Results: In 42 HER2 IHC and FISH double equivocal cases, GPA was negative for amplification/overexpression in 28 cases, equivocal in 3 cases and positive in 11 cases. For 28 GPA negative cases, 12 had repeat FISH (CEP17) with 10 as negative and 2 as equivocal. Both cases with equivocal repeat FISH (CEP17) and negative GPA results were negative by FISH with alternative probes. For 11 GPA positive cases, 8 cases had repeat FISH (CEP17) with 3 as positive and 5 as equivocal. FISH with alternative probes was performed in 3 cases with equivocal repeat FISH (CEP17) and positive GPA results and was positive in all 3 cases. For 3 GPA equivocal cases, 2 cases had repeat FISH (CEP17) and both cases were negative.

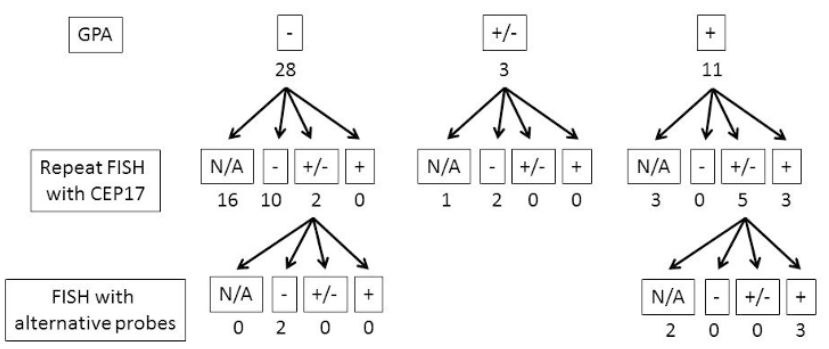

Conclusions: HER2 GPA performs accurately and is very useful to determine HER2 status in HER2 IHC and FISH double equivocal breast cancer cases.

\section{Genomic Profiling of Ductal Carcinoma In Situ: Can We Predict} Outcome?

Chieh-Yu Lin, Sujay Vennam, Robert T Sweeney, Shirley X Zhu, Sushama Varma, Robert $B$ West. Stanford University Medical Center, Stanford, CA.

Background: The detection rate of ductal carcinoma in situ (DCIS) of the breast has increased significantly, through advanced screening programs, without reducing breast cancer mortality, raising the concern that we are over-treating DCIS. To tackle the issue, there is a clinical need to stratify patients with DCIS for the risk of developing invasive breast cancer (IBC). While the genomic landscape of IBC has been extensively studied, the somatic mutation profiles of DCIS are still under investigation. Our lab has identified copy number gain of several chromosomal regions in DCIS that are associated with progression to IBC. In the current study, we performed targeted exome-sequencing of DCIS cases with or without concurrent or subsequent IBC. We aim to identify key somatic mutations that would allow us to calculate the risk of DCIS progression. Design: Targeted exome-sequencing with a custom panel of 223 targets was performed on 80 DCIS samples from archival blocks. 44 samples were from cases that have concurrent or subsequent IBC (DCIS+IBC group), and 36 samples were from cases that have no development of IBC over a median follow-up of 9 years (DCIS-only group). Somatic variants were analyzed using an ensemble approach combining three commonly used variant-analysis programs, Mutect, Freebayes and Vardic.

Results: There is no statistic significance between the two groups for ER status, PR status, and histological grade. Targeted exome-seqencing of the DCIS reveals an average of 5 somatic mutations per case in both groups. The majority of the variants are nonsynomonus mutations $(79 \%)$, while the rest include frame-shift, splicing, and stopgain mutations. Several known recurrent somatic mutations that have been identified in The Cancer Genome Atlas database of IBC are also detected, accounting for $13.8 \%$ of all the variant calls. A statistically significance enrichment of somatic mutations in the PI3K binding pathway is identified in the DCIS + IBC group.

Conclusions: Our study provides molecular clues to stratify risk of DCIS progression using targeted exome-sequencing. We identified recurrent somatic mutations in PI3K binding pathway that might contribute to progression to IBC. These findings, combined with our previously published observation of copy number alteration gain, provide novel prognostic biomarkers to distinguish low-risk versus high-risk DCIS. 
212 RUNX3 Epigenetic Inactivation Is Associated with EstrogenReceptor Positive Breast Cancer

Hui Liu, Zhantao Yan, Kai Cao, Jaime Rodriguez-Canales, Yongping Wu. Xuzhou Medical College, Xuzhou, Jiangsu, China; MD Anderson Cancer Center, Houston, TX. Background: Breast cancer is associated with molecular alterations including dysregulation of tumor suppressor genes via epigenetic changes. Runt-related transcription factor 3 (RUNX3), encoded by RUNX3 gene, was first recognized as a tumor suppressor gene in gastric carcinoma. Previous studies have shown that $R U N X 3$ may also play a role as a tumor suppressor gene in breast cancer by reducing estrogen receptor (ER) activity. Gene promoter hypermethylation is a major mechanism in the inactivation of $R U N X 3$ with loss of RUNX3 expression, which may favor tumor progression. Our goal is to study $R U N X 3$ promoter methylation and its correlation with RUNX3 expression and the ER status in breast cancer.

Design: Two breast cancer cell lines (MCF7 \& SKBR3) and a normal mammary epithelial cell line (MCF10A) were analyzed for $R U N X 3$ promoter methylation by methylation-specific PCR (msPCR), mRNA expression by RT-PCR, and protein expression using Western blot and immunohistochemistry (IHC). Additionally, 113 human breast cancer specimens were analyzed for $R U N X 3$ promoter methylation and IHC expression of RUNX3 and ER. To further study the effect of a methylation inhibitor on RUNX3 status, migration and invasion assays were used to study MCF7 cells before and after treatment with 5-Aza-2'-deoxycytidine (5-Aza-CdR).

Results: RUNX3 protein and mRNA were highly expressed in MCF10A cells but not in MCF7 and SKBR3 cells. The RUNX3 gene promoter showed hypermethylation in the MCF7 cells but not in the MCF10A and SKBR3 cells. In the tissue specimens, higher IHC expression of RUNX3 was seen in the ER-negative samples (67\%) than in the ER-positive samples $(27 \%)(P<0.01)$. Hypermethylation of the RUNX3 gene promoter occurred more frequently in ER-positive breast cancer tissues (82\%) than in ER-negative samples $(22 \%)(P<0.01)$. RUNX3 promoter hypermethylation correlated with tumor stage (in both ER-positive and ER-negative samples) and with lymph node metastasis (ER-positive samples) $(P<0.05)$. 5-Aza-CdR suppressed the proliferation, migration, and invasion of MCF7 cells, as well as reversed the hypermethylation status of $R U N X 3$ gene promoter and increased RUNX3 protein expression.

Conclusions: Our data strongly suggest a relationship between the methylation status of the RUNX3 gene promoter and ER-positive breast cancer. Hypermethylation of the $R U N X 3$ gene promoter may play an important mechanistic role in ER-positive breast carcinogenesis. Further studies on RUNX3 may provide novel insights in the treatment and prognosis of breast cancer and its potential value as a biomarker.

213 Analysis of Malignant Phyllodes Tumor (MPT) Genomic Landscape Using Capture-Based Next Generation Sequencing (Cb-NGS)

Su-Yang Liu, Nancy Joseph, Yunn-Yi Chen, Poonam Vohra, Gregor Krings. UCSF, San Francisco, CA

Background: MPT are rare aggressive tumors with risk for recurrence and metastasis, which has poor prognosis. Malignant heterologous differentiation (HD) is diagnostic of MPT and associated with metastasis, with liposarcomatous differentiation (LD) being most common. Identifying the molecular alterations of MPT may help elucidate the pathogenesis of these poorly understood tumors, but the genomic landscape remains ill-defined, and mechanisms of progression to HD are unknown. In this study, we comprehensively analyzed the genomic landscape of MPT, including MPT with LD, using Cb-NGS and compared areas with and without LD to better understand the pathogenesis, intratumoral heterogeneity and progression of these tumors.

Design: DNA was extracted from formalin-fixed paraffin embedded tissue from 7 MPT and matched normal tissue, including 5 MPT with LD. For the 5 cases of MPT with LD, classic spindle (CS) and LD areas were extracted separately. Cb-NGS was performed targeting coding regions of over 500 cancer genes and select introns, covering 2.8 Megabases. Duplicate sequence reads were removed computationally for accurate allele frequency determination and copy number calling. Single nucleotide variants(SNV), insertions/deletions(indels), and copy number alterations(CNA) were evaluated. Results: Recurrently mutated genes included TERT promoter (TERTp; 4/7), MED12 (2/7), FGFR1 (2/7), SETD2 (2/7) and TP53 (2/7). TP53 inactivation was biallelic in both cases. FGFR1 mutations were in the protein kinase domain. Two MPT had FGFR1, TERTp and MED12 mutations. Unique mutations included PIK3CA, ESR1, RB1, IGFR1, PDGFRB, RARA, VHL, CHEK2, NF1, among others. In MPT with LD, identical SNV/indels were present in LD and CS areas. Recurrent CNA included gains of chromosome(chr) 5 (3/7), 8q (4/7), 17q (4/7), 20 (4/7), and 21q (3/7) and losses of chr $16 q(3 / 7)$ and $22(3 / 7)$. In all MPT with LD with separately evaluable CS and LD areas $(n=4)$, additional CNA were present in CS areas compared to LD areas; 1 MPT with LD also showed unique CNA (9p, 6q and 11p loss) in the LD compared to CS area. MDM2/CDK4 amplifications were not present. No recurrent CNA specific to CS or LD were identified.

Conclusions: MPT show significant intertumoral and intratumoral heterogeneity. Mutations in TERTp, MED12, FGFR1, SETD2 and TP53 are recurrent and may be drivers. CNA highlight intratumoral heterogeneity in MPT with LD and suggest that tumor progression occurs predominantly in CS not LD areas. No differences in SNV or indels were found between CS and LD areas. MPT with LD do not harbor MDM2/ CDK4 amplifications typical of liposarcoma.
214 Analysis of MED12 and TERT Promoter Mutations in Fibroepithelial Lesions (FEL), Metaplastic Carcinomas (MC) and Spindle Cell Lesions (SCL) of the Breast

Su-Yang Liu, Yunn-Yi Chen, Nancy Joseph, Poonam Vohra, Gregor Krings. UCSF, San Francisco, CA.

Background: Phyllodes tumor (PT) and MC are rare tumors that may share histologic features due to stromal overgrowth or heterologous differentiation (HD) of PT. Diagnosis and distinction from other SCL may be challenging, especially on core biopsy (CB) but has clinical implications. MED12 mutations (MED12m) have been identified in FEL including PT, and a recent study found TERT promoter mutations (TERTpm) in PT, but neither have been evaluated in histologic mimics. In this study, we analyzed MED12m and TERTpm in FEL, MC and SCL to elucidate pathogenesis and determine utility in differential diagnosis.

Design: MED12 (exon 2) and TERTp hotspots were evaluated by Sanger sequencing in PT (benign [BPT], borderline [BLPT], malignant [MPT]) and compared to other FEL (fibroadenomas [FA] including cellular [CFA] and juvenile [JFA], periductal stromal tumors [PST]), MC (including spindle cell carcinomas [SCC] and MC with HD), and other SCL (fibromatosis [FM], myofibroblastoma [MB], nodular fasciitis [NF], PASH, gynecomastia [GM] and dermatofibrosarcoma [DFSP]).

Results: MED $12 \mathrm{~m}$ were identified in $44 \%$ of FEL including $47 \%$ of PT and were more common in BPT $(71 \%)$ than BLPT $(58 \%)$ or MPT $(23 \%)$. Of 3 MPT with MED12m, 1 had osteoclastic giant cells (OGC) and 2 had liposarcomatous HD, with MED12m in both HD and non-HD areas. No MC, PST or SCL had MED12m. Of FEL, TERTpm were present only in BLPT $(25 \%)$ and MPT $(50 \%)$. All MPT with MED12m had TERTpm; 3 MPT had TERTpm but not MED12m. TERTpm were found in $2(11 \%)$ $\mathrm{MC}$, one of which had OGC as well as TERTpm in ductal carcinoma in situ. No other SCL had TERTpm.

\begin{tabular}{|l|l|l|}
\hline & MED12m \%(n) & TERTpm \%(n) \\
\hline MC & $0(20)$ & $11(19)$ \\
\hline SCC + MC with HD & $0(15)$ & $7(14)$ \\
\hline PT & $47(32)$ & $30(23)$ \\
\hline BPT & $71(7)$ & $0(5)$ \\
\hline BLPT & $58(12)$ & $25(8)$ \\
\hline MPT & $23(13)$ & $50(10)$ \\
\hline FA & $33(9)$ & $0(10)$ \\
\hline CFA, JFA & $40(5), 25(4)$ & \\
\hline PST & $0(2)$ & $0(2)$ \\
\hline FM & $0(6)$ & $0(3)$ \\
\hline MB & $0(4)$ & $0(4)$ \\
\hline NF & $0(2)$ & $0(2)$ \\
\hline PASH & $0(4)$ & $0(1)$ \\
\hline GM & $0(3)$ & $0(3)$ \\
\hline DFSP & $0(4)$ & $0(1)$ \\
\hline p(PT v MC) & $<.01$ & .15 \\
\hline p(MPT v MC) & .05 & .03 \\
\hline p(MPT v SCC + MC with HD) & .09 & .05 \\
\hline p(MPT/BLPT v MC) & .001 & .06 \\
\hline p(MPT/BLPT v SCC + MC with HD) & .006 & .04 \\
\hline p(FEL v other) & $<.01$ & .08 \\
\hline
\end{tabular}

Conclusions: MED12m are specific for FEL and more common in BPT than BLPT or MPT. MED12m favor MPT/BLPT over MC or other SCL, which may be useful on CB. TERTpm are common in MPT and BLPT but not present in BPT, FA or SCL. TERTpm may favor MPT or BLPT over MC but are not specific, as rare MC also harbor TERTpm.

215 Towards Automated Histopathology of Breast Cancer Using Spatial Light Interference Microscopy (SLIM)

Hassaan Majeed, Tan Nguyen, Virgilia Macias, Krishnarao Tangella, Andre KajdacsyBalla, Minh Do, Gabriel Popescu. University of Illinois at Urbana Champaign, Urbana, IL; University of Illinois at Chicago, Chicago, IL.

Background: The two key limitations of the standard histopathology procedure for breast cancers are observer subjectivity and low throughput. These limitations arise because biopsy analysis requires manual microscopic examination by a pathologist. As a first step towards overcoming these limitations, we showed in our previous work that quantitative phase images of breast cancer tissue, obtained using SLIM, have sufficient resolution and contrast to allow tissue evaluation. In this work we highlight an automatic image segmentation method that delineates different tissue areas (stromal, epithelial etc.) in SLIM images of breast biopsies. This is a crucial next step in our search for a more viable histopathology method because different types of tissues need to be separated in images before parameters, that are indicative of tissue health, can be computed Design: A tissue microarray of 900 cores was imaged using our SLIM slide scanning module. Images of 50 cores were manually segmented and labelled into stromal, epithelial and lumenal areas and these data were used as a training set for our classifier. The classifier computed a response vector for each pixel using the LeungMalik (LM) filter bank and these response vectors were clustered using the K-means algorithm to obtain centers (called textons). The random forest algorithm was used to find a relationship between the pixel label and the histogram of the textons in its neighborhood. In the validation step, the classifier generated the texton histogram in 
the neighborhood of each pixel for the remaining 850 cores and generated a label for the pixel based on the model learnt in the training step, there by identifying the type of tissue the pixel belonged to.

Results: Figure 1. shows the segmentation results for one core in the TMA

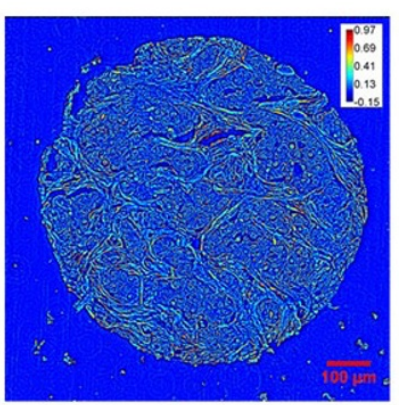

(a)

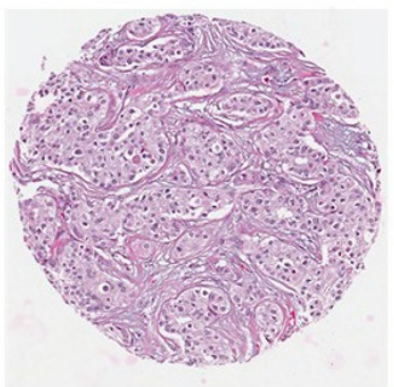

(b)

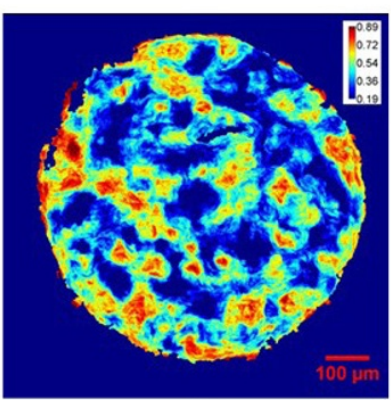

(c)

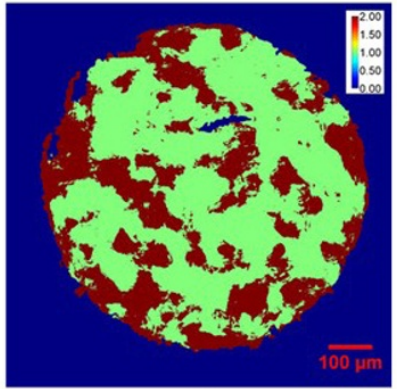

(d)
Figure 1. Segmentation results on a Breast TMA core (a) SLIM image (colorbar in radians) (b) H\&E stained tissue image of a corresponding core from a parallel tissue section (c) Probability map stained tissue image of a corresponding core from a parallel tissue section (c) Probability map
showing probability of a pixel being stromal (d) Segmented tissue image with lumenal pixels $=0$, glandular pixels $=1$ and stromal pixels $=2$.

Conclusions: Our classification method has the ability to automatically segment a SLIM image of breast tissue into different tissue components. Since the pixel values in SLIM images quantify physical properties of tissue, our classifier can potentially be used for histopathological analysis without the need for calibration leading to a high throughput and objective method for breast cancer evaluation.

\section{Breast Core Biopsy of Vascular Neoplasms -Pathologic Features,} Imaging and Clinical Findings

Jose Mantilla Arango, Esperanza Villanueva-Siles, Susan A Fineberg. Montefiore Medical Center, Bronx, NY.

Background: Accurate classification of vascular neoplasms of the breast can present significant challenges, especially on breast core biopsies (bcbx). Large series documenting clinico-pathologic and imaging findings of these lesions are few. In this series we review our 25 year experience.

Design: Using a data extraction engine, we identified $27 \mathrm{pts}$ with diagnosis of vascular lesion on bcbx from 1990 to 2015, for whom bcbx slides were available for review. Clinical and imaging findings were reviewed, as well as slides from 16 pts who had excisions.

Results: 3 out of 27 patients, (11\%) were male. The median age was 60 yrs (range $27-80$ ). Five pts ( $18 \%$ ) were Asian ( $\approx 1 \%$ of our population). Four pts had synchronous carcinoma, but no pt had a history of radiation. Presentation included palpable mass in 8 pts (30\% - including all 3 males), mammographic abnormality in 11 (41\%), MRI finding in one (4\%) and an incidental finding in $7(26 \%)$. Imaging impression was hematoma in 5 pts $(19 \%)$, lymph node in $2(8 \%)$, cyst in $2(8 \%)$ and fat necrosis in one $(4 \%)$. Median size was $7.5 \mathrm{~mm}$ (range 1.6-16mm). The lesions were classified, based on bcbx alone (11 pts) or bcbx and excision (16 pts) as: $1(4 \%)$ low grade angiosarcoma, $12(44 \%)$ hemangiomas, 9 (33\%) angiolipomas, $5(19 \%)$ perilobular hemangiomas (all incidental on bcbx). The angiosarcoma presented as a palpable mass which enhanced on MRI; on bcbx it had a size of $9 \mathrm{~mm}$, infiltrative margins, dissection of glands and stroma, anastomosing vascular channels, moderate cytologic atypia, high cellularity, no mitotic activity, Ki67 of 10\%, and no endothelial proliferative foci. Bcbx of 26 benign lesions showed cytologic atypia in 14 (mild in 8, moderate in 6); mitotic activity in 1, and circumscription in 12. In 6 cases, the lesions had infiltrative margins; in the remaining 8 cases, the lesion extended to the edges of the core, precluding full evaluation. Anastomosing vascular channels were seen in 10 lesions. Ki-67, done in 7 cases, was $<5 \%$ in 5 , and $\geq 10 \%$ in 2 . Dissection of glands and/or stroma was absent in benign lesions.

Conclusions: Low grade angiosarcoma and benign vascular neoplasms show overlapping features on bcbx, including infiltrative margins, cytologic atypia, mitotic activity, high Ki-67, anastomosing vascular channels, and possible overlap in size. The only difference was dissection of the normal glands and stroma, seen only in the angiosarcoma. Imaging and clinical findings are nonspecific. Most lesions will require excision, particularly when complete removal by imaging and definitive visualization of the entire lesion on biopsy cannot be confirmed.
217 The Genetic Landscape of Neuroendocrine Breast Carcinomas Caterina Marchio, Charlotte KY Ng, Salvatore Piscuoglio, Anne M Schultheis, Elena Guerini-Rocco, Marco Cupo, Felipe C Geyer, Anna Sapino, Britta Weigelt, Jorge $S$ Reis-Filho. University of Turin, Turin, Italy; Memorial Sloan Kettering Cancer Center, New York, NY; University Hospital, Cologne, Germany; European Institute of Oncology, Milan, Italy.

Background: Neuroendocrine breast carcinomas (NBCs) account for $2-5 \%$ of all invasive breast cancers and are histologically similar to neuroendocrine tumors from other sites. Contrary to other neuroendocrine cancers, NBCs typically express estrogen receptor (ER) and are of luminal subtype. The aims of this study were to define the mutational profile of NBCs, and to investigate whether NBCs and common forms of luminal (ER+/HER2-) breast cancer display distinct repertoires of somatic mutations. Design: Eighteen ER+/HER2- NBCs, defined as invasive breast carcinomas where $>50 \%$ of tumor cells expressed chromogranin and/or synaptophysin, and matched normal tissue were microdissected, and subjected to massively parallel sequencing targeting all exons of 254 genes most frequently mutated in breast cancer or related to DNA repair. Somatic point mutations were identified using MuTect; somatic insertions and deletions were defined using Strelka and Varscan2. The mutational repertoire of NBCs affecting these 254 genes was compared to that of ER+/HER2- breast cancers $(\mathrm{n}=241)$ and PAM50-defined luminal breast cancers $(\mathrm{n}=209$ Luminal A; $\mathrm{n}=112$ Luminal B) from The Cancer Genome Atlas (TCGA) project.

Results: NBCs were found to harbor a median of 4.5 (range 1-11) somatic mutations, which is similar to that of ER+/HER2- and Luminal B breast cancers but significantly higher than that of Luminal A carcinomas $(\mathrm{p}=0.04)$. The most frequently mutated genes in NBCs were GATA3, FOXA1, TBX3 and ARIDIA (all 3/18, 17\%), and PIK3CA and KIT (both $2 / 18,11 \%$ ). NBCs less frequently harbored PIK3CA mutations than common type ER+/HER2- breast cancers ( $11 \%$ vs $41 \%, \mathrm{p}=0.01)$, and displayed significantly higher frequency of somatic mutations affecting the chromatin remodeling gene ARIDIA $(17 \%$ vs $2 \%, \mathrm{p}=0.01)$ and the transcription factors FOXA1 and $T B X 3(17 \%$ vs $2 \%, \mathrm{p}=0.01)$. The repertoire of somatic mutations found in NBCs was more similar to that of Luminal B than that of Luminal A breast cancers, except for: i) TP53 somatic mutations, found in $29 \%$ of Luminal B cancers but in none of the NBCs ( $\mathrm{p}=0.003)$; and ii) ARIDIA and FOXA1 mutations, both detected in $17 \%$ of NBCs vs $3 \%$ and $1 \%$ in Luminal B cancers $(\mathrm{p}<0.05)$, respectively.

Conclusions: NBCs display a repertoire of somatic mutations similar to that of common forms of Luminal B carcinomas, but differ from those by the significantly lower frequency of TP53 mutations and a significantly higher prevalence of ARIDIA and FOXA1 mutations.

218 Is It Worth Performing ER, PR and HER2 Assays on All Concurrently Sampled Ipsilateral Breast Cancers; Study of 174 Biopsies from 85 Patients Shivali Marketkar, Kamaljeet Singh, Katrine Hansen, Margaret Steinhoff. Women and Infants Hospital, Providence, RI; Rhode Island Hospital, Providence, RI.

Background: The guidelines for ER, PR \& HER2 testing on concurrently sampled ipsilateral invasive breast cancers are not well defined. The CAP/ASCO guidelines recommend that testing should be performed on at least one of the tumors, preferably the largest. It is not clear if biomarker testing of all invasive tumors provides any clinically relevant information. We aimed to investigate the concordance rate of ER, PR \& HER2 results on concurrently sampled ipsilateral invasive breast tumors.

Design: Ipsilateral concurrently sampled breast tumors from 01/2010-07/2015 were identified. ER, PR \& HER2 immunohistochemistry(IHC) \& HER2 FISH expression was assessed using CAP/ASCO guidelines. Results were compared between the concurrently sampled tumors. Extent \& intensity of staining was noted in ER or PR discordant $(+\&-)$ tumors. Equivocal HER2 IHC was reflexed to FISH.

Results: Total 174 biopsies from 85 patients(pts) were identified and included 4 pts with 3 biopsies \& 81 pts with 2 biopsies. Histology was concordant in 81 pts: 69 ductal, 10 lobular \& 2 ductal \& lobular carcinoma, \& discordant in 4 pts: ductal vs. lobular $(\mathrm{n}=4)$. Histologic grade was different in $11(13 \%)$ pts. ER \& PR expression was different in $2(2.6 \%) \& 6(7.1 \%)$ cases respectively. Discordant ER tumors were PR- \& comprised of ER- \& low ER+(1-10\%; weak intensity) tumors. All six PR discordant tumors were ER+. HER2 IHC was concordant in 61(72\%) \& discordant in $24(28 \%)$ pts. FISH was concordant in 22/24 HER2 equivocal set of tumors. There were 23 HER2+ cases $(20 \mathrm{IHC}+\& 3 \mathrm{FISH}+)$, \& overall 4/85(4.7\%) pts showed discordant HER2(+\&-) tumors. Total $71 \mathrm{FISH}$ assays were done. Discordance in biomarker was independent of histologic grade. No discordance was identified in lobular tumors.

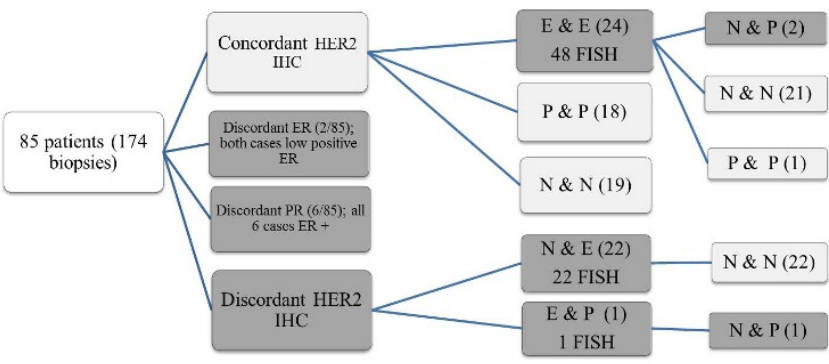

Conclusions: Concurrently biopsied ipsilateral breast tumors show a low discordance rate in biomarker expression. ER \& PR testing of all concurrently sampled tumors can be limited to ER/PR- \& low ER + tumors. With grade 1 or lobular tumors, HER2 testing of only one sample, preferably the higher-grade tumor is sufficient. HER2 testing should be done on all the separately sampled histologic grade $2 \& 3$ tumors. 
219 Intraoperative Consultation of Sentinel Lymph Nodes for Metastatic Breast Carcinoma in the Context of the ASCOSOG Z0011 Trial

Robert McDonald, Patrick C McGrath, Reed Spaulding, Joanna Dalland, Shannon Crook, Luis Samayoa. University of Kentucky, Lexington, KY.

Background: Axillary lymph node dissection (ALND) remains the standard of care for patients with a $>2 \mathrm{~mm}$ positive $(+)$ sentinel node(SN). Based on the efficacy of axillary ultrasound + - - fine needle aspiration (US $\pm \mathrm{FNA}$ ) in detecting $\geq \mathrm{N} 2$ disease and in the context of Z11, immediate ALND following a (+) intraoperative consultation (IOC) may prove unnecessary. This study focuses on the incidence and tumor burden of axillary disease in patients with a clinically and sonographically negative (-) axilla and their need for immediate ALND.

Design: We retrospectively reviewed 1162 patients $(2006-2015)$ with clinically and sonographically (-) axilla that underwent SN biopsy with or without IOC or ALND. Results were tabulated and compared in terms of type of surgery (lumpectomy vs mastectomy), number and status of SN and non-sentinel nodes (NSN), and final axillary stage.

Results: See Tables 1 and 2 and Figure 1.

Table 1 - Patients with SN from 2006-2015

\begin{tabular}{|l|l|l|l|l|l|}
\hline & $\begin{array}{l}\text { Total SN } \\
(-) \text { or } \\
\text { axilla (-) }\end{array}$ & $\begin{array}{l}\text { Total SN } \\
(+) \text { or } \\
\text { axilla }(+)\end{array}$ & $\begin{array}{l}\text { SN }(+) \\
\text { with } \\
\text { no ALND }\end{array}$ & $\begin{array}{l}\text { SN }+ \\
\text { NSN- }\end{array}$ & $\begin{array}{l}\text { SN+ } \\
\text { NSN+ }\end{array}$ \\
\hline Mastectomy with IOC & 509 & 132 & 17 & 78 & 37 \\
\hline Lumpectomy with IOC & 339 & 60 & 13 & 38 & 9 \\
\hline Lumpectomy without IOC & 93 & 8 & 5 & 3 & 0 \\
\hline SN failure & - & 21 & - & - & - \\
\hline Total cases & 941 & 221 & 35 & 119 & 46 \\
\hline
\end{tabular}

Table 2 - Axillary Stage of Patient Population

\begin{tabular}{|c|l|l|l|l|l|l|l|}
\hline & N0 & N0(ITC) & N(mic) & N1a & N2 & N3 & $(+)$ Axilla Total \\
\hline $\begin{array}{c}\text { \# patients } \\
(\mathrm{n}=1162)\end{array}$ & $\begin{array}{l}151 \\
(81 \%)\end{array}$ & $\begin{array}{l}15 \\
(1.3 \%)\end{array}$ & $\begin{array}{l}60 \\
(5.2 \%)\end{array}$ & $\begin{array}{l}116 \\
(10 \%)\end{array}$ & $\begin{array}{l}(2.2 \%) \\
(2.2 \%)\end{array}$ & $\begin{array}{l}(0.34 \%) \\
(19 \%)\end{array}$ \\
\hline
\end{tabular}

Figure 1 - Lymph Node Involvement in Patients with $\leq$ N1a Disease by SN and NSN Status

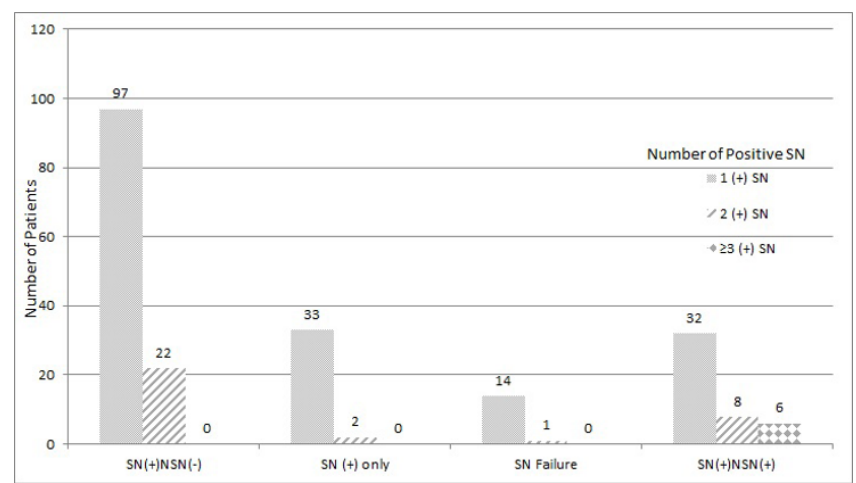

Conclusions: In patients with (-) US $\pm \mathrm{FNA}$, the incidence of $\geq \mathrm{N} 2$ disease $(2.6 \%)$ and number of $\mathrm{LN}$ with metastases were low. In this group, IOC could potentially increase the risk of comorbidities associated with an unnecessary ALND. In the context of Z11, the majority of patients with a clinically and radiographically (-) axilla may not need immediate ALND and do not require SN IOC.

220 Expression of Traditional Melanocytic Markers in Invasive Mammary Carcinoma

Stephanie McGregor, Gabrielle M Baker. University of Chicago Hospitals, Chicago, IL. Background: Immunohistochemistry (IHC) is a mainstay for identifying the primary site of metastatic disease, but even seemingly specific markers may demonstrate aberrant expression that can be difficult to interpret. S100 and SOX10 are regarded as robust markers of melanocytic differentiation but have also been demonstrated in invasive mammary carcinoma (IMC). We sought to evaluate the extent of co-expression of traditional markers of melanocytic differentiation in IMC subtypes.

Design: We constructed a tissue microarray consisting of 100 IMC cases: 7 ductal grade I (IDC-I), 8 IDC-II, 49 IDC-III, 14 metaplastic (MP), 6 IDC-III with focal MP features, 10 classic lobular/tubulolobular (ILC), and 6 pleomorphic ILC. Routine H\&E and IHC stains for S100, SOX10, MelanA, MITF, and HMB45 were performed. Adequately represented cases were scored for the percentage of positive nuclei and for cytoplasmic intensity.

Results: At least one marker was present in $82 \%$ of cases (S100: $62 \%$, HMB 45 : 36\%, SOX10: $32 \%$, MITF: $23 \%$, and MelanA: $9 \%$ ). While SOX10 and S100 were frequently present in $>50 \%$ of tumor nuclei ( $22 \%$ and $11 \%$ of cases, respectively), stains for MelanA, HMB45 and MITF were mostly weak and focal $(<10 \%$ of cells). Cytoplasmic S100 was present in $58 \%$ of cases ( $28 \% \geq$ diffuse weak/focal strong). At least 2 markers were seen in $54 \%$ of cases, with more markers on average in cases with MP features (43\% of cases with 3-4 markers, see figure). Of note, within MP cases, high co-expression of SOX10 and S100 was more common in cases with squamous or chondroid differentiation/matrix production than in those that were purely spindled.

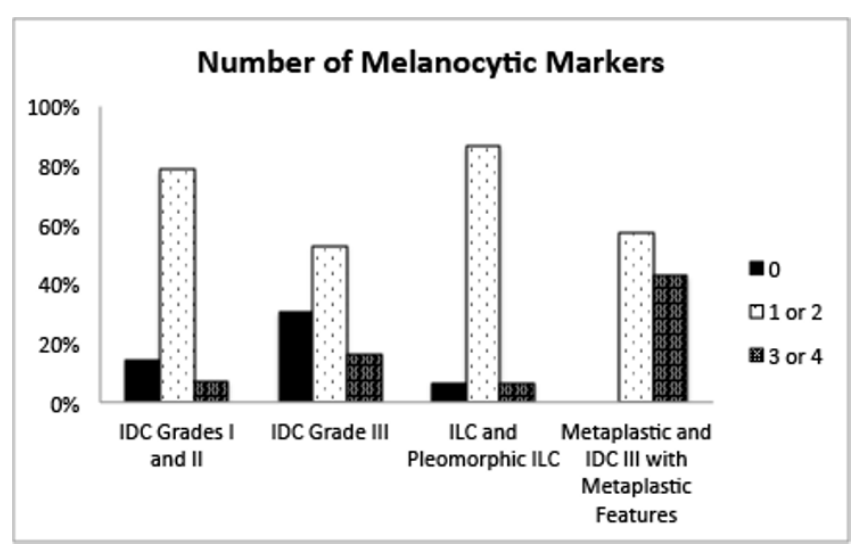

Conclusions: Multiple "melanocytic" markers can be co-expressed in IMC. SOX10 and S100 co-expression in particular can be extensive and therefore misleading if regarded as definitive evidence of melanocytic differentiation in a metastasis. In contrast, HMB45, MITF and MelanA tend to be diffuse in melanoma but focal and weak in IMC; however, it is important to note that these markers may be lost in a subset of melanoma. As SOX10 and S100 co-expression appears most common in higher grade tumors, particularly MP, this finding must be interpreted with caution in the setting of a possible breast primary and a panel approach of multiple markers may be necesary to ensure accurate diagnosis.

221 Reduced BAP1 Expression in Invasive Carcinoma of the Breast Stephanie McGregor, Gabrielle M Baker. University of Chicago Medicine, Chicago, IL. Background: BRCA-Associated Protein 1 (BAP1) is a deubiquitylase with classical tumor suppressor activity that is normally localized to the nucleus. Loss of BAP1 function is associated with various malignancies and is typically demonstrated by loss of nuclear staining using immunohistochemistry. Despite the well established role of BRCA $1 / 2$ in breast cancer and the functional cooperation of BAP 1 and BRCA proteins, loss of BAP1 in breast cancers has not been described.

Design: We constructed a tissue microarray consisting of 100 cases of invasive mammary carcinoma (IMC), including 7 ductal grade I (IDC-I), 8 IDC-II, 49 IDC-III, 14 metaplastic (MP), 6 IDC-III with focal MP features, 10 classic lobular/tubulolobular (ILC), and 6 pleomorphic ILC. Routine H\&E and immunohistochemistry for BAP1 was performed.

Results: Adequately represented cores were assessed for BAP1 staining. In addition to the expected nuclear staining, some degree of cytoplasmic staining was present across all cases with variable intensity in both components. A pattern emerged in which there were two apparent categories. The first ( $70 \%$ of cases) demonstrated clear nuclear expression of BAP1 that was easily discernible from the surrounding cytoplasm (left panel of accompanying figure). The second ( $30 \%$ of cases) encompassed a spectrum of findings that ranged from diminished intensity of BAP1 staining alone (middle panel) at one extreme to an apparent accumulation of granular cytoplasmic staining that obscured the subcellular localization of the BAP1 stain (right panel) at the other. The proportion of cases falling into the second category varied with histologic subtype as follows: ILC/ pleomorphic ILC - 13\%, IDC-I and II - 27\%, IDC-III - 35\%, and metaplastic/IDC-III with focal MP features $-37 \%$.

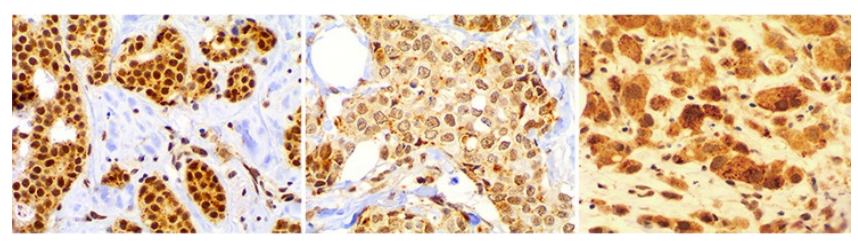

Conclusions: None of our examined cases demonstrated complete absence of nuclear BAP1 staining. However, there was an altered staining pattern in $30 \%$ of cases that consisted of a spectrum involving diminished nuclear BAP1 intensity and coarsely granular cytoplasmic staining that in some cases obscured the nuclear staining. This latter finding was more common in the higher grade ductal carcinomas, including cases with metaplastic features. These results suggest that diminished BAP1 function may play a role in the pathogenesis of invasive breast carcinoma.

222 Use of an Immunoprofile to Predict Response of Invasive Breast Cancer to Neoadjuvant Chemotherapy

Lauren E McLemore, Joseph Albanese, Yungtai Lo, Murali Janakiram, Susan A Fineberg. Montefiore Medical Center and the Albert Einstein College of Medicine, Bronx, NY; Albert Einstein College of Medicine, Bronx, NY.

Background: Response to neoadjuvant chemotherapy (NAC) in invasive breast cancer (IBC) is partly regulated by the immune microenvironment. We evaluated three components of the immune microenvironment, including $\mathrm{T}$ cell inhibitory molecule PDL-1, stromal tumor infiltrating lymphocytes (sTIL) and tumor associated macrophage (TAMs) and correlated with response to NAC in IBC.

Design: We identified 42 cases of IBC treated with NAC. We estimated \%sTIL in pre-chemotherapy core biopsies and used $>40 \%$ sTIL to define lymphocyte rich IBC. We performed immunohistochemistry for PDL-1 and macrophage marker CD68 on 
pre-chemotherapy breast core biopsies. PDL- 1 was positive if $\geq 1 \%$ of tumor cells or sTIL showed expression. CD68 was $3+$ positive if an average of $\geq 300+$ staining cells were identified in multiple hpf within the tumor bed. We used pathology reports from post NAC resections to calculate residual cancer burden (RCB) and considered RCB $0 / 1$ to represent excellent pathologic response and $\mathrm{RCB} 2 / 3$ to represent significant residual disease.

Results: Twenty of 42 cases $(47.6 \%)$ demonstrated excellent pathologic response to NAC (RCB 0/1) and $22(52.3 \%)$ demonstrated significant residual disease (RCB2/3). sTIL $\geq 40 \%$, ,CD68 $3+$ and +expression of PDL-1 were all associated with excellent pathologic response $(\mathrm{p}<.001)$ :

\begin{tabular}{|l|l|l|l|}
\hline & RCB0/1 & RCB2/3 & p value \\
\hline Stromal TIL $>=40 \%$ & 12 & 0 & $<.001$ \\
\hline Stromal TIL $<40 \%$ & 8 & 22 & \\
\hline & & & \\
\hline CD68 3+ positive & 10 & 0 & $<.001$ \\
\hline CD68 1-2+ & 10 & 22 & \\
\hline & & & \\
\hline PDL-1 positive & 17 & 6 & $<.001$ \\
\hline PDL-1 negative & 3 & 16 & \\
\hline
\end{tabular}

There was a association between sTIL $\geq 40 \%$ and CD68 $3+(\mathrm{p}<0.001)$ and PDL1+(p $=0.005$ ). Additionally, all 14 IBC demonstrating at least two of these three characteristics showed excellent pathologic response (RCB0/1). Sensitivity and specificity of using at least two of the three characteristics together to identify excellent pathologic response (RCB0/1) was 70\% (14/20) and 100\% (22/22), respectively. This combination was more sensitive than using sTILs alone to predict response (sensitivity $60 \%$ ).

Conclusions: In addition to sTIL, other components in the immune microenvironment can predict response to NAC. An immunoprofile including assessment of tumor associated macrophages and PDL1 expression in addition to sTIL was more sensitive and as specific as sTILs alone.

223 Proportion of Estrogen Receptor Positive and HER2 Positive Breast Cancer in a Rural Rwandan Cancer Center When Considering Duration of Ischemic and Fixation Time

Emily Meserve, Gaspard Muvugabigwi, Irenee Nshimiyimana, Krishan Taneja, Lydia Pace, Danny A Milner, Lawrence N Shulman, Deborah A Dillon, Tharcisse Mpunga. Brigham \& Women's Hospital, Boston, MA; Butaro Cancer Center of Excellence, Butaro, Rwanda; University of Pennsylvania, Philadelphia, PA.

Background: In sub-Saharan Africa (SSA), length of ischemia and formalin fixation may be difficult to control, jeopardizing reliability of immunohistochemical (IHC) markers necessary for breast cancer classification and treatment. We provide diagnostic pathology services to a rural Rwandan cancer center. Because variable proportions of estrogen receptor (ER) and HER2 positive breast cancer are reported in SSA, we evaluated the proportion of ER and HER2 positive tumors in our Rwandan patients with attention to cases with known length of ischemic and formalin fixation time.

Design: Cases included 331 female breast carcinomas received by our institutions from Jan 2012 to Dec 2014. The dates of biopsy/surgery, initial formalin exposure, and processing into paraffin blocks were obtained from clinical and laboratory records. Morphologic and IHC data were collected from pathology reports. Confirmatory HER2 FISH was performed on 12 cases.

Results: Average patient age was 51.8 years. Tumors showed ductal morphology ( $82 \%)$ and were often high grade (57\%). All 331 samples were placed in formalin within 24 hours; of these, $43.5 \%(144 / 331)$ were formalin fixed for $<72 \mathrm{hrs}$ before processing (average length 20.8 days; range 0-366). ER IHC was performed on $99 \%$ of cases, of which $64.3 \%$ were positive. HER2 IHC was performed on $51.9 \%$ of cases, of which $30.2 \%$ were $2+$ or $3+$ positive; with FISH confirmation, $24.7 \%$ were HER 2 positive. Of cases with $<72 \mathrm{hrs}$ formalin fixation, $63.89 \%$ (92/144) were ER positive and $28.3 \%$ (40/141) were HER2 $2+$ or $3+$ positive. Of 12 cases with equivocal IHC results, 4 showed amplification by FISH, resulting in HER2 positivity in $22.7 \%$ of cases. When comparing all cases to those with limited ischemic and formalin fixation time, neither the decrease in proportion of ER positive ( $64.3 \%$ to $63.9 \%)$ nor HER2 positive $(24.7 \%$ to $22.7 \%$ ) tumors were statistically significant ( $p>0.05)$.

Conclusions: Despite a long average length of formalin fixation, $43.5 \%$ of samples had $<24 \mathrm{hrs}$ ischemic time and $<72 \mathrm{hrs}$ formalin fixation. Compared to the US, our population demonstrates a lower proportion of ER positive tumors and a higher proportion of HER2 positive tumors, which were unaffected by duration of formalin fixation. These data emphasize the need for individual institutions in SSA to assess subtypes of breast cancer in their patient populations with attention to technical parameters to assure IHC marker reliability.

\section{A Longitudinal Study of LCIS on CNB}

Lavinia Middleton. MD Anderson, Houston, TX.

Background: In 2002 we reported a study of LCIS diagnosed on core needle biopsy and recommended that patients who presented with abnormal calcifications without a mass lesion or synchronus breast cancer should undergo observation rather than excision. The 2002 study examined over 2000 breast core needle biopsies obtained from 1995 to 2001 and identifed 35 women with atypical lobular hyperplasia or lobular carcinoma as the most significant lesion. Excisional biopsy was performed in 17 women and 18 patients were followed. This current study examines the long term follow up of these latter patients and reiterates recommendations for surveillence without surgical intervention in patients with incidental LCIS and ALH.
Design: The pathology database was searched and 2337 core needle biopsies from January 1, 1995 to December 31, 2001 were retrospectively reviewed for cases with the primary diagnosis of classic lobular carcinoma in situ, atypical lobular hyperplasia or lobular neoplasia. Thirty-five cases were identified. Lesions were re-reviewed and categorized based on published histological criteria. Clinical information was extracted from the patients' medical records. Radiographic findings were reviewed to determine the mammographic features of each lesion including whether architectural distortion or calcifications were present, size, and BI-RADS classification. Seventeen patients underwent excsional biopsy and the results were previously reported. Eighteen patients underwent surveillence and this study reports the long term outcomes of this latter group of patients after re-review of clinical and radiographic findings.

Results: One patient developed a multifocal invasive lobular carcinoma that measured $3 \mathrm{~cm}$ in largest single dimension 14 years after initial biopsy with negative annual interval mammograms. One additional patient had a stereotactic guided biopsy eight years after the initial biopsy which showed a radial scar and ALH and is currently without disease. The remaining patients had neither an abnormal mammogram nor biopsy with follow-up ranging from 6 to 14 years ( average 10.8).

Conclusions: This longitudal study reports over a decade of clinical experience with observing patients with lobular carcinoma in situ and atypical lobular hyperplasia diagnosed by core needle biopsy. We present over 10 years of follow-up for patients with incidental ALH and LCIS, identified by screening mamography and treated with chemoprevention. Disease progression was noted in one patient after 14 years $(5.5 \%)$ These results support earlier conclusions and provides compelling evidence to support clinical practice decisions to observe rather than excise incidental ALH and LCIS.

225 Subset Populations of Patients with Triple Negative Breast Cancer Exist Possessing Biomarkers for Targeted Therapeutics

Wilfrido Mojica, Paul Mojica, Elizabeth Marchetti. University at Buffalo, Buffalo, NY; UCLA, Los Angeles, CA.

Background: Plasma membrane proteins (PMP's) are ideal targets for precision medicine because their location may make them accessible to therapeutic intervention through cell-surface mediated interactions. PMP's have been traditionally difficult to characterize due to their amphiphilic nature. Recently, a tandem mass spectrometry study (Ziegler et al, PLoS One 2014;9(7):e102341) was successful in identifying several PMP's from cultured breast tumor cell lines, some of which were from triple negative breast cancers (TNBC). Because there is a paucity of targets for TNBC, we investigated these in vitro findings on clinical samples with the goal of validating the presence of new biomarkers on TNBC.

Design: PMP's with the highest spectral counts relative to normal breast cells were selected from the previous study. This list was narrowed down to four proteins based on their restricted expression on normal tissue (as identified on the Human Protein Atlas) and the paucity of previous reports in the scientific literature on these biomarkers in TNBC. Antibodies to L1CAM, CNTN1 (ThermoScientific), CDH2 (Life Technologies) and CDH19 (SigmaAldrich) were purchased and experimental conditions optimized using a R2000 autoclave for antigen retrieval with R-Universal Epitope Retrieval Buffer (Electron Microscopy Sciences) followed by incubation in an Antibody Amplifier overnight at $4^{\circ} \mathrm{C}$. Antibodies with optimized conditions were then tested on 72 cases of TNBC and 10 cases of non-neoplastic breast tissue (NNBT) previously identified from the department of pathology archival files.

Results: Optimal conditions could not be established for the antibodies to CDH19 and CNTN1. CDH2 demonstrated cytoplasmic staining in $7 \%$ and membranous staining in $17 \%$ of the TNBC cases. In the NNBT there was patchy membranous staining in $60 \%$ of the cases. L1CAM demonstrated distinct membranous staining in $42 \%$ of the TNBC and none of the epithelial cells in the NNBT cases. In the majority of cases where membranous staining was noted, different groups of tumor cells marked with either CDH2 or L1CAM, but not both.

Conclusions: The confirmation of $\mathrm{CDH} 2$ and L1CAM plasma membrane expression in a subset of clinical TNBC cases confirms the existence of potential biomarkers for development of either immunotherapy or antibody-drug conjugate based approaches in a tumor currently with limited treatment options. When these therapeutic options are developed and become available, immunohistochemistry will play a key role in guiding patient treatment.

226 Indeterminate Fibroepithelial Lesions on Breast Core-Needle Biopsy: Patient Characteristics, Management and Outcomes

Kelly Mooney, Kara-Lee Pool, David Y Lu, Peggy Sullivan. David Geffen School of Medicine at UCLA, Los Angeles, CA.

Background: Due to overlapping histologic and imaging characteristics, it can be difficult to distinguish fibroadenoma from phyllodes tumor on core-needle biopsy (CNB) of the breast. Management of CNB cases indeterminate for phyllodes tumor is controversial, ranging from observation to wide surgical excision. The aim of this study is to assess the clinical characteristics, management and outcome of patients with a diagnosis of an indeterminate fibroepithelial lesion (IFL) on CNB.

Design: A single institution, retrospective review was performed from January 1, 2004 to December 31, 2014. Cases with a diagnosis of IFL on breast CNB with phyllodes tumor reported in the differential were included. Patient age, size of mass, surgical excision result, recurrences and clinical follow-up based on last mammogram were recorded. Analysis was performed using unpaired two-tailed t-tests.

Results: 139 IFL CNB cases were analyzed. 100 (72\%) cases underwent excision, while $39(28 \%)$ cases did not. The average patient age and mass size on imaging was approximately the same for those who underwent excision (36 years old, mass size $2.1 \mathrm{~cm}$ ) compared to those who did not ( 37 years old, mass size $2.1 \mathrm{~cm}, \mathrm{p}=0.7$ ). Of the 100 cases that underwent excision, $81(81 \%)$ had a final diagnosis of fibroadenoma or benign non-fibroepithelial lesion and $19(19 \%)$ had a final diagnosis of phyllodes 
tumor (14 benign, 4 borderline, 1 malignant). Compared to patients without phyllodes tumor on excision, patients with phyllodes tumors were relatively older ( 42 vs 36 years, $\mathrm{p}<0.05)$, with larger mass sizes $(2.5 \mathrm{vs} 2.1 \mathrm{~cm}, \mathrm{p}=0.08)$. One patient with a borderline phyllodes tumor and one with a benign phyllodes tumor had recurrences 36 and 15 months after excision, respectively. No other recurrences or metastases were reported. All patients with available clinical follow-up were alive without disease (mean imaging follow-up 3 years).

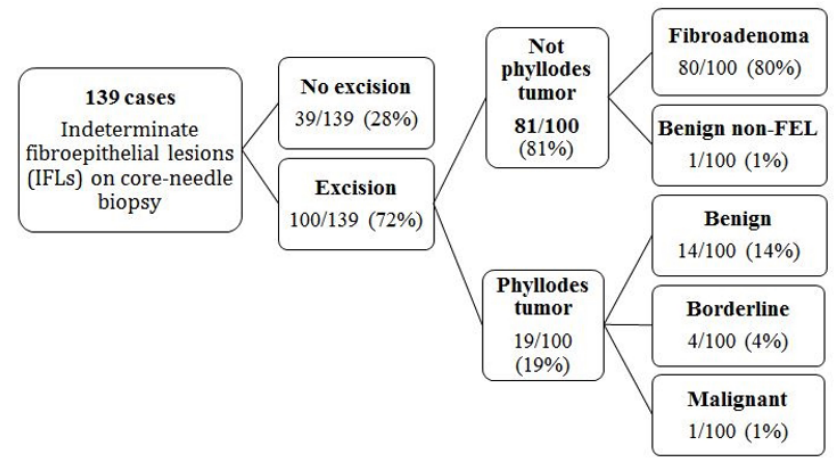

Conclusions: IFLs on breast CNB are highly unlikely to be malignant. Short-term recurrences are rare. Unnecessary surgical procedures may be avoided with a more conservative approach to these lesions (e.g., 6 month imaging follow-up), particularly in younger patients.

\section{Microinvasive Carcinoma; High Grade-HER2 Pathway Associated with High Tumor Infiltrating Lymphocytes}

Michi Morita, Rin Yamaguchi, Maki Tanaka, Susumu Eguchi, Hirohisa Yano. Kurume University Medical Center, Kurume, Japan; Kurume University School of Medicine, Kurume, Japan; JCHO Kurume General Hospital, Kurume, Japan; Nagasaki University Graduate School of Biomedical Sciences, Nagasaki, Japan.

Background: The interaction between tumors and the immune system has been attracting interest in breast cancer research. While tumor immunity must be involved in tumor progression and regression from the very early stage of tumorigenesis, no detailed study has been reported on the relationship between "early-stage" breast cancer and tumor infiltrating lymphocytes (TIL). In this study, we focused on microinvasive carcinoma, which is considered the first step of invasive carcinoma, and aimed to investigate the relationship between histological tumor factors and immunity in "early" breast cancer.

Design: Of 2593 resected breast carcinomas between 2007 and 2014, 46 (1.8\%) patients diagnosed with microinvasive carcinoma were included in this study. The relationships between the characteristics relating to tumor factors (i.e.; grade, histological type, positivity of ER and HER2, comedo) and characteristics relating to tumor immunity (TIL, healing) were examined. The histological type was divided into "cluster-like," which consisted of a small number of cancer cells and "non-cluster-like," which could be classified according to the WHO classification of tumors.

Results: As for subtype, ER ${ }^{+}$HER2-, ER ${ }^{+}$HER2 ${ }^{+}$, ER-HER2 ${ }^{+}$, and ER-HER2- accounted for $58.7 \%, 8.7 \%, 28.3 \%$, and $4.3 \%$, respectively. Compared to ER ${ }^{+} H E R 2$,

ER $^{-} \mathrm{HER}^{+}$was found to have a significantly stronger association with Van Nuys classification group $3(92.3 \%)$, Grade $3(92.3 \%)$, comedo necrosis $(100 \%)$, high TIL $(100 \%)$, and healing $(76.9 \%)(P<0.001)$. Compared to non-cluster-like invasion, the cluster-like cases showed significantly higher rates of HER2 positivity $(69.2 \%)$ and high TIL $(92.3 \%)(P<0.001)$, and tended to have more healing cases $(P<0.343)$. Conclusions: The present study revealed that HER2-positive microinvasive carcinoma has the following characteristics: 1) high-grade; 2) comedo necrosis; 3) high-TIL; 4) healing; 5) cluster-like invasion. A possible explanation for the cluster-like invasion might be that tumor immunity of the host leads to disruption of the duct and formation of microinvasive carcinoma. These results suggest that in the high-grade pathway, both tumor immunity and expression of HER2 play a role in the invasion.

228 Improving the Accuracy of ERBB2 (HER2) FISH Testing in Breast Cancer by Implementing a Robust Approach Encompassing Real-Time Histopathologic, and Immunohistochemistry Charactieristics: A Survey of 2000 Invasive Carcinomas

Diana Morlote, Robert Poppiti, Ariana Lopez, Hadi Yaziji. Vitro Molecular Laboratories, Miami, FL; Mt. Sinai Medical Center, Miami Beach, FL.

Background: $E R B B 2$ gene (HER2) testing in invasive breast cancer is the most common predictive molecular assay in surgical pathology. Despite numerous published trials, consensus conferences and external quality assurance programs, HER2 testing accuracy remains in question, necessitating further means to validate and verify HER2 status. This study aims to identify tumor characteristics that are potentially useful in correlating with (and thus validating) HER2 gene status. Such characteristics would then become part of the routine workup of HER 2 assessment, positively impacting testing accuracy. Design: In a sequential, multi-institutional series of 2000 invasive mammary carcinomas submitted for centralized HER2 FISH testing, we investigated the utility of tumor histopathologic and biologic parameters as predictors of HER2 status. These include histologic type, Nottingham grade, nuclear score, and tumor cytomorphology. Also, real-time evaluation of HER2 protein "IHC microdissection" was performed on all tumors. The IVD reagents included IHC EP3 rabbit monoclonal antibody (Cell Marque) and HER2 IQ FISH (Dako). The reviewing pathologists were blinded to the IHC and FISH results.

Results: 340 tumors were positive for gene amplification (17\%). IHC/FISH concordance was $99.5 \%$ and $99.8 \%$ among the FISH positive and negative tumors respectively. HER2 positive tumors exhibit two reproducible cytomorphologic findings: apocrinelike cytoplasm, and "CIN III"-like nuclear features. Additionally, while Nottingham grade I did not reliably predict negative HER2 status, nuclear score 1 showed a perfect negative predictive value $(100 \%)$. Among the evaluated histologic types, micropapillary carcinomas had a higher fraction of HER2 positive tumors. Finally, the use of "IHC microdissection" averted the potential misclassification of HER2 status by FISH by identifying 'hot spots' among $5 \%$ of tumors that show significant tumor heterogeneity. Conclusions: Real-time correlation with nuclear score, cytomorphology, histologic type and HER2 "IHC microdissection" offers a substantial improvement of IHC/FISH concordance as well as the accuracy of HER2 FISH testing by providing robust, gatekeeping tools that enhance the assay reliability. Such tools should be prospectively incorporated in routine HER2 FISH interpretation. We therefore strongly recommend that they also be included in future ASCO/CAP HER2 guidelines recommendations.

\section{Luminal Androgen Receptor (LAR) Subtype Breast Cancers Are} Not Histologically Defined by Apocrine Morphology

Ann M Moyer, Xuemei Wu, Daniel W Visscher, Krishna R Kalari, Kevin J Thompson, Xiaojia Tang, Vera J Suman, Travis J Dockter, Sarah A McLaughlin, Alvaro MorenoAspitia, Donald W Northfelt, Richard J Gray, Katie N Jones, Amy L Conners, Eric D Wieben, Liewei Wang, Richard M Weinshilboum, Matthew Goetz, Judy C Boughey. Mayo Clinic, Rochester, MN; Mayo Clinic, Jacksonville, FL; Mayo Clinic, Scottsdale, AZ. Background: Triple-negative breast cancer (TNBC) encompasses a wide spectrum of disease phenotypes. Based on gene expression profiling, "luminal androgen receptor" (LAR) tumors have been identified as a subset of TNBC characterized by androgen receptor (AR) signaling and expression of downstream AR targets and coactivators. Histologically, a subset of TNBC with apocrine morphologic features is known to express the AR and these are assumed to represent the LAR subtype. Because LAR tumors (characterized genomically) exhibit different behavior and treatment response as compared to other TNBC, it is important to understand the relationship between the LAR subtype and the histologic apocrine phenotype.

Design: Diagnostic needle core biopsies [pre-neoadjuvant chemotherapy (NAC)] and surgical resection specimens (post-NAC) from women enrolled in the Breast Cancer Genome-Guided Therapy (BEAUTY) study were reviewed. Our group developed a shrunken centroid model to identify LAR tumors using publically available RNASequencing datasets, which was applied to classify LAR tumors in the BEAUTY cohort. Histologic review, including immunohistochemical staining for AR, was performed on tumors identified as being of the LAR subtype.

Results: The LAR subtype comprised $16 \%(7 / 42)$ of the TNBC group and only $1 / 7$ experienced a pCR. Diagnostic biopsy specimens were available for review from 5 , while resection specimens were available for the 6 tumors with residual disease after NAC. Only 1 of 6 LAR tumors demonstrated classic apocrine morphology, while 3 additional cases showed minimal apocrine features and 2 did not show any features consistent with apocrine morphology. All tumors demonstrated positive AR staining.

\begin{tabular}{|c|c|c|c|c|c|c|c|c|}
\hline Patient & $\begin{array}{l}\text { Aege at } \\
\text { Dabrosis }\end{array}$ & Specimen & Morphologit features & ER (baserines) & 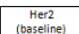 & 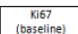 & 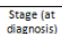 & RCB \\
\hline \multirow{2}{*}{1} & \multirow{2}{*}{52} & biopsy & Cassicl apocrine morphology & \multirow{2}{*}{$<1$} & \multirow{2}{*}{0} & \multirow{2}{*}{${ }_{11}$} & \multirow{2}{*}{ T2Nonanx } & \multirow{2}{*}{ " } \\
\hline & & resection & Classic apocrine morphology & & & & & \\
\hline \multirow{2}{*}{$2^{2}-100$} & \multirow{2}{*}{57} & biopsy & $\begin{array}{l}\text { bodrefriline apeccine w/secterory } \\
\text { festures }\end{array}$ & \multirow{2}{*}{$<1$} & \multirow{2}{*}{$1+$} & \multirow{2}{*}{30} & \multirow{2}{*}{ тзмомо } & \multirow{2}{*}{ "II } \\
\hline & & resection & $\begin{array}{l}\text { bordertine apoccine w/ marlied } \\
\text { treatnente effect }\end{array}$ & & & & & \\
\hline \multirow{2}{*}{3} & \multirow{2}{*}{46} & biopsy & $\begin{array}{l}\text { clear cell featuress, no opocrine } \\
\text { features }\end{array}$ & \multirow{2}{*}{$<1$} & \multirow{2}{*}{0} & \multirow{2}{*}{${ }_{30}$} & \multirow{2}{*}{ твмомо } & \multirow{2}{*}{ " } \\
\hline & & resection & $\begin{array}{l}\text { squamold teastures; no poocrine } \\
\text { features }\end{array}$ & & & & & \\
\hline \multirow[t]{2}{*}{4} & \multirow[t]{2}{*}{54} & biopsy & partial apocrine w/ basal features & \multirow{2}{*}{ 1:10 } & \multirow{2}{*}{$1+$} & \multirow{2}{*}{90} & \multirow{2}{*}{ T2N1MO } & \multirow{2}{*}{1} \\
\hline & & resection & partial apoccine & & & & & \\
\hline \multirow{2}{*}{5} & \multirow[b]{2}{*}{60} & biopsy & not availbble & \multirow[b]{2}{*}{ 1:10 } & \multirow[b]{2}{*}{ not vavilable } & \multirow[b]{2}{*}{ not avaliable } & \multirow[b]{2}{*}{ Tзм1м0 } & \multirow[b]{2}{*}{ "I" } \\
\hline & & resection & 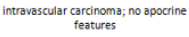 & & & & & \\
\hline \multirow{2}{*}{6} & \multirow{2}{*}{48} & biopsy & partial apocrine features & \multirow[b]{2}{*}{${ }_{1}$} & 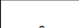 & $n$ & tovima & \\
\hline & & resection & 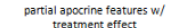 & & 0 & 29 & & " \\
\hline 7 & 47 & biopsy & not ovalibble & $<1$ & 。 & 50 & tinomo & 。 \\
\hline
\end{tabular}

Conclusions: Although breast tumors with apocrine morphology show high AR expression, conversely, LAR subtype breast tumors do not necessarily demonstrate apocrine morphology. Therefore, within the triple negative subset of breast cancer, detection of the LAR subtype requires molecular profiling, as histologic evaluation alone is not sufficient.

230 Cytologic Characteristics of Resistant Locally Advanced Breast Carcinoma after Neoadjuvant Treatment

Ann M Moyer, Daniel W Visscher, Vera J Suman, Travis J Dockter, Krishna R Kalari, Donald W Northfelt, Sarah A McLaughlin, Alvaro Moreno-Aspitia, Richard J Gray, Judy C Boughey, Matthew Goetz. Mayo Clinic, Rochester, MN; Mayo Clinic, Scottsdale, AZ; Mayo Clinic, Jacksonville, FL.

Background: Neoadjuvant chemotherapy (NAC) is often used for breast cancer with high risk tumor biology. Residual disease after NAC is associated with poor survival. Although it is known that NAC can alter tumor morphology, the type and degree of changes vary and their clinical significance remains unknown.

Design: 132 women with breast cancer were enrolled in the Breast Cancer GenomeGuided Therapy (BEAUTY) study, a NAC study consisting of 12 weeks of paclitaxel 
\pm trastuzumab and 4 cycles of anthracycline-based chemotherapy followed by surgical resection. Diagnostic needle core biopsies (pre-NAC) and resection specimens (postNAC) were reviewed to detect chemotherapy-associated changes, including cytomegaly, cytoplasmic vacuolization, histiocytoid appearance, and apocrine/eosinophilic appearance. The relationship between cytologic changes and residual tumor cellularity and Ki67 was assessed.

Results: Of the 132 patients, 45 had a pathologic complete response, 4 did not have surgery, and 83 had residual disease ( 77 available for review). Cytologic features observed included: cytomegaly $(36 ; 46.8 \%)$, cytoplasmic vacuolization $(58 ; 75.3 \%)$, histiocytoid appearance $(11 ; 14.3 \%)$, apocrine/eosinophilic appearance $(7 ; 9.1 \%)$; or no features of treatment effect $(10 ; 13.0 \%)$. A subset of tumors $(24 ; 31.2 \%)$ had a previously undescribed appearance characterized by cells arranged in clusters with stromal retraction (CSR). Tumors with CSR had higher cellularity at resection than tumors without CSR ( $37.5 \%$ vs. $20 \%$; Wilcoxon $\mathrm{p}=0.030)$, but there was no difference in proportion of cases with low Ki67 $(<15 \%)$ between those with or without CSR (Fisher $\mathrm{p}>0.07)$. None of the other cytologic features were associated with cellularity (all Wilcoxon $\mathrm{p}>0.55$ ) nor Ki67 (all Fisher $\mathrm{p}>0.07$ ).

\begin{tabular}{|c|c|c|c|c|c|c|}
\hline $\mathbf{n}(\%)$ & $\begin{array}{c}\text { Luminal A } \\
(\mathbf{n}=10)\end{array}$ & $\begin{array}{c}\text { Luminal B } \\
(\mathbf{n = 3 1})\end{array}$ & $\begin{array}{c}\text { Luminal } \\
\text { Unknown } \\
(\mathbf{n = 2})\end{array}$ & $\begin{array}{c}\text { Triple } \\
\text { negative } \\
(\mathbf{n}=16)\end{array}$ & $\begin{array}{c}\text { ER+/Her2+ } \\
(\mathbf{n}=11)\end{array}$ & $\begin{array}{c}\text { ER-/HER2+ } \\
(\mathbf{n}=7)\end{array}$ \\
\hline Cytomegaly & $2(20 \%)$ & $\begin{array}{c}17 \\
(54.84 \%)\end{array}$ & $0(0 \%)$ & $\begin{array}{c}7 \\
(43.75 \%)\end{array}$ & $5(45.45 \%)$ & $5(71.43 \%)$ \\
\hline $\begin{array}{c}\text { Cytoplasmic } \\
\text { vacuolization }\end{array}$ & $4(40 \%)$ & $\begin{array}{c}26 \\
(80.65 \%)\end{array}$ & $2(100 \%)$ & $12(75 \%)$ & $\begin{array}{c}10 \\
(90.91 \%)\end{array}$ & $5(71.43 \%)$ \\
\hline $\begin{array}{c}\text { Histiocytoid } \\
\text { appearance }\end{array}$ & $4(40 \%)$ & $2(6.45 \%)$ & $1(50 \%)$ & $0(0 \%)$ & $2(18.18 \%)$ & $2(28.57 \%)$ \\
\hline $\begin{array}{c}\text { Apocrine/eosinophilic } \\
\text { appearance }\end{array}$ & $1(10 \%)$ & $\begin{array}{c}5 \\
(16.13 \%)\end{array}$ & $0(0 \%)$ & $0(0 \%)$ & $0(0 \%)$ & $1(14.29 \%)$ \\
\hline CSR & $2(20 \%)$ & $\begin{array}{c}13 \\
(41.94 \%)\end{array}$ & $1(50 \%)$ & $2(12.5 \%)$ & $4(36.36 \%)$ & $2(28.57 \%)$ \\
\hline No treatment effect & $3(30 \%)$ & $3(9.68 \%)$ & $0(0 \%)$ & $\begin{array}{c}3 \\
(18.75 \%)\end{array}$ & $1(9.09 \%)$ & $0(0 \%)$ \\
\hline
\end{tabular}

Conclusions: While most cytologic features of NAC-treated breast tumors did not correlate with treatment response as characterized by cellularity or low Ki67, CSR, a previously undescribed feature, was associated with higher residual tumor cellularity. Given the prognostic importance of residual disease post-NAC, further studies in larger cohorts are necessary to identify whether variability in these morphologic changes affects long term clinical outcomes.

231 Utilizing Mammography of Breast Resection Specimen Slices for Lesion Identification Improves Sampling Efficacy, Accurate Assessment of Tumor Pathologic Parameters, Margin Assessment and Decrease in Average Overall Turn-Around Time

Jeffrey Mueller, Raveena Reddy. University of Chicago, Chicago, IL.

Background: The number of sections submitted for histologic examination from breast excision specimens varies significantly depending on gross and imaging findings and specimen volume. When a distinct lesion is not readily identified on gross examination, oversampling of tissue often results. If the lesion is misidentified, additional sections may subsequently need to be submitted, resulting in a delay in turn-around time and frequent problems with orientation of residual tissue. Our objective was to assess the advantages of incorporating imaging of breast excision specimens at the time of grossing with respect to lesion identification, sampling efficacy, tumor evaluation and overall turn-around time.

Design: 10 cases with biopsy proven mammary carcinoma undergoing surgical excision were selected for a limited prospective study of specimen slice imaging. Cases with predominant in situ carcinoma component were selected as these are typically difficult to identify on gross examination. Specimens were received oriented by surgeon. Grossing was performed in the conventional method with recording of dimensions, inking margins and serial sectioning. The slices were sequentially laid out and oriented on a firm plastic base and carried to the breast imaging center. Mammography was then performed on all slices. Images were immediately reviewed by a breast radiologist with the pathologist on a HOLOGIC work station. Select slices were submitted based on the imaging findings. Representative sections of uninvolved breast tissue was sampled for occult lesions. Results: In comparision with conventional method of grossing, the number of tissue blocks sampled was significantly lower, and all sections taken from the target and margins contained diagnostic information. Imaging significantly improved accuracy in measuring tumor extent and assessment of surgical margins. Lastly, none of the specimens needed additional sections submitted.

Conclusions: This imaging directed approach significantly streamlines the grossing process of select breast excision specimens with reassurance that the lesional tissue and surgical margins are more adequately sampled and accurately assessed for tumor extent and margins as well as a reduction in turn-round time.

\section{P-Rex1 Expression in Invasive Breast Cancer in Relation to} Receptor Status and Metastatic Site

Kristen Muller, Todd Miller, Jonathan Marotti. Dartmouth Hitchcock Medical Center, Lebanon, $\mathrm{NH}$.

Background: Phosphatidylinositol-3,4,5-trisphosphate-dependent Rac exchange factor 1 (P-Rex1) is an essential mediator of ErbB receptor-driven Rac responses in breast cancer cells, and is highly overexpressed in ER+ and HER2+ breast cancer models. Basal-like breast tumors lack significant expression of P-Rex1, and it is essentially nonexistent in normal mammary tissue. It has been suggested that P-Rex1 is upregulated in primary breast tumors and their metastases. The aim of this study was to evaluate the expression of P-Rex 1 in primary and metastatic human breast tumors, and to correlate expression with receptor status and metastatic site.
Design: P-Rex1 immunohistochemical expression was evaluated in 138 cases: 42 nonmetastatic primary breast tumors, 42 primary breast tumors that developed metastases, and 54 breast-derived tumors from metastatic sites. Two pathologists provided P-Rex 1 IHC histoscores based on percentage and intensity of staining; histoscores were binned into H-scores as follows: H-score 0 (histoscore 0-49); 1 (50-125); 2 (126-175); and 3 $(>175)$. P-Rex1 expression was correlated with development of metastases, receptor(ER, $\mathrm{PR}$, and HER2) status, and metastatic sites. Data is presented as mean $\mathrm{H}$-score $\pm \mathrm{SD}$. Results: Two pathologists provided P-Rex1 IHC H-scores with good concordance $(\mathrm{r}=0.85)$. Tumors from metastatic sites included bone $(\mathrm{n}=24)$, brain $(\mathrm{n}=21)$, liver $(n=4)$, and lung $(n=6)$. Primary breast tumors showed significant differences in P-Rex 1 expression based on receptor status $(\mathrm{p}=0.04)$ : $\mathrm{ER}+(1.7 \pm 1.1)$ and HER2+ primary breast tumors $(1.7 \pm 1.0)$ showed the highest P-Rex 1 expression; the lowest expression was seen in primary triple-negative breast tumors $(1.0 \pm 0.6)$. HER $2+$ metastases (from all metastatic sites) showed significantly higher P-Rex1 expression $(2.60 \pm 0.5)$ compared to other receptor subtypes $(\mathrm{p}=0.002)$. Brain $(2.1 \pm 0.94)$ and lung $(2.3 \pm 0.94)$ metastatic tumors expressed higher P-Rex1 levels compared to liver $(1.5 \pm 0.87)$ and bone metastases $(1.3 \pm 0.9)$.

Conclusions: This study corroborates the P-Rex 1 expression patterns reported in breast cancer models. P-Rex1 is overexpressed in ER+ and HER2+ breast cancers while triple negative tumors do not show significant expression. Our data also suggests that P-Rex 1 is differentially expressed in metastatic tumors based on site and receptor status. The role of P-Rex 1 in the development of breast cancer metastases warrants further investigation.

\section{Pathologic Characteristics and Outcome of Low Positive Hormone} Receptor Invasive Breast Carcinoma

Jodi S Music, Theera Rojanapremsuk, Sunati Sahoo. University of Texas Southwestern Medical Center, Dallas, TX.

Background: Testing of biomarkers in biopsies is a critical driver of determining therapy in patients diagnosed with invasive breast cancer. In 2007, ASCO/CAP determined the threshold for hormone receptor (HR) positive status to be $1 \%$ and greater in order to ensure that all patients who might benefit from hormonal treatment would be eligible. It has been shown that HR status follows a bimodal distribution, typically either very strongly positive or negative, and it is unclear how cases with immunostaining between $1 \%$ and $10 \%$, low level positive (LLP) tumors, behave, if they respond robustly to hormonal therapy, and if patient outcomes are improved with such therapy.

Design: In this retrospective study, 749 patients diagnosed with invasive breast cancer between 2006 and 2014 were reviewed. Results for HR status were analyzed on both core biopsy and excisional specimens. For 33 tumors $(4.4 \%)$ that were considered LLP for HR expression, the patient history was reviewed to determine whether hormonal treatment was given and the clinical outcome.

Results: Data from 33 patients totaling 66 samples were analyzed. Of the 33 pairs of data, 30 had one sample negative and one sample LLP, and three had both samples LLP. Twenty-seven tumors were grade III and six were grade II. None of the samples were grade I. Twenty-nine of the tumors had a Ki-67 ranging from $34 \%$ to $100 \%$, three were between $16 \%$ and $22 \%$, and one was $6 \%$.

Six patients $(19 \%)$ were treated with hormonal therapy, of which five did not have recurrence or progression, and one progressed to metastatic disease 38 months after diagnosis. Twenty-seven patients $(81 \%)$ did not receive hormonal therapy, nineteen $(70 \%)$ of which had no recurrence. Of the remaining eight patients who experienced progression, three declined any additional therapy, one was not eligible for adjuvant therapy, two progressed while receiving chemotherapy, and the remaining two patients had inconsistent follow-up and were diagnosed with metastases at eight and twelve months after initial diagnosis.

Conclusions: It is difficult to ascertain from this small cohort whether those who were treated with hormonal therapy truly benefited compared to those patients who were not treated since they had similar outcomes. Furthermore, the pathologic features of these tumors are more similar to HR-negative tumors. Additional studies, including clinical trials, need to be done in order to revise the $\mathrm{ASCO} / \mathrm{CAP}$ guidelines and possibly categorize the tumors as HR-positive, HR-negative and HR-borderline.

\section{Androgen Receptor Expression in Triple Negative Breast Cancers:} A Single Institution Experience

Palawinnage Vidarshi U Muthukumarana, Sheila E Segura, Theresa L Profeta, Ramapriya Vidhun. Danbury Hospital, Danbury, CT.

Background: Triple negative breast cancer (TNBC) is defined by estrogen receptor (ER), progesterone receptor (PR) and human epidermal growth factor receptor 2 (HER2) negativity. TNBC cannot benefit from endocrine therapy and HER-2-target therapy and known to be associated with poor prognosis. Evidence shows that androgen receptor (AR) signaling pathway plays a critical role in normal and malignant breast. We assessed the AR receptor expression in TNBC, it's clinicopathological significance and $\mathrm{AR}$ expression in non-TNBC in relation to ER/PR and HER2 positivity.

Design: The study included immunohistochemical analysis of AR expression in 36 cases of TNBC, 38 cases of ER, PR positive, Her 2 negative and 13 cases of ER/PR negative, Her2 positive breast cancers, diagnosed between January 2011 and October 2014. AR were considered positive if there were $1 \%$ or more positive invasive tumor cell nuclei staining with any degree of intensity. We evaluated the intensity of immunostaining as no staining, weak, moderate and strong and the percentage of nuclei staining as $<1 \%$, $1-10 \%, 11-50 \%$ and $51-100 \%$. A P value $<0.05$ was considered statistically significant. Results: AR was expressed with moderate to strong intensity only in 13 of 36 TNBC (36\%) patients compared to moderate to strong expression in all $38(100 \%)$ ER/PR positive, HER2 negative patients. $(\mathrm{p}<0.001)$. AR was expressed in 10 of $13(77 \%)$ HER2 positive, ER/PR negative cases. Analysis between AR expression and clinicopathologic parameters in TNBC revealed that positive AR expression was correlated with tumor necrosis $(\mathrm{p}<0.05)$. No correlation was seen with menopausal status, tumor size and 
histology, histologic and nuclear grade, lymph node status, lymphovascular invasion and stage. AR was diffusely and strongly positive in triple negative carcinomas with apocrine features.

Conclusions: AR is expressed in a significant number of TNBC and is more frequently expressed in ER/ PR positive tumors, indicating AR is a potential target for treatment in TNBC and also has a role in treatment resistant non-TNBC. The expression of $\mathrm{AR}$ is associated with some biological features of TNBC and TNBC with apocrine differentiation. Larger prospective studies are needed to further assess the prognostic value of AR in TNBC, and it's relationship of HER-2 signaling pathway in ER negative breast cancers.

\section{5 c-Myc Oncogene Highly Expressed, but Not Amplified in High} Grade Triple Negative Breast Ductal Cancers in African American Women Tammey Naab, Luisel J Ricks-Santi, Farhan Khan, Ashwini K Esnakula, Girmay Asgedom, Jeffrey Robinson. Howard University Hospital, Washington, DC; Hampton University, Hampton, VA; University of Florida, Gainsville, FL.

Background: c-Myc, a proto-oncogene, regulates expression of many target genes in cell proliferation, cell cycle regulation and apoptosis. Constitutive expression of c-Myc protein may be either due to gene amplification or increased transcriptional activity of normal gene. The study objective was to determine the correlation between immunohistochemicsl detection (IHC) of c-Myc protein and gene amplification by Fluorescence In Situ Hybridization (FISH). Furthermore, this study sought to determine the association between c-myc expression and the four major subtypes of breast carcinoma (16 Luminal A, 12 Luminal B, 6 HER2, and 24 Triple Negative), other clinicopathological factors including grade, stage, disease-free, and overall survival. Design: We analyzed 58 cases of archived breast ductal carcinoma specimens from African American (AA) women for c-Myc deregulation by FISH and IHC. Five $\mu \mathrm{m}$ sections of FFPE tumor blocks were stained with a polyclonal antibody against c-Myc (cell marque) and $\mathrm{H}$ score was derived from intensity of reactivity $(0-3)$ and percentage of reactive cells based on nuclear staining. Cases were categorized as negative (score $<$ 50 ) or positive (score $>50$ ). FISH probe mix consisted of orange directly-labeled MYC probe (CymoGen DX, NY USA) and green centromere 8 probe (Empire Genomics). Ratio $>2: 2$ was considered amplified. Pearson's correlation and McNemar's test analyzed correlation between c-Myc IHC and FISH.

Results: Positive expression by IHC correlated with TN ( $p<0.0001)$. FISH amplification correlated with HER2 positivity $(\mathrm{p}<0.0001)$. No correlation between methods was seen by subtype (Luminal A $p=0.774$, Luminal B $p=0.317$, HER $2 p=0.178$, Triple negative $\mathrm{p}=0.152)$ or between IHC and FISH expression as a whole $(\mathrm{p}=0.860)$.

Conclusions: Our previous study found high association of c-Myc IHC with triple negative breast cancer (TNBC) in AA women. Our current study finding of poor correlation between c-Myc IHC and gene amplification by FISH suggests that there might be increased transcription of c-Myc oncogene in TNBC in AA women. Evaluation of c-Myc RNA expression in TNBC would be useful in confirming this hypothesis. Recent studies have shown that loss of tumor suppressor genes such as BRCA 1 and Axin 1 are associated with enhanced c-Myc oncogenic activity. c-Myc regulatory pathways represent potential therapeutic targets for TNBC.

236 An Appraisal of Epithelial and Myoepithelial Markers in Adenoid Cystic Carcinoma of the Breast: A Unique Reverse Staining Pattern of Cytokeratin 5/6 Excludes Its Mimickers

Tokiko Nakai, Shu Ichihara, Tomonori Kawasaki, Suzuko Moritani, Akiko Kada, Noriko Ito, Tomoko Uchiyama, Hiroe Itami, Kouhei Morita, Masato Takano, Maiko Takeda, Kinta Hatakeyama, Chiho Ohbayashi. Nara Medical University, Kashihara, Nara, Japan; Nagoya Medical Center, Nagoya, Aichi, Japan; Iwate Medical University School of Medicine, Morioka, Iwate, Japan; Shiga Medical University, Otsu, Shiga, Japan; Clinical Research Center, Nagoya Medical Center, Nagoya, Aichi, Japan.

Background: Adenoid cystic carcinoma (AdCC) of the breast is an uncommon but distinct neoplasm composed of epithelial and myoepithelial cells polarized around true glandular spaces and pseudolumina, respectively. The aim of this study was to clarify whether various epithelial and myoepithelial markers can distinguish the dual cell population.

Design: The expressions of CK7, EMA, CD117, p63, calponin, CD10, S100, CK5/6, CK14 and vimentin were compared in AdCC $(n=14)$ and collagenous spherulosis $(n=5)$ using a curve created by plotting the true-positive rate (sensitivity) against the false-positive rate (1-specificity) at four threshold settings of $0 \%(0 / 1+), 10 \%(1+/ 2+)$, $50 \%(2+/ 3+)$, and $70 \%(3+/ 4+)$.

Results: The best markers for epithelial cells in AdCC were CK7 and EMA; those for myoepithelial cells were p63 and vimentin. CD10 and S100 did not act as myoepithelial markers of AdCC. CK5/6 was expressed more frequently in epithelial than in myoepithelial cells of AdCC, resulting in a reverse expression pattern analogous to that which we have recently documented in mammary adenomyoepithelioma (Virchows Arch 2015;466;191-198).

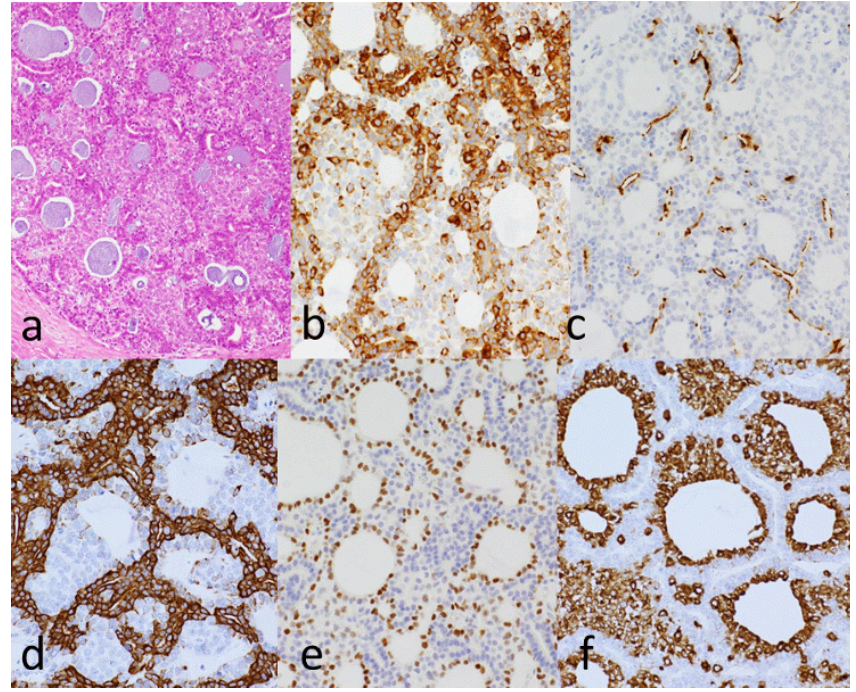

Adenoid cystic carcinoma with a cribriform pattern shows two types of structures (a). Cells around the true glandular spaces are positive for CK7 (b), EMA (c) and CK5/6 (d), while the cells around the pseudolumen are positive for p63 (e) and vimentin (f). Note that CK5/6 is a marker for epithelial rather than myoepithelial cells in adenoid cystic carcinoma.

Conclusions: Compared with myoepithelial cells in normal breast or collagenous spherulosis, neoplastic myoepithelial cells in AdCC are characterized by enhanced vimentin and attenuated CD10 and S100 expressions. Moreover, AdCC shows a unique reverse staining pattern of $\mathrm{CK} 5 / 6$, which may aid in excluding its mimickers.

237 Should Multiple Ipsilateral Invasive Breast Carcinomas Routinely Undergo Biomarker Testing?

Anupma Nayak, Ira J Bleiweiss, Bailey A Reindl, Shabnam Jaffer. Mount Sinai Hospital, New York, NY.

Background: Advances in imaging techniques have resulted in increased detection of multifocal breast carcinomas. ASCO/CAP 2013 guidelines recommend testing additional tumor foci for ER/PR/HER2 only if they show different morphologies. However, due to rapidly evolving data on tumor heterogeneity we have observed an increase in clinical requests for testing of additional tumor foci at our institution especially if the first tumor tested was negative for one of the three biomarkers. The goal of our study was to assess the clinical significance of this practice.

Design: Using our pathology database, we identified 71 patients diagnosed with ipsilateral synchronous multifocal tumors from January 2015 through September 2015. Clinical, histologic and ER/PR/HER2 profile of the tumors were recorded. ER/PR/ HER2 scores were interpreted per ASCO/CAP 2013 guidelines.

Results: Of the 71 cases, 58 were tested for additional tumor foci. Twelve $(21 \%)$ cases showed a discordant staining between the two foci for at least one of the three biomarkers. The details of the 12 discordant cases are as follows: ER discordance 7; PR discordance 6; HER2 discordance 2. Within the ER discordant cases, 3 cases showed a major variation from negative $(0 \%)$ to positive expression $(100 \%, 100 \%$ and $95 \%)$. The remaining 4 cases converted from negative $(0 \%)$ to weak positive $(2 \%, 2 \%, 5 \%$, and $5 \%$ ). Of note, $4 / 7$ cases had differences in morphology or grading. Within the PR discordant cases, 4 cases showed major variation from negative $(0 \%)$ to positive expression $(20 \%, 70 \%, 80 \%$ and $60 \%)$, whereas 2 had minor variation from negative $(0 \%)$ to weak expression $(2 \%, 2 \%) .2$ of these 7 cases had differences in morphology or grading. A difference in HER2 expression was noted in 2 cases. In 1 case only 1 of 2 invasive pleomorphic lobular carcinomas strongly expressed HER2 (75\%). In the second case, despite identical morphology of 2 poorly differentiated duct carcinomas with lymphatic invasion, 1 was ER+/ HER2+ and the other was triple negative. Interestingly, the lymphatics of the latter tumor showed HER2+ tumor emboli while being negative in the second tumor.

Conclusions: Nine $(16 \%)$ patients (7 ER+ and 2 HER2+) had changes in treatment plan due to testing of additional tumor foci. While the majority of similar appearing carcinomas may have a similar IHC profile, there may be exceptions as seen in 2 of our cases with drastic variation in HER2 expression, ultimately affecting the treatment options. Thus we propose that negative values expressed in the primary tumor be repeated routinely on additional ipsilateral tumors.

238 Validation of Modified Magee Equation to Predict the Oncotype DX Recurrence Score

Cameron Neely, Jing Yang, Shristi Bhattarai, Limin Peng, Uma Krishnamurti, Ritu Aneja, Xiaoxian Li. Emory University School of Medicine, Atlanta, GA; Rollins School of Public Health, Emory University, Atlanta, GA; Georgia State University, Atlanta, GA. Background: Estrogen receptor-positive (ER+) breast cancer has a better prognosis than HER2-positive (HER2+) or triple-negative breast cancer. Not all ER+ breast cancers need chemotherapy. Oncotype DX (ODX) is a widely used expensive molecular test to predict distant recurrence of ER+ breast cancers, and its recurrence score is used to determine if chemotherapy is necessary. Klein and Dabbs et al., from Magee-Womens 
Hospital, developed equations using routinely assessed clinicopathological parameters to predict ODX recurrence score. The aim of this study is to compare the modified Magee equation 3 (ME3) recurrence score with the ODX recurrence score.

Design: A total of $324 \mathrm{ER}+$, lymph node-negative breast cancer cases with ODX recurrence score were retrieved. Of the 324 cases, 239 cases had available information of tumor Nottingham histologic score and stage, biomarker status of ER, progesterone receptor (PR) and HER2, as well as Ki-67 index. We correlated the ME3 recurrence score with the ODX recurrence score. Other Magee equations will be evaluated once additional information, such as tumor size, is collected.

Results: The overall concordance between the ME3 recurrence score and the ODX recurrence score is $61.9 \%$ with Cohen's weighted Kappa coefficient of 0.40 ( $p<.0001)$ and Pearson's correlation coefficient of $0.56(\mathrm{p}<.0001)$. The two-step discordance is $2.5 \%$. If the intermediate groups are excluded, the concordance is $94.3 \%$ with a Kappa coefficient of $0.72(\mathrm{p}<.0001)$, suggesting a substantial agreement. Of the 22 cases with high ME3 estimated score, $4(18.2 \%)$ had low ODX score. Of the 118 cases with low ME3 estimated score, 2 (1.7\%) had high ODX score.

\begin{tabular}{|l|l|l|l|l|}
\hline $\begin{array}{l}\text { ME3 recurrence score } \\
\text { (RS) }\end{array}$ & $\begin{array}{l}\text { ODX RS } \\
\text { high }\end{array}$ & $\begin{array}{l}\text { ODX RS } \\
\text { intermediate }\end{array}$ & $\begin{array}{l}\text { ODX RS } \\
\text { low }\end{array}$ & Total \\
\hline high & 9 & 9 & 4 & 22 \\
\hline intermediate & 14 & 49 & 36 & 99 \\
\hline low & 2 & 26 & 90 & 118 \\
\hline Total & 25 & 84 & 130 & 239 \\
\hline
\end{tabular}

Conclusions: The estimated recurrence score from ME3 moderately agrees with the ODX recurrence score. When the intermediate category is eliminated, they have a substantial agreement. Cases with high or low ME3 estimated scores are not drastically different from the ODX recurrence score category. This is especially true in cases with a low ME3 estimated score.

239 Reactive Squamous Metaplasia in the Breast: A 20-Year Review Emily R Nelson, Pedram Argani, Ashley Cimino-Mathews. Johns Hopkins, Baltimore, MD.

Background: Reactive squamous metaplasia in the breast can be a diagnostic pitfall in the differential of metaplastic (sarcomatoid) carcinoma. However, diagnostic criteria to distinguish between these entities remain poorly defined. Here, we perform a retrospective review of reactive squamous metaplasia in breast core needle biopsies and excisional specimens.

Design: A search of the Johns Hopkins Department of Pathology database was performed for all cases from 1993-2013 with "breast and squamous and metaplasia" or "breast and squamous and reactive" in the diagnosis lines.

Results: There were a total of 85 diagnoses of benign squamous metaplasia. Of these cases, $34.1 \%$ were sent to our Pathology Department by an outside pathologist for expert consultation. Of the benign squamous metaplasia cases, $40.0 \%$ were associated with biopsy site changes, $32.9 \%$ were associated with proliferative lesions (such as fibroepithelial lesion), and $27.1 \%$ were discovered incidentally within the contours of a normal duct. In terms of reported histologic features, $5.9 \%$ of cases exhibited cytologic atypia, $1.1 \%$ demonstrated prominent mitotic figures, and $1.1 \%$ contained atypical mitoses. In addition, $30.6 \%$ of cases had an atypical/cancer diagnosis made in the same specimen, and $56.5 \%$ of the patients have had an atypical/cancer diagnosis made at one point in time. The majority of the diagnoses of reactive squamous metaplasia were detected on resection $(72.9 \%)$ versus on core needle biopsy $(27.1 \%)$. Of the cases detected on resection, $72.6 \%$ of patients had undergone a prior ipsilateral biopsy within the last 6 months. Of those patients with a prior biopsy, $75.5 \%$ had an atypical/cancer diagnosis on the preceding biopsy.

Conclusions: Most cases of reactive squamous metaplasia are detected in biopsy site changes or proliferation lesions on excisional specimens in patients undergoing resection for an atypical/malignant lesion diagnosed on core needle biopsy. Nearly all cases lack cytologic atypia and prominent or atypical mitotic figures. The difficulty in classifying squamous lesions in the breast is evidenced by the fact that many of these cases were interdepartmental consultations. Definitively classifying a squamous proliferation as reactive, benign metaplasia may be less diagnostically ambiguous on an excision than a core biopsy, as the entire context of the lesion is apparent on excision and only partially represented on a needle core. Future studies are underway to compare the features of reactive squamous metaplasia to atypical squamous proliferations specifically seen on core needle biopsy.

\section{Adrogen Receptor (AR) Expression in Resected Ductal Carcinoma} In Situ (DCIS) of Breast

Laila Nomani, Olaronke O Oshilaja, Benjamin C Calhoun, Christine N Booth, Charles $D$ Sturgis. Clevleland Clinic, Cleveland, $\mathrm{OH}$.

Background: Mammary DCIS is a neoplastic epithelial proliferation confined to the ductal-lobular microanatomy that is characterized by an inherent but not obligate tendency for progression to invasive carcinoma. DCIS is classically treated by combinations of excision, radiation, and adjuvant tamoxifen based upon the specific needs of individual patients. Estrogen receptor (ER) status is predictive of benefit from tamoxifen. The potential impact of androgens on mammary carcinogenesis has been studied in recent years, and several authors have conceptualized therapeutic androgen receptor targeting for AR positive invasive / metastatic disease in "triple negative" patients. Comparatively little has been published on AR in DCIS.

Design: AR immunohistochemistry (IHC) testing was performed on archival tissue blocks from 116 adult female patients who had each undergone resection of DCIS (either mastectomy or lumpectomy) at Cleveland Clinic. Only patients with "pure" DCIS were included in the data set. Exclusion criteria included invasion/microinvasion,
DCIS with metastatic disease in lymph nodes, Paget's disease of the skin/nipple, neoadjuvant therapy, and intracystic papillary carcinomas or DCIS involving papillomas. Demographic data were recorded, as were grade, ER/PR status, histologic subtypes, and the presence of apocrine differentiation. AR IHC [AR441 Dako, dilution 1:100] was interpreted by a single pathologist (cds) with a threshold of $10 \%$ immunoreactivity indicating a positive result.

Results: 116 resected DCIS cases (each from a unique patient, mean age 59 yrs, range $25-90 \mathrm{yrs}$ ) were studied. The set was comprised of $6 \%$ grade $1,27 \%$ grade 2 , and $67 \%$ grade 3 disease. Of the 116 cases, 27 (23\%) were shown to express AR at or above the $10 \%$ positive threshold. The mean age for the AR positive group was 56 yrs (range $25-73 \mathrm{yrs}$ ). AR expression was seen across all grades ( $4 \%$ grade $1,44 \%$ grade 2 , and $52 \%$ grade 3 ) but was most common in high grade DCIS and in cases with apocrine features. Of the 27 positive AR cases, 10 (37\%) were ER/PR negative, corresponding to $9 \%(10 / 116)$ of all patients.

Conclusions: AR immunoreactivity is demonstrable by IHC in $23 \%$ of resected DCIS cases. In this investigation, $37 \%$ of AR positive DCIS cases were ER/PR negative. Phase II clinical trials evaluating AR antagonists in invasive / metastatic triple negative breast cancer are currently underway, and other trials comparing anastrazole to the classical treatment of tamoxifen in DCIS are also ongoing. The potential for adjuvant therapy with AR antagonists in patients with AR positive and ER negative DCIS may be worthy of consideration.

\section{Clinically Advanced and Metastatic Mucinous Carcinoma of the} Breast: A Comprehensive Genomic Profiling Study

Sahar Nozad, Kai Wang, Siraj M Ali, Ann B Boguniewicz, Depinder Khaira, Julia A Elvin, Jo-Anne Vergilio, James Suh, Vincent A Miller, Philip J Stephens, Jeffrey S Ross. Albany Medical College, Albany, NY; Foundation Medicine Inc., Cambridge, MA. Background: Mucinous breast carcinoma (mucBC) typically features a favorable prognosis, although rarely mucBC pursues an aggressive clinical course. We queried whether comprehensive genomic profiling (CGP) would uncover clinically relevant genomic alterations (CRGA) that could lead to targeted therapies for patients with metastatic mucBC.

Design: DNA was extracted from 40 microns of FFPE sections from 22 cases of stage IV mucBC. Comprehensive genomic profiling (CGP) was performed using a hybridcapture, adaptor ligation based next generation sequencing assay to a mean coverage depth of $>550 \mathrm{X}$. The results were analyzed for all classes of genomic alterations (GA) including base substitutions, insertions and deletions, select rearrangements, and copy number changes. Clinically relevant genomic alterations (CRGA) were defined as those identifying anti-cancer drugs on the market or in registered clinical trials.

Results: Sequenced samples were from breast (11), lymph nodes (3), chest wall (2), liver (2), soft tissue (2), bone (1) and pleura (1). The median age of the 22 mucBC patients was 57 years (range 32 to 79 years). 3 mucBC were grade 1,17 were grade 2 and 2 were grade $3.21(95 \%)$ mucBC were ER+, $19(86 \%)$ were PR+ and $4(18 \%)$ were HER $2+$ by IHC and/or FISH. There were $129 \mathrm{GA}$ identified on the 22 mucBC (5.9 per tumor) including $51 \mathrm{CRGA}$ with a mean of 2.3 per tumor. Amplifications of FGFR1 and ZNF703 were found in 8 of 22 cases $(36 \%)$ on the same amplicon. This enrichment of FGFR1 amplification in $36 \%$ of mucBC $36 \%$ vs $11 \%$ in 601 cases of nonmucinous $\mathrm{ER}+\mathrm{BC}$ was significant (Fisher's exact test $\mathrm{p}<0.005$ ). In comparison with non-mucBC, mucBC is predominantly $\mathrm{ER}+(\mathrm{p}<0.001)$. Other most frequently altered genes were TP53 (32\%), CCND1 and FGF3/4/19 often co-amplified together (27\%). ERBB2/HER2 alterations were found in 5 cases (23\%) including amplifications on all 4 HER 2 + cases by IHC/FISH, and ERBB2 substitution D769Y on one additional mucBC. Additional CRGA included PIK3CA (5), BRCA1 (1), TSC2 (1), STK11 (1), AKT3 (1), and $E S R 1$ (1). Metastatic mucBC had a higher frequency of ERBB2 alterations (23\%) than non-recurrent mucBC $(\mathrm{p}<0.001)$.

Conclusions: Metastatic mucBC had a relatively high frequency of CRGA including enhancement of $F G F R 1$ and $E R B B 2 \mathrm{GA}$ compared with non-metastatic mucBC. These findings suggests that CGP can identify a variety of known and emerging therapy targets which have the potential to improve the outcomes for patients with clinically advanced and metastatic forms of this disease.

242 Retinoic Acid Receptor Alpha Is a Positive Prognostic Biomarker in Triple-Negative Breast Cancer

Angela Ogden, Andrew Green, Mohammed A Aleskandarany, Emad Rakha, Ian O Ellis, Simak Ali, Xiaoxian Li, Uma Krishnamurti, Michelle D Reid, Padmashree CG Rida, Ritu Aneja. Georgia State University, Atlanta, GA; The University of Nottingham, Nottingham, United Kingdom; Emory University Hospital, Atlanta, GA; Imperial College London, London, United Kingdom.

Background: Retinoic acid receptor alpha (RAR $\alpha$ ) has recently emerged as an attractive therapeutic target in breast cancer because agonism of this receptor can suppress tumorigenesis and metastasis. Stimulation of RAR $\alpha$ with a retinoic acid analog suppresses mammary tumor growth and lung metastasis in an MMTV-Myc mouse model. Recently, it was shown that basal-like breast cancer cells express lower RAR $\alpha$ mRNA levels than breast cancer cells of other subtypes, although levels in triple-negative breast cancer (TNBC) are unknown. Thus, RAR $\alpha$ could serve as a positive prognostic biomarker in TNBC, a role that needs to be explored.

Design: We compared RAR $\alpha$ mRNA levels in breast carcinomas of differing molecular subtypes using the METABRIC dataset $(\mathrm{n}=1975)$. We also tested the effects of nuclear RAR $\alpha$ scores on clinical outcomes in 167 immunolabeled primary TNBC specimens. The staining intensity $(0-3+)$ of nuclei was determined, and RAR $\alpha$ nuclear scores were calculated as: $1 \times(\%$ cells $1+)+2 \times(\%$ cells $2+)+3 \times(\%$ cells $3+)$. The optimum cutpoint to stratify patients on the basis of breast cancer-specific survival (BCSS) was 
determined using X-tile. The effects of RAR $\alpha$ nuclear scores on BCSS and distant metastasis-free survival (DMFS) were determined using Kaplan-Meier analysis and Cox proportional hazards models.

Results: We discovered that RAR $\alpha$ mRA levels are about one third lower in TNBCs than non-TNBCs $(\mathrm{P}=7.6 \mathrm{E}-92)$. The optimum cutpoint in RAR $\alpha$ nuclear score was $110(\leq 110=$ low, $>110=$ high $)$. High RAR $\alpha$ nuclear score was associated with better BCSS and DMFS in Kaplan-Meier analysis ( $\mathrm{P}<0.001$ for both) and in univariate Cox regression analysis $(\mathrm{P}<0.001, \mathrm{HR}=0.32$ and $\mathrm{P}=0.001, \mathrm{HR}=0.35$, respectively). These findings were upheld in multivariate analysis controlling for age at diagnosis, tumor size, Nottingham grade, lymph node stage, basal phenotype, Ki67-labeling index, and adjuvant chemotherapy ( $\mathrm{P}<0.001$ for both; $\mathrm{HR}=0.26$ for $\mathrm{BCSS}$ and $\mathrm{HR}=0.27$ for $\mathrm{DMFS})$. Conclusions: TNBCs express lower RAR $\alpha$ mRNA levels than non-TNBCs. Furthermore, RAR $\alpha$ nuclear scores can stratify TNBCs into subgroups significantly different in outcome, which could better enable clinicians to choose which TNBC patients require more aggressive treatment.

243 High HER3-EGFR Score Predicts Aggressive Disease Course in Triple-Negative Breast Cancer

Angela Ogden, Andrew Green, Mohammed A Aleskandarany, Emad Rakha, Ian O Ellis, Xiaoxian Li, Uma Krishnamurti, Michelle D Reid, Padmashree CG Rida, Ritu Aneja. Georgia State University, Atlanta, GA; The University of Nottingham, Nottingham, United Kingdom; Emory University Hospital, Atlanta, GA.

Background: Intense interest has recently been kindled in human epidermal growth factor receptor 3 (HER3) in breast cancer. HER3 biology is relevant to triple-negative breast cancer (TNBC) because HER3 limits sensitivity to EGFR and PI3K-AKT inhibitors. Although HER3 is kinase dead, it can dimerize with other ErbB family members to activate downstream signaling. In univariate analysis, HER3 staining intensity predicts risk in TNBC. However, other scoring measures have not been assessed, such as combining HER3 with its dimerization partners EGFR and HER4 in a score, considering that HER3 cannot signal without a partner.

Design: $\mathrm{N}=131 \mathrm{TNBC}$ specimens from patients treated with combined cyclophosphamide, methotrexate, and fluorouracil chemotherapy were immunolabeled and scored. Optimum cutpoints in HER3, EGFR, and HER4 H-scores and combined HER3-EGFR and HER3-HER4 H-scores (which were calculated as the sum of the individual H-scores) based on breast cancer-specific survival (BCSS) were sought using X-tile, producing categorical variables. The effect of these scores as continuous and categorical variables on BCSS, recurrence-free survival (RFS), and distant metastasis-free survival (DMFS) were tested using Kaplan-Meier analysis and Cox models.

Results: When analyzed as continuous variables, none of the individual or combined $\mathrm{H}$-scores was a significant predictor of BCSS, RFS, or DMFS. Categorical HER3-EGFR $\mathrm{H}$-score predicted worse BCSS in univariate analysis (high vs. low: $\mathrm{p}=0.046$ ), whereas significant cutpoints were not found to categorize any other score. In multivariate analysis (adjusting for age at diagnosis, menopausal status, tumor size, Nottingham grade, lymph node stage, and basal phenotype), high HER3-EGFR H-score was associated with worse clinical outcomes (hazard ratio $[\mathrm{HR}]=3.30, \mathrm{p}=0.024$ for BCSS; $\mathrm{HR}=3.02, \mathrm{p}=0.020$ for RFS; and HR $=3.18, \mathrm{p}=0.039$ for DMFS). Intriguingly, HER3EGFR H-score was a more significant predictor of BCSS and RFS than lymph node stage ( $\mathrm{p}=0.037$ and 0.089 , respectively) or tumor size ( $\mathrm{p}=0.085$ and 0.019 , respectively). Conclusions: Assessment of HER3 and EGFR by immunohistochemistry as a combined $\mathrm{H}$-score may provide important prognostic information about the clinical course of TNBC, better enabling oncologists to identify TNBC patients who need more aggressive treatment.

\section{4}

CA20 Is a Better Predictor of Breast Cancer-Specific Survival Than CIN25

Angela Ogden, Padmashree CG Rida, Michelle D Reid, Ritu Aneja. Georgia State University, Atlanta, GA; Emory University Hospital, Atlanta, GA.

Background: Centrosome amplification (CA), the presence of overly numerous or voluminous centrosomes, underlies chromosomal instability (CIN) and aggressive tumor phenotypes such as invasiveness. Since most breast tumors exhibit amplified centrosomes, this trait may serve as a prognostic or predictive biomarker to identify patients in need of more aggressive treatment regimens or centrosome-targeted therapies. While CIN25, a surrogate of CIN, is an independent predictor of poor outcomes in multiple cancers, the prognostic or predictive contributions of CA remain unknown. Design: A CA score ("CA20") was derived as the sum of log2-transformed mediancentered expression levels of $20 \mathrm{CA}$-associated genes (specifically, AURKA, CCNA2, CCND1, CCNE2, CDK1, CEP63, CEP152, E2F1, E2F2, E2F3, LMO4, MDM2, $M Y C N, N D R G 1, N E K 2, P I M 1, P I N 1, P L K 4, U B E 2 A$, and STIL). CA20 was tested for its ability to stratify breast cancer patients from the METABRIC dataset $(\mathrm{n}=2136)$ into prognostic groups based on breast cancer-specific survival (BCSS). We compared the prognostic ability of CA2 20 with that of CIN25, the sum of the expression levels of 25 CIN-associated genes. CA20 and CIN25 were analyzed as continuous and categorical variables stratified by the mean. Kaplan-Meier plots of BCSS were compared with the log-rank test statistic, and multivariate Cox models were fitted using backwards stepwise elimination. The proportional hazards assumption was checked by plotting partial residuals against the rank of BCSS time, and in all cases these plots were approximately horizontal.

Results: High CA20 and CIN25 were both associated with worse BCSS in KaplanMeier analysis based on the log-rank test $(\mathrm{P}<1.0 \mathrm{E}-6)$. However, when CA20 and CIN25 were both entered into a multivariate model (along with covariates: age at diagnosis, Nottingham grade, stage, treatment, and triple-negative receptor status), only CA20 remained significant in the final model $(\mathrm{P}=1.0 \mathrm{E}-6$, hazard ratio $[\mathrm{HR}]=1.77$ for categorical CA20 and $\mathrm{P}<1.0 \mathrm{E}-6, \mathrm{HR}=1.051$ for continuous $\mathrm{CA} 20$ ). These results suggest that the association of CIN25 with worse BCSS can be largely explained by CA20. Indeed, we found a high correlation between these two continuous variables in linear regression $\left(\mathrm{R}^{2}=0.92, \mathrm{P}<1.0 \mathrm{E}-6\right)$

Conclusions: CA20 is a better predictor of BCSS than CIN25 and may outperform CIN25 because CA20 captures not only CIN-dependent risk but also other risk factors related to CA. CA20 may serve as a novel prognostic and/or predictive biomarker in breast cancers.

245 Intraductal Papillomas without Atypia Need Not Be Excised: A Hispanic Cohort Study

Lurmag Orta, Eugenio Marcano, Mayra Maldonado-Duran. San Pablo Pathology Group, Bayamón, Puerto Rico; University of Puerto Rico, Rio Piedras, Puerto Rico; HIMA San Pablo Hospital, Bayamón, Puerto Rico.

Background: There is controversy regarding the appropriate management of intraductal papillomas without atypia or an associated high-risk lesion that are diagnosed on core needle biopsy with concordant imaging. In areas affected by health disparity, the decision to excise or undergo observation has a significant socioeconomic impact. In order to determine if excisions are justified, we evaluated the results of excisions in our institution, which serves a Hispanic population in an underserved community. Design: A search was performed on our electronic database for all consecutive breast biopsies performed from January 2011 to August 2015 and diagnosed with a papillary lesion. Intraductal papillomas were classified as with and without atypia or an associated high-risk lesion. Cases with atypia or an associated high-risk lesion $(\mathrm{n}=25)$, as well as papillary carcinomas $(n=4)$, were further excluded from the study. An experienced board-certified pathologist and a dedicated breast radiologist reviewed all available histological slides and imaging, respectively.

Results: Intraductal papillomas without atypia were identified in 110 of 2347 biopsies (4.7\%), with an average age of 61 years (range 31-84). Sixty-eight presented as intraductal or cystic lesions and sampled with ultrasound guidance (12-gauge), and 42 presented with calcifications and underwent stereotactic biopsy (9-gauge). All cases had concordant imaging. Excisions were performed on 49 (45\%) cases, 55 returned to the referral institution or had no follow up information and 6 had no residual lesion on imaging. Adjacent focal atypical lobular hyperplasia was identified in $5(10 \%)$ of the 49 excisions and was considered an incidental finding. No other high-risk lesions or upgrade to carcinoma was identified. There was no significant difference in the age of patients with and without an associated high-risk lesion on excision (average $60 \mathrm{vs}$ $62, \mathrm{p}=0.8$ ). No residual papilloma was identified in 15 cases

Conclusions: Although there was an upgrade of $10 \%$, the only lesion observed was atypical lobular hyperplasia (ALH). As incidental ALH can be appropriately managed conservatively, there is no clinically significant impact in under-recognizing these lesions, provided there is close clinical and radiological follow-up in patients not undergoing excision. In a select group of patients in areas affected by health disparity, recommendation for excision in cases with radiologic-pathologic concordance and without atypia may not be warranted.

246 Stromal ColXa1 and Tumor-Infiltrating Lymphocytes Predict Response to Neoadjuvant Therapy in HER2-Positive Breast Cancer Depending on Estrogen Receptor Status

Madhu Ouseph, Alexander Brodsky, Jinjun Xiong, Dongfang Yang, Murray B Resnick, Yihong Wang. Rhode Island Hospital/ Brown University, Providence, RI; Women and Infants Hospital/ Brown University, Providence, RI.

Background: In human epidermal growth factor receptor 2-positive (HER2+) breast cancer (BC) patients; addition of trastuzumab to standard neoadjuvant chemotherapy $(\mathrm{NAC}+\mathrm{H})$ improves pathologic complete response $(\mathrm{pCR})$ as well as recurrence-free and overall survival. However, a significant number of these patients do not achieve $\mathrm{pCR}$, especially with positive estrogen receptor $(\mathrm{ER}+)$ status. The role of tumor microenvironment as a basis for this response heterogeneity is yet to be elucidated. This study was aimed at exploring the same and identifying novel biomarkers which predict treatment response to $\mathrm{NAC}+\mathrm{H}$ in $\mathrm{HER} 2+\mathrm{BCs}$.

Design: HER2 $+\mathrm{BC}$ treated with $\mathrm{NAC}+\mathrm{H}$ between 2007-2014 were identified. ER/PR and HER2 expression were classified according to the current CAP/ASCO guidelines. Pathological response to $\mathrm{NAC}+\mathrm{H}$ was assessed by the AJCC stage and residual cancer burden (RCB) score, and RCB classes 0 -I were considered good responders. Gene expression profiling done on $\mathrm{HER} 2+/ \mathrm{ER}+$ pre-NAC $+\mathrm{H}$ tumor samples revealed strong association between increased expression of collagens and reduced expression of immune-associated genes to poor pathologic response. Stromal tumor infiltrating lymphocytes (sTILs) and immunohistochemical staining of Collagen type X (ColX $\alpha 1)$ were quantitated from pre-treatment biopsies and were evaluated with treatment response in both univariate and multivariate analysis. P-values were calculated by Pearson chi-squared test.

Results: In ER+/HER2+ patients, sTILs in pretreatment samples correlated with high likelihood of achieving good pathologic response $(\mathrm{OR}=0.94, \mathrm{p}=0.001)$, while increased expression of the ColX $\alpha 1$ protein predicted poor response to $\mathrm{NAC}+\mathrm{H}(\mathrm{OR}$ $=18.9, \mathrm{p}=0.003)$. The combination of increased ColX $\alpha 1$ expression and low sTILs was significantly associated with poor response in multivariate analysis. Although, the overall proportion of good responders were higher in ER-/HER2+ patients, surprisingly no statistically significant association between sTILs, ColX $\alpha 1$ and response to NAC $+\mathrm{H}$ was observed.

Conclusions: Increased expression of stromal ColX $\alpha 1$ and low sTILs correlate with poor pathologic response and have potential utility as prognostic markers in ER+/HER2+; but not in ER-/Her2+ BCs. This study suggests that the differences in tumor:stroma interactions of HER2+/ER+ and HER2+/ER-BCs affect NAC outcomes. Further studies are warranted to validate these findings. 
247 Pitfall

Cytokeratin 7-Negative Mammary Paget's Disease - A Diagnostic

Ugur Ozerdem, Jennifer M McNiff, Fattaneh A Tavassoli. Yale School of Medicine, New Haven, CT.

Background: CK7-negative breast cancers are rare with few cases reported in the literature, about half of which had neuroendocrine differentiation. Awareness of CK7negative breast cancers is important when evaluating a carcinoma of unknown origin. It is almost universally believed that neoplastic cells in Paget's disease are CK7-positive. In fact, CK7 is an important confirmatory immunostain for diagnosis of Paget's disease. Therefore, it is important to be aware that CK7-negative Paget's disease exists, albeit extremely rare with only 2 cases reported in the literature. We encountered 2 such cases recently; and recommend the use of GATA-3 immunostain as a tangible diagnostic tool in the CK7-negative variant of Paget's disease.

Design: Two recent cases of CK7-negative Paget's disease in our practice -one without underlying intraepithelial or invasive carcinoma- prompted this study. The second case had underlying ductal intraepithelial neoplasia 3 (ductal carcinoma in situ grade 3 ). The immunoprofile of these two cases and 7 CK7-negative ductal intraepithelial neoplasia cases were compared with 7 classic (CK7-positive) Paget's disease using GATA-3 and synaptophysin immunostains.

Results: In all 9 cases of Paget's disease (2 CK7-negative and 7 CK7-positive), the neoplastic cells showed strong and diffuse nuclear immunoreactivity for GATA-3, while none had any synaptophysin immunoreactivity. As expected, the underlying ductal intraepithelial neoplasia 3 (ductal carcinoma in situ, grade3) associated with one of the CK7-negative Paget's disease cases was also CK7-negative and GATA-3-positive. Furthermore, we also observed GATA-3 immunoreactivity in 7 CK7-negative ductal intraepithelial neoplasia cases unassociated with Paget's disease.

Conclusions: CK7-negative Paget's disease and CK7-negative ductal intraepithelial neoplasias (ductal carcinoma in situ) present a major diagnostic challenge for pathologists since there is limited awareness of their existence. When there is classic Paget's morphology, GATA-3 positivity should resolve any doubts about the diagnosis in the setting of a CK7-negative neoplastic cell population.

248 Clinicopathological Features of 40 Mammary Carcinomas with Neuroendocrine Differentiation

Ugur Ozerdem, Malini Harigopal, Fattaneh A Tavassoli. Yale School of Medicine, New Haven, CT.

Background: Invasive mammary carcinomas with any neuroendocrine differentiation (IMC-NE) are rare lesions. In this study, the clinicopathological features of 40 consecutive IMC-NEs diagnosed at a single institution were analyzed.

Design: Forty IMC-NE cases diagnosed over a ten year period (2004-2015) were retrospectively analyzed. Age, gender, size, morphology, grade, lymph node status, hormone receptor status, and neuroendocrine marker expression were reviewed.

Results: All patients were females, with a mean age of 67.5 years (median: 71.5 years, range: $31-94$ years). The median tumor size was $1.5 \mathrm{~cm}$ (range : $0.1 \mathrm{~cm}-6.9 \mathrm{~cm}$ ). All tumors showed ductal differentiation, with mucin production evident in $10 \%$. Ductal intraepithelial neoplasia (ductal carcinoma in situ) was present in $65 \%$. Intraepithelial neoplasia with solid-papillary morphology was seen in $27.5 \%$ of the cases. A majority of the cases were moderately-differentiated $(57.5 \%)$ or well- differentiated $(25 \%)$. Only 7 cases $(17.5 \%)$ were poorly-differentiated; two of these showed focal chondroid metaplasia, while another had features of mammary small cell carcinoma. Lymph node metastasis was present in $12.5 \%$ cases. Frequency of lymph node metastasis -grade for grade- was comparable to infiltrating duct carcinoma of similar size and hormone receptor status using MSKCC breast cancer nomogram. Eighty five percent of the cases were ER-positive (100\% of well to moderately-differentiated cases in contrast to only $14.2 \%$ of poorly-differentiated cases). Only one ER-positive case (2.5\%) showed HER2positivity by IHC and FISH. Synaptophysin immunoreactivity was seen in $92.5 \% ; 50 \%$ had both synaptophysin and chromogranin immunoreactivity.

Conclusions: A majority of IMC-NE are well-to moderately differentiated luminal A type ductal cancers occurring in older women with $40 \%$ having mucinous differentiation and/or an associated solid-papillary ductal intraepithelial neoplasia. Also, synaptophysin-expression is more common than chromogranin expression in these tumors. Information regarding presence of neuroendocrine differentiation will assume greater significance as new neuroendocrine targeted therapies emerge. Elucidation of potential new molecular therapeutic targets in invasive mammary carcinomas with neuroendocrine features could be useful.

249 Epstein Barr Virus (EBV) in Triple Negative Breast Cancer: A Tertiary Cancer Centre Experience

Trupti Pai, Sangeeta Desai, Mamta Gurav, Prerna Walecha, Sudeep Gupta. Tata Memorial Centre, Parel, Mumbai, Maharashtra, India.

Background: Breast cancer is the most common malignancy affecting women worldwide, but its etiology is poorly understood and conventional risk factors are able to explain only a small proportion of the cases. Recently there has been a considerable interest in the possibility that triple negative breast cancers (TNBC) might be EBVassociated. The aim of our study was to detect EBV in TNBC using Epstein barr virus nuclear antigen-1 (EBNA-1) immunohistochemistry (IHC) and Epstein barr virus encoded RNA in-situ hybridization (EBER-ISH) technique.

Design: We studied the expression of EBNA-1 (Anti-EBNA-1BP2, Polyclonal, Sigma) by IHC and EBER-ISH (RISH EBER probe, Biocare, USA) in 51 histological proven TNBC cases \{Invasive breast carcinoma (IDC) which did not express estrogen receptor (ER), progesterone receptor (PR) and human epidermal growth factor receptor (HER2)\}. The nuclear expression in tumor cells was considered as positive. Considering, EBER-
ISH as gold standard, concordance between EBNA-1 IHC and EBER-ISH was assessed and association between EBER-ISH and clinicopathological parameters was analyzed using Fisher's exact test.

Results: Age range was 29-70 years (median: 48 years). All were women. Median tumor size was $3.8 \mathrm{~cm}$ (Range: $1-10 \mathrm{~cm}$ ). Upper outer quadrant was most commonly involved. Lymph nodal metastasis was seen in 67\% (28/42). Frequency of EBER-ISH positivity was $46.5 \%$ (20/43). Considering EBER-ISH as gold standard for EBV detection, concordance of EBNA-1 was $56 \%$ respectively. Although tumor size $<2 \mathrm{~cm}(\mathrm{p}=0.24)$, lymph node metastasis $(\mathrm{p}=0.7)$ and lymphovascular emboli $(0.05)$ were frequently seen in EBER-ISH negative cases, however this finding was statistically insignificant. Conclusions: A possible causal association of EBV in TNBC is suggested by high frequency of EBER-ISH positivity noted in our study. This might have therapeutic significance because of the possible role of EBV specific cytotoxic T cells in targeting EBV associated tumor cells which can be considered as a potential targeted therapy in TNBC.

250 The Mutational Landscape of Breast Adenomyoepitheliomas

Anastasios D Papanastasiou, Huei Chi Wen, Anne M Schultheis, Charlotte KY Ng, Marcia Edelweiss, Salvatore Piscuoglio, Luciano G Martelotto, Felipe C Geyer, Kathleen Burke, Maria Rosaria De Filippo, Gabriel S Macedo, Zsuzsanna Varga, Emad Rakha, Ian O Ellis, Brian P Rubin, Britta Weigelt, Jorge S Reis-Filho. Memorial Sloan Kettering Cancer Center, New York, NY; Nottingham University, Nottingham, United Kingdom; University Hospital Zurich, Zurich, Switzerland; Cleveland Clinic, Cleveland, $\mathrm{OH}$.

Background: Adenomyoepithelioma (AME) is a rare proliferative breast lesion composed of myoepithelial and epithelial cells. AMEs often have an indolent clinical course, but local recurrences, malignant transformation and distant metastases have been reported. Here we sought to define the mutational landscape of AMEs and investigate functionally the impact of recurrent likely pathogenic mutations identified in these tumors.

Design: Ten AMEs were subjected to whole-exome massively parallel sequencing (MPS, $n=7)$ or targeted capture MPS using the MSK-IMPACT assay $(n=3)$. Somatic single nucleotide variants, small insertions and deletions, and copy number alterations were defined using state-of-the-art bioinformatics algorithms. Selected genes (i.e. HRAS and $P I K 3 C A$ ) were subjected to Sanger sequencing analysis in a series of 7 AMEs in addition to the index cases. Non-tumorigenic mammary epithelial cells (i.e. MCF10A, MCF10A with the PIK3CA H1047R mutation and MCF12As), which display a 'basallike' phenotype, were utilized for 2D and 3D functional studies.

Results: 10/17 (59\%) and 12/17 (71\%) AMEs harbored HRAS Q61R hotspot mutation and $P I K 3 C A$ kinase or helical domain mutations, respectively. In 8 cases, concurrent HRAS and PIK3CA mutations were detected. All HRAS and all but one PIK3CA mutations were found to be truncal clonal events. The case with subclonal PIK3CA mutation had 2 subclonal deletions in PIK3RI (in-frame and frameshift). In vitro forced HRAS Q61R expression in MCF10A and MCF12A cells resulted in increased proliferation and transformation rates, as well as a highly disorganized growth pattern in 3D organotypic cell cultures. In PIK3CA H1047R mutant MCF10A cells, forced HRAS Q61R expression resulted in a partial loss of epithelial phenotype and acquisition of aberrant myoepithelial differentiation, including the expression of proteins related to the smooth muscle apparatus.

Conclusions: BreastAMEs harbor recurrent HRAS and PIK3CA hotspot mutations. Functional studies results are consistent with the notion that these are driver events in the pathogenesis of AMEs and may lead to the acquisition of a more overt myoepithelial phenotype.

251 Risk of Breast Cancer among African American Women with Sclerosing Adenosis and Radial Scar

Vishakha Pardeshi, Michele L Cote, Julie J Ruterbusch, Eman Abdulfatah, Woodlyne Roquiz, Baraa Alosh, Muhammad K Alsafadi, Daniel W Visscher, Sudeshna Bandyopadhyay, Rouba Ali-Fehmi. WSU, Detroit, MI; Mayo Clinic, Rochester, MN. Background: Two benign breast disease (BBD) features, sclerosing adenosis (SA) and radial scars (RS), have been shown to be associated with increased breast cancer (BC) risk primarily from studies of white women. Here, we investigate whether these associations are seen in an African American (AA) population, and describe other BBD lesions seen in biopsy from women with SA and/or RS.

Design: Benign breast biopsies (bx) from 2,798 AA women with BBD from 1997-2008 were examined for 14 benign features, and followed for subsequent BC in Detroit, MI. $1 \%(27 / 2798)$ of all women had both SA and RS, 524 women (19\%) had SA, and $76(3.0 \%)$ had RS. Risk of BC was estimated with odds ratios (OR) and $95 \%$ CI calculated from logistic regression analysis. Descriptive analysis included calculation of proportions and chi-square tests to examine differences between BBD characteristics. Results: SA was associated with a 2 fold increase in risk of $\mathrm{BC}$ after adjusting age and bx year $(\mathrm{OR}=2.14,95 \% \mathrm{CI}: 1.25,3.67)$ compared to women without SA. Risk appears attenuated when adjusted for these variables and atypical hyperplasia $(\mathrm{OR}=1.62,95 \% \mathrm{CI}$ : 0.91-2.87). Bx with SA were more likely to have apocrine metaplasia (AM), ductal hyperplasia (DH), Ca2+, cysts, duct ectasia (DE), fibrosis, columnar alterations (CA), and atypical hyperplasia $(\mathrm{AH})($ all $\mathrm{p}<0.001)$. Women with SA were more likely to have RS and less likely to have fibroadenomas $(\mathrm{p}<0.001)$. RS was associated with over a 3 fold increase in risk of $\mathrm{BC}$ after adjustment for age and bx year $(\mathrm{OR}=3.35,95 \% \mathrm{CI}$ : $1.52,7.37)$; this risk remained after adjusting for $\mathrm{AH}(\mathrm{OR}=2.67,95 \% \mathrm{CI}: 1.18,6.01)$. Bx with RS were more likely to have AM, DH, Ca2+, cysts, intraductal papillomas, CA, and $\mathrm{AH}($ all $\mathrm{p}<0.001)$. Women with RS were also more likely to have $\mathrm{SA}(\mathrm{p}<0.001)$ and less likely to have fibroadenomas $(\mathrm{p}=0.005)$. No associations were seen with lobular hyperplasia or mucocele-like lesions in either RS or SA. 


\begin{tabular}{|l|l|l|l|l|}
\hline & $\mathrm{OR}^{*}$ & $95 \% \mathrm{CI}$ & $\mathrm{OR}^{* *}$ & $95 \% \mathrm{CI}$ \\
\hline RS & 3.35 & $1.57-7.37$ & 2.67 & $1.18-6.01$ \\
\hline SA & 2.14 & $1.25-3.67$ & 1.62 & $0.91-2.87$ \\
\hline \multicolumn{4}{|l}{ *Adjusted for age \& bx yr ** Adjusted for age, bx yr \& AH } \\
\hline
\end{tabular}

Conclusions: SA and RS are associated with each other, and with many of the other features examined. Fibrosis is associated with SA but not RS, while intraductal papillomas are associated with RS but not with SA. Risk of BC is increased for AA women with BBD showing SA or RS. Thus, in addition to the established risk of atypical hyperplasia, SA and RS, alone or in combination, may improve risk prediction in this AA population.

\section{Papillomas without Atypia: Can Excision Be Spared?}

Fresia Pareja, Sandra Brennan, Melissa Murray, Zenica L Bowser, Elizabeth Morris, Edi Brogi, Adriana D Corben. MSKCC, NYC, NY.

Background: Surgical management following core needle biopsy (CNB) diagnosis of intraductal papillomas without atypia is controversial. We performed a comprehensive evaluation of a large cohort of CNBs with IDP to evaluate the rate of upgrade to carcinoma (DCIS or invasive) on excision (EXC), and factors predictive of upgrade. Design: We identified women who underwent CNB at our center between 2003 and 2013, and had a diagnosis of intraductal papilloma without atypia or carcinoma (IDP). Two pathologists reviewed all CNB and EXC slides, and 4 re-evaluated the cases with upgrade. A radiologist reviewed all imaging studies. We assessed radio-pathologic (R-P) concordance of all cases, and excluded R-P discordant CNBs.

Results: Our study population consists of 196 women with a CNB diagnosis of IDP. Median patient age was 51 years (25-85). Most patients (171; 87.2\%) underwent EXC. The upgrade rate was $2.3 \%(4 / 171)$. The upgrade lesions included 2 invasive lobular carcinomas (ILC), and 2 DCIS, each measuring $<=2 \mathrm{~mm}$. In one case DCIS involved the residual IDP and was regarded as a true upgrade. The other 3 carcinomas were $>=8$ $\mathrm{mm}$ from the residual IDP, and classified as incidental upgrades. Factors associated with upgrade were concurrent ipsilateral breast cancer $(\mathrm{p}=0.03)$, and IDP fragmentation on CNB $(p<0.05)$. The size of IDP by imaging or CNB histology was not associated with upgrade. Twenty-five (12.8\%) IDPs did not undergo EXC, and showed no changes by radiologic and clinical follow up with a median time of 23 months (6-132).

Conclusions: The risk of upgrade following radiopathologic concordant CNB diagnosis of IDP is low $(2.3 \%)$. In our study, all carcinomas found on EXC measured $2 \mathrm{~mm}$ or less. Our findings demonstrate that conservative management of IDP diagnosed at CNB with radiopathologic concordant findings is appropriate, regardless of IDP size.

\begin{tabular}{|l|l|l|l|l|}
\hline \multicolumn{5}{|l|}{ Table 1 } \\
\hline Upgraded Cases & $\# 1$ & $\# 2$ & $\# 3$ & $\# 4$ \\
\hline Case with Upgrade & 50 & 51 & 52 & 58 \\
\hline Age (y) & No & Prior & Concurrent & Concurrent \\
\hline Ipsilateral Breast Cancer & 13 & 10 & 13 & 3 \\
\hline Radiologic size (mm) & & & & \\
\hline IDP CNB Pathology & 4 & 2 & 3 & 4.5 \\
\hline Size (mm) & Yes & Yes & Yes & Yes \\
\hline Fragmentation & & & & \\
\hline Carcinoma EXC Pathology & DCIS & DCIS & ILC & ILC \\
\hline Type & 2 & 2 & 1 & 2 \\
\hline Size (mm) & 1 & $1-2$ & 2 & 2 \\
\hline Grade & In IDP & 11 & 8 & 15 \\
\hline $\begin{array}{l}\text { Distance from } \\
\text { IDP (mm) }\end{array}$ & True & Incidental & Incidental & Incidental \\
\hline Type of Upgrade & & &
\end{tabular}

253 Tumor Infiltrating Lymphocytes (TILs) in Triple Negative Breast Cancer (TNBC): A Three-Tier System Predicts Survival

Fresia Pareja, Edi Brogi, Li Fu, Zenica L Bowser, Muzaffar Akram, Larry Norton, Hannah Y Wen. MSKCC, NYC, NY; Tianjin Medical University, Tianjin, China. Background: Quantification of TILs has emerged as a prognostic factor for TNBC. TILs $>50-60 \%$ is the cutoff used in most studies, whereas the prognostic value of TILs $<50 \%$ remains uncertain. A scoring system for TILs has been proposed (Salgado, Ann Oncol, 2015), but lacks independent validation. We quantified TILs in a large cohort of clinically annotated TNBC and correlated the findings with outcome.

Design: We identified 168 consecutive women with TNBC treated at our center between 2002 and 2006. We excluded neoadjuvant cases. We quantified TILs as \% of tumor stromal area in whole tissue sections using Salgado's criteria. We tested Androgen Receptor (AR), EGFR and CK $5 / 6$ by immunohistochemistry (IHC) on tissue microarrays of the 168 TNBC. Correlation with overall survival (OS) and disease specific survival (DSS) was assessed by univariate analysis (UVA; log rank test) and multivariate analysis (MVA; Cox proportional hazard model) using SPSS software.

Results: Patient median age was 56 years (27-85). Median follow-up was 90 months (3-157). The TNBC included 131 (78\%) invasive ductal (IDC) NOS, 18 (10.7\%) IDC with features of special subtypes, $8(4.8 \%)$ apocrine, 5 (3\%) micropapillary, $4(2.4 \%)$ metaplastic, and $2(1.2 \%)$ pleomorphic lobular. Median tumor size was $2.1 \mathrm{~cm}(1-11.5)$. Most $(96 ; 57.5 \%)$ patients had negative lymph nodes, and $150(90.4 \%)$ received adjuvant chemotherapy. Survival analysis confirmed the prognostic value of TILs. A 3-tier TILs (3t-TILs) model (TILs $<10 \%$, TILs $10-59 \%$, TILs $>=60 \%)$ predicted OS $(\mathrm{p}=0.01)$ and DSS $(\mathrm{p}<0.01)$ on UVA. On MVA, the 3t-TILs was an independent predictor of OS
$(\mathrm{HR}=0.558, \mathrm{p}=0.01)$ and DSS $(\mathrm{HR}=0.352, \mathrm{p}<0.01)$. Histological type $(\mathrm{p}=0.03)$, tumor grade $(\mathrm{p}=0.02)$, and $\mathrm{AR}(\mathrm{p}=0.01)$ differed among the $3 \mathrm{t}$-TILs groups, but basal-like IHC phenotype (EGFR+ and/or CK5/6+) did not. TILs showed a positive correlation with high tumor grade, and correlated inversely with apocrine histology, and AR positivity. Conclusions: TNBC with TILs $>=60 \%$ have an excellent outcome. TNBC with TILs $10 \%-59 \%$ have significantly better OS and DSS than TNBC with TILs $<10 \%$. We propose that an outcome-based 3-tier system for TILs evaluation be further tested.
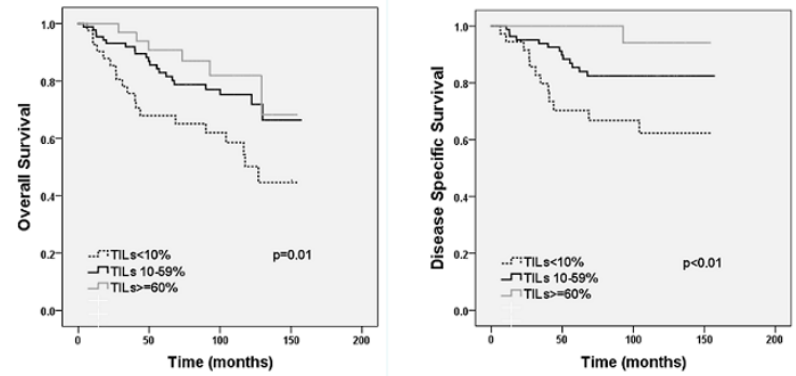

254 Evolution of Practices for HER2 Testing in Breast Cancer (BC) in France: Results of PartenHER National Survey of 109 Structures Practicing HER2 Test

Frederique Penault-Llorca, Jerome Chetritt, Bruno Poulet, Marie-Pierre Chenard, Magali Lacroix-Triki, Gaetan MacGrogan, Laurent Arnould, Jean-Pierre Bellocq. Centre Jean Perrin, Clermont-Ferrand, France; Institut d'Histo-Pathologie, Nantes, France; Institut de Pathologie de Paris, Malakoff, France; Centre Hospitalier Universitaire de Strasbourg, Strasbourg, France; Institut Universitaire du Cancer de Toulouse - Oncopole, Toulouse, France; Institut Bergonié, Bordeaux, France; 7Centre Georges François Leclerc, Dijon, France; AFAQAP, Strasbourg, France.

Background: The aim of PartenHER survey was to evaluate the evolution of French pathologists practices for HER2 test in BC throughout the past 10 years.

Design: A self-completion questionnaire of 41 items organized into 7 parts : type of structures, pre-analytical, analytical, post-analytical, difficult-to-classify cases, quality and training, was developed by a scientific committee representative of private and public sector. The survey was sent by postal mail to 450 French pathology structures (FPS) in February 2015. Some questions were covering the practices in 2015, 2010 and 2015. Results: On July $30^{\text {th }}, 118$ questionnaires have been collected. 109 FPS structures $(53 \%$ private, $47 \%$ public), regularly performing HER 2 test and representing close to 620 pathologists, completed the survey.

Up to $62 \%$ of structures declared to have a referent pathologist for HER2 in immunohistochemistry (IHC). Furthermore, in more than half of the cases, a referent pathologist was in place since more than 10 years. In 2015, the IHC technique was automated in $100 \%$ of the structures, two thirds of then being automated for 10 years. The HER2 in situ hybridization test in (ISH) was performed in $42 \%$ of the structures that responded to the survey. ISH is automated in $83 \%$ of the structures and in place for more than 10 years in $20 \%$ of the cases and for five years in $56 \%$.

Between 2005 and 2014, the median number of annual tests grew from 236 to 445 for IHC and from 97 to 215 for ISH. 31\% of FPS subcontracts HER2 IHC tests for other structures, and $52 \%$ ISH.

HER2 test was performed mainly on surgical specimens in 2005 and 2010. But the trend was reversed in 2015 (after ASCO CAP publications) with a major proportion of tests done with biopsy.

Conclusions: For numerous years, pathologists mobilized in a collective effort to optimize the quality of their practice. The PartenHER survey helps to better picture the qualitative and quantitative aspects of the French pathology practice concerning HER2 tests over 10 years. These evolutions prove to be positive in all the domains of this activity.

255 Metaplastic Breast Carcinoma with an Admixed Invasive Ductal Carcinoma Component: Does It Matter?

John M Pike, Katie Dennis, Ossama Tawfik, Fang Fan. University of Kansas Medical Center, Kansas City, KS.

Background: Metaplastic breast carcinoma (MBC) is a morphologically heterogeneous group with either non-glandular epithelial (squamous) or mesenchymal (spindle cell, chondroid, osseous, or myoid) differentiation. MBC may occur in a pure form or as mixed with an invasive ductal carcinoma component. It is not clear if pure and mixed MBCs have different clinical outcomes. In this study, we reviewed and compared the clinicopathologic features of pure and mixed metaplastic carcinomas.

Design: All cases of MBC (lumpectomy or mastectomy) in our institution between 2002 and 2015 were identified from the pathology electronic record system. A MBC is categorized as pure if more than $90 \%$ of the tumor is metaplastic and categorized as mixed if an invasive ductal carcinoma component is present and accounts for $\geq 10 \%$ of the tumor. Histologic parameters including tumor size, nuclear grade, histologic grade, and estrogen-receptor (ER), progesterone receptor (PR), and HER2 status were collected. Clinical features including lymph node status and presence or absence of distant metastasis were also collected, as well as survival.

Results: Total of $24 \mathrm{MBCs}$ were identified and slides reviewed. 6 cases were classified as pure $\mathrm{MBC}$ and 18 as mixed. Clinicopathological data and biomarker profile of these cases are shown in the following table. 


\begin{tabular}{|l|l|l|}
\hline & $\begin{array}{l}\text { Pure Metaplastic Carcinoma } \\
(\mathrm{n}=6)\end{array}$ & $\begin{array}{l}\text { Mixed Metaplastic Carcinoma } \\
(\mathrm{n}=18)\end{array}$ \\
\hline Mean Age (years) & 59 (range 45-71) & 55 (range 46-75) \\
\hline Mean Tumor Size (cm) & 6.2 (range 2.3 - 16.8) & 4.2 (range 0.0 - 24.0) \\
\hline Nuclear Grade & $1: 0$ & $1: 0$ \\
\hline & $2: 0$ & $2: 1$ \\
\hline & $3: 6$ & $3: 17$ \\
\hline Histologic Grade & All grade III & All grade III \\
\hline Lymphovascular Invasion & Present: 0 & Present: 6 \\
\hline & Absent: 6 & Absent: 12 \\
\hline Axillary Lymph Node* & $\mathrm{N}_{0}-6$ & $\mathrm{~N}_{0}-11$ \\
\hline & $\mathrm{N}_{\mathrm{la}}-0$ & $\mathrm{~N}_{\mathrm{la}}-3$ \\
\hline & $\mathrm{N}_{\mathrm{lb}}-0$ & $\mathrm{~N}_{\mathrm{lb}}-2$ \\
\hline ER/PR/HER2 Status & All ER-/PR-/Her2- & All ER-/PR-/Her2- \\
\hline Follow-up (months) & 24 (range 1 - 115) & 27 (range 13-67) \\
\hline Distant Metastases* & Yes: 1 & Yes: 5 \\
\hline & No: 5 & No: 12 \\
\hline Death* & Yes: 0 & Yes: 2 \\
\hline & No: 5 & No: 12 \\
\hline
\end{tabular}

*Complete clinical and/or pathologic data was not available for all cases

Conclusions: It is important to recognize and report the percentage of the invasive ductal carcinoma component in MBCs if present. Mixed MBCs have a higher rate of lymphovascular invasion (33\% vs. $0 \%)$ and lymph node metastases $(26 \%$ vs. $0 \%)$ as compared to pure MBCs. All MBCs (pure and mixed) are negative for ER, PR, and HER-2, with high rates of distant metastases ( $29 \%$ vs $16 \%)$.

\section{Megakaryocytes in Lymph Nodes of Patients with Breast Cancer:} A Clinicopathologic Correlation

Jennifer Pincus, Juehua Gao, Megan Sullivan. Northwestern University Feinberg School of Medicine, Chicago, IL.

Background: Megakaryocytes are rarely identified in axillary lymph nodes of women with breast cancer (BC), but when present, create a potential diagnostic pitfall. Mimicking isolated high-grade tumor cells, they can lead to an inaccurate diagnosis of metastatic carcinoma, especially in the setting of neoadjuvant chemotherapy (NAT) and most of the previous case reports detailing this finding have been this clinical setting. The etiology of the megakaryocytes in lymph nodes is unknown, but bone marrow suppression secondary to NAT, granulocyte colony stimulating factor (G-CSF) administration, or concurrent bone marrow disease have been proposed as possible causes. In this study, we sought to identify BC patients with megakaryocytes in their lymph nodes to determine if there were any common clinicopathologic characteristics. Design: Ten women undergoing surgical excision for $\mathrm{BC}$ with megakaryocytes in at least 1 lymph node were identified between 2010 to 2015 (mean age: 46.4, range 32-64 years). When possible, megakaryocytes were confirmed with cytokeratin and/ or CD61 immunohistochemistry (IHC). Electronic medical records were reviewed for patient demographics, tumor characteristics, NAT, history of hematologic disorders, medications, past history of malignancy and clinical follow-up.

Results: Megakaryocytes were confirmed by IHC in $9 / 10$ cases. In all cases, they were an isolated finding, not associated with extramedullary hematopoiesis. Nine patients had invasive ductal carcinoma (2-grade 1, 1-grade 2, and 6-grade 3) and 1 had invasive lobular carcinoma. Six patients were ER/PR positive/HER2 negative, 1 patient was ER/ PR negative/HER2 positive, and 3 patients were ER/PR/HER2 negative. Metastatic carcinoma was also present in the lymph nodes in 5 women. Two women had imaging to rule out metastatic disease with negative results. Four women $(40 \%)$ received NAT (doxorubicin, cyclophosphamide, and paclitaxel) and 2 of these patients also received G-CSF. None had a history of hematologic disease or prior malignancy and no common medication usage was identified in the interval before surgery.

Conclusions: To our knowledge, this is the largest sampled population of $\mathrm{BC}$ patients with megakaryocytes in their lymph nodes. Prior single case reports have highlighted an association with NAT, however, $60 \%$ of our study population had no prior therapy. The patients did not share similar medication histories and had no known hematologic disease. Megakaryocytes in lymph nodes are a potential diagnostic pitfall of which pathologists should be aware, although the etiology remains unclear.

257 Genomic Characterization of "CDH1- Unaltered” Invasive Lobular Carcinoma of the Breast

Zheng Ping, Gene P Siegal, Shuko Harada, Dejun Shen. University of Alabama at Birmingham, Birmingham, AL.

Background: E-cadherin (CDH1) is a glycoprotein that mediates adhesion between the epithelial cells and can also suppress cancer invasion. Mutation or deletion of the CDH1 gene has been reported in $30-60 \%$ cases of invasive lobular carcinoma (ILC). However, little is known about the genomic features of ILC without a CDH1 mutation (CDH1-Unaltered). The aim of this study is to characterize molecular changes in ILC without a CDH1 mutation.

Design: Whole genome sequencing data of 169 ILC cases were analyzed. Specific genetic and epigenetic abnormalities were compared between the "CDH1-Altered" and "CDH1-Unaltered" groups. All sequencing data and corresponding pathology information were obtained from The Cancer Genome Atlas (Invasive Breast Cancer, TCGA provisional) and were analyzed via the cBioPortal bioinformatics tools.

Results: CDH1 gene was altered in 98 cases of ILC (58\%). There was no significant difference for any of the demographics (patient age, menopause status) or clinical and pathologic characteristics, including histologic grade, tumor stage, lymph node metastases, and ER/RR/Her2 status between "CDH1-Altered" and "CDH1-Unlatered" groups, suggesting a minimal confounding bias between them. No prevalent gene mutation was found in the "CDH1-Unlatered" ILC. However, amplification of three genes (HOOK3, IKBKB, and DKK4) on 8p11 and CDK12 gene on 17q12 were found more frequently in the "CDH1-Unlatered" ILC compared with their "CDH1-Altered" counterparts $(11.3 \%$ vs $2.1 \%$ for 8 p 11 and $9.9 \%$ vs 2.1 for $17 \mathrm{q} 12$, p $<0.05)$. In addition, ERBB3 and CTNNB1 proteins were found to have higher expressions by Reverse Phase Protein Array (RPPA) in the "CDH1-Unaltered" group.

Conclusions: Our study suggests that gene amplification at chromosome 8p11 or 17q12, and/or protein upregulation of ERBB3 or CTNNB1 appear to play important roles in the pathogenies of "CDH1- Unaltered" ILC.

258 Extracapsular Extension and Lymphovascular Invasion in Breast Cancer Sentinel Lymph Nodes Is Associated with Non Sentinel Lymph Node Metastases

Ana S Pires-Luis, Joao Lobo, Liliana Santos, Margarida Caldas, Carla Bartosch, Conceicao Leal. IPO-Porto, Porto, Portugal; ICBAS-UP, Porto, Portugal.

Background: Extracapsular extension (EE) in breast cancer axillary lymph nodes has been associated with a worse prognosis. Additionally, primary tumor lymphovascular invasion (LI) has been related to positive axillary lymph nodes and poor outcome. Even though EE in sentinel node (SN) has been associated with a higher number and percent of metastases in non sentinel lymph nodes (NSLN), the role of LI in SN is not yet explored. Thus, we aim to assess if EE and LI in SN is associated with a higher risk of metastases in NSLN.

Design: We reviewed primary tumor features, SN and LN status of 188 breast cancer patients with SN metastases that underwent axillary lymph node dissection between 2008 and 2010 at our institution. We evaluated EE, LI (capsular and in perinodal adipose tissue) and size of SN metastases, and studied its association with metastases in NSLN, using Chi-square and multivariate logistic regression [OR $(95 \% \mathrm{CI})$ ] as appropriate. Results: Nearly half of patients had ductal (NST) carcinoma ( $\mathrm{n}=93,49.5 \%), 14(8 \%)$ lobular carcinoma and 27 (14\%) micropapillary carcinoma (pure or mixed); most tumors were grade $2(n=109,58 \%)$, followed by grade $3(n=61,33 \%)$ and grade $1(n=17,9 \%)$; LI was observed in $112(60 \%)$ primary tumors.

A total of $50(27 \%)$ patients with SN metastases had EE; LI was present in $25(13.5 \%)$ and SN metastases were $>1 \mathrm{~cm}$ in $79(42 \%)$. NSLN metastases were present in 103 $(54.8 \%)$ patients, having $>3$ lymph nodes involved in $13(18.6 \%)$.

Sentinel lymph node EE, LI and combined EE/LI were associated with metastases in NSLN ( $p=0.013, p=0.03$ and $\mathrm{p}=0.007$ respectively).

In multivariable logistic regression analysis, combined EE/LI in SN [OR: 2.14 (1-4.57)] and size of SN metastases [OR: $5.03(2.5-10.14)$ ], controlling for subtype, grade and LI in primary tumor, were significantly associated with metastases in NSLN.

Conclusions: Lymphovascular invasion in SN, in addition to extracapsular extension and size of metastases should be considered in breast cancer pathology reports. Further studies are warranted to assess the prognostic relevance of EE and LI in SN. This might be important for tailoring therapeutic strategies, namely axillary lymph node dissection and adjuvant treatment in selected patients.

259 Clonal Relationship among Synchronous Ipsilateral Fibroepithelial Lesions: Progression from Fibroadenomas to Phyllodes Tumors

Salvatore Piscuoglio, Charlotte KY Ng, Melissa Murray, Felipe C Geyer, Marcia Edelweiss, Britta Weigelt, Edi Brogi, Jorge S Reis-Filho. Memorial Sloan Kettering Cancer Center, New York, NY.

Background: Phyllodes tumors (PTs) and fibroadenomas (FAs) are breast fibroepithelial lesions. FAs are benign, whereas the clinical behavior of PTs varies according to histologic grade (benign, borderline and malignant PTs). Malignant transformation from benign PTs to borderline and malignant PTs is a well-documented phenomenon, whereas progression from FAs to PTs, albeit possible, has not been as extensively documented. Massively parallel sequencing (MPS) allows for the detection of the clonal-relatedness. Here we employed MPS to characterize the repertoire of somatic genetic alterations of five ipsilateral fibroepithelial lesions synchronously diagnosed, and to determine their clonal relatedness.

Design: A 37 year-old female patient with 5 FAs, 1 benign PT and 1 malignant PT synchronously diagnosed in the right breast was surgically treated at our institution. DNA samples extracted from all tumors, except for $2 \mathrm{FAs}$, and matched normal tissues after microdissection were subjected to targeted capture MPS using the MSK-IMPACT platform, which targets all coding regions and selected intronic and promoter regions of 410 key cancer genes. Somatic mutations, insertions/ deletions, cancer cell fraction and clonal-relatedness were detected using state-of-the-art bioinformatics algorithms. Results: MSK-IMPACT sequencing revealed MED12 exon 2 mutations, which have been reported in the majority of FAs and PTs, in four lesions: a clonal MED12 Gly44Val mutation in one FA and in the benign PT, and a clonal MED12 Gly44Asp mutation in another FA and in the malignant PT. One of the FAs did not harbor any MED12 mutations. A clonal-relatedness analysis based on the somatic mutations detected supported the notion that the benign PT was possibly clonally related to one of the FAs, whereas the malignant PT was likely clonally related to the other FA (both $P<0.05$, binomial tests). A clonal TERT promoter $\mathrm{C} 228 \mathrm{~T}$ hotspot mutation and mutations in other bona fide cancer genes (SETD2 and $S F 3 B 1$ ) were exclusively detected in the malignant $\mathrm{PT}$, suggesting that these alterations may have played a role in the progression to a malignant phenotype.

Conclusions: The identification of multifocal ipsilateral fibroepithelial lesions harboring distinct MED12 mutations is consistent with the notion that co-existing fibroepithelial 
tumors can arise independently. Furthermore a synchronous FA and malignant PT shared an identical MED12 mutation, suggesting that the FA may have constituted the substrate for the development of the malignant PT.

260 p16 Is Highly Expressed in TNT and Is Associated with Worse Overall Survival (OS) in All Breast Cancers (BC)

Kimberly S Point du Jour, Rachel Geller, Zachary J Dureau, Yuan Liu, Cynthia Cohen, Gabriela M Oprea-Ilies. Emory University, Atlanta, GA; Winship Cancer Institute.

Background: Genetic alterations affecting p16 protein are well described in human cancers suggesting that inactivation of this pathway may be necessary for carcinogenesis in some tumor types.

We aimed to study p16 in BC by immunohistochemical (IHC) methods in a large series of hormone receptor (HR) positive and triple negative tumors (TNT) in relation with demographic, pathologic features, biomarkers and clinical outcome.

Design: Invasive mammary carcinomas (IMC) diagnosed during a 7-year period were reviewed. The IMC markers ER, PR, and Her-2 scored by the new CAP standards were included. The tumors were studied as Her-2 positive, TNT, and hormone receptor (HR) positive. Tissue microarrays (TMAs) were stained with p16. P16 positivity was correlated with, demographic, pathologic data and clinical outcome.

Results: Of the 157 IMC studied $37.6 \%$ where HR+ and $57.3 \%$ TNT. The age at diagnosis varied from $24-90$ years. P16 was overall positive in $52.2 \%$.

Of the 75 p 16 positive in AA, 12 were HR+ and $87 \%$ TNT vs. Caucasians who were $20 \% \mathrm{HR}+$ and $80 \% \mathrm{TNT}$.

$85 \%$ of TNT showed statically significant $\mathrm{p} 16$ expression $(\mathrm{p}<.001)$ and they were correlated with basal-like BC, large tumor and high grade (all<.001)

On univariate analysis, $\mathrm{p} 16$ positivity had a statically significant positive correlation with African-American (AA) race, TNT, large tumor size, high histologic grade and CK14 and it was inversely correlated with p53 (Table 1).

\begin{tabular}{|l|l|l|}
\hline \multicolumn{2}{|l|}{ p16 Positve vs. Negative 5 (N) } & p value \\
\hline Positve correlations & $94(75)$ vs. $80(60)$ & 0.01 \\
\hline AA & $85(70)$ vs 27 (20) & $<.001$ \\
\hline TNT & $66(54)$ vs $48(36)$ & 0.024 \\
\hline Tu size $>=2 \mathrm{~cm}$ & $64(52)$ vs $18(13)$ & $<.001$ \\
\hline Grade III & $68(36)$ vs $100(13)$ & 0.015 \\
\hline CK14 & $68(36)$ vs. $31(4)$ & 0.014 \\
\hline Negative correaltions
\end{tabular}

Overall survival (OS) was statistically significant worse in $\mathrm{p} 16 \mathrm{BC}(\mathrm{p}=0.0027)$, in the non-TNT $(\mathrm{p}=0.05)$ and in AA patients $(\mathrm{p}=0.001)$.

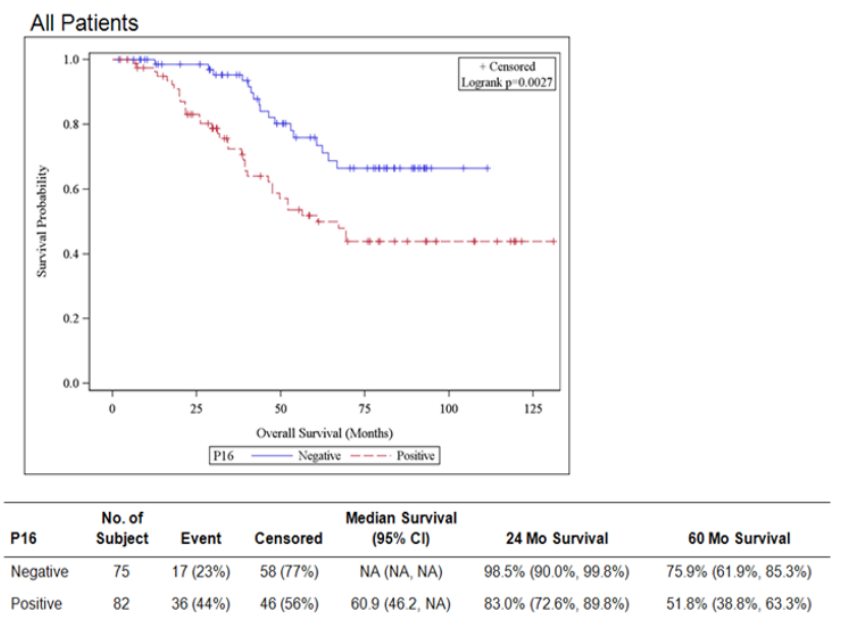

Conclusions: P16 may constitute a prognostic marker and patients with these tumors may benefit from a more aggressive therapy.

P16 expression may differentiate breast carcinoma subtypes and explain the more aggressive nature of TNT.

High frequency of p16 positivity in breast cancers of African-American women may indicate different tumor biology.

261 Analysis of MYB IHC and FISH in Breast Adenoid Cystic Carcinomas, Microglandular Adenosis and Collagenous Spherulosis

Justin Poling, Raluca Yonescu, Rajni Sharma, Yi Ning, Ashley Cimino-Mathews. Johns Hopkins Medical Institutions, Baltimore, MD.

Background: Breast adenoid cystic carcinoma (ACC) is a rare triple negative tumor that has a favorable prognosis and is generally cured with excision. Thus, histologic distinction of ACC from triple negative ductal carcinoma is essential for prognosis and treatment. The cellular origin of ACC is unclear, but early studies suggest microglandular adenosis (MGA) as a precursor to ACC. In addition, the histologic distinction of breast ACC from other benign histologic mimics, such as collagenous spherulosis (CS), can be challenging on limited sampling. ACC, MGA, and CS share overlapping immunophenotypic features; therefore, additional diagnostic tools are needed for proper diagnosis.A recurrent translocation $\mathrm{t}(6 ; 9)$ between $M Y B$ and $N F I B$ has been described in both salivary and breast ACC, and this translocation can be detected with fluorescence in situ hybridization (FISH). The presence or absence of this translocation has never been studied in MGA or CS.

Design: Pathology archives were retrospectively searched for cases of primary breast ACC, CS, and MGA. Whole sections from 12 ACC, 7 CS, and 5 MGA were labeled by IHC for MYB (Abcam ab45150, clone EP769Y at 1:100), with any nuclear labeling considered positive. Whole sections from all cases were subjected to MYB FISH using a commercially available MYB dual color break apart probe (ZTV-Z-2143-200, ZytoVision, Germany) designed to detect translocations involving the chromosomal region 6q23.3 harboring the MYB gene, and results were scored by a cytogeneticist. Clinicopathologic features including patient age, race, tumor stage, grade, size, treatment, and outcome were collected.

Results: All cases were from female patients; the median ages for patients with ACC, CS and MGA were 56 years (range, 36-69), 62 years (range, 33-75) and 64 years (range, 36-89), respectively. MYB IHC labeled $100 \%(12 / 12)$ of ACC, $57 \%(4 / 7)$ of CS, and $0 \%(0 / 5)$ of MGA cases. In cases where FISH hybridization was successful, MYB rearrangements were detected in $80 \%(8 / 10)$ of ACC, and in no cases of CS $(\mathrm{n}=6)$ or MGA $(\mathrm{n}=4)$.

Conclusions: Although all ACCs showed IHC positivity for MYB, the majority of CS cases did as well. Thus, MYB labeling alone should not be used as support for the diagnosis of ACC. FISH appears to be the preferred adjunct study, as it will detect MYB rearrangements in the majority of breast ACCs, with no false positive results seen in CS or MGA. The lack of rearrangements detected in CS and MGA cases in this limited series does not support either of these entities being precursor lesions to ACC.

262 Non-Atypical Intraductal Papillomas of the Breast Diagnosed in Core Biopsies: Are Pathologic Factors Predictive of Upgrade?

Morad Qarmali, Oluwole Fadare. UC San Diego, San Diego, CA.

Background: Surgical excision is often recommended for intraductal papillomas of the breast (IDP) diagnosed in a core biopsy based on the possibility of an associated neoplastic lesion. To provide some insights into whether excision is always necessary in this setting, we evaluated whether morphologic findings in IDP can be used to predict which cases will be upgraded in the excision and which cases will have no residual papilloma in the excision

Design: We reviewed 115 consecutive cases in which an IDP was diagnosed in a core biopsy and an excision was subsequently performed. We excluded cases of atypical IDP as well as cases with concurrent carcinoma in the same quadrant. Clinical and radiologic information were collected. Upgrade rates were determined and logistic regression analyses were used to assess which clinicopathologic features were significantly predictive of an upgrade or the absence of residual IDP in the excision. An upgrade was defined as in-situ or invasive carcinoma in the excision. Clinicopathologic characteristics that were evaluated included 1) percent of cores involved by IDP irrespective of the extent of involvement of each core 2) length of longest contiguous focus of IDP 3) necrosis 4) calcifications 5) patient age 6) symptomatology 7) biopsy-excision interval Results: $14(12.2 \%)$ of the 115 cases were upgraded in the excision. The upgrades included invasive carcinoma $(\mathrm{n}=3)$, DCIS $(\mathrm{n}=9)$, and LCIS $(\mathrm{n}=2)$. The foci of carcinoma were within $5 \mathrm{~mm}$ of the biopsy site in only $43 \%$ of cases. In $23(20 \%)$ of 115 cases, there was no residual papilloma in the excision. In our logistic regression analysis, the percent of cores involved, length of longest contiguous focus of IDP, necrosis, calcifications, biopsy-excision interval and patient age were all not significantly associated with the upgrade rate. The latter 5 variables were also not associated with rate of finding no residual papilloma in the excision. However, using a cut off point of $46.87 \%$ of core involvement by IDP (mean), the odds ratio for having residual papilloma in the excision was 3.524 ( $(\mathrm{p}=0.015)$. The mean percentage of cores involved in the "no residual group" (34.8\%) was lower than the residual group $(49.88 \%), \mathrm{p}=0.02$ (t-test)

Conclusions: In our dataset, the upgrade rate was $12.2 \%$. Most of the upgrades represent DCIS away from the biopsy site. The pathologic variables assessed did not predict an upgrade. Percent of involved cores predicts the likelihood of finding no residual papilloma in the excision at the specified cut off, but pathologically determined IDP size in the core is not associated with upgrade rate

263 Breast Cancer Biomarkers before and after Neoadjuvant Chemotherapy: Do Changes in Biomarker Status Impact Therapeutic Management?

Alexander K Quinones, Gary Tozbikian, Debra L Zynger. The Ohio State University, Columbus, $\mathrm{OH}$.

Background: In patients treated with neoadjuvant chemotherapy (NAC) there is no consensus on retesting biomarkers within the excision specimen. While previous studies have reported changes in biomarker status following NAC, corresponding changes in therapeutic management have not been investigated. Our aim is to evaluate the clinical impact of biomarker changes post-NAC at a large tertiary medical center.

Design: A retrospective search was performed to identify cases from 2013-2015 with a biopsy confirmed breast carcinoma treated with NAC and subsequent excision containing residual invasive carcinoma. Biomarkers (estrogen receptor (ER), progesterone receptor (PR) and HER2) were performed on all biopsies. Biomarker retesting was performed on the surgical specimen per pathologist preference. In cases with post-NAC repeat testing, pre- and post-NAC results were compared to identify clinically relevant changes, defined as a change from 1. ER-/PR- to ER+/PR-, 2. ER-/ PR- to ER-/PR+ and 3. HER2- to HER2+. NAC and adjuvant chemotherapy regimens in patients with a clinically relevant change were obtained via chart review.

Results: 164 NAC treated cases were identified in which $84(51.2 \%)$ had repeat testing of at least one biomarker. Clinically relevant changes were identified including 1. ER-/ PR- to ER+/PR-, 2.6\% (2/76), 2. ER-/PR- to ER-/PR+, 2.7\% (2/75) and 3. HER2- to 
HER2+ (via FISH testing), 1.3\% (1/80). 3 of the 5 changes led to modifications of the adjuvant treatment regimen. 1 ER and 1 PR change resulted in the addition of tamoxifen and the single HER2 change resulted in the addition of trastuzumab.

Conclusions: Post-NAC biomarker repeat testing in breast cancer patients reveals changes that impact therapeutic management and therefore repeat testing may be worthwhile.

264 Impact of 2013 ASCO/CAP Guidelines on HER2 Determination of Invasive Breast Cancer: A Single Institution Experience Using Frontline FISH

Moira Ragazzi, Alessandra Bisagni, Cristina Bassano, Ione Tamagnini, Elisa Gasparini, Elisabetta Kuhn, Giorgio Gardini. Arcispedale Santa Maria Nuova - IRCCS, Reggio Emilia, Italy; Azienda USL - Scandiano, Reggio Emilia, Italy.

Background: Currently, anti-HER2 targeted therapy is the therapeutic standard for HER2-positive ( + ) invasive breast cancer (IBC). HER2 test is based on either HER2 immunohistochemistry (IHC) or in situ hybridization (ISH) and interpreted based on American Society of Clinical Oncology (ASCO)/College of American Pathologists (CAP) HER2 guidelines. The 2013 ASCO/CAP guidelines expanded FISH HER2positivity, lowering the cut-off, and introduced FISH HER2-equivocal category. This study aims to evaluate the impact of 2013 ASCO/CAP HER2 guidelines in the assessment of HER2 in IBCs, including the performance of reflex testing in HER2equivocal cases.

Design: 3022 consecutive IBCs underwent frontline HER2/CEP17 FISH determination from 2009 to 2013 in our institution. All cases have been reclassified based on the 2013 ASCO/CAP guidelines. FISH HER2-equivocal cases (ratio < 2 and HER2 GCN between 4 and 6) underwent reflex tests: 1) HER2 IHC; 2) FISH using $S M S(17 \mathrm{p} 11.2)$ and 3) RARA(17q21.2) as control genes. Finally, the impact of 2013 guidelines and reflex tests were correlated with pathological characteristics.

Results: Two hundred HER2-negative cases $(6.6 \%)$ were classified differently based on the revisited guidelines. $0.3 \%(9 / 3022)$ became HER $2+$, while $6.3 \%(191 / 3022)$ became HER2-equivocal. All HER2-equivocal cases were retested with IHC and SMS/RARA FISH. Among HER2-equivocal cases, 92 cases $(48.2 \%)$ were reclassified as HER2+ using reflex tests: $14.7 \%(28 / 191)$ based on IHC, $7.9 \%(15 / 191)$ by RARA FISH and $40.8 \%$ (78/191) by SMS FISH. Moreover, 53 cases (27.7\%) resulted HER2-negative and 46 cases $(24.1 \%$ ) remained HER2-equivocal. No statistically significant differences in term of pathological features were identified between HER2-equivocal and HER2+ IBCs obtained by either one of the reflex tests.

Conclusions: Based on our study, 2013 ASCO/CAP guidelines entail: 1) HER2 classification changes in less than $7 \%$ (200/3022) of IBCs, mainly reclassifying HER2-negative cases as equivocal; 2) reflex tests together settle $76 \%(145 / 191)$ of HER2-equivocal cases; 3 ) after reflex testing, only 1.5\% (46/3022) of cases remains HER2-equivocal; 4) SMS FISH resulted the best reflex test. Further studies are warranted to evaluate the clinical benefit of those patients that become HER2+ according to 2013 guidelines, and therefore eligible for anti-HER2 therapy.

265 Integrated Radiopathologic Approach to Improve Diagnostic Accuracy of Nodal Metastasis in Breast Cancer

Deepthi Rao, Irfanullah Haider, Fang Fan, Onalisa Winblad, Ossama Tawfik. Kansas University Medical Center, Kansas City, KS.

Background: Axillary LN status is crucial for staging, treatment \& prognosis in breast cancer. Exclusion and inclusion criteria are essential for diagnostic precision and accurate prediction. Exclusion of LN metastasis can reduce unnecessary axillary surgery. Our objective is to improve the predictive accuracy of evaluating LN status by radiologists, and to correlate tumor biology on metastasis.

Design: 28 cases (14 each with positive and negative nodes) of IDC with histopathologic evaluation of LN biopsy and subsequent follow-up resection specimens were reviewed. We reviewed parameters including tumor size,tumor type/grade, ER, PR, Her-2, Ki-67 status. Radiologic criteria including LN size, cortical thickness, long \& short axis of $\mathrm{LN}$, longitudinal-transverse axis ratio (LT ratio) and LN blood flow patterns (central, peripheral, no flow or mixed) were evaluated. The diagnostic performance of radiologic criteria was correlated with tumor biology based on histopathologic variables. Results: LN metastasis correlated with tumor size (Mean $2.2 \mathrm{vs} 1.5 \mathrm{~cm}$ ), LN size (1.9 vs $1.5 \mathrm{~cm})$, long axis $(2.1 \mathrm{vs} 1.5 \mathrm{~cm})$, short axis $(1.1 \mathrm{vs} 0.69 \mathrm{~cm})$, cortical thickness ( $0.38 \mathrm{vs} 0.72 \mathrm{~mm})$; and was inversely related to LT ratio (2.31 vs 1.83$)$. No correlation was noted between LN metastasis and tumor type/grade, ER, PR, Her-2 Ki-67 status.

\begin{tabular}{|l|l|l|l|l|l|l|}
\hline & \multicolumn{2}{|c|}{ LT $<\mathbf{2}$} & \multicolumn{2}{c|}{ Cortical >4mm } & \multicolumn{2}{c|}{ LT +Cortical thickness } \\
\hline & Value (\%) & $\mathbf{9 5 \%}$ CI(\%) & Value(\%) & $\mathbf{9 5 \% ~ C I}(\%)$ & Value $(\%)$ & $\mathbf{9 5 \% ~ C I}(\%)$ \\
\hline Sensitivity & 85.7 & $57.2-98.2$ & 85.7 & $57.2-98.2$ & 71.43 & $41.9-91.6$ \\
\hline Specificity & 57.1 & $28.9-82.3$ & 71.4 & $41.9-91.6$ & 85.71 & $57.2-98.2$ \\
\hline PPV & 66.7 & $41-86.7$ & 75 & $47.6-92.7$ & 83.33 & $51.6-97.9$ \\
\hline NPV & 80 & $44.4-97.5$ & 83.3 & $51.6-97.9$ & 75 & $47.6-92.7$ \\
\hline
\end{tabular}

\begin{tabular}{|l|l|l|}
\hline & \multicolumn{2}{|l|}{ Blood flow (Central) pattern + CT $<\mathbf{4 m m}$} \\
\hline & Value (\%) & $\mathbf{9 5 \%} \mathbf{C I}(\%)$ \\
\hline Sensitivity & 92.80 & $64.1-99.6$ \\
\hline Specificity & 85.7 & $56.1-96.4$ \\
\hline PPV & 86.7 & $58.3-97.6$ \\
\hline NPV & 92.3 & $62-99.5$ \\
\hline
\end{tabular}

Table $1 \& 2$ with parameters for in ruling in \& ruling out LN metastasis.
Conclusions: More precise diagnostic parameters are needed for accurate evaluation of LN metastasis. We recommend a combined evaluation of LT axis ratio of $<2$ and cortical thickness of $>4 \mathrm{~mm}$ as quantitative indicators for enhancing the diagnostic accuracy to rule in LN metastasis. Furthermore, cortical thickness $<4 \mathrm{~mm}$ when used in conjunction with central pattern of $\mathrm{LN}$ blood flow can be immensely valuable in ruling out LN metastasis.

\section{Increasing Concordance for Intraductal Proliferative Lesions of} the Breast Using NDER, a Novel Web Application

Nicholas P Reder, Daniel Glasser, Mara H Rendi, Suzanne M Dintzis, Rochelle L Garcia, Jonathan C Henriksen, Mark R Kilgore. University of Washington Medical Center, Seattle, WA.

Background: Intraductal proliferative lesions (IPL) of the breast represent a biologic spectrum with varying risks for and progression to invasive carcinoma. Lesions associated with increased bilateral risk for invasive carcinoma include usual ductal hyperplasia (UDH) and atypical ductal hyperplasia (ADH). In contrast, lesions associated with progression to invasive carcinoma include ductal carcinoma in-situ (DCIS). Therefore, separating UDH and ADH from DCIS is of utmost importance in prognosis and treatment. Unfortunately, subtle morphologic differences can present a diagnostic dilemma in differentiating IPL. Studies have shown the highest discordance between UDH vs ADH and ADH vs low-grade DCIS. We have created a novel web application called NDER that increases inter and intra-rater reliability in the interpretation of IPL.

Design: Whole-slide images of IPL were annotated with regions of interest (ROI) and classified into five categories: no intraductal proliferation (NIP), UDH, ADH, low to intermediate grade ductal carcinoma in-situ (LGDCIS), or intermediate to high grade ductal carcinoma in-situ (HGDCIS). Then, still images measuring 1000x1000 microns were extracted from annotated ROI and used by NDER. NDER displays an image briefly (average: $4 \mathrm{~s}$ ), requires users to classify the image after the time has expired, then generates immediate feedback. An adaptive algorithm determines the time that each subsequent image is displayed, adjusting for user performance. A pilot study was conducted with 9 trainees.

Results: Users had large improvements in pre-test vs post-test performance $(53 \%$ vs $82 \%, \mathrm{p}<0.001$ ). The effect size (Cohen's $\mathrm{d}=1.92$ ) was very large, where 0.2 is a small effect, 0.5 moderate, and 0.8 large. Novice trainees experienced the greatest gains $(30 \%$ vs $75 \%$ ), while more experienced residents had lower magnitude but still significant gains $(61 \%$ vs $85 \%)$. ADH (40.5\%), UDH (52.9\%), and LGDCIS (54.5\%) were the most difficult lesions to diagnose, while NIP $(91.9 \%)$ and HGDCIS $(70.9 \%)$ had the highest accuracy. On average, the module lasted 30 minutes.

Conclusions: NDER is a novel web app that rapidly increases accuracy and evaluates for diagnostic proficiency. ADH and UDH remained difficult lesions to classify, however, there was significant diagnostic improvement from pre-test to post-test performance. NDER is an efficient, promising method to increase concordance for challenging IPL. NDER can be used not only for training, but also for quality assurance amongst established pathologists. Further studies with larger cohorts are necessary.

267 Negative Progesterone Receptor Status, Mitotic Activity and Periductal Chronic Inflammation (PDCI) Around DCIS Does Not Predict Recurrences in DCIS Cases

Bailey A Reindl, Shabnam Jaffer, Ira J Bleiweiss, Anupma Nayak. Mount Sinai Hospital, New York, NY.

Background: Risk factors predictive for local recurrence of DCIS or progression to invasive ductal carcinoma (IDC) are not well established. Recent studies have suggested that negative progesterone receptor status, mitotic activity and dense periductal chronic inflammation (PDCI) around DCIS may predict recurrence of DCIS and thus serve as histologic surrogates for the Oncotype DX DCIS recurrence score. The purpose of this study was to validate these results in a retrospective cohort of DCIS with recurrences. Design: Using our pathology database we retrospectively identified 33 patients (1996 - 2015) with an initial diagnosis of DCIS and subsequent local recurrence of DCIS or progression to IDC. Another 33 patients $(2004-2005)$ with DCIS without any recurrences (matched with age and histologic grade) served as control group. Clinicopathologic data was recorded from electronic medical records. The H\&E stained slides from the study and control groups were reviewed to record the histologic features. Results of the two groups were compared using statistical analysis.

Results: Of the 33 patients in the study group (age range of 34 to 83 years; median 62 years), 1 had low grade DCIS, 14 intermediate grade DCIS and 18 high grade DCIS. Twenty three patients had lumpectomy with or without radiation and 10 had mastectomy. None of the patients had positive margin status at the end of primary surgical treatment. Nineteen patients had local recurrence of DCIS and 14 had progression to IDC at median interval of 34 months (range, $6-136$ months). The control group without recurrences (age, 42 to 78 years; median 57 years) was comprised of 1 low grade DCIS, 14 intermediate grade DCIS and 18 high grade DCIS with median follow up of 86 months (range, $64-125$ months). The comparison of histologic parameters in the study versus control group is as follows; mitotic count ( 0 to $27 / 10 \mathrm{hpf}$ vs. 0 to $24 / 10 \mathrm{hpf}$; $\mathrm{p}=0.52)$, presence of necrosis ( $23 \mathrm{vs} .26 ; \mathrm{p}=0.57)$, comedo-necrosis $(10 \mathrm{vs} .12 ; \mathrm{p}=0.79)$, calcifications ( 26 vs. $24 ; \mathrm{p}=0.77)$, PDCI ( 5 vs. $9 ; \mathrm{p}=0.37)$, lobular cancerization (12 vs. $7 ; \mathrm{p}=0.28$ ), estrogen receptor status ( 26 vs. $28 ; \mathrm{p}=0.75$ ), progesterone receptor status ( 18 vs. $25 ; \mathrm{p}=0.12$ ).

Conclusions: None of the histologic features including, negative progesterone receptor status, mitotic count and dense periductal chronic inflammation (PDCI) around DCIS were present in statistically significant proportion in DCIS patients with recurrences versus those without. Future studies to discover new biologic markers for risk stratification of DCIS cases are needed. 
268 TERT Hotspot Promoter Mutations and TERT Gene Amplification in Phyllodes Tumors of the Breast

Jorge S Reis-Filho, Salvatore Piscuoglio, Charlotte KY Ng, Melissa Murray, Kathleen Burke, Marcia Edelweiss, Gabriel S Macedo, Felipe C Geyer, Akiko Inagaki, Anastasios D Papanastasiou, Luciano G Martelotto, Caterina Marchio, Raymond Lim, Ino De Bruijn, Lilian Smyth, Muzaffar Akram, Dara S Ross, Larry Norton, David B Solit, Jose Baselga, Edi Brogi, Marc Ladanyi, Britta Weigelt. Memorial Sloan Kettering Cancer Center, New York, NY.

Background: Phyllodes tumors (PTs) of the breast are fibroepithelial tumors, whose clinical behavior varies according to histologic grade (i.e. benign, borderline or malignant PTs). The distinction between borderline and malignant PTs and between benign PTs and fibroadenomas (FAs) is challenging. Recent studies have reported recurrent MED12 exon 2somatic mutations in FAs and PTs, suggesting that these lesions are likely closely related. We sought to define the repertoire of somatic genetic alterations in PTs and whether these may assist in the correct diagnosis of these lesions. Design: We collected 100 FAs, 40 benign PTs, 14 borderline PTs and 22 malignant PTs. Six benign, 6 borderline and 13 malignant PTs and matched normal tissue were subjected to targeted massively parallel sequencing (MPS) using the MSK-IMPACT assay. MED12 and TERT mutations were investigated in all samples using a combination of Sanger sequencing and amplicon sequencing. TERT mRNA was assessed by qRTPCR and and TERT gene copy number was investigated by quantitative PCR (qPCR). Results: MSK-IMPACT sequencing confirmed the presence of MED12 exon 2 mutations in a subset of PTs, and revealed clonal somatic mutations in key cancer genes (e.g. TP53, $R B 1, S E T D 2$ and EGFR) exclusively in malignant PTs. We identified a recurrent clonal hotspot mutation in the TERT promoter (C228T) in 52\% and TERT amplification in $4 \%$ of PTs. Assessment of MED 12 mutations and TERT genetic alterations in the entire cohort by Sanger sequencing, amplicon sequencing and/or qPCR analysis demonstrated that $M E D 12$ exon 2 mutations were more frequent in benign $(80 \%)$ and borderline PTs $(64 \%)$ than in malignant PTs $(23 \% ; P<0.01)$. Conversely, the frequency of TERT alterations increased from benign ( $18 \%)$ to borderline $(57 \%)$ and malignant PTs $(68 \%$; $P<0.01)$. TERT mRNA levels were significantly higher in PTs harboring altered TERT than in TERT wild-type PTs $(\mathrm{P}<0.001)$. In FAs, no TERT alterations were detected. The presence of a TERT C228T promoter mutation sequencing and/or TERT gene amplification distinguished PTs from FAs with a sensitivity and a positive predictive value of $100 \%$ and $100 \%$, respectively.

Conclusions: The TERT C228T promoter mutation and/or TERT gene amplification likely play a role in the progression of PTs, and their analysis may constitute an ancillary diagnostic tool in the differentiation of PTs from FAs.

269 Prediction of Breast Cancer Recurrence Risk Categories Using Automatic Mitotic Detection Algorithms Based on Deep Learning

David Romo-Bucheli, Andrew Janowczyk, Hannah Gilmore, Eduardo Romero, Anant Madabhushi. Universidad Nacional de Colombia, Bogota, Colombia; University Hospitals, Cleveland, OH; Case Western Reserve University, Cleveland, $\mathrm{OH}$

Background: The 21 gene expression Oncotype DX (ODX) test provides a recurrence risk score for identifying early stage estrogen receptor positive (ER+) breast cancers (Bca) that require adjuvant chemotherapy. Recent work from a few groups suggests that computational image analytic approaches on routine digitized Hematoxylin and Eosin (H\&E) pathology images could also be used to assess Bca risk. In this work, we used a Deep Learning (DL) algorithm to identify mitoses in Whole Slide Images (WSI) and- then evaluated the association between the automatic mitotic count with ODX risk (low vs high) categories.

Design: Nuclei image patches were extracted from two open access datasets (MITOS2012 and AMIDA2013) for training the DL model to detect mitoses on H\&E images. Then, the DL model was used on 40X High Power Fields (HPF) of 54 WSI of early stage ER+ breast cancers. The 54 cases comprised (a) a low risk group of 39 cases $($ ODX score $<18)$ and low modified bloom richardson $(\mathrm{mBR})$ grade $(<6)$ and $(\mathrm{b})$ a high risk group of 15 cases with a ODX score $>30$ and $\mathrm{mBr}$ grade $>7$. The mitotic count per HPF in each WSI was used to train a Support Vector Machine (SVM) classifier to distinguish between high and low risk WSI cases. Classifier training and testing was done using a leave one out scheme.

Results: The automatically computed mean mitotic count per $10 \mathrm{HPF}$ was found to be significantly different ( $\mathrm{p}$-value $<0.01$ ) between the risk groups (See Figure 1), suggesting that mitotic density is useful in discriminating low and high risk $\mathrm{BCa}$. Additionally, the SVM classifier trained with mitotic count frequency yielded an $87 \%$ accuracy (See confusion matrix in Figure 1).
Distribution of mitotic Figure (10HPF)

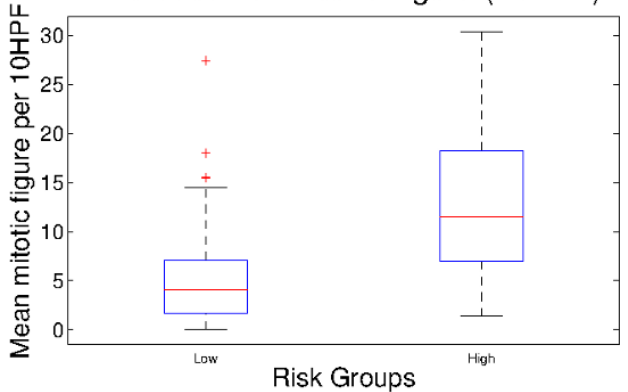

\begin{tabular}{|l|l|l|}
\hline $\begin{array}{l}\text { Estimated Risk } \\
\text { Category }\end{array}$ & $\begin{array}{l}\text { Low ODX and } \\
\text { mBR score }\end{array}$ & $\begin{array}{l}\text { High ODX and } \\
\text { mBR score }\end{array}$ \\
\hline Low Risk & 36 & 3 \\
\hline High Risk & 4 & 11 \\
\hline
\end{tabular}

Figure 1: Mean mitotic figure per $10 \mathrm{HPF}$ for the low and high risk groups. In the table, the confusion matrix for risk category classification using automatic mitotic count. Conclusions: In our work we identified a correlation of mitotic count, as determined by a deep learning algorithm, with the low and high ODX risk categories. Additionally, a SVM classifier using mitotic count information was able to distinguish low and high ODX risk categories.

270 Analysis of Estrogen Receptor (ER) Immunohistochemistry (IHC) in Breast Cancer with ESR1 Mutations Detected by Hybrid Capture-Based Next Generation Sequencing (NGS)

Dara S Ross, Fumiko Konno, Edi Brogi, Ahmet Zehir, Michael F Berger, Sarat Chandarlapaty, Pedram Razavi, Jose Baselga, Hannah $Y$ Wen. Memorial Sloan Kettering Cancer Center, New York, NY.

Background: ER- $\alpha$, encoded by ESR1, is expressed in $\sim 70 \%$ of invasive breast cancers (BC). ESR1 mutations involving the ligand binding domain (LBD) (aa 302 to 595) are reported in ER-positive BC after prolonged endocrine therapy (ET) (Toy et al. Nat Genet. 2013) and some confer constitutive ligand-independent ER activity, mediating resistance.

Design: The MSK-IMPACT (Integrated Mutation Profiling of Actionable Cancer Targets) assay utilizes solution-phase exon capture and NGS to detect somatic mutations in the most common cancer related genes ( 410 genes in current platform). BC from patients (pts) with locally advanced or metastatic disease that underwent MSK-IMPACT testing between 1/2014 to 5/2015 and yielded ESR 1 mutations were identified. Clinical information was retrieved from the medical records. Three pathologists reviewed all available slides.

Results: A total of $586 \mathrm{BC}$ were analyzed and $67 \mathrm{BC}$ ( 57 post ET metastases, 5 post ET primary BC, 5 untreated primary $\mathrm{BC}$ ) from 66 pts had one or more ESR1 mutation. Median age at primary BC diagnosis was 48 years (range 29-81). The primary BC were ductal (43), lobular (13), and mixed ductal and lobular (10). Median primary tumor size available for $35 / 66$ pts was $2 \mathrm{~cm}$. Receptor status was available for 53/67 BC: 53 ER-positive, 40 PR-positive, 2 HER2-positive. ESR1 mutations included 60 single nucleotide alterations (SNA) and 11 copy number alterations (10 gains, 1 loss). Twenty unique SNA were identified, all involving the LBD. The most frequent SNA were p.D538G (22), p.Y537S (7) and p.E380Q (7). Forty SNA affected amino acids L536 to D538 (known activating mutations), and all were identified in post ET BC. The assay also uncovered concurrent mutations in 28 cancer genes, most common including PIK3CA, GATA3, CDH1 and TP53. ER IHC was available in the paired primary and metastatic BC for 29 pts and no change was observed in the ER status. Substantially decreased ER positivity was seen in 3 decalcified specimens. PR IHC was available for 28 of these 29 pts and 3 cases showed substantially decreased PR positivity in the metastases.

Conclusions: We identified ESR I LBD SNA in 10\%(60/586) of BC analyzed. The most common SNA within the LBD were p.D538G, p.Y537S and p.E380Q, which together accounted for $51 \%(36 / 71)$ of all ESR1 mutations. Our results show that ER status by IHC does not change during disease progression, even in cases with ESRI mutations.

271 MCL1 Is a Predictive Biomarker of Triple Negative Status and Therapy Response in Relapsed and Metastatic Breast Cancer

Jeffrey SRoss, Sungeun Kim, Aseeb Rehman, Ann B Boguniewicz, Christine E Sheehan, Kai Wang, Adrienne Johnson, Julia A Elvin, Jo-Anne Vergilio, James Suh, Vincent A Miller, Philip J Stephens, Siraj M Ali. Albany Medical College, Albany, NY; Foundation Medicine Inc., Cambridge, MA.

Background: $M C L 1$ (myeloid leukemia cell differentiation protein Mcl-1) is a member of the $B C L-2$ family and functions to inhibit apoptosis. To date, the role of $M C L 1$ genomic alterations (GA) and MCL1 portein overexpression for the prediction of therapy response in breast cancer (BC) has not been widely studied.

Design: Comprehensive genomic profiling (CGP) using hybridization capture of 3,769 exons from 315 cancer-related genes and 47 introns of 19 genes commonly rearranged in cancer was applied to $\geq 50$ ng of DNA extracted from 2,824 consecutive BC and sequenced to high, uniform median coverage $(>600 \mathrm{X})$. All classes of genomic alterations 
including substitutions, insertions, deletions, fusions and compy number alterations were assesed. A series of $78 \mathrm{BC}$ were immunostained (IHC) for MCL1 expression (clone SC-819, Santa Cruz). Results were correlated with clinical outcome.

Results: The 200 patients whose BC underwent CGP had a median age of 55 years (range 27-91 years). Of the $200(7.1 \%)$ BC with $M C L 1$ amplification, the $146(73 \%)$ enrichment of MCL1 amplification in triple negative (TN) vs non-TN was highly significant $(\mathrm{p}<0.0001)$. Of the $M C L 1$ amplified TNBC cases $88 \%$ were high grade and $98 \%$ were stage IV at the time of sequencing. The original primary $\mathrm{BC}$ was assayed in $44 \%$ of cases and a sample from a metastatic focus in $56 \%$ of cases. Of the $200 \mathrm{MCL} 1$ amplified BC, 12 (6\%) were ERBB2 (HER2) amplified and slide-based HER2 status (IHC and/or FISH) concordance with GCP was $99 \%$. Genes co-altered with in $M C L 1$ amplified TNBC included TP53 (86\%), MYC (41\%), MYST3 (21\%), LYN (20\%), CCNE1 (19\%), PIK3CA (18\%), and AKT3 (15\%). In the 78 patient IHC study, MCL1 overexpression correlated with TNBC and PR- status but was not an independent predictor of clinical outcome in a multivariate model. Two MCL1 amplified TNBC patients were treated with a multi-drug regimen based on sorafenib and vorinostat and showed significant sustained tumor responses in highly pre-treated patients.

Conclusions: $M C L 1$ amplification is a frequent feature in advanced stage and high grade TNBC. MCL1 amplified BC are very seldom ERBB2 co-amplified. Clinical observation suggests that treatment with sorafenib and vorinostat in heavily pre-treated MCL1 amplified BC may be correlated with clinical benefit, consistent with previous preclinical investigations. These preliminary findings suggest that MCL1 amplified TNBC may be able to benefit from combination targeted therapy, and warrant further investigation in a prospective clinical trial.

272 The Expression of Metallopanstimulin-1 in Low and High-Grade In Situ and Invasive Breast Ductal Carcinoma

Daryoush Saeed-Vafa, Marilin Rosa. Moffitt Cancer Center and Research Institute, Tampa, FL.

Background: Previous studies have demonstrated increased serum and tissue expression of metallopanstimulin-1 (MPS-1), a ribosomal subprotein 27 (RPS27) in cases of breast cancer, as well as in other cancers, such as prostate, colon, gastric, and melanoma. It has also been found that MPS-1 was present at low levels in benign breast tissue but was overexpressed in invasive breast carcinomas and that the expression of MPS- 1 was greater in breast tumors with greater size and histological grade. To our knowledge, this difference has yet to be quantified using an immunofluorescence (IF) assay. Furthermore, there is little in the literature exploring the expression of MPS-1 in breast low and high-grade in situ (DCIS) and invasive ductal carcinomas (IDC). In this pilot study we attempted to quantify the level of MPS-1 in a subset of cases to evaluate the differences in expression among low and high-grade breast carcinomas.

Design: A total of 17 cases, 6 high-grade DCIS, 3 low-grade DCIS, 4 high-grade IDC, and 4 low-grade IDC, were selected from the Moffitt Cancer Center. MPS-1 expression was studied via an IF assay on whole tissue section slides using a monoclonal MPS-1 antibody (1:1000), clone [N1], with a TSA amplification kit along with a $\mathrm{pH} 9$ antigen retrieval solution in a pressure cooker for 3 mins $\left(121 \mathrm{psi} / 121^{\circ} \mathrm{C}\right)$. MPS-1 expression was quantified via an Automated Quantitative Software Analysis (AQUA) system targeting tumor cells.

Results: Our analysis revealed that MPS-1 was more strongly expressed in highgrade DCIS as compared to low-grade DCIS, mean AQUA scores of 111.06 and 50.86 respectively. This same pattern was observed when comparing high-grade IDC as compared to that of low-grade IDC, mean AQUA scores of 239.86 and 62.63 respectively.

Conclusions: The distribution pattern of MPS-1 in DCIS and IDC demonstrates that the MPS-1 protein is overexpressed in areas of active proliferation. The observation that both low-grade DCIS and low-grade IDC have lower expression of MPS-1 as compared to that of high-grade DCIS and high-grade IDC follows previously published studies that low-grade proliferations, both in situ and invasive, are more probably related while highgrade proliferations are believed to involve a separate pathway. Furthermore, our data provides further evidence that the MPS-1 protein may play an important role in cancer cell proliferation and in many neoplasias. Future studies evaluating the expression of MPS-1 in the spectrum of benign, borderline and malignant breast lesions are necessary to understand its involvement in breast tumor progression.

\section{Impact of Cavity Shave Margins in Lumpectomies for Breast} Carcinoma on Final Margin Status and Rate of Re-excision

Nagla Salem, Sudeshna Bandyopadhyay, Vishakha Pardeshi, MHD Fayez Daaboul, Lydia Choi-Kim, Rouba Ali-Fehmi, Shashi Madan. WSU/DMC, Detroit, MI.

Background: The advantage of lumpectomies with cavity shave margins (CSM) for breast carcinoma, (margins submitted separately) versus lumpectomies without CSM is not well established. This is a comparison of the two procedures' impact on margin status and rate of re-excision.

Design: We evaluated 549 cases of ductal carcinoma in situ (DCIS) and/or invasive ductal carcinoma (IC) in the period from June 2013 to June 2015, of which 142 underwent lumpectomies with CSM and 407 underwent lumpectomies without CSM. The following were analyzed for each procedure: positive margins for DCIS or IC, presence of DCIS or IC within $2 \mathrm{~mm}$ from the margin and the rate of re-excision. H \& E slides of positive margins (tumor on ink) were reviewed by 2 pathologists.

Results: Among patients (pts) who underwent lumpectomies without CSM, 306 pts had DCIS and 302 had IC, while 89 had DCIS and 112 had IC in those who underwent lumpectomies with CSM. Less pts had IC at the margin after lumpectomies with CSM compared to lumpectomies without CSM $(4.4 \% \& 10.4 \% ; \mathrm{p}=0.077)$ respectively. More pts had DCIS at the margin after lumpectomies with CSM compared to lumpectomies without CSM $(13 \% \& 10 \%$; $=0.443)$ respectively. Less pts had IC within $2 \mathrm{~mm}$ from the margin after lumpectomies with CSM compared to lumpectomies without CSM $(20.5 \%$
$\& 27.7 \%$; $=0.16$ ), respectively. Less pts had DCIS within $2 \mathrm{~mm}$ from the margin after lumpectomies with CSM compared to lumpectomies without CSM $(32.6 \% \& 41.2 \%$; $\mathrm{p}=0.15)$, respectively. The rate of re-excision was higher in pts who underwent lumpectomies without CSM compared to pts who underwent lumpectomies with CSM $(24.1 \% \& 7.7 \% ; \mathrm{p}=0.001)$, respectively. Table 1 : Comparison of the 2 procedures.

\begin{tabular}{|l|l|l|l|}
\hline & $\begin{array}{l}\text { Lumpectomies with } \\
\text { CSM }\end{array}$ & $\begin{array}{l}\text { Lumpectomies witout } \\
\text { CSM }\end{array}$ & $\begin{array}{c}\text { p } \\
\text { value }\end{array}$ \\
\hline IC at margin & & & \\
\hline Yes & $5 / 113(4.4 \%)$ & $31 / 299(10.4 \%)$ & \\
\hline No & $108 / 113(95.6 \%)$ & $268 / 299(89.6 \%)$ & 0.077 \\
\hline DCIS at margin & & & \\
\hline Yes & $12 / 92(13 \%)$ & $31 / 309(10 \%)$ & \\
\hline No & $80 / 92(87 \%)$ & $278 / 309(90 \%)$ & 0.443 \\
\hline $\begin{array}{l}\text { IC within 2mm from } \\
\text { margin }\end{array}$ & & & \\
\hline Present & $23 / 112(20.5 \%)$ & $83 / 300(27.7 \%)$ & \\
\hline Absent & $89 / 112(79.5 \%)$ & $217 / 300(72.3 \%)$ & 0.16 \\
\hline $\begin{array}{l}\text { DCIS within 2mm from } \\
\text { margin }\end{array}$ & & & \\
\hline Present & $31 / 95(32.6 \%)$ & $127 / 308(41.2 \%)$ & \\
\hline Absent & $64 / 95(67.4 \%)$ & $181 / 308(58.8 \%)$ & 0.15 \\
\hline Re-excision & & & \\
\hline Done & $11 / 142(7.7 \%)$ & $98 / 407(24.1 \%)$ & \\
\hline Not done & $131 / 142(92.3 \%)$ & $309 / 407(75.9 \%)$ & 0.001 \\
\hline
\end{tabular}

Conclusions: Lumpectomies with CSM reduced positive margins for IC as well as IC and DCIS within $2 \mathrm{~mm}$ from the margin and the rate of re-excision.

\section{Histologic Grade 1 in Breast Cancer Indicates a Lack of Benefit in} Ordering Oncotype DX

Mary Ann Sanders, Elizabeth C Riley. University of Louisville, Louisville, KY.

Background: Oncotype DX is a 21 gene RT-PCR assay that assists in the decision to administer chemotherapy for estrogen receptor (ER) positive, HER2 negative invasive breast cancer (BC). Since most genes analyzed by Oncotype DX relate to cell proliferation, ER and HER2, predicting Oncotype DX Recurrence Score (RS) with routine histopathology has been the focus of many studies likely motivated by Oncotype DX cost and delay in treatment while awaiting results. Oncotype DX is included in the NCCN guidelines for ER positive HER2 negative invasive BC $>5 \mathrm{~mm}$. Such minimal criteria could encourage ordering this assay without regard to additional relevant pathologic characteristics. The purpose of this study was to identify pathologic parameters routinely provided in pathology reports that can determine the necessity of ordering Oncotype DX.

Design: This is a retrospective review of pathology reports from cases of ER positive, HER2 negative invasive BC with Oncotype DX RS results from our database from 2006 to 2015. Clinicopathologic data recorded include histologic grade, ER, progesterone receptor $(\mathrm{PR})$, and $\mathrm{Ki}-67$. Oncotype $\mathrm{DX} \mathrm{RS}$ was recorded as low risk (LR) $(\mathrm{RS}=$ $0-17)$, intermediate risk $(\mathrm{IR})(\mathrm{RS}=18-30)$ and high risk $(\mathrm{HR})(\mathrm{RS}=31-100)$. IR was subcategorized into low-intermediate $(\mathrm{L}-\mathrm{I})(\mathrm{RS}=18-25)$ and intermediate-high $(\mathrm{RS}=$ $26-30$ ) risk, cutoffs used in the Oncotype DX TAILORx study (ClinicalTrials.gov). Results: We identified 133 cases: 80 were LR, 39 were IR and 14 were HR. In cases with available PR percent staining, PR $\leq 33 \%$ occurred in 3 of 74 (4\%) LR, 15 of 39 (39\%) IR, and 8 of $14(57 \%)$ HR cases. In cases with available Ki-67, a result of $\geq 20 \%$ occurred in 12 of $52(23 \%)$ LR, 10 of $20(50 \%)$ IR, and 9 of $10(90 \%)$ HR cases. Thirtyone cases were grade 3: $10(32 \%)$ were LR, $9(29 \%)$ were IR and $12(39 \%)$ were HR. Seventy-nine cases were grade 2: 43 (54\%) were LR, $34(43 \%)$ were IR and $2(3 \%)$ were HR. Twenty-three cases were grade 1: $20(87 \%)$ were LR, $3(13 \%)$ were IR, and $0(0 \%)$ were HR. The 3 IR grade 1 cases were subcategorized to L-I risk. All grade 1 tumors were ER positive (mean 94\%) and PR positive (mean 84\%), and Ki-67 ranged from $3-35 \%$ (mean $12 \%)$.

Conclusions: All grade 1 cases were LR or L - I risk subcategory by Oncotype DX; results that would not impact treatment decisions for these patients. Our data shows the value of grade 1 histology as a reliable indicator for the lack of benefit in ordering Oncotype DX which would lead to significant cost savings and avoid treatment delay in this subset.

275 PD-L1 Expression and Lymphocytic Response in NeoadjuvantTreated Breast Cancers

Nina Schatz-Siemers, Beth Harrison, Silvia C Formenti, Sandra Demaria. NYU Langone Medical Center, New York, NY; Weill Cornell Medical College, New York, NY. Background: Programmed Death Ligand-1 (PD-L1) is a transmembrane immune regulatory molecule that inhibits cytotoxic T-lymphocyte (CTL) activity. PD-L1 expression on cancer cells and tumor-infiltrating myeloid cells is thought to be a major mechanism of tumor immune escape and to predict response to antibodies targeting the PD-1/PDL-1 pathway. Previous studies have shown that high PD-L1 expression in breast tumors was associated with tumor-infiltrating lymphocytes (TILs) and predicted pathologic complete response ( $\mathrm{pCR}$ ) to neoadjuvant chemotherapy. The objective of this study was to evaluate PD-L1 expression and TILs in the residual tumor of neoadjuvanttreated patients with pathologic partial response (pPR).

Design: 26 cases, including 21 with pPR and 5 with pCR, were selected from a cohort of patients with locally advanced breast cancer and treated with neoadjuvant radiation \& chemotherapy (Adams et al., Breast Cancer Res Treat, 2010). Pre-treatment biopsies 
were available from 19 patients for evaluation of TIL percentage on H\&E-stained slides, and 13 had sufficient tissue for marker analysis. Post-treatment excision of the 21 patients with $\mathrm{pPR}$ and pre-treatment biopsies were evaluated for PD-L1 expression, assessed by staining with SP-142 antibody (Spring Bioscience) and for percentages of CTLs and regulatory $\mathrm{T}$ cells (Tregs) assessed by staining for $\mathrm{CD} 8$ and FoxP3, respectively. Results: PD-L1 was considered positive if intensity of membrane staining was at least $2+$ (on a scale of 0 to $3+$ ) and at least $1 \%$ of the tumor cells were positive. $3 / 13$ pretreatment cases were PDL-1+. Two had TILs $>60 \%$, consistent with the definition of LPBC (Loi et al., J Clin Oncol, 2013). One of them was a pCR. The other showed pPR with scattered small nests of residual tumor cells showing reduced PDL-1 expression $(1+$, negative $)$ and an increase in CD8/Treg ratio. Pre-treatment, the third PDL-1+ case had $20 \%$ TILs. Post-treatment, it showed increased PDL-1 expression (20\% to $50 \%$ of the tumor cells) and a marked increase in CD8/Treg ratio. The only other patient showing PDL-1+ post-treatment did not have pre-treatment tissue available for evaluation. It showed $30 \%$ TILs post-treatment with high CD8/Treg ratio.

Conclusions: Data show that PD-L1 is expressed in a subset of tumors post-treatment with radiation and chemotherapy. In this small cohort, the association of PDL-1 positivity with increase in $\mathrm{CD} 8 /$ Treg ratio suggests that at least in some patients anti-tumor $\mathrm{T}$ cells may contribute to tumor elimination, which may be hindered by PDL-1 expression.

276 Sentinel Lymph Node Status and Patterns of Invasion in Microinvasive Breast Carcinoma

Christopher Sebastiano, Saipriya Iyer, Anibal Cordero, George Plitas, Dilip Giri. Memorial Sloan Kettering Cancer Center, NY, NY.

Background: Microinvasive breast carcinoma (MIC) infrequently metastasizes to axillary lymph nodes. We investigated the clinicopathologic features associated with lymph node status, as well as morphologic patterns of microinvasive disease.

Design: A database from our institution of 426 patients diagnosed with MIC was searched for patients for whom histologic material containing microinvasion (core biopsy, excision/lumpectomy, or mastectomy) was available for review. 19 patients were excluded on review due to absence of definite microinvasion or reclassification as invasive carcinoma. Clinical data were obtained, and available histologic material was reviewed.

Results: A total of 238 patients were identified, all of whom were female. Patients ranged in age from 27 to 83 at time of presentation (median age 53). 9 patients (4\%) had lobular carcinoma in situ (LCIS) as the associated in situ component. The remainder had ductal carcinoma in situ (DCIS), the majority of which $(154 ; 67 \%)$ was of high nuclear grade. 3 major morphologic patterns of microinvasion were identified and their relative frequency calculated (patterns were not mutually exclusive): clusters $(78 \%)$, single cells (67\%), and gland forming (5\%); (Figure 1). A total of 24 patients (10\%) had sentinel lymph node metastases; 16 (66.7\%) isolated tumor cells (ITC) only, $5(20.8 \%)$ micrometastases, and $3(12.5 \%)$ macrometastases (2 to $3 \mathrm{~mm}$ ). Clinicopathologic parameters (age, presence and size of a mass forming lesion, grade of DCIS, extent of in situ carcinoma, number of foci of microinvasion, morphologic patterns of microinvasion, presence of lymphovascular invasion, and ER/Her2 biomarker status) were compared between patients with and without sentinel lymph node metastases. Only extent of in situ carcinoma (as quantified by the number of slides involved out of the total slides evaluated) was significantly associated with sentinel node status $(p=0.03)$.

Conclusions: The most common morphologic patterns of MIC are clusters and single cell invasion. Sentinel node metastases are uncommon in association with MIC, especially micro- or macrometastases. Extent of in situ carcinoma is the sole clinicopathologic feature associated with sentinel lymph node status.

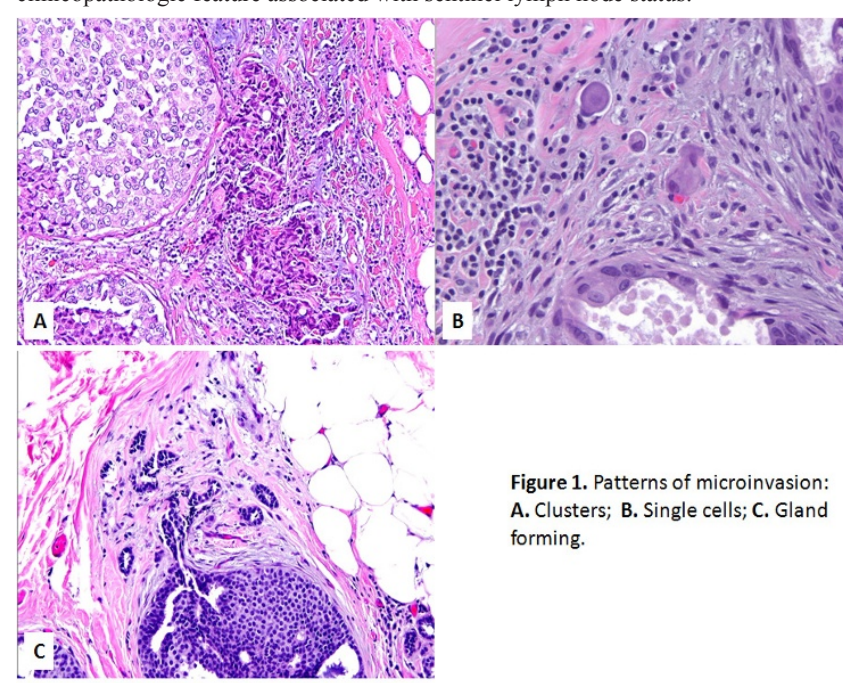

277 Benign Vascular Lesions of the Breast Do Not Require Excision Christopher Sebastiano, Lucas Gennaro, Edi Brogi, Elizabeth Morris, Muzaffar Akram, Zenica L Bowser, Adriana D Corben, Sandra Brennan, Melissa Murray. Memorial Sloan Kettering Cancer Center, NY, NY.

Background: Current guidelines recommend excision of all vascular lesions (VL) of the breast identified by core needle biopsy (CBX). We investigated whether excision of non-atypical VL is required.
Design: We searched the electronic medical record for patients with VL of the breast diagnosed on CBX between 2000 - 2015. Clinical data were obtained, and all available radiologic and histologic material was reviewed.

Results: A total of 94 patients ( 93 female, 1 male) with median age of 50 years (range 12 - 93) had index CBX diagnosis of VL, including 3 angiosarcomas (AS), 5 atypical vascular lesions (AVL), and 86 benign vascular lesions (BVL). The BVL consisted of 83 hemangiomas, 2 angiolipomas, and 1 papillary endothelial hyperplasia. A radiologist reviewed all pre- and post-CBX imaging studies; all cases had concordant radiologic and pathologic findings. VL was the radiologic target in 44 (47\%) cases (Table 1). 50/86 (58\%) BVL were deemed incidental findings (median size $1 \mathrm{~mm}$ microscopically). Follow up excision was performed on 22/94 (23\%) VL, including 14/44 (32\%) targeted VL. There were no diagnostic upgrades at excision. Follow up excision of 8 incidental BVL yielded 2 residual hemangiomas and 6 cases with no residual lesion.

Conclusions: Surgical excision of malignant and atypical vascular lesions is required. BVL showed no upgrades at excision. No recurrences or clinical events were observed in patients diagnosed with BVL with a median followup of 24.5 months. Our data suggest that benign vascular lesions identified in $\mathrm{CBX}$ with concordant radiologic and pathologic findings do not require surgical excision.

\begin{tabular}{|l|l|l|l|}
\hline \multicolumn{1}{|c|}{ Table 1. Targeted Vascular Lesions } \\
\hline CBX Diagnosis & $3 \mathrm{AS}$ & $5 \mathrm{AVL}$ & $36 \mathrm{BVL}$ \\
\hline $\begin{array}{l}\text { Median Imaging Size } \\
\text { (mm) }\end{array}$ & 38.3 & 11.3 & 8.4 \\
\hline CBX Modality & $\begin{array}{l}3 \\
\text { Ultrasound }\end{array}$ & $\begin{array}{l}\text { 2 Ultrasound } \\
\text { 2 Stereotactic } \\
1 \text { MRI }\end{array}$ & $\begin{array}{l}18 \text { Ultrasound } \\
7 \text { Stereotactic } \\
11 \text { MRI }\end{array}$ \\
\hline Excision Diagnosis & $3 \mathrm{AS}$ & $\begin{array}{l}\text { 2 Hemangioma } \\
\text { 2 Angiolipoma } \\
1 \text { No residual }\end{array}$ & $\begin{array}{l}\text { 2 Hemangioma } \\
\text { 4 No residual }\end{array}$ \\
\hline $\begin{array}{l}\text { Radiologic Followup If } \\
\text { Not Excised }\end{array}$ & 0 & 0 & $\begin{array}{l}\text { 9 No residual on post-CBX } \\
\text { imaging } \\
14 \text { Stable on post-CBX imaging } \\
7 \text { No followup imaging }\end{array}$ \\
\hline $\begin{array}{l}\text { Median Followup Time } \\
\text { (Months) }\end{array}$ & 14 & 15.8 & 24.5 \\
\hline $\begin{array}{l}\text { Clinical Followup } \\
\text { 1 NED } \\
1 \text { DOD } \\
1 \text { LTFU }\end{array}$ & $\begin{array}{l}3 \text { NED } \\
\text { 2 LTFU }\end{array}$ & $\begin{array}{l}\text { 24 NED } \\
\text { 2 DOC } \\
10 \text { LTFU }\end{array}$ \\
\hline $\begin{array}{l}\text { DOD - died of disease, DOC - died of other causes, LTFU - lost to followup, NED - no evidence } \\
\text { of disease }\end{array}$
\end{tabular}

\section{Male Breast Cancers: Are They Different Than Female Breast} Cancers?

Kate M Serdy, David Dabbs, Rohit Bhargava. Magee-Womens Hospital of University of Pittsburgh Medical Center, Pittsburgh, PA.

Background: Invasive breast carcinoma is expected to affect over 2000 men in the United States in 2015. We performed a detailed clinical-pathologic study of male breast carcinoma to determine if any clinical and morphological differences exist between males and females.

Design: Sixty-one cases of male invasive breast cancer were identified in ten year period at our institution (2003-2013). Slides were reviewed for tumor grading. Treatment and clinical follow up was obtained from tumor registry. The pathologic variables of male breast cancers were compared with a pre-existing dataset of 198 consecutive female breast cancers.

Results:

\begin{tabular}{|l|l|l|l|}
\hline & Male & Female & P value \\
\hline Mean Age in Years & 64 & 60 & $0.0202^{*}$ \\
\hline Mean Tumor Size $(\mathrm{cm})$ & 2.3 & 1.7 & $0.0002^{*}$ \\
\hline No. Ductal Cancers & $60 / 61(98 \%)$ & $181 / 198(91 \%)$ & 0.0821 \\
\hline Nottingham Score $\geq 7$ & $39 / 61(64 \%)$ & $93 / 198(47 \%)$ & $0.0276^{*}$ \\
\hline Lymph Node + & $29 / 55(53 \%)$ & $62 / 182(34 \%)$ & $0.0173 *$ \\
\hline ER + & $58 / 60(95 \%)$ & $164 / 198(83 \%)$ & $0.0196 *$ \\
\hline PR + & $54 / 60(90 \%)$ & $160 / 198(81 \%)$ & 0.1178 \\
\hline Mean ER H-score & 240 & 228 & 0.2212 \\
\hline Mean PR H-score & 150 & 130 & 0.1803 \\
\hline HER2 + & $6 / 60(10 \%)$ & $26 / 198(13 \%)$ & 0.6566 \\
\hline Mean Ki-67 Index & $33 \%($ based on 56 cases) & $25 \%($ based on 192 cases) & $0.0145 *$ \\
\hline Number of Stage I Cases & $12 / 55(22 \%)$ & $119 / 198(60 \%)$ & $<0.0001 *$ \\
\hline *Statistically significant & & & \\
\hline
\end{tabular}

Forty-eight of 56 (data unknown on 5 cases) patients received adjuvant systemic therapy and 17 of 54 (data unknown on 7 cases) received radiation. The average follow-up time was 37 months (range: $1-122$ months). Nine (15\%) patients recurred ( 7 distal and 2 loco-regional). Average time to recurrence was 43 months. The overall survival in the cohort was $74 \%(45 / 61)$. Of the 16 patients who died, only 5 died of breast cancer (all of these patients had distal recurrence) and 4 died of non-breast cancer causes (metastatic esophageal cancer, septic shock, metastatic colon cancer, and metastatic prostate cancer). In the seven patients in whom the cause of death was unknown, three had second primary cancers (glioblastoma, melanoma, lung cancer).

Conclusions: Male breast cancers are diagnosed at higher stage and appear to be biologically aggressive on morphology compared to female breast cancers. Despite these 
features, breast cancer specific survival appears to be similar to female breast cancer. However, overall survival seems to be impacted by second malignancies in patient with male breast cancer. The varied type of second malignancies suggests the possibility of other germ line mutations in addition to $B R C A 2$ in male breast cancer patients.

\section{Immunohistochemical Profile of Male Breast Carcinoma}

Kate M Serdy, David Dabbs, Rohit Bhargava. Magee-Womens Hospital of University of Pittsburgh Medical Center, Pittsburgh, PA.

Background: The immunohistochemical profile of female breast carcinoma has been previously characterized. Here we present the first comprehensive assessment of the immunohistochemical profile of male breast carcinoma.

Design: A panel of sixteen immunohistochemical stains was performed on tissue microarrays (TMAs) of 60 primary breast carcinomas with 3-fold redundancy. Tumor size was available from reports. Representative tumor slides from each case were reviewed prior to TMA construction and analyzed for Nottingham grade and tumor differentiation. Hormone receptor and HER2 status, previously performed, allowed further classification of tumors into four categories (53 ER-positive/HER2-negative, 5 ER-positive/HER2-positive, 1 ER-negative/HER2-positive, 1 ER-negative/HER2negative), reflecting different molecular categories. Markers were scored using the semiquantitative $\mathrm{H}$-score method.

Results: The individual marker positivity in decreasing order was $98 \%(54 / 55)$ for GATA-3, 97\% (58/60) for ER, 95\% (52/55) for AR, 90\% (54/60) for PR, 88\% (46/52) for DPC4, 75\% (41/55) for GCDFP-15, 72\% (39/54) for CK7, 55\% (30/55) for MGB, $15 \%(8 / 54)$ for VIM, $15 \%(8 / 55)$ for WT-1 and $10 \%(6 / 60)$ for HER2 . Seven markers were negative for all cases including CDX 2 (0/53), CK20 (0/54), Napsin (0/55), PAX8 $(0 / 55)$, PSA (0/54), TTF-1 (0/55), and UPII $(0 / 54)$. Data for all markers was available on 50 carcinomas (44 ER-positive/HER2-negative, 4 ER-positive/HER2-positive, 1 ER-negative/HER2-positive, and 1 ER-negative/HER2-negative), resulting in a total of 28 profiles. The most common profile was AR+/CDX2-/CK7+/CK20-/DPC4+/ER+/ GATA3+/GCD+/HER2-/MGB+/NAP-/PAX8-/PR+/PSA-/TTF1-/UPII-/VIM-/WT1-. In comparison to a dataset of female breast cancers (Gloyeske, N. et al. 2015;23:3), the immunoreactivity of GCDFP-15 in male breast cancers was significantly higher $(75 \%$ vs. $26 \%, \mathrm{P}=0.0001$ ). The reactivity of $\mathrm{CK} 7$ in male breast cancers was significantly lower than in female breast cancers $(72 \%$ vs $92 \%, \mathrm{P}=0.0002)$.

Conclusions: This immunohistochemical profile of male breast carcinoma will be an important resource to distinguish breast carcinoma from others in the setting of a tumor of unknown origin. Of note, this data highlights an unexpected pattern of immunoreactivity in one of the more commonly used antibodies in this setting, CK7, which was negative in $28 \%$ of male breast cancers. Twenty-eight unique profiles were identified in the current dataset, indicating that there is not a single specific marker for male breast carcinoma and that use of an immunohistochemical panel is warranted.

\section{Molecular and Histopathologic Correlation of BRCA1- and BRCA2-} Associated Invasive Breast Carcinoma

Tiffany Shao, Jordan Lerner-Ellis, Martin C Chang. Mt Sinai Hospital, Toronto, ON, Canada; University of Toronto, Toronto, ON, Canada.

Background: Mutations of $B R C A 1$ and $B R C A 2$ genes account for $\sim 20 \%$ of hereditary breast cancer (BC). BRCA1 and BRCA2 BCs tend to be high grade and HER2-negative. $B R C A 1 \mathrm{BCs}$ are more likely to be $\mathrm{ER} / \mathrm{PgR}$ negative than $B R C A 2 \mathrm{BCs}$, most of which are ER-positive. Many large studies have focused on histotype, grade, receptor status, and simple presence/absence of $B R C A$ mutations. Our aim was to correlate more specific data on both molecular variants and histomorphology.

Design: We identified patients with a pathogenic germline $B R C A$ variant and with $\mathrm{H} \& \mathrm{E} /$ IHC slides from a BC resection available. In total, 31 tumors were retrieved (age 47.4 y). We reviewed the slides, recorded clinicopathologic data and histologic features in detail. We correlated features with specific $B R C A$ variants. Luminal (Lum) was defined as ER-high (Allred 6-8), HER2-; basal-like (Bas) as ER-low (Allred score $\leq 5$ ), HER2-. Results: We found $18 B R C A 1$ and $13 B R C A 2$ associated $\mathrm{BCs}$. BC in $B R C A 1+$ did not differ from $B R C A 2+$ in tumor size, grade, focality, or nodal status. 11/18 BCs in $B R C A 1+$ had low ER; 8/13 BCs in BRCA2+ had high ER. BCs in BRCA1+ were less likely than $B R C A 2+$ to be PgR-high (0/18 vs 4/13) and HER $2+(1 / 18$ vs $3 / 13)$.

BRCA1 variants: $5 / 18$ were in Exon 11 , with a mixture of $\operatorname{Lum}(3 / 5)$ and Bas $(2 / 5)$. Lum $\mathrm{BC}$ was seen with whole-gene (1/1), Exon 8 (1/2), Exon $14(2 / 2)$. Exons $5(4 / 4), 8(1 / 2)$, and $20(3 / 3)$ were associated with Bas. Exon 16: 1/1 was HER2+.

BRCA2 variants: $9 / 13$ were in Exon 11, including Lum (4/9), Bas (2/9), and HER2+ (3/9). Exon 25: $2 / 2$ were Lum. Exons $15(1 / 1)$ \& 22 (1/1) were Bas. Variant class did not predict phenotype for $B R C A 1$ or $B R C A 2$.

$B R C A 2+\mathrm{BC}$ had more heterogeneity in tumor growth pattern and border. 1/13 BRCA2+ $(0 / 18 B R C A 1+)$ BCs was lobular. Bas in BRCA1+ $(\mathrm{N}=10)$ and $B R C A 2+(\mathrm{N}=4)$ did not differ in LVI, stromal/peritumoral, cytoplasmic/nuclear features, or mitoses; DCIS was present in more BRCA1+ Bas $(4 / 10$ vs $0 / 4)$. Lum in BRCA1+ $(\mathrm{N}=7)$ and $B R C A 2+(\mathrm{N}=6)$ did not differ in LVI, cytoplasmic or nuclear features, or DCIS; Lum in $B R C A 1+$ was associated with fibrosis $(2 / 7$ vs. $0 / 6)$, desmoplasia $(5 / 7$ vs. $2 / 6)$ and high mitoses (4/7 vs $1 / 6)$.

Conclusions: $\mathrm{BCs}$ associated with $B R C A 1$ and $B R C A 2$ are a heterogeneous group with clinicopathologic differences from sporadic $B C$ and each other. Variants in Exon 11 of both $B R C A$ genes (the largest protein-encoding regions) are associated with a wide range of phenotypes. Variants in other regions may result in more specific phenotypes. Further work is warranted to correlate tumor phenotypes to specific functional losses in the $B R C A$ genes.
281 Basal Biomarkers Nestin and INPP4b Accurately Identify Intrinsic Subtype in ER Weakly-Positive Breast Cancer

Brandon S Sheffield, Karama Asleh-Aburaya, Xiuqing Wang, Dongxia Gao, Jennifer Won, Christine Chow, Robert Wolber, Blake Gilks, Nickolas Myles, Malcolm M Hayes, Philip Bernard, Torsten O Nielsen, Stephen KL Chia, Zuzana Kos. University of British Columbia, Vancouver, BC, Canada; University of Utah, Salt Lake City, UT; University of Ottawa, Ottawa, ON, Canada.

Background: Breast cancer intrinsic subtypes, determined by gene-expression profiling, offer valuable prognostic and predictive information. Gene expression studies are expensive and not readily available, making immunohistochemical (IHC) surrogates an attractive alternative. Recent evidence indicates that ER weak positivity by IHC is not reliably associated with luminal subtype. Expression of nestin and loss of INPP4b (inositol polyphosphate-4-phosphatase) have been shown to be the IHC markers most strongly associated with the basal-like subtype. We assessed their capacity to discriminate intrinsic subtypes by IHC, focusing on clinically problematic cases with weakly positive ER expression.

Design: A cohort of invasive breast carcinomas, enriched for ER-negative (Allred 0,2) and weakly positive (Allred 3-5) cases, was identified through retrospective chart review. Tumor-rich areas were identified for tissue-microarray (TMA) construction, nucleic acid extraction for qRT-PCR, and intrinsic subtype prediction using the PAM50 classifier. TMA slides were assessed for CK5, EGFR, nestin, and INPP4b by IHC. Clinical ER, PR and HER2 status were extracted from the patient charts.

Results: 206 cases were included, among which 101 (49\%) were basal-like by PAM50. Out of 58 cases weakly-positive for ER, only $9(16 \%)$ were assigned to luminal subtypes by PAM50, whereas $28(48 \%)$ were classified as basal-like. Nestin and INPP4b showed improved test characteristics to the previously described basal-like IHC markers CK5 and EGFR. In the setting of weakly positive ER staining and HER 2 negative status, these markers were able to predict basal-like subtype with a sensitivity of $71 \%$, specificity of $90 \%$, PPV of $95 \%$ (C.I. $88 \%-100 \%$ ) and can exclude luminal subtype with an NPV of $95 \%$ (C.I. $88 \%-100 \%$ ).

Conclusions: A large fraction of ER weakly positive tumors are of basal-like rather than luminal intrinsic subtype. Nestin and INPP4b offer superior sensitivity over previously proposed basal-like IHC markers. These markers allow for more accurate IHC-based subtyping, and are particularly useful in the setting of ER weak positivity in identifying patients who have a molecular subtype that stands to benefit more from adjuvant chemotherapy than from hormonal therapy.

\section{The Prognostic Value of E-Cadherin and $\beta$-Catenin in Triple- Negative Breast Cancer (TNBC)}

Tiansheng Shen, Gene P Siegal, Shi Wei. University of Alabama at Birmingham, Birmingham, AL.

Background: TNBC is generally associated with a poor clinical outcome, for which the only systemic treatment option is cytotoxic chemotherapy. Subclassifying TNBC with differing prognostic significance thus should be of benefit in patient management. E-cadherin (E-cad) and $\beta$-catenin $(\beta$-cat) are cell adhesion molecules and members of the cadherin complex that play a crucial role in the progression of various tumor types, including BC. The membrane-uncomplexed $\beta$-cat and its subsequent nuclear/ cytoplasmic over-accumulation is thought to play a key role in BC tumor biology as a result of altered Wnt signaling. The aim of this study was to assess the prognostic significance of E-cad and $\beta$-cat in TNBC.

Design: TNBCs in the authors' institution with pre-chemotherapy tissue available were retrieved. Pleomorphic lobular BC and those with metastasis at diagnosis were excluded. The clinicopathologic parameters were recorded, including age, race, tumor size and nodal status, along with survival outcomes. The intensity of membranous E-cad and $\beta$-cat ( $\mathrm{mE}$-cad/m $\beta$-cat) was scored as 3 (strong; equivalent to adjacent normal epithelium), 2 (moderate), 1 (weak) or 0 (no staining). A score of $\leq 2$ was regarded as reduced expression. Cytoplasmic/nuclear subcellular localization of $\beta$-cat was also examined. Results: Of 73 TNBCs meeting the inclusion criteria, 41 (56\%) and 41 cases showed reduced $\mathrm{mE}$-cad or $\mathrm{m} \beta$-cat, respectively, with a strong correlation with each other $(\mathrm{P}<0.0001)$. Of those with reduced $\mathrm{m} \beta$-cat, cytoplasmic localization of the protein was found in $4(10 \%)$ cases, whereas no nuclear expression was observed in any case. Of clinicopathologic factors analyzed, tumor size and nodal status were significantly associated with overall survival (OS) and disease specific survival (DSS), respectively $(\mathrm{P}<0.0001$ for both). Either reduced $\mathrm{mE}$-cad or $\mathrm{m} \beta$-cat were significantly associated with a poor OS ( $\mathrm{P}=0.02$ and 0.049 , respectively) and $\mathrm{DSS}(\mathrm{P}=0.01$ and 0.01 , respectively). The expression of $\mathrm{mE}$-cad and $\mathrm{m} \beta$-cat were not significantly correlated with the other pathologic factors analyzed.

Conclusions: E-cad and $\beta$-cat expression provides discriminative prognostic power independent of conventional pathologic factors, thus further reinforcing the important role of cell adhesion molecules in the process of tumor metastasis, especially in TNBC. The fact that no nuclear translocation was found in any case and only a minimal proportion of cases showed cytoplasmic localization in the subset of tumors with reduced $\mathrm{m} \beta$-cat suggests that molecular pathways other than $\beta$-cat/Wnt signaling are essential in the progression of TNBC.

\section{Characterization of Estrogen Receptor Positive/Progesterone} Receptor Negative/(ER+/PR-) Breast Cancer (BC)

Tiansheng Shen, Tao Guo, Omar Hameed, Gene P Siegal, Shi Wei. University of Alabama at Birmingham, Birmingham, AL.

Background: Although approximately $70 \%$ of $\mathrm{BCs}$ are $\mathrm{ER}+$, the $\mathrm{ER}+/ \mathrm{PR}$ - phenotype had a reported incidence of $12-20 \%$ despite the fact that PR is an ER-dependent gene product. We and others have previously demonstrated that the ER+/PR- BCs were associated with a worse clinical outcome when compared to the $\mathrm{ER}+/ \mathrm{PR}+$ tumors 
in the entire cohort or in those receiving endocrine therapy (ET). However, detailed information on the clinicopathologic factors of ER+/PR- BCs is largely lacking. The aim of this study was to characterize the pathologic features and prognostic significance of $\mathrm{ER}+/ \mathrm{PR}-\mathrm{BCs}$ in comparison to $\mathrm{ER}+/ \mathrm{PR}+$ tumors.

Design: The tumor registry of the authors' institution was searched to identify BCs from 1998 to 2013. The clinicopathologic parameters of the primary BCs were recorded, along with therapeutic modalities and outcomes. Those with metastasis at diagnosis / Stage IV were excluded. Analysis of distant relapse free survival (RFS) was performed by using the Kaplan-Meier method and the log-rank test.

Results: Of 5328 invasive BCs with known ER/PR status, $3104 \mathrm{ER}+/ \mathrm{PR}+$ and $636 \mathrm{ER}+/$ PR- BCs met the inclusion criteria. The proportion of BCs with a HER2+ phenotype was significantly higher in the ER+/PR- tumors $(24.5 \%$ vs $14.4 \%, \mathrm{P}<0.0001)$. Factors significantly associated with RFS in ER+/PR- BCs included age, tumor grade and size, HER2 and nodal status either in the entire cohort or in the subset of patients who received ET. When compared to the ER+/PR-/HER2- BCs, the ER+/PR+/HER2- tumors were associated with a significantly prolonged RFS in all age groups, in Caucasians, in Grade I/II tumors, and in all stages; whereas a significant survival benefit of the ER+/ $\mathrm{PR}+/ \mathrm{HER} 2+$ phenotype was only seen in postmenopausal women when compared to ER+/PR-/HER2+ BCs. However, in the subset of patients receiving ET, a significantly favorable RFS associated with ER+/PR+/HER2- BCs was seen in premenopausal women and in locally advanced BCs (Stage IIB/III) when compared to ER+/PR-/ HER2- tumors, while the ER+/PR+/HER2+ phenotype was associated with a significant survival advantage in postmenopausal patients, in all races, in Grade III tumors, and in early stage (I/IIA) BCs when compared to ER+/PR-/HER2+ tumors.

Conclusions: Although the current treatment for ER+ tumors is similar, the ER+/PR$\mathrm{BCs}$ are clinically and probably genetically different from the $\mathrm{ER}+/ \mathrm{PR}+$ tumors. The ER+/PR- phenotype suggests aberrant growth factor signaling that could contribute to ET resistance. Individualized chemotherapy should strongly be considered in early stages based on additional compounding factors as elucidated above.

\section{Clinicopathologic Factors Associated with Metastatic Breast} Cancer (BC) at Presentation

Tiansheng Shen, Gene P Siegal, Shi Wei. University of Alabama at Birmingham, Birmingham, AL.

Background: Approximately $20-30 \%$ of patients will develop a distant recurrence despite advancements in the treatment of early BC. Metastatic BC is a largely incurable disease, with a median survival of less tnan 3 years. Current practice guidelines suggest utilizing the stage at presentation to guide initial imaging decisions regarding detecting such distant metastasis, although controversies remain. In this study, we sought to determine if specific clinicopathologic factors were significantly associated with distant metastasis at presentation.

Design: The tumor registry of the authors' institution was searched to identify BCs from 1998 to 2013. The clinicopathologic parameters of the primary BCs were recorded, including patient's age and race, tumor type, histologic grade, ER/PR/HER2 status, and clinical stage at presentation. Chi-Square test and logistic regression models were utilized to examined if selected factors were associated with metastasis at diagnosis. Results: Of the 6128 BCs included in the study period, 618 developed distant metastases either at the time of presentation $(n=253)$ or subsequently $(n=365)$. The clinicopathologic factors significantly associated with metastasis at diagnosis included race, high histologic grade, ER-, PR-, and HER2+ status in the cohort. When analyzing the subset of patients with locally advanced BC (Stage IIB/III) at presentation, postmenopausal women, high histologic grade and PR- and HER2+ status were significantly associated with metastasis at diagnosis. The subtype with the highest proportion of metastasis at diagnosis was ER-/ PR-/HER2 + in the entire cohort $(18.4 \% ; \mathrm{P}<0.0001)$ or in locally advanced BC $(43 \%$; $\mathrm{P}<0.0001)$. Metastasis at presentation had a significantly worse overall survival when compared to those with metastatic recurrence in this cohort $(\mathrm{P}<0.0001)$, in contrast to a recent study demonstrating that de novo metastatic BCs had a significantly better outcome than those with recurrent metastasis [Br J Cancer 2015;112:1445].

Conclusions: The current ASCO surveillance guidelines for early $\mathrm{BC}$ recommends regular follow-up with a history, physical examination and mammography, but no routine laboratory or other imaging studies. Our data suggest that an algorithm incorporating pathologic factors, especially histologic grade and receptor profile, may be of benefit for decision making to perform a clinical work-up in newly diagnosed BC in the pursuit of precision medicine. Moreover, PR status demonstrated a more powerful predictive value than ER in this setting, thus further underlying the importance of routine testing for this biomarker.

\section{Expression of the Autophagy Protein p62 in FEA Does Not Predict for Upgrading to Atypical Hyperplasia or Carcinoma}

Jordan Sim, Jason K Wasserman, Susan J Robertson. The Ottawa Hospital, Ottawa, ON, Canada.

Background: Flat epithelial atypia (FEA) is an atypical lesion of the breast which is viewed as a non-obligate precursor of low grade carcinomas. In $4.1 \%$ of biopsies, FEA is the highest grade lesion (so-called "pure FEA"). Many studies have been done to assess whether excision is warranted for a diagnosis of pure FEA. Upgrade rates to a diagnosis of DCIS or higher have ranged from $6.9-18.9 \%$. Attempts to predict which patients with FEA will progress or result in upgrading on excision have unfortunately met with limited success.

P62 is an autophagy-related protein involved in many cellular pathways. In breast cancer, p62 is significantly upregulated in comparison to normal breast epithelial cells. It has also been correlated with breast cancer grade, rate of distant metastasis, EGFR expression and HER2 expression. Further p62 is an important NF-kappaB mediator in tumorigenesis.
Given our previous identification that IKKepsilon, an NF-kappaB pathway signalling protein, was a predictor for upgrading in FEA; we hypothesized that p62 staining would also correlate with the rate of upgraded diagnosis in FEA.

Design: Pathology reports for partial and complete mastectomy specimens from 20112014 were searched for a reported diagnosis of FEA. 70 patients were discovered. The mastectomy specimens were reviewed, and all blocks with FEA were pulled and stained with p62 (a total of 88 blocks). 1 senior pathologist and 1 junior resident, blinded to any concurrent diagnoses, then reviewed all p62-stains and gave each a score from 1 to 3 for intensity and 1 to 6 for percentage of the total nuclei that stained $(1=<1 \%, 2$ $=1-10 \%, 3=10-25 \%, 4=25-50 \%, 5=50-75 \%, 6=>75 \%)$. These scores were then added together and each block was given a total score out of 9 . The pathology reports from the specimens were then reviewed to assess for the presence of concurrent higher grade lesions. $39(55.7 \%)$ patients had an upgrade to at least DCIS and $31(44.3 \%)$ had FEA, ALH or ADH as the highest grade lesion. A 2-tailed t test was performed to assess whether there was any difference in p62 scores between these two groups. Results: The results of the t-test show no significant difference $(\mathrm{p}=0.438)$ in $\mathrm{p} 62$ scores between those cases with a concurrent upgraded diagnosis versus those without (mean 6.05 vs. 5.48).

Conclusions: This is the first time that $\mathrm{p} 62$ has been investigated as a predictor of upgraded diagnosis in FEA. Our study concludes that p62 is not a useful predictor.

286 Incidence of Axillary Metastasis in Breast Carcinomas Measuring $1 \mathrm{~cm}$ or Less

Reed Spaulding, Patrick C McGrath, Joanna Dalland, Robert McDonald, Shannon Crook, Luis Samayoa. University of Kentucky, Lexington, KY.

Background: Earlier studies have shown that depending on their size and grade of differentiation, some breast carcinomas rarely metastasize to the axilla. When they do, they show minimal lymph node (LN) involvement (1-3 LN). Our study identifies a subset of patients that in the context of the ACOSOG-Z11 trial could potentially be managed with lumpectomy and radiation without sentinel node (SN) biopsy.

Design: We identified 369 patients with $\leq 1.0 \mathrm{~cm}$ breast cancers who underwent $\mathrm{SN}$ biopsy from $2000-2015$. Patients were classified according to the following: type of surgery (lumpectomy vs. mastectomy), tumor size (Tmic, T1a, or T1b), tumor grade (modified Bloom-Richardson), number and status of SN and non-sentinel lymph nodes (NSN), size of SN tumor deposits ( $\leq 5 \mathrm{~mm}$ or $>5 \mathrm{~mm})$, Her $2 /$ neu status, and hormone receptor (HR) status. Tumor size, grade, hormonal and her2-neu status were then correlated with final axillary stage. Statistical analysis was performed on the results. Results: Table 1. Axillary Stage in Breast Cancer Patients with $\leq 1 \mathrm{~cm}$ Tumors

\begin{tabular}{|l|l|l|l|l|l|}
\hline & N0 & Total (+) Axillas & N0(itc) & N1mi & N1a \\
\hline Mastectomy (35.9\%) & 108 & 24 & 3 & 12 & 9 \\
\hline Lumpectomy (64.1\%) & 218 & 18 & 2 & 7 & 9 \\
\hline \multicolumn{7}{|l|}{ T1mi * (2.2\%) } & 8 & 0 & 0 & 0 & 0 \\
\hline T1a (19.0\%) & 65 & 5 & 0 & 3 & 2 \\
\hline T1b (78.8\%) & 253 & 37 & 5 & 16 & 16 \\
\hline \multicolumn{7}{|l|}{} & 13 & 6 & 8 \\
\hline Grade I (39.4\%) & 130 & 15 & 1 & 9 & 7 \\
\hline Grade II (43.7\%) & 144 & 17 & 1 & 4 & 3 \\
\hline Grade III (14.7\%) & 44 & 10 & 3 & \\
\hline *T1mi cases were not given a modified Bloom Richardson score. \\
\hline
\end{tabular}

Table 2. Axillary Stage in Breast Cancer Patients with $\leq 1 \mathrm{~cm}$ Tumors According to Hormonal and Her2 Status

\begin{tabular}{|l|l|l|l|l|}
\hline & N0 & N0(itc) & N1mi & N1a \\
\hline ER+/HER2- (75.1\%) & 214 & 2 & 16 & 12 \\
\hline ER+/HER2+ (6.5\%) & 17 & 0 & 1 & 3 \\
\hline ER-/HER2+ (5.5\%) & 12 & 3 & 0 & 3 \\
\hline ER-/HER2-(12.9\%) & 40 & 0 & 2 & 0 \\
\hline
\end{tabular}

Only 18 (4.9\%) patients had macrometastatic disease, all limited to 1 and/or 2 LN. The majority of tumor deposits were $\leq 5 \mathrm{~mm}(97.5 \%)$. There was no statistical significance between the tumor grade and the incidence of metastasis; however, ER-/Her2+ tumors were more likely to have LN metastases than ER+/Her2- tumors $(p=0.012)$.

Conclusions: Regardless of HR and Her2 status or tumor grade, breast cancer patients with $\leq 1 \mathrm{~cm}$ tumors rarely have axillary metastases. In the context of the ACOSOG-Z11 results, their management could be based primarily on their biologic markers, potentially with lumpectomy and radiation alone. 
287 Analysis of Clinical-Pathological Data with Impact on Overall Survival (OS) in Male Breast Carcinoma: An International Multi-Institutional Study of 220 Cases

Simona Stolnicu, Denisa Moncea, Alis Dema, Rares Buiga, Cosmin Moldovan, Rares Georgescu, Cornelia Toganel, Septimiu Voidazan, Erika Resetkova, Maria Comanescu, Isabel Alvarado-Cabrero. University of Medicine and Pharmacy Tg Mures, Targu Mures, Romania; University of Medicine "Victor Babes", Timisoara, Romania; Oncologic Institute "I. Chiricuta", Cluj Napoca, Romania; MD Anderson Cancer Center, Houston, TX; "Victor Babes" Institute, Bucuresti, Romania; Oncologic Institute, Mexico City, Mexico.

Background: Male breast carcinoma is still inadequately characterized and data regarding the impact of different prognostic parameters are limited due to the small number of cases compared to female counterpart. The objective of this study is to evaluate clinical-pathological parameters in correlation with OS in male breast carcinoma.

Design: All men diagnosed with invasive breast carcinoma in 6 institutions (1999-2015) were identified by searching laboratory databases. Parameters analyzed: age, clinical and pathological stage, histological tumor type and grade, molecular biomarkers (ER, PR, Ki-67, HER2), treatment and length of OS.

Results: 220 cases, a mean age of 62 (range: $30-85$ ), NST histological type (83\%), G2 histological grade (54\%), T2 (76\%), N2 (27\%), M0 (25\%) and Luminal A molecular subtype (56\%). ER and PR were positive in $91 \%$ and $86 \%$ of cases, respectively. HER-2 was overexpressed in $10 \%$ of cases and a high $\mathrm{Ki}-67$ proliferation index was present in $85 \%$ of cases. Follow-up (FU) was available for 155 patients, with a median FU of 144 months (range: 10-180 months). 15 -year OS was $80 \% ; 104$ pts are alive, 51 died of disease and 3 alive with metastasis. In univariate analysis higher histological grade, NST subtype and triple negative cases were associated with worse OS $(\mathrm{p}=0.05,0.002$ and 0.01 , respectively) while in the multivariate analyses OS is independently influenced only by age over 60 years (p: $0.04,95 \%$, CI: $1.98-8.88$, OR: 2.96 ). No statistical difference was identified in OS between institutions or continents.

Conclusions: Age over 60 is the only statistically significant unfavorable independent prognostic parameter in multivariate analysis in male breast carcinomas.

288 Androgen Receptor Status and Prediction of Neoadjuvant Chemotherapy Response in Triple-Negative Breast Cancer

Catherine Stoos, Shannon Huggins-Puhalla, David Dabbs, Rohit Bhargava. MageeWomens Hospital, Pittsburgh, PA.

Background: While approximately one-third of triple-negative primary breast cancers (TNBC) achieve pathologic complete response (pCR) to neoadjuvant chemotherapy, there are no reliable morphologic or immunohistochemical (IHC) markers to predict this response. Androgen receptor (AR) status has been previously shown to correlate with prognosis in TNBC. Here, we evaluate AR immunoreactivity by IHC as a potential marker of tumor volume reduction (TVR) and pCR in TNBC.

Design: We evaluated IHC AR expression on a tissue microarray created from 56 preneoadjuvant chemotherapy TNBC core biopsy specimens. Due to tissue loss during processing, only 42 cases were used in the final AR scoring. AR immunoreactivity semiquantitation was performed using the H-score method, which is the sum of the staining percentage multiplied by a corresponding staining intensity value $(0=$ none, $1=$ weak, $2=$ moderate, $3=$ strong). Then, AR positivity was correlated with the occurrence of TVR $\geq 50 \%$ and $\mathrm{pCR}$ in the resection specimen using 2 different cut-off values for AR positivity $(\mathrm{H}$-score $>1$ and $\mathrm{H}$-score $>10$ ). The average percent TVR (using the Magee Method) based on AR result was also evaluated. Significance was determined using Fisher exact test and unpaired T-tests, with $P$ values of $\leq 0.05$ considered significant. Results: There was no difference in $\mathrm{pCR}$ rates between AR positive and AR negative tumors, regardless of the cut-off used to define the AR positivity.

\begin{tabular}{|l|l|l|l|}
\hline & No pCR & pCR & Total \\
\hline $\mathrm{AR}(-)$ & 22 & 11 & 33 \\
\hline $\mathrm{AR}(+)$ & 7 & 2 & 9 \\
\hline Grand Total & 29 & 13 & 42 \\
\hline $\begin{array}{l}\text { AR cut-off used was H-score }>10 \\
\text { pCR in AR(-): 11/33 [33\%]; pCR in AR(+): 2/9 [22\%]; } P=0.6953\end{array}$ \\
\hline
\end{tabular}

When the data was analyzed for TVR, a trend for AR negative cases with $\geq 50 \%$ TVR was noted. This difference was seen only using an AR positive cut-off $\mathrm{H}$-score of $>10$.

\begin{tabular}{|l|l|l|l|}
\hline & $\mathbf{5 0 \%}$ TVR & $\mathbf{2 5 0 \%}$ TVR & Total \\
\hline AR(-) & 9 & 23 & 32 \\
\hline AR( $(+)$ & 6 & 3 & 9 \\
\hline Grand Total & 15 & 26 & $41^{*}$ \\
\hline * TVR not available for 1 case. AR cut-off used was H-score $>10$. \\
$\begin{array}{l}\geq 50 \% \text { TVR in AR(-): } 23 / 32[72 \%] ; \geq 50 \% \text { TVR in AR }(+): 3 / 9[33 \%] ; P=0.0525 . \\
\text { The average percent TVR in AR negative tumors was } 71 \% \text { compared to } 47 \% \text { in AR positive } \\
\text { tumors }(P=0.0874) .\end{array}$ \\
\hline
\end{tabular}

Conclusions: While not statistically significant, the data show a trend towards AR negative tumors showing a TVR of $\geq 50 \%$ when AR positivity by IHC is defined as an $\mathrm{H}$-score of $>10$. Therefore, an AR $\mathrm{H}$-score positivity of $>10$ by IHC may serve as a potential marker for assessing response to neoadjuvant chemotherapy in TNBC. A larger volume of cases is needed for further evaluation.
289 High Subcapsular CD4/CD8 Lymphocyte Ratio in the Positive Sentinel/Axillary Lymph Node in Breast Cancer

Hongxia Sun, Min Li, Yan Gao, Robert L Hunter, Songlin Zhang. The University of Texas Health Science Center at Houston, Houston, TX.

Background: Studies have reported that tumor-infiltrating lymphocytes (TILs) can affect the prognosis and therapeutic response of breast cancer. CD8(+) cytotoxic T cells and $\mathrm{CD} 4(+)$ regulatory $\mathrm{T}$ cells are two of the main players of immune surveillance and tolerance respectively. So far, there are very few studies about the CD4 and CD8 lymphocytes in sentinel/axillary lymph nodes of breast cancer patients. The goal of this study is to correlate the CD4, CD8 count and ratio with status of sentinel/axillary lymph nodes.

Design: Thirty-two sentinel/axillary lymph nodes from breast cancer patients including fourteen positive and eighteen negative for metastasis were included in the study. Immunohistochemistry was performed by using $\mathrm{CD} 4+\mathrm{CD} 8$ double staining primary antibody (Clone BC/1F6, BioCare), MACH2 double staining secondary antibody, and DAB and Vulcan Fast Red Chromogens. For each case, three images were taken from each subcapsular, cortical and follicular region by using Nuance multispectral imaging system (Version 3.0.1, Caliper life sciences). The total lymphocytes, CD4 or CD8 positive lymphocytes in each image were counted using inForm advanced image analysis software (Version 2.1.1, Perkin Elmer). The percentage of CD4 or CD8 positive lymphocytes and CD4/CD8 ratio in each region were calculated. Student $t$ test was used for statistic analysis

Results: Comparing to the lymph nodes without metastatic carcinoma, the lymph nodes with metastatic cancer have significantly increased CD4 lymphocytes $(67.8 \pm 15.4 \%$ vs. $51.5 \pm 10.7 \%, \mathrm{p}=0.0014)$, decreased CD8 lymphocytes $(7.15 \pm 5.52 \%$ vs. $10.7 \pm 4.04 \%$, $\mathrm{p}=0.042)$, and increased $\mathrm{CD} 4 / \mathrm{CD} 8$ ratio $(16.7 \pm 11.6 \%$ vs. $5.81 \pm 3.74, \mathrm{p}<0.001)$. There are no significant differences on $\mathrm{CD} 4, \mathrm{CD} 8$, or $\mathrm{CD} 4 / \mathrm{CD} 8$ ratio in the cortical and follicular regions between the two study groups.

Conclusions: Our study shows that sentinel/axillary lymph nodes with metastatic cancer have decreased CD8 cytotoxic and increased CD4 regulatory lymphocytes in the subcapsular region. Subcapsular region is the space where metastatic tumor cells first encounter with the lymphocytes in a lymph node. It is not clear whether this change is the result of tumor metastasis or is one of the factors facilitating tumor metastasis. This finding supports the role of host immune response in breast cancer metastasis. We are currently investigating its association with TILs in tumor tissue.

290 Suggested Management of Breast Sclerosing Intraductal Papilloma without Atypia (SIP w/o A) Diagnosed on Core Biopsy (CBX): A Semiquantitative Approach

Brittany Symbol, Andrew Ricci. Hartford Hospital, Hartford, CT.

Background: While there is general agreement for surgical excision following CBX of SIP with atypia, a consensus for SIP w/o A is lacking. Studies have addressed this issue in an effort to identify surgical candidate lesions, with varying results. The upgrade risk to atypical duct hyperplasia \& ductal carcinoma in situ on excision has driven aggressive surgical management. The aim of this study was two-fold: 1) To examine a consecutive series of CBX (SIP w/o A) followed by surgical excision with special attention to estimating the extent of lesional sampling by direct Radiologic-Pathologic (Rad-Path) correlation; \& 2) To provide baseline comparative data of excised SIP examining the risk of atypia stratified by lesional size.

Design: For the first aim consecutive paired specimens (CBX + excision) were identified from a two year period. All slides were reviewed. The area of papilloma was calculated $\left(\mathrm{A}=\Pi^{2}\right)$ \& Rad-Path correlation with size of the ultrasound targeted lesion was performed. For the second aim we gathered 5-year data (reports from COPATH $\AA$ database) from our institution for all excised SIP noting the \% of cases with atypia. We then examined the subset of cases reporting lesional size \& stratified these (with $\&$ without atypia) by linear dimension. All slides from this subset were reviewed. Results: For the first aim consecutive paired $\mathrm{CBX}$ and excisional specimens ( $\mathrm{N}=13$ ) were collected from 2011-2012. All were SIP w/o A on CBX and none showed atypia on excision. There was good Rad-Path correlation with regard to lesional size. For

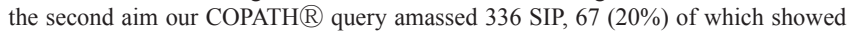
atypia. For 187 cases a lesional size was recorded. This became the subset of cases for the development of our baseline comparative data. When SIP were stratified by size $8 / 76(12 \%) 1-5 \mathrm{~mm}$ showed atypia, 20/134 (15\%) 1-10mm showed atypia, \& 25/154 (16\%) 1-12mm showed atypia; $12 / 33$ (36\%) of SIP sized $13-40 \mathrm{~mm}$ showed atypia. Conclusions: Combining the two phases of this report we conclude: 1) Calculation of lesional area shows good correlation with radiologic size; 2) With regard to our paired CBX-excision study based on our comparative data we would have expected $12-16 \%$ of papillomas of similar size to show atypia; 3 ) If a CBX "substantially" samples a SIP, shows no atypia, and has good Rad-Path size correlation, it is unlikely to be upgraded on excision; 4) Reflex excision of SIP w/o A diagnosed on CBX (meeting these criteria) is not recommended (although surgical excision may still be appropriate in selected cases).

291 High EZH2 Expression Accompanied by Low H3K27me3 Predicts Poorer Outcome in Metaplastic Carcinoma of the Breast

Benjamin Yongcheng Tan, Clara Ong, Valerie Koh, Aye Aye Thike, Puay-Hoon Tan. Singapore General Hospital, Singapore, Singapore.

Background: Metaplastic breast carcinoma (MBC) encompasses a heterogeneous group of tumors accounting for $0.2 \%$ to $5 \%$ of all breast cancers. Most metaplastic carcinomas have lower response rates to conventional adjuvant chemotherapy and a worse clinical outcome. EZH2 (Polycomb histone methyltransferase enhancer of zeste homolog 2) regulates a variety of cellular processes, including cell proliferation, apoptosis, invasion and self-renewal, and is a validated marker of aggressiveness and 
poor outcome in many tumors. EZH2 catalyzes histone H3 lysine 27 tri-methylation (H3K27me3) which is associated with transcriptional repression. We investigate the role of protein expression of EZH2 and $\mathrm{H} 3 \mathrm{~K} 27 \mathrm{me} 3$ in MBC.

Design: The study cohort comprised $85 \mathrm{MBCs}$ diagnosed at the Department of Pathology, Singapore General Hospital from 1994 to 2013. Pathologic parameters of tumor size, grade, lymph node stage, and metaplastic components were reviewed. Immunohistochemistry was performed on tissue microarray blocks using antibodies to ER, PR, HER2 (to detect triple negativity), CK14, 34ßE12, EGFR (to define basallike phenotype), EZH2 and H3K27me3. Positive biomarker positivity was defined as $\mathrm{H}$-scores of 50 or more of EZH2 and H3K27me nuclear staining. Clinicopathological parameters and survival outcomes were correlated with protein immunoexpression. Results: T2 stage was observed in $75 \%$ of tumors, with predominantly grade 3 cancers $(69 \%)$. Triple negative phenotype was seen in $86 \%$ and basal-like subtype in $72 \%$ of tumors. Expression of EZH2 was observed in 37 (43\%) and H3K27me3 in 18 (21\%) tumors. Increasing EZH2 expression was noted in high grade tumors $(\mathrm{p}=0.077)$. HER2 and CK14 positive tumors were significantly associated with positive EZH2 expression $(\mathrm{p}=0.019$ and $\mathrm{p}=0.007)$. Triple negative phenotype was significantly correlated with $\mathrm{EZH} 2+\mathrm{H} 3 \mathrm{~K} 27 \mathrm{me} 3-$ tumors. Mean and median follow up were 57 and 40 months, with a maximum of 219 months. Combinational phenotype (EZH2+H3K27me3-) was observed in $23(27 \%)$ tumors. Cases with this phenotype showed poorer overall survival $(p=0.021)$. Multivariate analysis confirmed statistically significant association of the combinational phenotype with unfavorable outcome (HR 7.244, 95\% CI 1.56033.626, $\mathrm{p}=0.011$ ).

Conclusions: $\mathrm{MBCs}$ are usually high grade $\mathrm{T} 2$ tumors, predominantly triple negative with basal-like phenotype. A combinational EZH2+H3K27me3-phenotype was associated with triple negativity. Patients whose tumors harbored this combinational phenotype showed poorer outcome. Our findings may provide new insights for targeted therapy especially in triple negative MBCs.

292 Role of Biomarkers (CD44, E-cadherin and $\beta$-catenin) in Predicting Recurrence and Progression of Ductal Carcinoma In Situ of the Breast Benjamin Yongcheng Tan, Clara Ong, Valerie Koh, Aye Aye Thike, Puay-Hoon Tan. Singapore General Hospital, Singapore, Singapore.

Background: Ductal carcinoma in situ (DCIS) of the breast accounts for 20 to $25 \%$ of newly diagnosed breast cancers. To date, there is no consensus on biomarkers that can predict recurrence and progression of DCIS. We aimed to evaluate immunohistochemical expression of CD44, E-cadherin and $\beta$-catenin in DCIS with and without subsequent recurrent disease.

Design: The study cohort comprised 198 pure DCIS cases, consisting of 101 tumors with known recurrent disease, and 97 non-recurrent DCIS as controls. Immunohistochemistry was performed on standard sections to which antibodies to CD44, E-cadherin and $\beta$-catenin were applied, using the streptavidin-biotin method. Positive biomarker expression was defined as staining of $10 \%$ or more of membranous decoration for CD44, H-scores of 100 or more for membranous E-cadherin and for cytoplasmic and membranous $\beta$-catenin expression. Disease free survival (DFS) was defined as time from diagnosis to recurrence or date of last follow up, and correlated with protein immunohistochemical expression.

Results: CD44 expression was observed in $10.9 \%$ of cases of DCIS with recurrence and $24.7 \%$ in those without $(\mathrm{p}=0.010)$. Loss of membranous E-cadherin expression was found more frequently in DCIS with recurrence than in the control group $72.3 \% \mathrm{vs}$ $51.5 \%)(\mathrm{p}=0.003)$. No significant difference was found for membranous and cytoplasmic $\beta$-catenin expression between the two groups ( $28.7 \% \mathrm{Vs} 40.2 \%$ and $29.7 \% \mathrm{Vs} 39.2 \%$ ). Patients who harbored DCIS with loss of E-cadherin expression had a greater likelihood of early recurrence (HR 1.595, 95\% CI 1.018-2.499, $\mathrm{p}=0.042$ ). Women with DCIS displaying a combination of negative CD44 staining and loss of membranous E-cadherin expression also disclosed poorer DFS (HR 13.483, 95\%CI 1.872-97.112, $\mathrm{p}=0.010$ ).

Conclusions: Our study demonstrates that loss of membranous E-cadherin expression, as well as negative CD44 immunostaining, predict DCIS recurrence. Our study suggests that CD44 has a tumor suppressor role in early breast cancer (DCIS). A combinational phenotype of CD44 negativity with loss of membranous E-cadherin expression is significantly associated with early DCIS recurrence and progression.

293 PD-L1 Expression and the Immune Microenvironment in Primary Invasive Lobular Carcinomas of the Breast

Elizabeth Thompson, Janis Taube, Rebecca J Asch-Kendrick, Aleksandra Ogurtsova, Haiying Xu, Rajni Sharma, Alan Meeker, Pedram Argani, Leisha Emens, Ashley CiminoMathews. Johns Hopkins Hospital, Baltimore, MD.

Background: The tumor immune microenvironment is increasingly recognized as a potential prognostic factor and therapeutic target in breast cancer. Most studies have focused on invasive ductal carcinomas, with limited attention to special tumor subtypes. Here, we investigate the tumor microenvironment in 47 cases of primary breast invasive lobular carcinomas (ILC).

Design: Tissue microarrays (TMAs) containing 47 ILC (1.4 mm cores, 5-10/per tumor) were labeled by immunohistochemistry for PD-L1, CD8, CD20 and FoxP3. The percentage ILC carcinoma cells with clear membranous PD-L1 expression was scored in $5 \%$ increments, with $<5 \%$ labeling considered negative. The percentage PD-L1 expression by TILs was scored as none $(0)$, focal $(1+;<=5 \%)$, moderate $(2+$; $10-24 \%)$, or diffuse $(3+; 50-100 \%)$. The TIL infiltrate density was scored as none (1), mild ( 1 ; rare TIL $<5 \%$ of tumor area), moderate ( 2 ; focal infiltrate, $5 \%$ of tumor area), and brisk ( $3 ;>=10 \%$ of tumor area).

Results: The 47 ILC consisted of 33 ER+/HER2-, 8 ER+/Her2+, 1 ER-/HER2+, and 5 ER-/HER2-carcinomas; 5 were Grade I, 34 grade II, and 8 grade 3 . All ILC contained TIL infiltrate, but the majority (53\%) were focal; $32 \%$ were moderate and $15 \%$ were brisk. All ILC contained PD-L1+ TIL, with $11 \%$ focal, $60 \%$ moderate and $30 \%$ diffuse.
17\% ILC had PD-L1+ carcinoma cells. Tumors with brisk TIL infiltrate had higher PD-L1+ TIL than those with focal TIL $(\mathrm{p}=0.004)$. PD-L1+ ILC had more diffuse PD-L1+ TIL (63\%) than did PD-L1- ILC $(23 \%)(\mathrm{p}=0.04)$. ER- ILC had more diffuse PD-L1+ TIL (50\%) than ER- ILC $(27 \%)(\mathrm{p}=0.03)$, and all cases of focal PD-L1+ TIL were ER+. PD-L1+ and PD-L1- ILC were equally ER+ (87-88\%) and ER- (12-13\%). A younger mean patient age was seen in tumors with a brisk TIL infiltrate (41 years) and in PD-L1+ ILC (51 years) than those with focal-moderate TIL ( 62 years, $\mathrm{p}=0.0001$ ) or PD-L1- ILC (61 years, $\mathrm{p}=0.02) .100 \%$ ILC in Black patients $(5 / 5)$ contained focal TIL infiltrate, compared to $45 \%$ (17/37) ILC in White patients $(\mathrm{p}=0.049)$. Only five $(10.6 \%)$ patients developed metastatic disease and four $(8.5 \%)$ patients died due to disease, with a median follow-up time of 44 months; the small numbers preclude survival analysis. Conclusions: A subset of primary breast ILC display brisk TIL infiltrates with high PD-L1 TIL, as well as PD-L1+ carcinoma cells. There was no correlation between TIL infiltrate density and carcinoma PD-L1 status and the ER status, HER2 status, or tumor grade of the lobular carcinomas. These results support exploring immunotherapy in both ER+ and ER- primary breast ILC.

294 Comparison of Estrogen Receptor Antibody Reagents Using Proficiency Testing Data

Megan Troxell, Jason L Hornick, Abiy B Ambaye, Kristin C Jensen, CAP Immunohistochemistry Committee. OHSU, Portland, OR; Brigham \& Women's, Boston, MA; UVM, Burlington, VT; VAPAHCS, Palo Alto, CA; CAP, Chicago, IL.

Background: Immunohistochemical analysis of estrogen receptor (ER) and progesterone receptor $(\mathrm{PgR})$ expression in breast cancer is the current standard of care and directly determines therapy. In $2010 \mathrm{ASCO} / \mathrm{CAP}$ published guidelines for ER and PgR predictive testing, encompassing pre-analytic, analytic, post-analytic factors, antibody validation and proficiency testing. Proficiency testing data allow comparison of different antibody clones among a large number of labs.

Design: The CAP PM2 survey utilizes tissue microarrays (TMA) of ten $2 \mathrm{~mm}$ cores per slide, with 20 ER and 20 PgR cores distributed twice a year. Beginning in 2014, all labs received unstained slides from the same TMA block for staining, scoring, and reporting. We analyzed survey data from 60 ER cores by antibody clone ( $N=1227-1388$ labs). Chi-Squared and Fisher exact analysis were used for pairwise comparisons between ER antibodies, with $\mathrm{p}<0.017$ considered significant.

Results: Laboratories employed the ER antibodies SP1-72\%; 6F11-17\%, 1D5-3\%; other- $7 \%$ in 2015 . While 41 of 60 ER cores were scored similarly by labs using each of the 3 antibodies, there were significant differences for others. Comparing 1D5 and SP1 results, 16 cores $(27 \%)$ had a statistically significant difference in the number of labs reporting a positive score, with more SP1+. Comparing 6F11 and SP1, 11 cores $(18 \%)$ produced a significantly greater number of SP1+ scores. For 1D5 and 6F11, 6 cores $(10 \%)$ had significantly different results. Strikingly, there were 4 cores scored negative by over half of the labs utilizing $1 \mathrm{D} 5$ or $6 \mathrm{~F} 11$, while SP1 produced mostly positive results (Table 1).

\begin{tabular}{|l|l|l|l|}
\hline TMA core & $1 \mathrm{D} 5+(\%)$ & $6 \mathrm{~F} 11+(\%)$ & $\mathrm{SP} 1+(\%)$ \\
\hline $14-05 \# 4$ & $8 / 61(13 \%)$ & $20 / 260(8 \%)$ & $892 / 957(93 \%)^{\wedge}$ \\
\hline $15-02 \# 3$ & $19 / 43(44 \%)$ & $75 / 252(30 \%)$ & $677 / 967(70 \%)^{*}$ \\
\hline $15-01 \# 10$ & $20 / 43(47 \%)^{\#}$ & $183 / 252(73 \%)$ & $890 / 970(92 \%)^{\wedge}$ \\
\hline $15-02 \# 7$ & $18 / 40(45 \%)$ & $134 / 243(55 \%)$ & $750 / 930(81 \%)^{\wedge}$ \\
\hline
\end{tabular}

^ $\mathrm{SP} 1$ vs $6 \mathrm{~F} 11$ and $\mathrm{SP} 1$ vs $1 \mathrm{D} 5 \mathrm{p}=0 ; * \mathrm{SP} 1$ vs $1 \mathrm{D} 5=0.0003, \mathrm{SP} 1$ vs $6 \mathrm{~F} 11=0$

"6F11 vs $1 \mathrm{D} 5 \mathrm{p}=0.0006$, not significant for other cores

Conclusions: Accurate ER and PgR testing in breast cancer is crucial to assignment of therapy. Prior studies have suggested increased sensitivity or found no difference in performance of the ER rabbit monoclonal antibody SP1, as compared to clones 1D5 and $6 \mathrm{~F} 11$. Our results from a large number of CAP-accredited labs demonstrate differences in staining results, with SP1 yielding more positive results.

295 Validation of the Rochester University Modified Magee Algorithm (RUMMA) for Risk Stratification of Estrogen Receptor Positive Breast Cancer Patients

Bradley Turner, Nyrie Soukiazian, Ping Tang, David G Hicks. Unversity of Rochester Medical Center, Rochester, NY; University of Rochester, Rochester, NY.

Background: Recently we published an algorithm [Turner et al. Mod Pathol. 2015 Jul;28(7)] based on clinicopathologic variables and risk prediction models developed at the University of Pittsburgh Magee Women's Hospital [Klein et al. Mod Pathol. 2013 May;26(5)]. We showed that this algorithm can provide similar risk information to the Oncotype DX test (Genomic Health, Redwood City, CA, USA), a multigene quantitative reverse transcription-polymerase chain reaction-based assay that estimates the risk of distant recurrence and predicts chemotherapy benefit for estrogen receptor (ER) positive breast cancer patients (BCP). We are now calling this algorithm the Rochester University Modified Magee Algorithm (RUMMA), and we have undertaken a clinical validation of the RUMMA by applying it to an independent set of ER+ BCP sent for Oncotype DX (ODX) testing.

Design: Using the RUMMA (abbreviated in figure 1), we risk stratified 153 consecutive cases with an available ODX recurrence score (ODXRS), identified from the pathology files at our institution between 2013 and 2015. We then correlated our results with the risk stratification categories used by the ODXRS [low $(<18)$, intermediate $(18-30)$, or high $(>30)]$. 


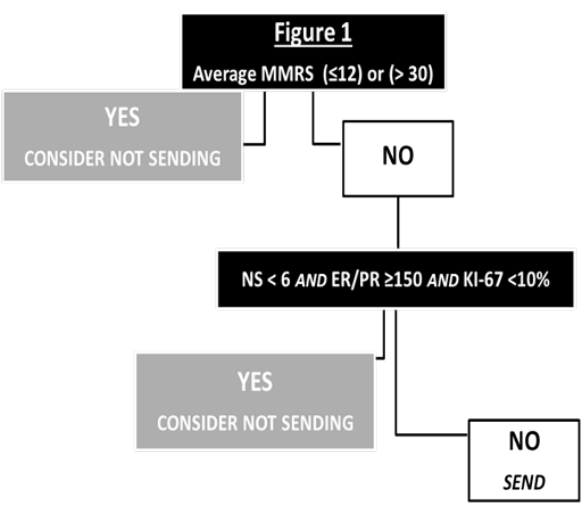

Results: $100 \%$ of cases that were predicted to be low and high risk by the RUMMA were also predicted to be low and high risk by the ODXRS (Table 1).

\begin{tabular}{|l|l|l|l|}
\hline \multirow{2}{*}{ Table 1 } & \multicolumn{3}{l|}{ ODXRS } \\
\cline { 2 - 4 } & HIGH & INTERMEDIATE & LOW \\
\hline MMRS $\leq 12$ & 0 & 0 & 14 \\
\hline NS $<6$, ER/PR $\geq 150$, KI- $67<10 \%$ & 0 & 0 & 11 \\
\hline MMRS $>30$ & 5 & 0 & 0 \\
\hline
\end{tabular}

$100 \%$ of cases with a high risk ODXRS $(\mathrm{n}=17)$ had an average Modified Magee recurrence score $($ MMRS $)>20.88 .6 \%$ of cases with a MMRS $<15(39 / 44)$ had a low risk ODXRS (ODXRS range $0-23$, mean ODXRS $=11.2$ ).

Conclusions: Preliminary results suggest that the RUMMA is a valid alternative to ODX testing for risk stratifying ER+ BCP into low and high risk of recurrence. RUMMA intermediate risk group cases should be considered for ODX testing, particularly if the MMRS is between 15 and 30 . We continue to identify additional cases to further test the RUMMA, and we are currently in the process of evaluating outcomes of patients to further qualify the clinical utility of the RUMMA.

296 Progesterone Receptor and Ki-67 Discordance in African American Women with Hormone Receptor Positive Breast Cancer Submitted for Oncotype DX Testing

Bradley Turner, Nyrie Soukiazian, Ping Tang, David G Hicks. University of Rochester Medical Center, Rochester, NY; University of Rochester, Rochester, NY.

Background: Although breast cancer (BC) incidence is lower among African American women (AA) compared to Caucasian American women (CA), AA have a 1.5-2.4 fold increased BC mortality risk, which persists after adjusting for stage, socioeconomic status, surgery, radiation treatment, and hormone receptor status. A higher incidence of estrogen receptor (ER) and progesterone receptor (PR) negative BC in AA patients compared with CA patients contributes in part to this increased $\mathrm{BC}$ mortality risk in AA women; however, relationships between hormone positivity and tumor proliferation may also possibly contribute to disparities in BC outcomes. Interest in the relationship between PR and Ki-67 has been increasing. We examine relationships between histologic variables and tumor proliferation in various ethnic groups with hormone positive $\mathrm{BC}$. Design: 394 consecutive BC cases with available ethnicity, Ki-67, PR, ER, Nottingham grade (NG), and Oncotype DX recurrence score (ODXRS) were identified from the pathology files at our institution between 2008 and 2015. 340 CA, 37 AA, 6 Latino women, and 6 Asian women were identified. We evaluated relationships between the modified PR H-score (mPRH), modified ER H-score (mERH), NG, Ki-67\%, and ODXRS.

Results: The mean Ki-67\% was 22.1 for all AA ( $\mathrm{n}=37)$, and 15.9 for all $\mathrm{CA}(\mathrm{n}=340)$. In cases with a Ki- $67 \geq 20$ (table), the ODXRS showed a significant difference between $\mathrm{AA}$ and $\mathrm{CA}$, with an unexpected lower ODXRS in AA $(\mathrm{p}=0.015)$. All correlations between pathologic factors showed the expected pattern, except for Ki-67 $\geq 20$ and PR. While Ki-67 $\geq 20$ showed the expected negative correlation with PR in the CA population, a positive correlation was present in the AA population.

\begin{tabular}{|l|l|l|}
\hline${ }^{*}$ mean & AA & CA \\
\hline Ki-67 $\geq 20(\mathrm{n})$ & $22 / 37(59.5 \%)$ & $105 / 340(30.9 \%)$ \\
\hline Ki-67 $\geq 20(\%) *$ & 32.0 & 33.1 \\
\hline mPRH $^{*}$ & 150.9 & 136.8 \\
\hline mERH $^{*}$ & 244.7 & 243.7 \\
\hline NG $^{*}$ & 7.1 & 7.2 \\
\hline ODXRS* & 18.2 & 22.9 \\
\hline
\end{tabular}

Conclusions: Preliminary results suggest the relationship between BC tumor proliferation and PR may be different in AA compared to CA. The ODXRS estimates risk of $\mathrm{BC}$ recurrence in hormone positive $\mathrm{BC}$ patients, and is heavily weighted on tumor proliferation and PR status. A positive correlation between PR and Ki- $67 \geq 20$ in AA, with a mean lower ODXRS compared to CA with a Ki-67 $\geq 20$, suggests that the ODXRS may underestimate the risk of $\mathrm{BC}$ recurrence in AA. We continue to identify additional cases, with clinical outcomes, to further test this hypothesis.
297 Expression of Cancer Stem Cell Markers Predicts Worse Outcome in Early Stage Triple Negative Invasive Breast Carcinoma

Shir-Hwa Ueng, Shin-Cheh Chen, Yu-Sun Chang, Yung-Chang Lin, Yun-Feng Lo, HsienKun Chang, Wen-Yu Chuang, Shih-Che Shen, Chuen Hsueh. Chang Gung Memorial Hospital, Linkou, Taoyuan, Taiwan; Chang Gung University, Taoyuan, Taiwan. Background: Triple negative invasive breast carcinoma (TNBC) is associated with aggressive behavior and worse prognosis. There is no standard chemotherapy regimen for TNBC. Cancer stem cells (CSC) have been described in various cancers. Their high tumorigenic capacity has been demonstrated in several studies. CSC are also thought to be more resistant to therapy, suggesting that the presence of CSC in tumors may determine treatment outcome. The identification of CSC could lead to future targeted therapies. The expression of CSC markers vary among breast cancer subtypes. However, CSC markers have not been extensively studied in TNBC. We aim to carry out such study in a large cohort of TNBC.

Design: 125 cases of TNBC (2005-2007) were collected from a single institution in Taiwan. Immunohistochemistry for ALDH1, EZH2, CD24, CD44, and CD133 was carried out on formalin-fixed paraffin embedded sections of mastectomy specimens. The results were correlated with age, tumor size, tumor grade, lymph node status, and TNM stage. The association of results with disease free survival (DFS) and overall survival (OS) was analyzed.

Results: No marker correlated with any clinicopathologic variable. Median follow up time was 100 months and maximum follow up time was 121 months. There were 92 (74\%) early stage (stage 1 and 2) patients and $33(26 \%)$ late stage (stage 3 ) patients. Only ALDH1 and EZH2 expression were found to be associated with survival of early stage patients. In total, the expression of ALDH1 and EZH2 was present in $66(53 \%)$ and $86(69 \%)$ tumors, respectively. Co-expression of ALDH1 and EZH2 was observed in $52(42 \%)$ tumors. In early stage tumors, the expression of ALDH1 and EZH2 was present in $48(53 \%)$ and $66(72 \%)$, respectively, and co-expression of both markers was present in $40(44 \%)$. Early stage patients whose tumors expressed ALDH1, EZH2 or both had shorter OS ( $\mathrm{P}=0.002, \mathrm{P}=0.031$, and $\mathrm{P}<0.001$, respectively) and $\mathrm{DFS}(\mathrm{P}=0.002$, $\mathrm{P}=0.016, \mathrm{P}<0.001$, respectively). No association of the markers with survival of late stage patients was found.

Conclusions: The expression of the CSC markers ALDH1 and EZH2 in early stage TNBC is associated with worse clinical outcome. The identification of CSC enriched TNBCs may provide further insight into CSC-targeted therapy.

\section{Similar Lymphocytic Infiltration Patterns in Primary Breast Cancer} and Corresponding Distant Metastases

Zsuzsanna Varga, Bettina Sobottka, Holger Moch. Institute of Surgical Pathology, Zurich, Switzerland.

Background: Primary breast cancer infiltrating lymphocytes (TIL) are acknowledged to correlate with disease free survival (DFS) in adjuvant and neoadjuvant settings. Little is known about the biology of metastasis infiltrating lymphocytes (MIL) although the local immunity of the metastatic site may critically influence the infiltrate composite. To address these questions we compared MIL with their matched TIL in 87 primary breast cancer tumors

and their corresponding distant metastases at four different anatomical locations.

Design: Sections of surgical specimens were immunohistochemically analyzed for $\mathrm{CD} 4, \mathrm{CD} 8$ and CD20 positive lymphocytes in three different tumor

compartments: intratumoral lymphocytes (iIL) defined as lymphocytes in direct contact with breast cancer cells, stromal lymphocytes (sIL) located within the intratumoral stromal tissue and invasive-margin lymphocytes (imIL).

Results: Overall we found fewer $(p<0.001)$ MIL than TIL. Within the tumor compartments imIL were more frequent than sIL and iIL, both in metastases and in the matched primary tumors $(p<0.001)$. CD4 $+\mathrm{T}$ cells were more numerous than $\mathrm{CD} 8+\mathrm{T}$ cells and $\mathrm{CD} 20+\mathrm{B}$ cells $(p<0.001)$. There was a similar pattern in primary tumors and their corresponding metastasis. Only patients with brain metastases differed from the others displaying less CD20+ B cells at the infiltrative margin of the primary tumor $(p<0.05)$.

Conclusions: In summary, IL were significantly reduced within metastases but still mirrored the infiltrate pattern of the primary tumor, interestingly, in all metastatic anatomical locations investigated. Our results suggest that the primary tumor may somehow assign the infiltrating lymphocyte pattern resumed at the metastatic sites.

299 Correlation of OncotypeDx Recurrence Score with IHC3/IHC4 and Grade in Ductal Carcinoma In Situ (DCIS)

Poonam Vohra, Gregor Krings, Manjiv Vohra, Yunn-Yi Chen. UCSF, San Francisco, CA. Background: Concern for overtreatment of DCIS triggers a need for biomarkers to identify patients with low $(\mathrm{L})$ or high(H) recurrence risk(RR). OncotypeDx DCIS Recurrence Score(RS) stratifies patients by RR and impacts clinical decisions but does not use HER2 status, and HER2+ DCIS may be more aggressive. IHC4, based on immunostaining(IHC) of estrogen(ER) and progesterone(PR) receptors, HER2 and Ki67, correlates with OncotypeDx RS in invasive tumors, but IHC4 or IHC3(lacking HER2) have not been evaluated in DCIS. In this study, we analyzed correlation of RS with IHC3/IHC4, HER2 and grade.

Design: ER, PR, HER2 and Ki67 IHC was evaluated in 36 DCIS cases with available RS. ER and PR were quantified by $\mathrm{H}$-score; HER2 by ASCO/CAP criteria as positive $(3+)$ or negative; Ki67 by percent. IHC3 and IHC4 were calculated as for invasive cancer. DCIS was graded by CAP(I, II, III) and Van Nuys criteria(L vs combined intermediate $[\mathrm{I}] / \mathrm{H}]$ ).Analysis was performed with and without $\mathrm{HER} 2+$ cases.

Results: Patient age ranged from 34-74 years. 32 cases were ER+ and $25 \mathrm{PR}+$. H-scores ranged from $0-300$ and Ki67 from 1.2-14.7. HER2 was $3+$ in $8,2+$ in 3 and $0-1+$ in 25 cases. Most(6/8) HER2+(including 2 ER-/PR-) cases had L(4) or I(2) RS. Correlation 
between $\mathrm{RS}$ and IHC3/IHC4 was poor if including HER $2+\operatorname{cases}\left(\mathrm{R}^{2}=.28\right.$ and .15$)$ but good if excluding HER2+ cases(IHC3 $\left.\mathrm{R}^{2}=.73\right)$. RS also correlated better with grade if HER2+ DCIS were excluded. Of HER2- cases, 22/24(92\%) grade I/II DCIS had L/I RS and 13/15(87\%) Van Nuys L grade DCIS had L RS. No correlation was seen between RS and Ki67 or ER/PR.

\section{Correlation of IHC3 with RS in DCIS (HER2+ and HER2-)}
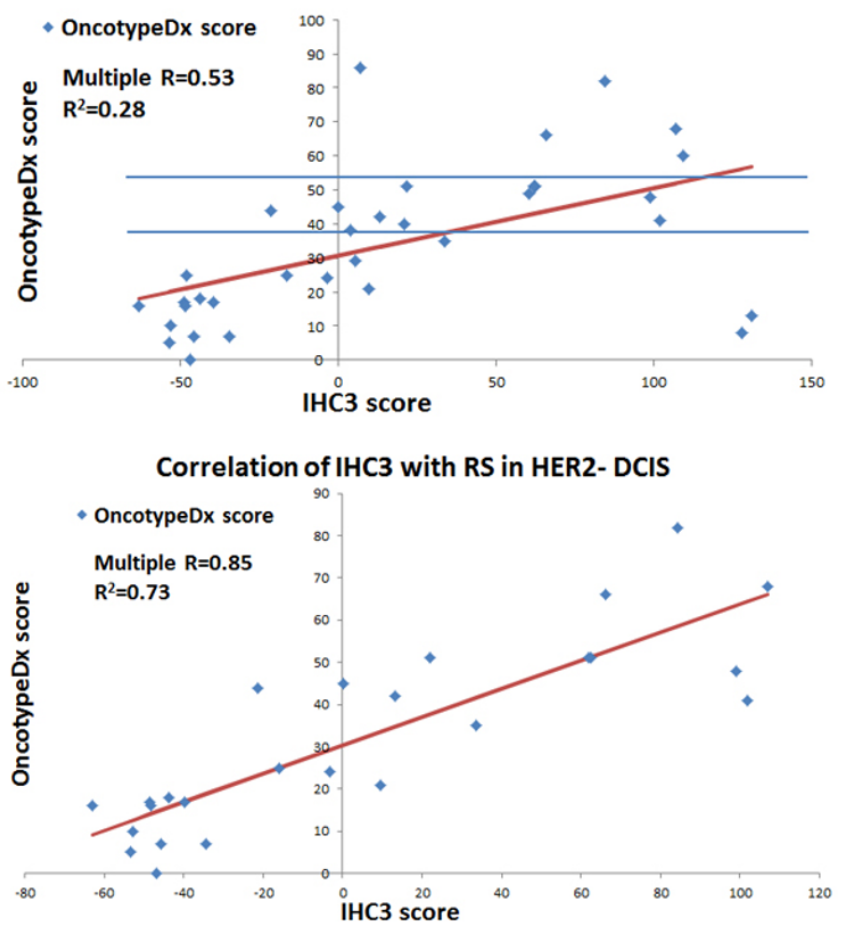

\begin{tabular}{|l|l|l|l|l|}
\hline \multirow{2}{*}{ Grade } & \multicolumn{2}{|l|}{ RR Group } \\
\cline { 3 - 5 } & L $(<39)$ & $\mathrm{I}(39-54)$ & $\mathrm{H}(>54)$ \\
\hline \multirow{3}{*}{ Van Nuys } & L & 13 & 1 & 1 \\
\cline { 2 - 5 } & I/H & 4 & 7 & 2 \\
\hline \multirow{3}{*}{ CAP } & I & 2 & 0 & 0 \\
\cline { 2 - 5 } & II & 15 & 5 & 2 \\
\cline { 2 - 5 } & III & 0 & 3 & 1 \\
\hline
\end{tabular}

Conclusions: DCIS RS may be low in HER2+ DCIS, which is known to be aggressive. HER2 IHC testing should be considered in grade II/III or Van Nuys combined I/H grade DCIS with low RS to avoid undertreatment. IHC3 correlates with RS in HER2- DCIS and requires validation in larger series.

\section{PD-L1 Protein Expression and PD-L1 Gene Amplification in Breast}

\section{Cancer}

Olga Voronel, Siddhartha Dalvi, Bhaskar VS Kallakury, Christine E Sheehan, Ann B Boguniewicz, Jeffrey S Ross. Albany Medical College, Albany, NY; Georgetown University Hospital, Washington, DC

Background: PD-L1 (programmed death - ligand 1)/CD274, is a transmembrane protein that interacts with PD-1 receptor. Interaction of PD-L1 on tumor cells with PD-1 on tumor-infiltrating cytotoxic CD8+ T-cells induces inactivation and apoptosis of T-cells, leading to tumor evasion of the immune system. Inhibition of PD-L1 by immune checkpoint inhibitory drugs has been effective in numerous clinical trials, but PD-L1 expression has not been widely studied in breast cancer (BC).

Design: Formalin-fixed, paraffin-embedded sections from 84 invasive BCs [71 ductal (IDC), 13 lobular (ILC)] were immunostained (Ventana) using rabbit monoclonal PD-L1/CD274 (Spring Bio, clone SP142). Membranous PD-L1 immunoreactivity was scored as negative, low $(1-24 \%)$ and high $(=$ or $>25 \%)$ positive in tumor cells and tumor infiltrating lymphocytes (TILs). PD-L1 (CD274) gene amplification was determined by hybrid capture based comprehensive genomic profiling (CGP) on a separate cohort of 6,751 BCs. Results were correlated with clinicopathologic variables. Results: PD-L1 immunoreactivity was noted as low positive in $4(5 \%)$ tumors [2 tumor+/TIL+ and 2 tumor+/TIL-], and low positive in TILs in $5(6 \%)$ additional cases [tumor-/TIL+]; all $9(100 \%)$ were IDCs. No PD-L1 immunoreactivity was noted in the TILs in the remaining cases, despite the presence of prominent TILs in a majority of cases. PD-L1 protein expression correlated with overall survival [100\% PD-L1 positive cases and $0 \%$ tumor- $/ \mathrm{TIL}+$ cases expired, $(\mathrm{p}=0.002)]$, while showing a trend toward correlation with tumors that did not recur $[100 \%$ PD-L1 positive cases and $100 \%$ tumor- $-\mathrm{TIL}+$ cases did not recur, $(\mathrm{p}=0.08)]$, and with age at diagnosis in the $\mathrm{ER}+$ subgroup $[100 \%$ PD-L1 positive cases diagnosed at a peri-menopausal age and $100 \%$ tumor-/TIL+ cases diagnosed at a post menopausal age, $(\mathrm{p}=0.069)$. PD-L1 (CD274) gene amplification was identified in only $57(0.1 \%)$ of 6,751 BCs submitted for CGP. On multivariate analysis, tumor grade $(\mathrm{p}=0.025)$ and disease recurrence $(\mathrm{p}<0.0001)$ independently predicted overall survival.

Conclusions: Five percent of BCs showed PD-L1 immunoreactivity and an additional $6 \%$ had immunoreactivity within the TIL component only. CGP of an additional 6,751 breast cancers showed $P D-L 1$ gene amplification in only $0.1 \%$ of cases. Similar to other reports, we found that PD-L1 protein expression was associated with lower overall survival as all $(100 \%)$ patients with PD-L1 positive tumors expired. Our findings support continued exploration of anti-PD-L1 agents as potential therapies for this small subset of aggressive $\mathrm{BC}$.

\section{1 "Triple Negative" Breast Cancers vs. "Quadruple Negative" Breast} Cancers: The Androgen Receptor Factor

Elena Vrotsos, Carmen Gomez-Fernandez, Mahsa Khanlari, Mehrdad Nadji. University of Miami, Miami, FL.

Background: Triple negative breast cancers (TNBC) are unresponsive to traditional endocrine and HER2 targeted therapies. Furthermore, there are no current effective target-directed therapeutic approaches for TNBC. Recent demonstration of androgen receptor (AR) in a number of TNBC has renewed the interest in hormonal therapy regimens targeting $\mathrm{AR}$.

However, since not all triple negative breast cancers express AR, we set out to study AR expression in a cohort of TNBC with special emphasis on

immunohistochemically confirmed histologic subtypes.

Design: Ninety-three consecutive TNBC were retrieved from the files of the Department of Pathology at the University of Miami, Jackson Memorial Hospital. On the basis of histomorphology and/or immunohistochemical profiles they were further classified into medullary, metaplastic, apocrine, lobular and invasive ductal carcinoma of no special type (IDC-NST). The expression of AR was semi quantitatively scored following ASCO/ CAP recommendations for ER scoring.

Results: Out of the 93 cases, 51 were classified as IDC-NST, 14 as medullary, 12 as apocrine, 11 as metaplastic, and 5 as lobular carcinoma.

Out of 93 cases $39(42 \%)$ were positive for AR, including all $12(100 \%)$ apocrine, 4 $(80 \%)$ lobular and $23(45 \%)$ IDC-NST. None of the medullary or metaplastic carcinomas expressed AR.

Conclusions: TNBC comprise a heterogeneous group of breast cancers that in addition to IDC-NST, includes apocrine, lobular, medullary, and metaplastic carcinomas. The expression of AR varies among these histologic subtypes. All apocrine and most lobular "triple negative" breast carcinomas express AR and may potentially benefit from antiandrogen therapy, while medullary and metaplastic breast carcinomas are negative for AR. This latter AR-negative subset of TNBC could be henceforth reclassified as "quadruple negative" breast cancers.

302 Comparison of Magee and OncotypeDx Recurrence Scores in Luminal-B Immunophenotype Breast Cancers

Ann E Walts, Shikha Bose. Cedars-Sinai Medical Center, Los Angeles, CA. Background: Oncotype DX is currently used to estimate the risk of distant recurrence and predict chemotherapy benefit in estrogen receptor positive (ER+) breast cancer. Recent reports suggest that the Magee recurrence scores obtained using data in a pathology report can predict the OncotypeDX recurrence score (OncoRS) and obviate ordering the expensive assay in a substantial number of ER+ breast cancers.

Design: Tumor size, Nottingham score, and ER, PR, Ki67, and Her 2 information were extracted from pathology reports of 100 consecutive ER+ invasive breast cancers with Ki67 $\geq 14 \%$ and available OncoRS. Data were entered into the automatic RS calculator accessible at the Univ. of Pittsburgh Dept. of Pathology website to obtain the recurrence scores for Magee equations \#1-\#3 (Klein et al. Mod Pathol 2012). As all cases were evaluated for Ki67, the average of Magee equations \#1 and \#3 was calculated as the "average modified Magee recurrence score" (AMM RS). The AMM RS and OncoRS were compared per tumor. Follow-up (2-90 months; median 14 mos.) was obtained from our hospital's cancer registry.

Results: Median age at lumpectomy was 56 years (27-78 yrs) and median tumor size was $1.6 \mathrm{~cm}(0.7-7.0 \mathrm{~cm})$. OncoRS were 44 low-risk (LR), 39 intermediate-risk (IntR), and 17 high-risk (HR). AMM RS were $42 \mathrm{LR}, 50 \mathrm{IntR}$, and 8 HR. The table shows the distribution and concordance of OncoRS and AMM RS. 58 tumors (28 LR, 24 IntR, 6 $\mathrm{HR}$ ) had concordant scores including the 2 tumors with distant recurrences: 1 with HR scores and a lung metastasis at 25 mos. and 1 with IntR scores and a bone metastasis at 53 mos. post diagnosis. 42 tumors had discordant scores (29 lowR/IntR, $12 \mathrm{IntR} /$ HR, 1 LR/HR). The patient with the LR (by OncoRS)/HR (by AMM RS) discordance is disease free at 14 mos. post diagnosis.

Comparison of AMM RS and OncoRS $(\mathrm{N}=100)$

\begin{tabular}{l|c|c|c}
\hline AMM RS & Low-risk OncoRS & Intermediate-risk OncoRS & High-risk OncoRS \\
\hline & & & \\
\hline Low-risk $(\mathbf{n}=\mathbf{4 2})$ & 28 & 14 & 0 \\
\hline Intermediate-risk $(\mathbf{n}=\mathbf{5 0})$ & 15 & 24 & 11 \\
\hline High-risk $(\mathbf{n}=\mathbf{8})$ & & & 6
\end{tabular}

Conclusions: OncoRS and AMM RS were concordant in 58\% of our study cohort. Only 1 case showed a 2-step (LR/HR) discordance. Given that recurrence scores impact treatment, the high percentages of tumors with IntR scores (50\% AMM RS and 39\% OncoRS) is an important limitation of both recurrence scoring algorithms. The low distant recurrence rate $(2 \%)$ to date suggests larger prospective studies with long-term follow-up are needed to determine which scoring system serves patients best. 
303 TP53 Alteration in Morphologically Normal/Benign Cells in Breast Terminal Ductal Lobular Units (TDLU) in Patients with or without BRCA Germline Mutation: A Marker for Early Detection of High Risk Patients? Xi Wang, Amber A El-Halaby, Todd S Laughlin, Paul G Rothberg, Kristin Skinner, David G Hicks. University of Rochester, Rochester, NY.

Background: Germ-line mutations in BRCA genes have been shown to predispose patients to breast cancer. Studies have suggested that p53 alteration is a necessary step in tumorigenesis in BRCA carriers.

Design: We retrospectively analyzed the TP53 status by immunohistochemistry (IHC) in the normal/benign breast TDLUs of 19 patients who had BRCA genes germline mutation and breast cancer (13 HG, 6 non-HG), 30 BRCA carrier patients who had bilateral prophylactic mastectomy, 157 regular breast cancer patients (106 HG, 51 non$\mathrm{HG}$ )and 50 patients with mammoplasty. At least two tissue blocks were selected from each breast specimen. P53 was read as positive if $>50 \%$ of the tumor cell nuclei were stained, and more than 10 consecutive normal/benign cell nuclei were stained. TP53 gene sequencing was performed on selected tumors with inconclusive p 53 staining. Further ER and Ki67 IHC stains were performed on the blocks with p53 positive normal/benign TDLU.

Results: Six of the $19(32 \%)$ breast carcinomas in BRCA patients were positive for p53, all in igh grade tumors $(6 / 13,46 \%) ; 3$ of the 6 cases were also focally $p 53$ positive in adjacent normal/benign TDLU. Fifty three of the 157 (36\%) regular breast carcinomas had TP53 alteration, 48 in HG tumors (45\%), 5 in non-HG tumors (9.8\%); 19 of the 53 cases were also focally p 53 positive in adjacent normal/benign TDLU (17 in HG cases, 2 in non-HG cases). Four of the $30(13 \%)$ cases with bilateral prophylactic mastectomies in BRCA carriers had focal p53 positivity in normal/benign TDLU in at least unilateral breast, with one in bilateral breasts, compared with none $(0 \%)$ in mammoplasty specimens. The $\mathrm{p} 53$ positive normal/benign cells were ER negative, and did not show an increased Ki67 labeling index. The morphology of these cells appeared to be with slightly enlarged and hyperchromatic nuclei and prominent nucleoli, but still within the limits of normal/benign cells.

Conclusions: TP53 is altered in normal/benign appearing breast TDLUs in BRCA carriers and in patients with $\mathrm{HG}$ breast carcinomas, indicating an early event in breast carcinogenesis. These cells are ER negative, the same as the HG carcinoma, but remain inactive with low Ki67 labeling index, indicating further genetic changes are needed to trigger the unlimited tumor growth. Their special morphology and IHC positivity for p53 may serve as the "p53 signature", an indicator for future potential risk of TP53 positive high grade breast carcinoma, especially for BRCA carriers.

304 Distinctions in Breast Tumor Recurrence Patterns Post-Therapy among Ethnically Distinct Populations

Nikita Wright, Jun Xia, Remus Osan, Pranay Neema, Dora II'yasova, Sergey Klimov, Mildred Jones, Padmashree CG Rida, Guilherme Cantuaria, Ritu Aneja. Georgia State University, Atlanta, GA; Northside Hospital Cancer Institute, Atlanta, GA; Northside Hospital Cancer Data Center Institute, Atlanta, GA.

Background: Clinical studies have revealed higher risk of tumor recurrence in AA patients compared to EA patients, presumably contributing to the inequality in clinical outcomes among the ethnic groups. However, distinctions in recurrence patterns posttherapy between the ethnicities remain poorly characterized.

Design: In this clinical study, we compared rates (per 1000 person-year) and patterns of tumor recurrence post-therapy between AA $(n=1850)$ and EA breast cancer patients $(n=7931)$ from a large cohort of breast cancer patients $(n=10,504)$ treated from 20052015 at Northside Hospital in Atlanta, Georgia.

Results: We observed higher rates of tumor recurrence (21.42 vs.13.28) in AA ( $\mathrm{n}=47)$ compared to EA breast cancer patients $(\mathrm{n}=160)(\mathrm{HR}=1.676$; CI: 1.21-2.323) and recurrence rates remained higher after controlling for age, grade, and stage $(\mathrm{HR}=1.532$; CI: 1.025-2.291). Moreover, we discerned stronger inclinations in AA patients toward regional tumor recurrences (HR: 2.05; CI: 1.0-4.24) and higher rates of distant tumor recurrences (11.85 vs. 6.72) compared to EA patients (HR: 1.76; CI: 1.13-2.74). Additionally, stronger tendencies toward distant recurrence to multiple sites in AA patients compared to EA patients were observed (1.37 vs. 0.91). Inversely, rates of local tumor recurrences were not statistically higher in AA compared to EA patients (5.01 vs. 4.07) (HR: 1.19; CI: 0.62-2.29). Similar trends were maintained after patients underwent lymph node surgery, radiation, hormone, and adjuvant therapy. Interestingly, among patients that received neo-adjuvant chemotherapy, AA exhibited a lower propensity toward tumor recurrence than EA (19.77 vs. 28.95). Furthermore, AA patients that received neoadjuvant chemotherapy displayed higher inclinations toward local tumor recurrences (13.18 vs. 1.81) and lower inclinations toward regional ( 0 vs. 6.39$)$ and distant tumor recurrences (6.59 vs. 20.81).

Conclusions: Our results demonstrate higher incidence rates of tumor recurrence and more severe tumor recurrence patterns post-therapy in AA compared to EA breast cancer patients. Interestingly, we observed attenuation of these aggressive recurrence patterns in AA patients who received neoadjuvant chemotherapy. This data suggest that this is the first clinical study to suggest that chemotherapy improves breast cancer recurrence rates and patterns in AA patients and may theoretically diminish the observed racial divide in tumor recurrence risk.
305 Clinical-Pathologic Analysis of Luminal B Breast Carcinoma

Xuemei Wu, Judy C Boughey, Ann M Moyer, Krishna R Kalari, Vera J Suman, Jason P Sinnwell, Erin E Carlson, Travis J Dockter, Alvaro Moreno-Aspitia, Richard J Gray, Sarah A McLaughlin, Donald W Northfelt, Katie N Jones, Amy L Conners, Eric D Wieben, Liewei Wang, Richard M Weinshilboum, Matthew Goetz, Daniel W Visscher. Mayo Clinic, Rochester, MN; Mayo Clinic, Jacksonville, FL; Mayo Clinic, Scottsdale, AZ.

Background: Breast carcinomas are increasingly classified by intrinsic molecular subtypes to determine their clinical management. However, optimal methods for subtyping have yet to be determined and their correlation with clinical conventional histological features has not been well studied, particularly for the luminal B subtype. Design: Diagnostic breast biopsies from women enrolled in the Breast Cancer GenomeGuided Therapy (BEAUTY) study, a neoadjuvant chemotherapy study using weekly paclitaxel followed by doxorubicin/cyclophosphamide, were reviewed to identify luminal B tumors as defined by immunohistochemical (IHC) staining criteria (ER and/ or $\mathrm{PR} \geq 10 \%$, HER 2 negative, Ki67 $\geq 15 \%$ ). We evaluted this group for concordance with PAM50 subtyping, and summarized their characteristic morphologic features and response to chemotherapy.

Results: The 22 luminal B breast cancers showed 2 main histological patterns. The "classic" morphology $(\mathrm{n}=16)$ demonstrated tumor cells arranged in non-ductule-forming discrete nests surrounded by retracted desmoplastic stroma with hyaline collagenous central scarring. Lymphoid infiltration and DCIS were minimal. The second pattern consisted of lobular carcinoma with high-grade nuclei and apocrine cytoplasm $(n=5)$. One case showed clear cell/columnar cytology. PAM50 classification matched the IHC designation in 11 of 22 cases (50.0\%). Of the "non-luminal B" tumors by PAM50, 6 of "classic" histologic type were classified as luminal A and 2 as basal type, while one of "lobular" type was classified as basal type and 2 as normal type. Most luminal B tumors ( 19 of $22,86.4 \%$ ) showed high residual cancer burden at surgery; however, of the 21 cases with Ki67 available, most demonstrated reduction in proliferation with Ki67<15\% at surgery ( 18 of $21,85.71 \%$ ).

\begin{tabular}{|c|c|c|c|c|c|}
\hline \multirow{2}{*}{$\begin{array}{c}\text { Luminal B (By IHC) } \\
\text { Morphology }\end{array}$} & \multicolumn{4}{|c|}{ PAM50 Subtyping } & Agreement \\
\cline { 2 - 5 } & Lum A & Lum B & Basal-like & Normal & \\
\hline "Classic" (n=16) & 6 & 8 & 2 & 0 & 50 \\
\hline "Lobular" (n=5) & 0 & 2 & 1 & 2 & 40 \\
\hline "Clear cell” (n=1) & 0 & 1 & 0 & 0 & 100 \\
\hline
\end{tabular}

Conclusions: Luminal B breast tumors (by IHC) are characterized by a limited spectrum of histological patterns. IHC algorithms for luminal B subtyping result in significant discrepancy with gene expression assays, potentially reflecting complex biology that cannot be fully characterized by a small number of markers. Luminal B tumors showed a low pathologic complete response rate, but the proliferation rate in residual tumor was reduced compared to baseline.

306 SoX10 Is a Novel Sensitive Diagnostic Marker for Adenoid Cystic Carcinoma of the Breast: Immunohistochemical Analysis of Adenoid Cystic Carcinoma

Chen Yang, Lingxin Zhang, Souzan Sanati. Washington University School of Medicine, St. Louis, MO.

Background: Adenoid cystic carcinoma (AdCC) of the breast is a rare triple-negative breast cancer with favorable prognosis. Given the low prevalence of this tumor, its defining features are not well established. The immunophenotypic features of AdCC of the breast remains unclear except for the fact that they are commonly triple negative (ER, PR, and Her-2 negative). SRY-related HMG-box 10 (SOX10) protein is a transcription factor known to be crucial in the specification of the neural crest and maintenance of Schwann cells and melanocytes. Expression of SOX10 as a diagnostic marker has been previously established in salivary gland AdCC and basal-like breast carcinoma; however, SOX-10 has not been studied in primary breast AdCC. Here, we examined SOX10 expression in AdCCs from various anatomic sites including breast, and correlated with other markers to investigate the histogenesis of this tumor.

Design: A total of 11 cases of breast AdCC with formalin-fixed paraffin-embedded (FFPE) blocks were obtained from archives. 17 age-matched salivary gland AdCCs and 5 metastatic AdCCs were also retrieved. These cases were centrally reviewed by three pathologists with confirmation of the diagnosis. Tissue microarrays of $3 \mathrm{~mm}$ cores of each case were made in triplicates. The expression of SOX10, Ki-67, c-kit, beta-catenin, EMA, p63, CK7, and CK5/6 were assessed by immunohistochemistry. Results: All cases of breast (100\%) and metastatic (100\%) AdCCs showed diffuse nuclear positivity for SOX10. Except one case which showed loss of beta-catenin expression and developed subsequent metastasis to the T10 vertebra, all cases showed diffuse membrane beta-catenin expression. No significant difference in expression of CK7, p63, CK5/6, C-kit, EMA and Ki-67 was noted. Table 1 summarizes the results. 


\begin{tabular}{|l|l|l|l|}
\hline & Breast AdCC & Salivary AdCC & Metastatic AdCC \\
\hline SOX10 & $93 \%(63-100 \%)$ & $83 \%(19-100 \%)$ & $96 \%(75-100 \%)$ \\
\hline beta-catenin & $100 \%$ & $94 \%(30-100 \%)$ & $100 \%$ \\
\hline CK7 & $73 \%(15-100 \%)$ & $82 \%(6-100 \%)$ & $81 \%(25-100 \%)$ \\
\hline CK5 $/ 6$ & $66 \%(0-100 \%)$ & $79 \%(50-100 \%)$ & $50 \%(5-65 \%)$ \\
\hline p63 & $93 \%(2-88 \%)$ & $64 \%(1-97 \%)$ & $50 \%(2-77 \%)$ \\
\hline EMA & $3 \%(0-17 \%)$ & $12 \%(1-52 \%)$ & $34 \%(1-93 \%)$ \\
\hline C-kit & $68 \%(25-100 \%)$ & $61 \%(13-100 \%)$ & $50 \%(15-87 \%)$ \\
\hline Ki-67 & $7 \%(1-20 \%)$ & $6 \%(1-27 \%)$ & $12 \%(5-25 \%)$ \\
\hline
\end{tabular}

309 The Forkhead Box Transcription Factor FOXC1 Further Stratifies Prognosis of Patients with Triple-Negative Breast Cancer

Yong Yue, Kristine Astvatsaturyan, Xiaojiang Cui, Xiao Zhang, Benedick Fraass, Shikha Bose. Cedars-Sinai Medical Center, Los Angeles, CA.

Background: Triple-negative breast cancer (TNBC) is a highly diverse group of aggressive cancers with poor survival. Recent studies suggest that the FOXC1 transcription factor is a critical regulator of EGFR function in basal-like breast cancer, and may allow better stratification of risks in TNBC patients.

Design: 126 consecutive stage I-III TNBCs were retrieved from 2008 to 2012. All patients had lumpectomy or radical mastectomy as primary treatment. IHC staining for FOXC1, EGFR, CK5/6 and Ki-67 was evaluated on whole sections. Disease-freesurvival (DFS) was correlated with expression of biomarkers, and clinicopathological variables including age, tumor size, grade and lymph node status. The prognostic values of variables were determined by univariate and multivariate Cox analysis. Patients were stratified into risk groups using Kaplan-Meier curves and log-rank tests.

Conclusions: Similar to AdCC of salivary gland, SOX10 is a sensitive marke histogenesis of AdCC of breast and its cell origin.

307 Evaluation of Histopathologic Features That Affect Interobserver Agreement on Tumor Infiltrating Lymphocyte (TIL) Quantities in Breast Cancer

Fei Yang, Shannon Swisher, Elizabeth A Mittendorf, Genevieve R Lyons, Coya Tapia, Xiuhong Wang, Roland L Bassett, Kelly K Hunt, Aysegul A Sahin, Yun Wu. The University of Texas MD Anderson Cancer Center, Houston, TX.

Background: Recent studies have shown that TILs are associated with clinical outcome of patients with breast cancer and serve as a marker for predicting the efficacy of chemotherapy and anti-HER2 therapy in triple-negative and HER2-enriched breast cancer. An International TILs Working Group has published consensus recommendations for standardization of TIL evaluation in breast cancer. Using the proposed methodology, we have recently shown moderate and substantial interobserver agreements among 4 pathologists for the evaluation of stromal TILs (sTILs) and intraepithelial TILs (iTILs) (kappa $=0.57$ and 0.61 , respectively). The aim of the current study was to determine whether specific histopathologic features contribute to the lack of better agreement. Design: TILs were assessed on H\&E-stained slides obtained from core biopsies from 75 patients with triple-negative breast cancer. To minimize selection bias, the core biopsies to be evaluated for each patient were selected by a single pathologist. Four pathologists independently reviewed each core biopsy. sTILs, defined as mononuclear cells within the stroma not in direct contact with tumor cells, were evaluated and categorized as constituting $<10 \%, 10-50 \%$, or $>50 \%$ of the stromal area. Cases lacking agreement between at least 3 of the 4 pathologists were reviewed and discussed by the entire group to determine whether specific pathologic features contributed to the lack of agreement. Immunohistochemical staining for cytokeratin and CD45 was performed in select cases. Results: In $10(13 \%)$ cases, there was not agreement between at least 3 of the 4 pathologists. The following histopathologic features contributed to the difficulty in enumerating TILs: widespread tumor necrosis, marked individual cell necrosis (apoptosis), the presence of reactive plasma cells mimicking tumor cells, and plasmatoid tumor cells mimicking infiltrating plasma cells. Additional staining with anti-cytokeratin (epithelial marker) and anti-CD45 (pan-leukocyte marker) antibodies was able to identify questionable cells as either epithelial or inflammatory.

Conclusions: Although application of the consensus statement methodology results in moderate agreement between pathologists in the quantification of TILs, there are specific pathologic features that contribute to the difficulty in TIL enumeration. Especially in tumors with extensive apoptotic bodies and prominent plasmocytic infiltrate, immunohistochemical staining is recommended for more accurate evaluation of TILs.

308 Down-Regulation of Fructose-1,6-Bisphosphatase (FBP1) in TripleNegative Breast Cancer

Esther Yoon, Kenneth Hennrick, Yong Mao, Konstantin Volyanskyy, Nevenka Dimitrova, John T Fallon, Minhao Zhong. New York Medical College, Valhalla, NY; Philips, 345 Scarborough Road, NY.

Background: Triple-negative breast cancer (TNBC) is about $15-25 \%$ of all breast cancer (BC) with relatively aggressive behavior. Due to heterogeneity and poor understanding, there is no targeted therapy available. Recently, proving cancer metabolic advantage through loss of FBP1 has been associated with several types of cancer. Therefore, we want to investigate the possible association of loss FBP1 and different subtypes of BC. Design: Our study composed two sections: in-silico analysis of transcriptome data and confirmation of immunohistochemistry (IHC) stain for FBP1. Level III RNA-seq data from The Cancer Genome Atlas (TCGA) 994 BC samples were downloaded and analyzed. In each sample, gene expression of ER, PR, Her-2 and FBP1 were normalized to those from pooled benign breast tissue. Then, we categorized these samples into luminal A, luminal B, Her2 type and triple negative. We also performed FBP1 IHC stain on $33 \mathrm{BC}$ samples which have been previously well documented with ER, PR and Her2 status by IHC stains.

Results: In 994 TCGA breast cancer samples, there are 367 luminal A, 337 luminal B, 198 Her 2 type and 92 triple negative with average relative FBP1 expression 1.17, $1.46,-0.63$ and -1.27 respectively. Among 33 cases of $\mathrm{BC}$ cases, there is 20 luminal A, 6 luminal B, 1 Her2 type and 6 triple negative based on IHC stains. Only TNBC showed complete loss of FBP1. All other subtype BC and benign glandular component are positive for FBP1.

Conclusions: Loss of FBP1 strongly associated with TNBC, but not other subtype of BC. FBP1 IHC stain could be used to separate TNBC from other subtype of BC. Targeting FBP1 signaling pathway could be a potential therapy strategy.

Results: Median age was 58 years (range, 28-92 years), and a median DFS was 9.7 months. Multivariate Cox analysis identified three significant variables: tumor stage $(\mathrm{p}=0.002)$, EGFR $(\mathrm{p}=0.043)$, and FOXC1 $(\mathrm{p}=0.051)$. EGFR stratified TNBCs into low and high risk groups based on percent positivity of tumor cells, and FOXC1 further stratified each EGFR risk groups. In the low EGFR group, low FOXC1 had significantly better survival than high $\mathrm{FOXC1}(100 \%$ vs $61 \%$ at 50 months). For patients with high EGFR, no significant survival difference was observed between high and low FOXC1 $(63 \%$ vs $58 \%)$. The risk stratification with integrative EGFR and FOXC1 was statistically significant with log-rank $\mathrm{p}=0.049$.

\begin{tabular}{|l|l|l|l|l|l|l|}
\hline $\begin{array}{l}\text { Risk } \\
\text { groups } \\
(\mathrm{n}=126)\end{array}$ & $\begin{array}{l}\text { Patient } \\
\text { Number } \\
(\%)\end{array}$ & $\begin{array}{l}\text { Median } \\
\text { DFS } \\
(\text { Months })\end{array}$ & $\begin{array}{l}\text { Events in the } \\
\text { Group (\%) }\end{array}$ & $\begin{array}{l}\text { Median } \\
\text { EGFR (\%) }\end{array}$ & $\begin{array}{l}\text { Median } \\
\text { Foxc1\% } \\
(\%)\end{array}$ & $\begin{array}{l}\text { Median } \\
\text { CK5/6 (\%) }\end{array}$ \\
\hline 1 & $17(13 \%)$ & 16.6 & $0(0 \%)$ & 2.0 & 21.0 & 0.0 \\
\hline 2 & $25(20 \%)$ & 16.0 & $4(16.0 \%)$ & 5.0 & 74.0 & 5.0 \\
\hline 3 & $30(24 \%)$ & 11.3 & $7(23.3 \%)$ & 75.0 & 11.0 & 7.5 \\
\hline 4 & $54(43 \%)$ & 6.4 & $16(29.6 \%)$ & 60.0 & 76.0 & 20.0 \\
\hline
\end{tabular}

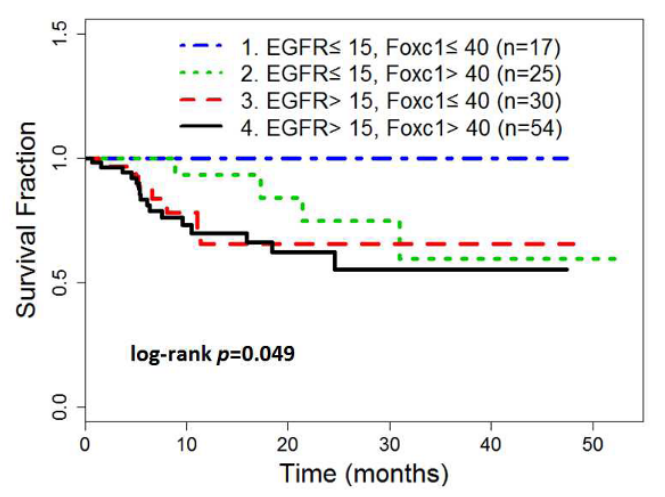

Figure 1. Kaplan Meier curves of DFS of risk groups for TNBC patients. The patients were first stratified into low and high EGFR risk groups ( $\leq 15 \%$ vs $>15 \%)$. The two risk groups were further stratified by FOXC1 $(\leq 40 \%$ vs $>40 \%)$. Group $1(n=17)$ with low expressions of EGFR and FOXC1 had significant better prognosis than other risk groups.

Conclusions: FOXC1 expression improves the predictive accuracy of survival, and can be used to further stratify risks in TNBCs. TNBC patients with low EGFR and low FOXC1 expressions may benefit from less aggressive therapy.

\section{Mucocele-Like Lesions Diagnosed on Breast Core Biopsy and the} Risk of Upgrade to Carcinoma on Excision

Gloria Zhang, Dana Ataya, Paulette Lebda, Benjamin C Calhoun. Cleveland Clinic, Cleveland, $\mathrm{OH}$.

Background: Mucocele-like lesions (MLLs) of the breast are characterized by mucinfilled cysts and extrusion of mucin into the adjacent stroma. Most MLL diagnosed on core biopsy are excised to exclude the possibility of a limited sampling of an invasive mucinous carcinoma. However, the data on the reliability of the diagnosis of MLL on core biopsy are limited. Our goal was to review the pathologic and radiologic features of core biopsies with MLL to determine if all patients should undergo immediate surgical excision.

Design: Cases were identified by searching the anatomic pathology information system for "mucocele" and "mucin" in breast core biopsy reports. Patients with co-existing invasive carcinoma or ductal carcinoma in situ in the same core biopsy were excluded. The pathology and radiology were reviewed by dedicated breast pathologists and breast radiologists.

Results: We identified 32 patients with MLL on core biopsy with an average age of 56 years (range 27-81). The indication for core biopsy was calcifications in $23(72 \%)$ and a mass or density in $9(28 \%)$. There were $26(81 \%)$ stereotactic core biopsies and 6 $(19 \%)$ ultrasound core biopsies. Among the 32 cases with MLL on core needle biopsy, 22 $(69 \%)$ were benign and $10(31 \%)$ had atypical ductal hyperplasia or detached groups of atypical cells. Of the 22 benign MLL, 19 (86\%) underwent excision: 15/19 (79\%) were benign, $3 / 19(16 \%)$ had atypical ductal hyperplasia and $1 / 19(5 \%)$ had ductal carcinoma in situ. Three of $22(14 \%)$ patients with benign MLL were observed (mean 28 months; range 10-60) and none developed carcinoma. Nine of $10(90 \%)$ patients with MLL with 
atypia underwent immediate excision: 5/9 (56\%) were benign, 1/9 (11\%) had atypical ductal hyperplasia and 3/9 (33\%) had invasive carcinoma (pure mucinous or mucinous features). Overall, a total of 4/32 (13\%) cases of MLL were upgraded to carcinoma, 3 of which were initially diagnosed on ultrasound core biopsies.

Conclusions: None of the patients in this series with benign MLL on core biopsy had invasive carcinoma on excision. However, 16\% had atypical ductal hyperplasia and $5 \%$ had ductal carcinoma in situ. Patients with MLL and atypical ductal hyperplasia or detached atypical cells on core biopsy were more likely to have invasive carcinoma on excision. These findings suggest that core biopsy findings may identify patients at the highest risk for upgrade to carcinoma on excision but validation in larger studies is required.

311 Expression Analysis of Epithelial-MesenchymalTransition Related Genes in Triple Negative Breast Cancer

Shuling Zhou, Anqi Li, Ming Li, Yan Xu, Yaoxing Xiao, Wentao Yang. Fudan University Shanghai Cancer Center, Shanghai, China.

Background: Triple negative breast cancer (TNBC) is a group of highly heterogeneous tumor. 6 subtypes were identified in TNBC, including basal-like 1, basal-like 2, immunomodulatory (IM), mesenchymal (M), mesenchymal stem-like (MSL), and luminal androgen receptor (LAR). M and MSL subtypes were closely related with epithelial mesenchymal transition (EMT).

Design: 179 cases of TNBC who underwent surgery in Fudan University Cancer Hospital were collected, including 145 cases of invasive carcinoma of no special type (ICONST), 12 cases of squamous cell carcinoma (SCC), 14 cases of spindle cell carcinoma (SpCC) and 8 cases of matrix producing carcinoma (MPC). Expression of EMT related genes (E-cadherin, slug, twist and vimentin) were dectected by immunohistochemistry in TNBC tissue array.

Results: 124 cases $(124 / 179,69.3 \%)$ were E-cadherin positive. Positive rate of E-cadherin in ICONST, SCC, SpCC and MPC patients were 78.6\% (114/145), $83.3 \%(10 / 12), 0.0 \%(0 / 14)$ and $0.0 \%(0 / 8)$. The expression was significantly higher in ICONST and SCC patients than in that of SpCC and MPC patients $(\mathrm{P}<0.0001) .51$ cases $(51 / 179,28.5 \%)$ were slug positive. Positive rate of slug in ICONST, SCC, SpCC and MPC patients were 19.4\%(28/145), 33.3\%(4/12), 78.6\%(11/14), 100.0\%(8/8) respectively. The expression of slug was significantly higher in SpCC and MPC patients than that in ICONST and SCC patients $(\mathrm{P}<0.05)$. 83 cases $(83 / 179,46.4 \%)$ were twist positive. Positive rate of slug in ICONST, SCC, SpCC and MPC patients were $19.4 \%(28 / 145), 33.3 \%(4 / 12), 78.6 \%(11 / 14), 100.0 \%(8 / 8)$, respectively. The expression was significantly higher in SpCC and MPC patients than that in ICONST and SCC patients $(\mathrm{P}<0.05) .67$ cases $(67 / 179,37.4 \%)$ were vimentin positive. Positive rate of vimentin in ICONST, SCC, SpCC and MPC patients were $30.3 \%(44 / 145), 8.3 \%(1 / 12)$, $100.0 \%(14 / 14), 100.0 \%(8 / 8)$. The expression was significantly higher in SpCC and MPC patients than that in ICONST and SCC patients $(\mathrm{P}<0.0001)$. The expression of Slug and Twist were negatively correlated with the expression of E-cadherin, while positively correlated with vimentin expression $(\mathrm{P}<0.05)$.

Conclusions: SpCC and MPC patients displayed lower expression of epithelial marker (E-cadherin), higher expression of EMT-related transcription factors (slug, twist) and mesenchymal marker (vimentin), which indicated the role of EMT in MPC and SpCC. Slug and twist may regulate the expression of vimentin and downregulate the expression of E-cadherin in TNBC.

312 Droplet Digital Polymerase Chain Reaction Detection of HER2 Amplification in Formalin Fixed Paraffin Embedded Breast and Gastric Carcinoma Samples

Ya-Zhen Zhu, Dan Lu, Maruja E Lira, Qing Xu, Yunzhi Du, Jianghong Xiong, Mao Mao, Hyun Cheol Chung, Guangjuan Zheng. Guangdong Provincial Hospital of Traditional Chinese Medicine (GPHTCM), Guangzhou, China; WuXi AppTec, Shanghai, China; Pfizer Oncology, San Diego, CA; Yonsei University College of Medicine, Seoul, Korea. Background: Human epidermal growth factor receptor 2 (HER2) is a key driver of tumorigenesis, and over-expression as a result of HER2 gene amplification has been observed in a number of solid tumors. Recently HER2 has become an important biomarker for the monoclonal antibody treatment of HER2-positive metastatic breast and advanced gastric cancer. The HER2 targeting antibody Trastuzumab treatment requires accurate measurement of HER2 levels for proper diagnosis. Droplet digital PCR (ddPCR) with highly direct, precise and absolute nucleic acid quantification could be used to detect HER2 amplification levels. The aim of the present study was to evaluate a robust, accurate and less subjective application of ddPCR for HER2 amplification levels and test the assay performance in clinical formalin-fixed paraffin-embedded (FFPE) breast and gastric carcinoma samples.

Design: Genomic DNA from HER2 amplified cell line SK-BR-3 was used to set up the ddPCR assays. The copy number of HER2 was compared to the chromosome 17 centromere reference gene (CEP17), expressed as HER2:CEP17 ratio. Genomic DNAs of FFPE specimens from 145 Asian patients with breast and gastric carcinomas were assayed using both standard methods, immunohistochemistry (IHC) and/or fluorescence in situ hybridization (FISH), and ddPCR.

Results: Based on 145 clinical breast and gastric carcinoma cases, our study demonstrated a high concordance of ddPCR results to FISH and IHC. In breast cancer specimens, the ddPCR results had high concordance with FISH and IHC defined HER2 status with a sensitivity of $90.9 \%$ (30/33) and a specificity of $100 \%$ (77/77). In gastric cancer specimens that were concordant in both FISH and IHC, our assay was $95.5 \%$ concordant with FISH and IHC (21/22).

Conclusions: ddPCR has the advantage of automation and also allows levels of HER2 amplification to be easily evaluated in large numbers of samples, and presents a potential option to define HER2 status.

\section{Cardiovascular Pathology}

313 Unusual Complications of Prosthetic Vascular Reconstruction: A Clinicopathologic Case Series

Ibrahim Aboshady, Naveed U Saqib, Deborah Vela, Ana M Segura, Kamal Khalil, L Maximilian Buja. Texas Heart Institute, Houston, TX; The University of Texas HSC, Houston, TX.

Background: Typical complications of vascular reconstructive surgery are thrombosis, infection pseudoaneurysm, dilation, and AE fistula/erosion. Certain, rarer complications are often ignored, unforeseen, or misinterpreted during surgery.

Design: We report 5 cases. Patient 1, a 72-year-old woman, died of an invasive intimal angiosarcoma (AS) detected in a 4.5-cm TAAA during surgical repair. Patients 2 and 3 had a superficial spreading intimal AS detected during replacement of Dacroncoated grafts implanted 5 and 9 years earlier. Patient 4 , a 61 -year-old woman who had undergone aortic repair, died of consequent acute severe bacterial meningitis involving the hippocampal region, choroid plexus, and pituitary gland. Patient 5 had a spinal cord infarct after undergoing open repair of inflammatory AAA.

Results: In the 3 AS cases, we studied - and sought therapeutic targets in - selective molecular pathways that may be involved in the transformation of benign to malignant endothelium. The proteins Akt/mTOR, STAT 3, COX-2, and CD44 were expressed in all lesions. H\&E staining showed thickened intima with calcified atherosclerotic lesions, and elastin staining showed diminished elastin fiber, medial degeneration, high-grade sarcoma, high N/C ratio, numerous atypical mitoses, and large areas of tumor necrosis. IHC results were strongly positive for vimentin, focally and unevenly positive for $C D$ 31 , and negative for pancytokeratin, CK-7, CK-20, calretinin, D-2-40, CD-45, S-100, MA, and CD 34; $95 \%$ of tumor cells were positive for Ki-67. The meningitis patient was anxious, tachypneic, hypothermic, paraplegic, and leukopenic $(3.1 \mathrm{k} / \mathrm{cmm})$ after aortic repair. CSF showed RBC 96,250, WBC 4100 (93\% PMN), glucose $<1$, protein 584, and visible gram-negative rods. MRI showed small left cerebellar and intraventricular hemorrhage; CSF, blood, and urine cultures grew Klebsiella pneumoniae.

Conclusions: These cases contribute to the body of knowledge regarding these unusual perioperative proximal and distal complications, whether immediate or delayed, helping reveal the spectrum of disease that may arise in vasculature tissues, either spontaneously or after synthetic-graft placement. To manage these unusual complications properly, surgeons should take timely and adequate biopsies from any ambiguous vascular reconstructive lesions for histopathologic study.

314 Regional Differences in Ascending Aortic Histology: A Comparative Histomorphologic Assessment with Implication for Pathologic Classification

Md Shahrier Amin, Cecilia Wu, Charles Leduc, Peter T Lin, Sarah M Jenkins, William D Edwards, Joseph J Maleszewski. Mayo Clinic, Rochester, MN.

Background: The aortic sinuses (AS) are often involved in cases of aneurysm and/ or dissection. In contrast to the tubular aorta (TA), the histomorphologic features of the AS have yet to be systematically characterized, which can potentially lead to diagnostic misinterpretation. Accurate knowledge of regional variations in aortic histomorphology is also useful in the design of prosthetic biomaterials. The aim of this study was to determine the range of histomorphologic features, in the normal AS across a broad age range.

Design: Autopsy-derived ascending aortic specimens were prospectively collected over a 1-year period. Decedents with a history of significant cardiovascular disease were excluded. Transverse sections of the AS and TA were collected and stained with H\&E and VVG. The following parameters were blindly evaluated by 3 pathologists: medial thickness, elastic tissue amount, lamellar units per 200X field, pattern of major elastic fibers (parallel or woven) and intralamellar distance. Measures were compared between AS and TA with paired t-test. Linear regression was used for comparisons with age. Results: 32 cases (17 women, mean age 56.9 years, range 12-85; 15 men, mean age 62 years, range 13-90) met inclusion criteria. The media was significantly thicker in the TA $(1215 \pm 213 \mathrm{~mm})$ vs. the AS $(879 \pm 249 \mathrm{~mm})(\mathrm{p}<0.0001)$. While there was no difference in the quantity of elastin, there were significantly more lamellar units in the TA $(29 \pm 5)$ vs AS $(25 \pm 5)(\mathrm{p}<0.0001)$ with smaller intralamellar distance (TA $21.4 \pm 4.2$ $\mathrm{mm}$ vs AS $23.4 \pm 4.6 \mathrm{~mm})(\mathrm{p}=0.0006)$. A woven pattern of elastic fibers was more commonly identified in the AS $(93.8 \%)$, while a parallel pattern was more commonly identified in the TA $(90.6 \%)(\mathrm{p}<0.0001)$. Ratio of media thickness between AS to TA decreased with age $(\mathrm{p}=0.04)$, as did the distance between elastic fibers in the AS only $(\mathrm{p}=0.003)$ and not in the TA.

Conclusions: This is the first study to systematically and comparatively evaluate the histomorphology of the AS across a broad age range. The results highlight the unique histomorphometric features of the AS, that can mimic medial degenerative changes and lead to mischaracterization. This underscores the importance of proper orientation and sampling of surgically resected aortas. Examination for region-specific pathologies in various disease states is ongoing. While the functional significance is not yet clear, the decrease in AS intralamellar elastic fiber distance with age might contribute to the age-associated increase in aortic root diameter. 